\title{
MANUAL
}

INL/EXT-18-44465

Revision 0

Printed June 2018

\section{RAVEN User Guide}

Andrea Alfonsi, Cristian Rabiti, Diego Mandelli, Joshua Cogliati, Congjian Wang, Paul W. Talbot, Daniel P. Maljovec

Prepared by

Idaho National Laboratory

Idaho Falls, Idaho 83415

The Idaho National Laboratory is a multiprogram laboratory operated by

Battelle Energy Alliance for the United States Department of Energy

under DOE Idaho Operations Office. Contract DE-AC07-05ID14517.

Approved for unlimited release.

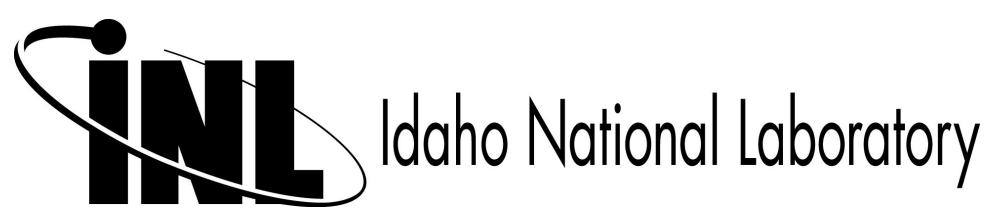


Issued by the Idaho National Laboratory, operated for the United States Department of Energy by Battelle Energy Alliance.

NOTICE: This report was prepared as an account of work sponsored by an agency of the United States Government. Neither the United States Government, nor any agency thereof, nor any of their employees, nor any of their contractors, subcontractors, or their employees, make any warranty, express or implied, or assume any legal liability or responsibility for the accuracy, completeness, or usefulness of any information, apparatus, product, or process disclosed, or represent that its use would not infringe privately owned rights. Reference herein to any specific commercial product, process, or service by trade name, trademark, manufacturer, or otherwise, does not necessarily constitute or imply its endorsement, recommendation, or favoring by the United States Government, any agency thereof, or any of their contractors or subcontractors. The views and opinions expressed herein do not necessarily state or reflect those of the United States Government, any agency thereof, or any of their contractors.

Printed in the United States of America. This report has been reproduced directly from the best available copy.

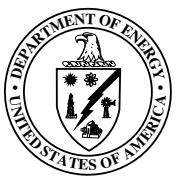


INL/EXT-18-44465

Revision 0

Printed June 2018

\title{
RAVEN User Guide
}

\author{
Andrea Alfonsi \\ Cristian Rabiti \\ Diego Mandelli \\ Joshua Cogliati \\ Congjian Wang \\ Paul W. Talbot \\ Daniel P. Maljovec
}




\section{Contents}

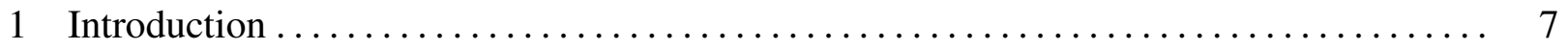

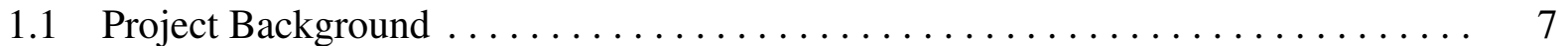

1.2 Acquiring and Installing RAVEN $\ldots \ldots \ldots \ldots \ldots \ldots \ldots \ldots \ldots \ldots \ldots \ldots \ldots \ldots \ldots \ldots \ldots \ldots \ldots$

1.3 User Guide Formats . . . . . . . . . . . . . . . . . . . . . . . . . . 8

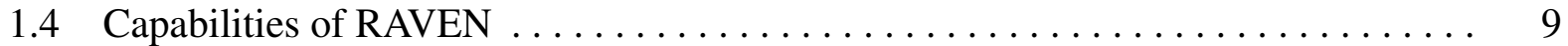

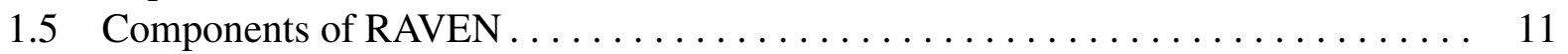

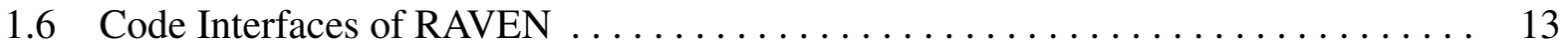

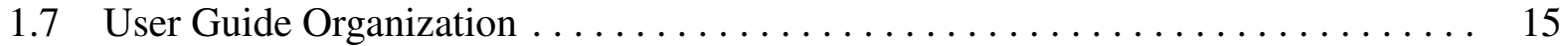

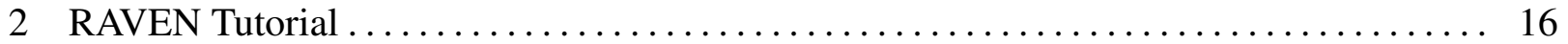

2.1 Example Model: Analytic Bateman ........................ 16

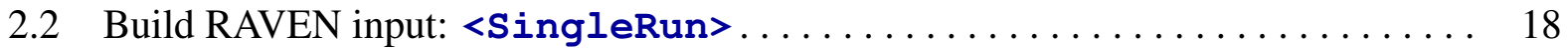

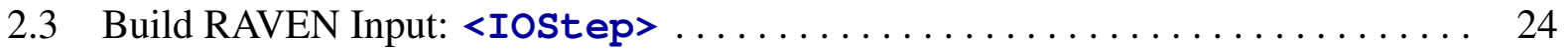

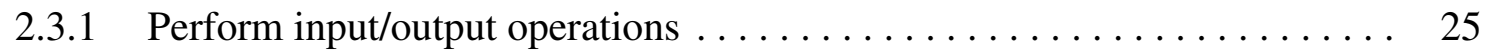

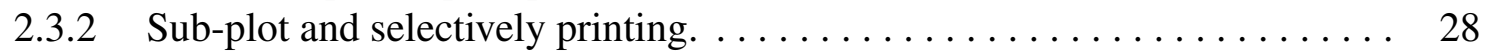

2.4 Build RAVEN Input: <MultiRun> $\ldots \ldots \ldots \ldots \ldots \ldots \ldots \ldots \ldots \ldots \ldots \ldots \ldots \ldots \ldots \ldots$

2.5 Build RAVEN Input: <RomTrainer $\ldots \ldots \ldots \ldots \ldots \ldots \ldots \ldots \ldots \ldots \ldots . \ldots \ldots$

2.5.1 How to train and output a ROM? .................... 41

2.5.2 How to load and sample a ROM? .................... 45

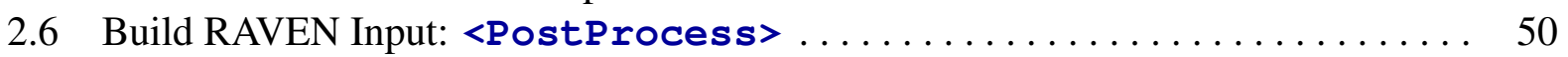

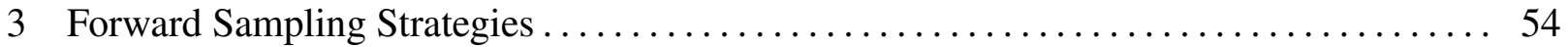

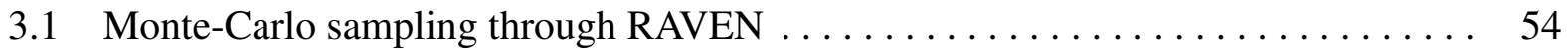

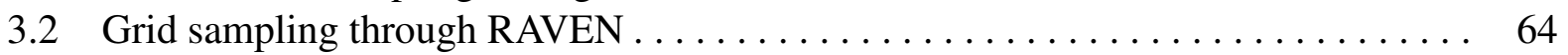

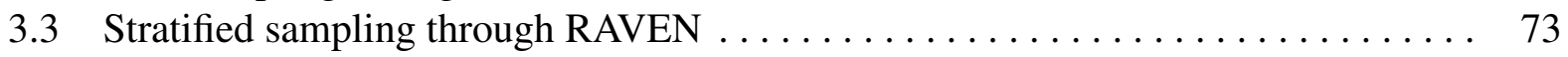

3.4 Sparse Grid Collocation sampling through RAVEN $\ldots \ldots \ldots \ldots \ldots \ldots \ldots \ldots . \ldots 3$

4 Adaptive Sampling Strategies ..................................... 99

4.1 Limit Surface Search sampling through RAVEN $\ldots \ldots \ldots \ldots \ldots \ldots \ldots \ldots \ldots$

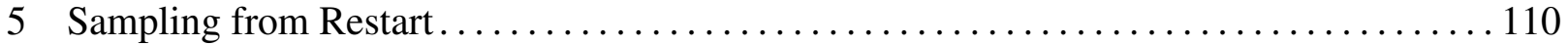

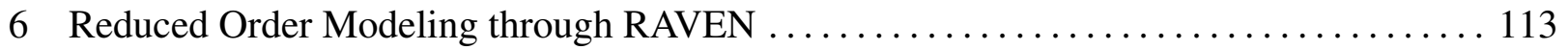

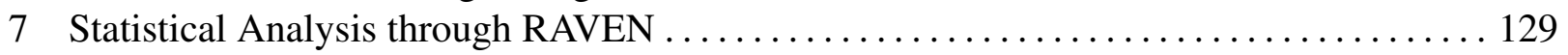

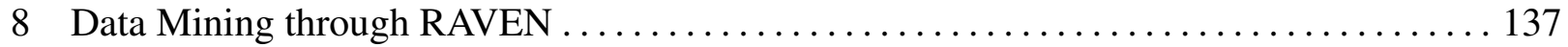

9 Model Optimization .............................................. 148

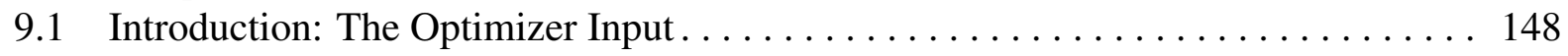

$9.1 .1 \quad$ Models ..................................... 150

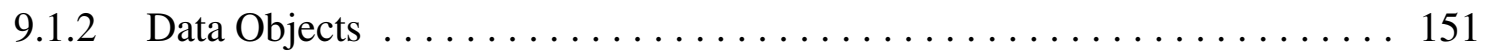

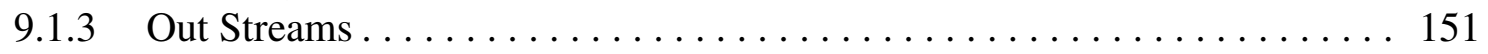

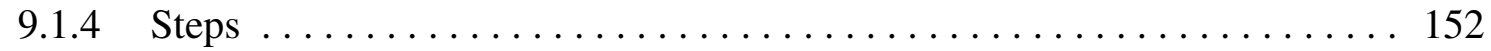

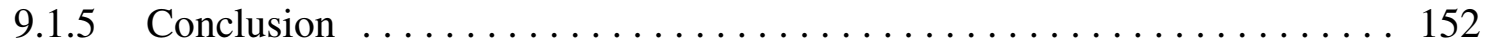

9.2 Initial Conditions and Parallel Trajectories $\ldots \ldots \ldots \ldots \ldots \ldots \ldots \ldots \ldots \ldots \ldots \ldots \ldots \ldots$

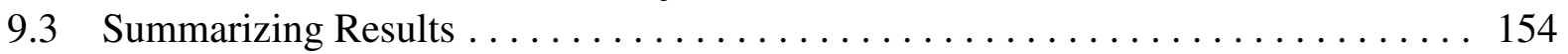

9.4 Adjusting Adaptive Steps .................................. 155 


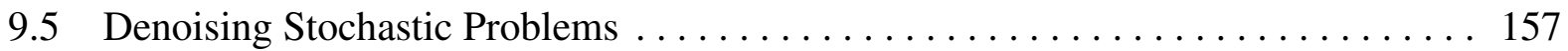

9.6 Input Space Subdivision . . . . . . . . . . . . . . . . . . . . . . . . . . 159

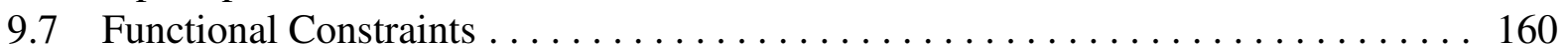

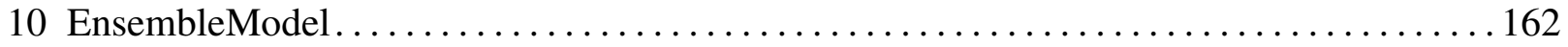

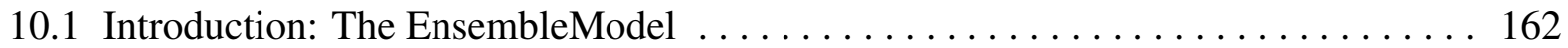

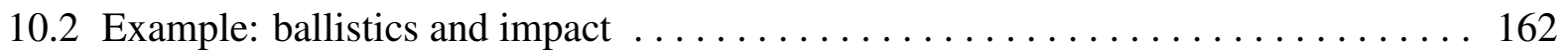

10.2.1 DataObjects ..................................... 163

10.2 .2 Files ......................................... 164

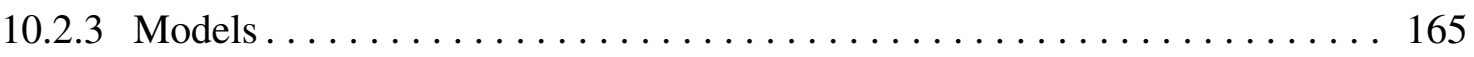

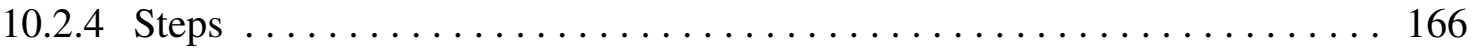

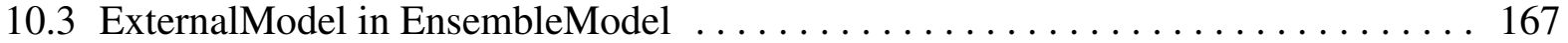

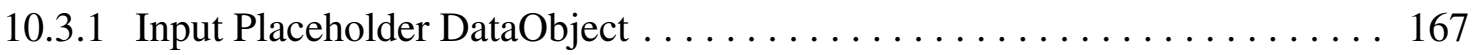

10.3 .2 DataSet . . . . . . . . . . . . . . . . . . . . . . . . . 168

10.3.3 Scalar Variables .................................. 168

10.3.4 Independent Variables . . . . . . . . . . . . . . . . . . . . . . . . . . . 169

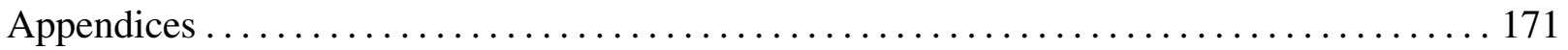

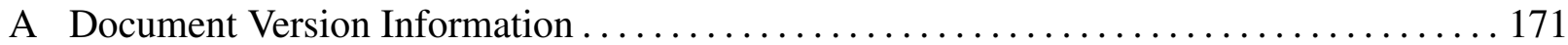

References...................................................... 172 


\section{Introduction}

\subsection{Project Background}

The development of RAVEN started in 2012 when, within the Nuclear Energy Advanced Modeling and Simulation (NEAMS) program [1], the need of a modern risk evaluation framework arose. RAVEN's principal assignment is to provide the necessary software and algorithms in order to employ the concepts developed by the Risk Informed Safety Margin Characterization (RISMC) Pathway. RISMC is one of the pathways defined within the Light Water Reactor Sustainability (LWRS) program [2].

The goal of the RISMC approach is the identification not only of the frequency of an event which can potentially lead to system failure, but also the proximity (or lack thereof) to key safetyrelated events: the safety margin. Hence, the approach is interested in identifying and increasing the safety margins related to those events. A safety margin is a numerical value quantifying the probability that a safety metric (e.g. peak pressure in a pipe) is exceeded under certain conditions. Most of the capabilities, implemented having Reactor Excursion and Leak Analysis Program v.7 (RELAP-7) as a principal focus, are easily deployable to other system codes. For this reason, several side activates have been employed (e.g. RELAP5-3D [3], any Multiphysics Object Oriented Simulation Environment-based App, etc.) or are currently ongoing for coupling RAVEN with several different software.

\subsection{Acquiring and Installing RAVEN}

RAVEN is supported on three separate computing platforms: Linux, OSX (Apple Macintosh), and Microsoft Windows. Currently, RAVEN is open-source and downloadable from RAVEN GitHub repository: https://github.com/idaholab/raven. New users should visit https: //github.com/idaholab/raven/wiki or refer to the user manual [4] to get started with RAVEN. This typically involves the following steps:

- Download RAVEN

You can download the source code of RAVEN from https : / / github . com/idaholab/ raven.

- Install RAVEN dependencies

Instructions are available from https://github.com/idaholab/raven/wiki, or the user manual [4].

- Install RAVEN

Instructions are available from https://github.com/idaholab/raven/wiki, or the user manual [4]. 
- Run RAVEN

If RAVEN is installed successfully, please run the regression tests to verify your installation:

. Irun_tests

Normally there are skipped tests because either some of the codes are not available, or some of the test are not currently working. The output will explain why each is skipped. If all the tests pass, you are ready to run RAVEN. Now, open a terminal and use the following command (replace <inputFileName. xml> with your RAVEN input file):

raven_framework <inputFileName.xml>

where the raven framework script can be found in the RAVEN folder. Alternatively, the Driver.py script contained in the folder "raven/framework" can be directly used:

python raven/framework/Driver.py <inputFileName.xml>

- Participate in RAVEN user communities

Join RAVEN mail lists to get help and updates of RAVEN: https : / / groups . google. com/forum/\#! forum/inl-raven-users.

\subsection{User Guide Formats}

In order to highlight some parts of the user guide having a particular meaning (input structure, examples, terminal commands, etc.), specific formats have been used. This section provides the formats with a specific meaning:

- Python Coding:

class AClass ():

def aMethodImplementation(self):

pass

- RAVEN XML input example:

$<$ MainXMLBlock $>$

- .

<aXMLnode name='anObjectName' anAttribute='aValue' >

$<$ aSubNode $>$ body $</$ aSubNode $>$

$</$ aXMLnode $>$

$<$ !-- This is commented block -->

...

$</$ MainXMLBlock $>$ 
- Bash Commands:

cd trunk/raven/

. Iraven_libs_script.sh

cd ... . . /

\subsection{Capabilities of RAVEN}

RAVEN [5] [6] [7] [8] is a software framework that allows the user to perform parametric and stochastic analysis based on the response of complex system codes. The initial development was designed to provide dynamic probabilistic risk analysis capabilities (DPRA) to the thermalhydraulic code RELAP-7 [9], currently under development at Idaho National Laboratory (INL). Now, RAVEN is not only a framework to perform DPRA but it is a flexible and multi-purpose uncertainty quantification, regression analysis, probabilistic risk assessment, data analysis and model optimization platform. Depending on the tasks to be accomplished and on the probabilistic characterization of the problem, RAVEN perturbs (e.g., Monte-Carlo, Latin hypercube, reliability surface search) the response of the system under consideration by altering its own parameters. The system is modeled by third party software (e.g., RELAP5-3D, MAAP5, BISON, etc.) and accessible to RAVEN either directly (software coupling) or indirectly (via input/output files). The data generated by the sampling process is analyzed using classical statistical and more advanced data mining approaches. RAVEN also manages the parallel dispatching (i.e. both on desktop/workstation and large High Performance Computing machines) of the software representing the physical model. RAVEN heavily relies on artificial intelligence algorithms to construct surrogate models of complex physical systems in order to perform uncertainty quantification, reliability analysis (limit state surface) and parametric studies.

The main capabilities of RAVEN, with brief descriptions, are summarized here, or one can check the Figure. 1. These capabilities may be used on their own or as building blocks to construct the sought workflow. In addition, RAVEN also provides some more sophisticated Ensemble algorithms such as EnsembleForward, EnsembleModel to combine the existing capabilites.

- Sensitivity Analysis and Uncertainty Quantification: Sensitivity analysis is a mathematical tool that can be used to identify the key sources of uncertainties. Uncertainty quantification is a process by which probabilistic information about system responses can be computed according to specified input parameter probability distributions. Available approaches in RAVEN include Monte Carlo, Grid, Stratified (Latin hypercube), Sparse Grid Collocation, Sobol, Adaptive Sparse Grid, Adaptive Sobol and BasicStatistics.

- Design of Experiments: The design of experiments (DOE) is a powerful tool that can be used to explore the parameter space at a variety of experimental situations. It can be used 


\begin{tabular}{|l|l|l|l|l|l|l|}
\hline \multirow{2}{*}{ Algorithms } & \multicolumn{5}{|c|}{ User Cases } \\
\cline { 2 - 7 } & $\begin{array}{l}\text { Uncertainty } \\
\text { Quantification }\end{array}$ & $\begin{array}{l}\text { Risk } \\
\text { Analysis }\end{array}$ & $\begin{array}{l}\text { Risk } \\
\text { Management }\end{array}$ & Validation & $\begin{array}{l}\text { Experiment } \\
\text { design }\end{array}$ & Optimization \\
\hline Distribution & Yes & Yes & Yes & Yes & Yes & Yes \\
\hline Sampling & Yes & Yes & Yes & Yes & Yes & Yes \\
\hline Surrogate Models & Yes & Yes & Yes & Yes & Yes & Yes \\
\hline $\begin{array}{l}\text { Statistical post } \\
\text { processing }\end{array}$ & Yes & Yes & Yes & Yes & Yes & Yes \\
\hline Reliability Surface & & Yes & Yes & & & \\
\hline Data mining & & Yes & Yes & & & \\
\hline $\begin{array}{l}\text { Analytical distribution } \\
\text { comparison (ongoing) }\end{array}$ & & & & Yes & Yes & \\
\hline $\begin{array}{l}\text { Minimization } \\
\text { Algorithms (ongoing) }\end{array}$ & & & Yes & & Yes & Yes \\
\hline
\end{tabular}

Figure 1. RAVEN Capabilities vs. Needs

to determine the relationship between input factors and the desired outputs. Available approaches in RAVEN include Factorial Design (i.e. General full factorial, 2-level fractionalfactorial and Plackett-Burman) and Response Surface Design (i.e. Box-Behnken and Central composite algorithms).

- Risk Mitigation or Model Optimization: RAVEN uses the Optimizer, a powerful samplerlike entity that searches the input space to find minimum or maximum values of a reponse. Currently available optimizers include Simultaneous Perturbation Stochastic Approximation (SPSA).

- Risk Analysis: Available approaches in RAVEN include Dynamic Event Tree, Limit Surface Search, Hybrid Dynamic Event Tree, Adaptive Dynamic Event Tree, Adaptive Hybrid Dynamic Event Tree, Data Mining, Importance Rank, Safest Point, and Basic Statistics.

- Risk Management: Available approaches in RAVEN include Reduced order models, approaches used for sensitivity and uncertainty analysis, and Dynamic Event Tree methods.

- Validation: Available approaches in RAVEN include ROMs, Comparison Statistics and Validation Metrics 
In addition, RAVEN includes a number of related advanced capabilities. Surrogate or Reduced order models (ROMs) are mathematical model trained to predict a response of interest of a physical system. Typically, ROMs trade speed for accuracy representing a faster, rough estimate of the underlying systems. They can be used to explore the input parameter space for optimization or sensitivity and uncertainty studies. Ensemble Model is able to combine Codes, External Models and ROMs. It is intended to create a chain of models whose execution order is determined by the input/output relationships among them. If the relationships among the models evolve in a non-linear system, a Picard's iteration scheme is employed.

\subsection{Components of RAVEN}

The RAVEN code does not have a fixed calculation flow, since all of its basic objects can be combined in order to create a user-defined calculation flow. Thus, its input, eXtensible Markup Language (XML) format, is organized in different XML blocks, each with a different functionality. For more information about XML, please click on the link: XML tutorial.

The main input blocks are as follows:

- <Simulation>: The root node containing the entire input, all of the following blocks fit inside the Simulation block.

- <RunInfo>: Specifies the calculation settings (number of parallel simulations, etc.).

- <Files>: Specifies the files to be used in the calculation.

- <Distributions>: Defines distributions needed for describing parameters, etc.

- <Samplers>: Sets up the strategies used for exploring an uncertain domain.

- <DataObjects>: Specifies internal data objects used by RAVEN.

- <Databases>: Lists the HDF5 databases used as input/output to a RAVEN run.

- <OutStreams>: Visualization and Printing system block.

- <Models>: Specifies codes, ROMs, post-processing analysis, etc.

- <Functions>: Details interfaces to external user-defined functions and modules the user will be building and/or running.

- <VariableGroups>: Creates a collection of variables.

- <Optimizers>: Performs the driving of a specific goal function over the model for value optimization.

- <Metrics>: Calculate the distance values among points and histories. 
- <Steps>: Combines other blocks to detail a step in the RAVEN workflow including I/O and computations to be performed.

Each of these components are explained in dedicated sections of the user manual [4], and can be used as building blocks to construct certain calculation flow, as shown in Figure. 2 . In this guide, we will only show how to use these components to build the analysis flow, and we recommend the user to check the user manual [4] for the detailed descriptions.

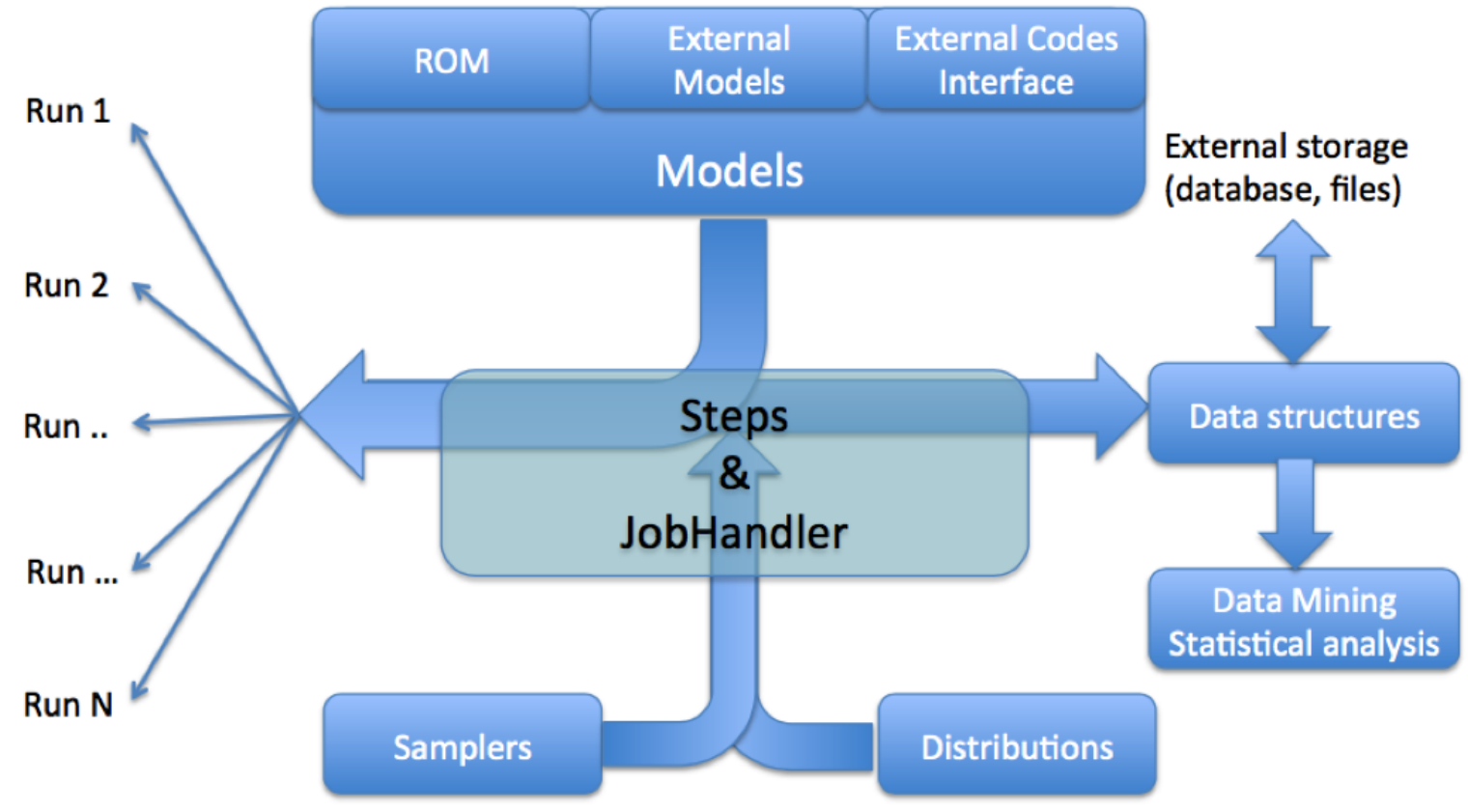

Figure 2. RAVEN structures

In addition, RAVEN allows the user to load any external input file that contains the required XML nodes into the RAVEN main input file, and provide the standard XML comments, using $<$ ! -and $-->$. For example, one can use the following template to load the $<$ Distributions $>$ from file 'Distributions.xml'.

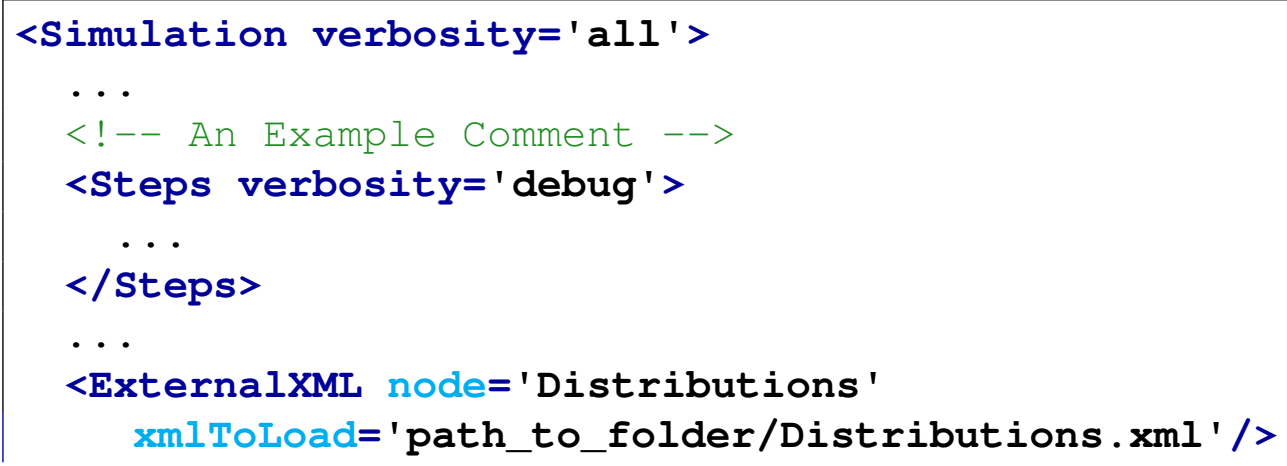


RAVEN also allows the user to control the level of output to the user interface by using verbosity system. These settings can be declared globally as attributes in the <Simulation $>$ node, or locally in each block node as shown in above template. The verbosity levels are

- 'silent' - Only simulation-breaking errors are displayed.

- ' quiet' - Errors as well as warnings are displayed.

- 'all' (default) - Errors, warnings, and messages are displayed.

- ' debug' - For developers. All errors, warnings, messages, and debug messages are displayed.

\subsection{Code Interfaces of RAVEN}

The procedure of coupling a new code/application with RAVEN is a straightforward process. The provided Application Programming Interfaces (APIs) allow RAVEN to interact with any code as long as all the parameters that need to be perturbed are accessible by input files or via python interfaces. For example, for all the codes currently supported by RAVEN (e.g. RELAP-7, RELAP-5D, BISON, MAMMOTH, etc.), the coupling is performed through a Python interface that interprets the information coming from RAVEN and translates them into the input of the driven code. The couping procedure does not require modifying RAVEN itself. Instread, the developer creates a new Python interface that is going to be embedded in RAVEN at run-time (no need to introduce hard-coded coupling statements). In addition, RAVEN will manage concurrent executions of your simulations in parallel, whether on a local desktop or remote high-performance cluster.

Figure. 3 depicts the different APIs between RAVEN and the computational models, i.e. the ROM, External Models and External Code APIs. 


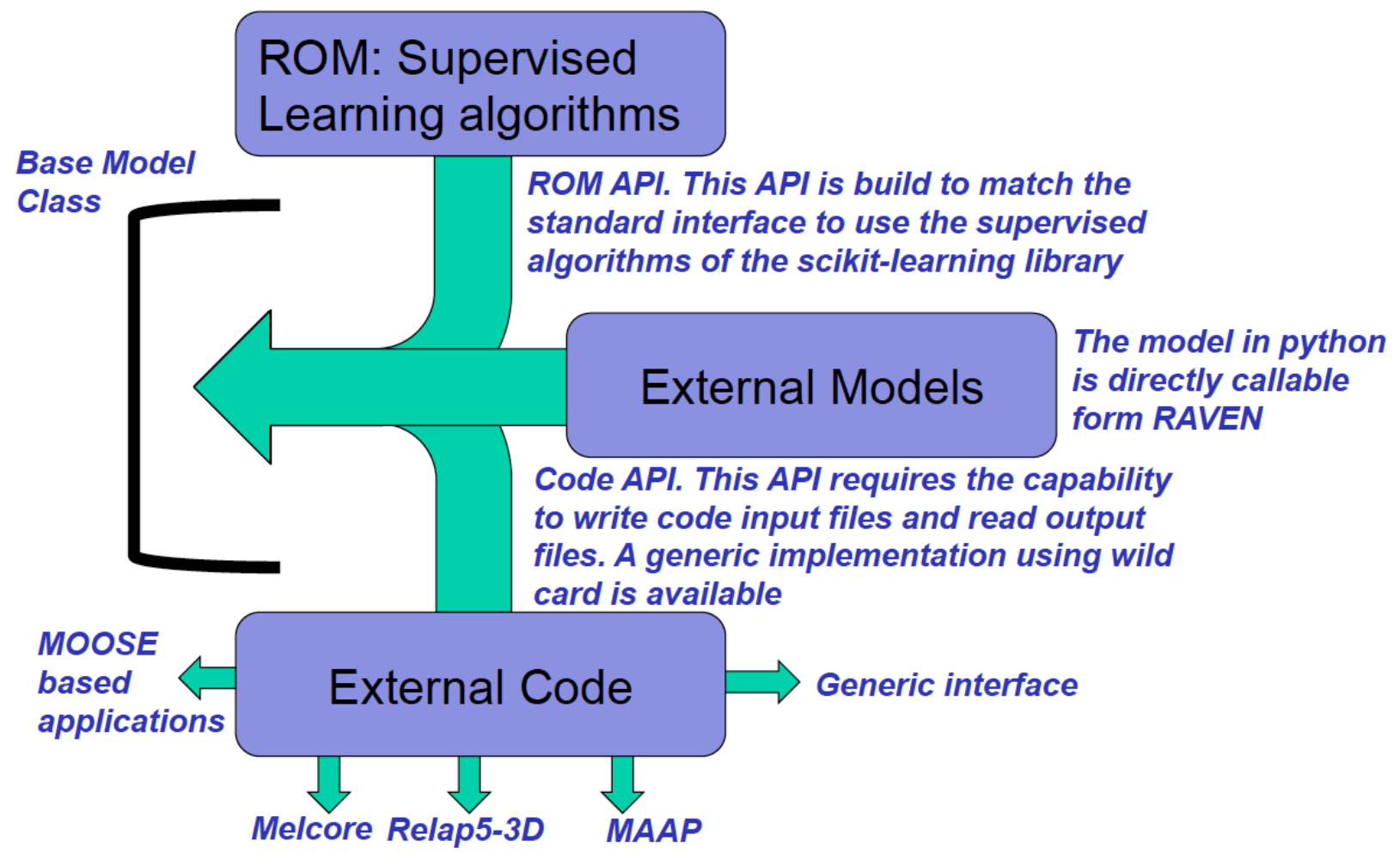

Figure 3. RAVEN Application Programming Interfaces 


\subsection{User Guide Organization}

The goal of this document is to provide a set of detailed examples that can help the user to become familiar with the RAVEN code. RAVEN is capable of investigating system response and explore input space using various sampling schemes such as Monte Carlo, grid, or Latin Hypercube. However, RAVEN strength lies in its system feature discovery capabilities such as: constructing limit surfaces, separating regions of the input space leading to system failure, and using dynamic supervised learning techniques. New users should consult the RAVEN Tutorial to get started.

- RAVEN Tutorial: section 2

- Sampling Strategies: section 3 and section 4

- Restart: section 5

- Reduced Order Modeling: section 6

- Risk Analysis: section 7

- Data Mining: section 8

- Model Optimization: section 9 


\section{RAVEN Tutorial}

\subsection{Example Model: Analytic Bateman}

This section is intended for the new users to familiarize them with how to perform their studies through RAVEN. A simple example, conventionally called AnalyticBateman, has been developed. It solves a system of ordinary differential equations (ODEs), of the form:

$$
\left\{\begin{array}{l}
\frac{\mathrm{d} \mathbf{X}}{\mathrm{d} t}=\mathbf{S}-\mathbf{L} \\
\mathbf{X}(t=0)=\mathbf{X}_{\mathbf{0}}
\end{array}\right.
$$

where:

- $\mathrm{X}_{\mathbf{0}}$, initial conditions

- S, source terms

- $\mathbf{L}$, loss terms

For example, this code is able to solve a system of two ODEs as follows:

$$
\left\{\begin{array}{l}
\frac{\mathrm{d} x_{1}}{\mathrm{~d} t}=\phi(t) \times \sigma_{x_{1}}-\lambda_{x_{1}} \times x_{1}(t) \\
\frac{\mathrm{d} x_{2}}{\mathrm{~d} t}=\phi(t) \times \sigma_{x_{2}}-\lambda_{x_{2}} \times x_{2}(t)+x_{1}(t) \times \lambda_{x_{1}} \\
x_{1}(t=0)=x_{1}^{0} \\
x_{2}(t=0)=0.0
\end{array}\right.
$$

The input of the AnalyticBateman code is in XML format. For example, the following is the reference input for a system of 4 Ordinary Differential Equations (ODEs) that is going to be used for as an example in this guide. All the files required for this system are located at "raven/tests/framework/user_guide/physicalCode".

raven/tests/framework/user_guide/physicalCode/analyticalbateman/Input.xml

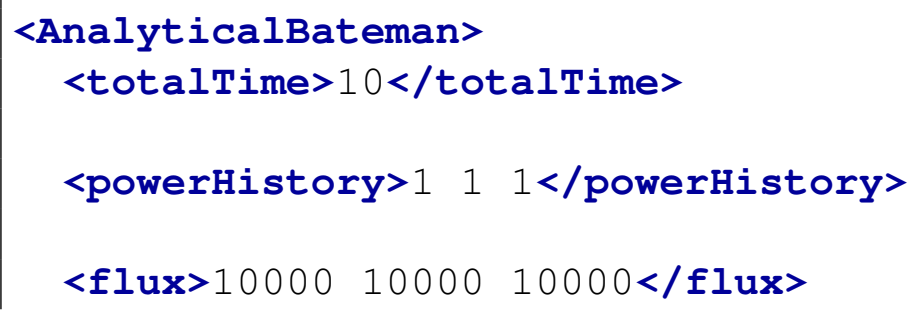




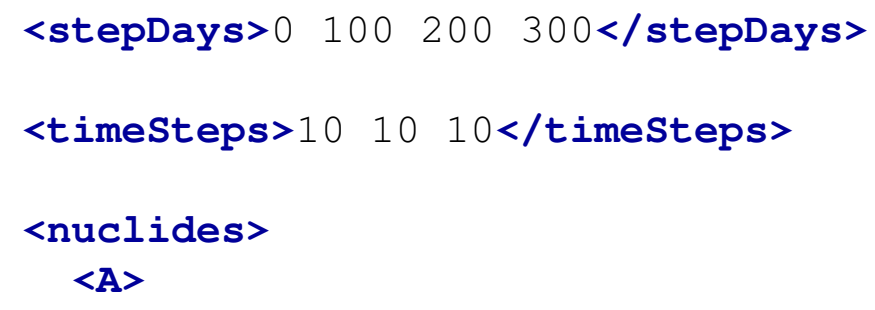

The code outputs the time evolution of the 4 variables $(A, B, C, D)$ in a CSV file, producing the following output: 
Table 1. Reference case sample results.

$\begin{array}{lllll}\text { time } & \text { A } & \text { C } & \text { B } & \text { D } \\ 0 & 1.0 & 1.0 & 1.0 & 1.0 \\ 2880000.0 & 0.983434738239 & 0.977851848235 & 1.01011506729 & 1.01013172275 \\ 5760000.0 & 0.967143884376 & 0.956202457404 & 1.01936231677 & 1.02036100400 \\ 8640000.0 & 0.951122892771 & 0.935040450532 & 1.02777406275 & 1.03067925987 \\ 10368000.0 & 0.941637968936 & 0.922572556179 & 1.03243314106 & 1.03690947068 \\ 12096000.0 & 0.932247632016 & 0.910273757371 & 1.03680933440 & 1.04316700086 \\ 13824000.0 & 0.922950938758 & 0.898141730426 & 1.04090912054 & 1.04945015916 \\ 15552000.0 & 0.913746955315 & 0.886174183908 & 1.04473885709 & 1.05575729317 \\ 17280000.0 & 0.904634757153 & 0.874368858183 & 1.04830478357 & 1.06208678854 \\ 20736000.0 & 0.886682064542 & 0.851235986899 & 1.05466958557 & 1.07480659230 \\ 24192000.0 & 0.869085647400 & 0.828725658721 & 1.06005115510 & 1.08759739100 \\ 27648000.0 & 0.851838435355 & 0.806820896763 & 1.06449535534 & 1.10044757060 \\ 31104000.0 & 0.834933498348 & 0.785505191756 & 1.06804634347 & 1.11334606143 \\ 34560000.0 & 0.818364043850 & 0.764762489077 & 1.07074662835 & 1.12628231792\end{array}$

\subsection{Build RAVEN input: <SingleRun>}

In this section, we will show the user how to use RAVEN to run a single instance of a driven code, and printing some variables. We will start to build a very simple RAVEN input, and this input file can be found at "raven/tests/framework/user_guide/ravenTutorial/singleRun.xml". From this process, we hope the user can get a better idea about RAVEN entities and learn how to build their own RAVEN inputs for their applications. In order to accomplish these tasks, the following procedures are needed:

\section{Set up the running environment: <RunInfo>}

The RunInfo entity is an information container which describes how the overall computation should be performed. This Entity accepts several input settings that define how to drive the calculation and set up, when needed, particular settings for the machine the code needs to run on (queue system, if not Portable Batch System-PBS, etc.). For the simple case, the RunInfo will look like:

raven/tests/framework/user_guide/ravenTutorial/singleRun.xml

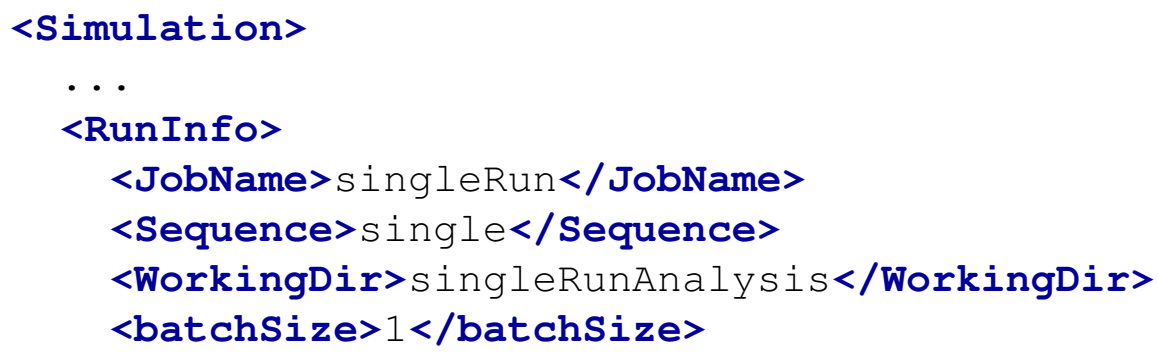


In this specific case, only one step named ' single' is going to be sequentially run using a single processor as defined by <Batchsize>. All the output files and temporary files will be dumped in the folder 'singleRunAnalysis'.

\section{Provide the required files: $\langle$ Files $>$}

The Files entity defines any files that might be needed within the RAVEN run. This could include inputs to the Model, pickled ROM files, or Comma Separated Value (CSV) files for post-processors, to name a few. Each entry in the $\langle$ Files $>$ block is a tag with the file type. Files given through the input XML at this point are all <Input> type. Each <Input> node has a required attributes name. It does not need to be the actual filename, and it is the name by which RAVEN will use to identify the specific file. Other optional attributes are not directly used by RAVEN, and they are mainly used by the CodeInterface. More detailed information can be found in the user manual [4]. For the simple case, the Files will look like:

raven/tests/framework/user_guide/ravenTutorial/singleRun.xml

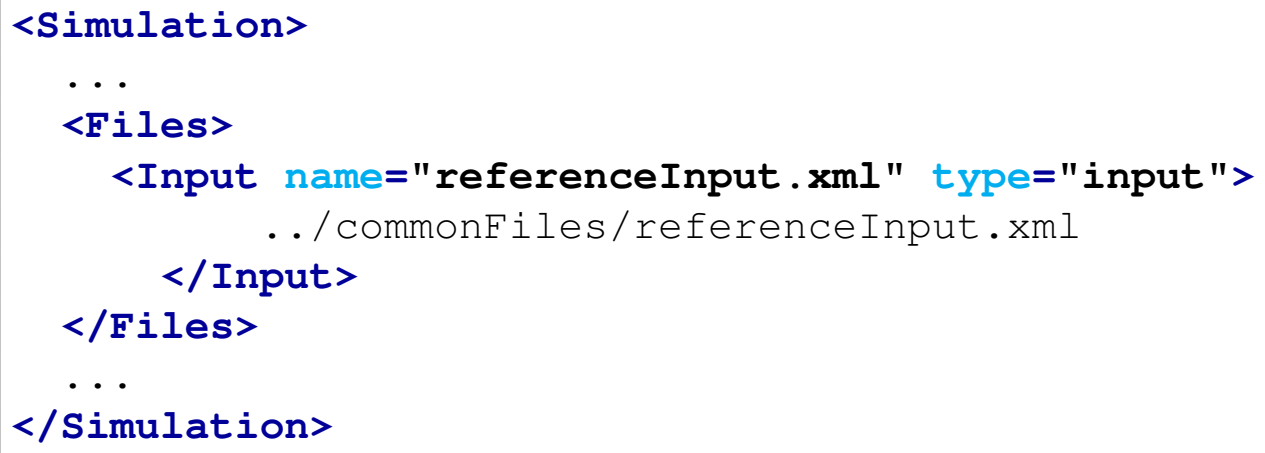

This RAVEN input file shows that the user will provide a file that is located at "../commonFiles/referenceInput.xml" with reference name ' referenceInput. xml'. This file will be available for use via other RAVEN input blocks or entities. In this case, a relative path to the working directory specified via <WorkingDir $>$ under node $<$ RunInfo $>$ is used.

\section{Link between RAVEN and driven code: <Models>}

The Models entity represents the projection from the input to the output space. In other words, the Model entity can be seen as a transfer function between the input and output space. Currently, RAVEN defines the following sub-entities:

- Code, represents the driven code, through external code interfaces (see [4])

- ExternalModel, represents a physical or mathematical model that is directly implemented by the user in a Python module 
- $R O M$, represents the Reduced Order Model, interfaced with several algorithms

- HybridModel, automatic/smart Entity to automatically choose between a ROM (or a set of them) and an High-Fidelity Model (e.g. ExternalModel, Code)

- PostProcessor, is used to perform action on data, such as computation of statistical moments, correlation matrices, etc.

For simplicity, only Code is used here for the demonstration, and the input block looks like:

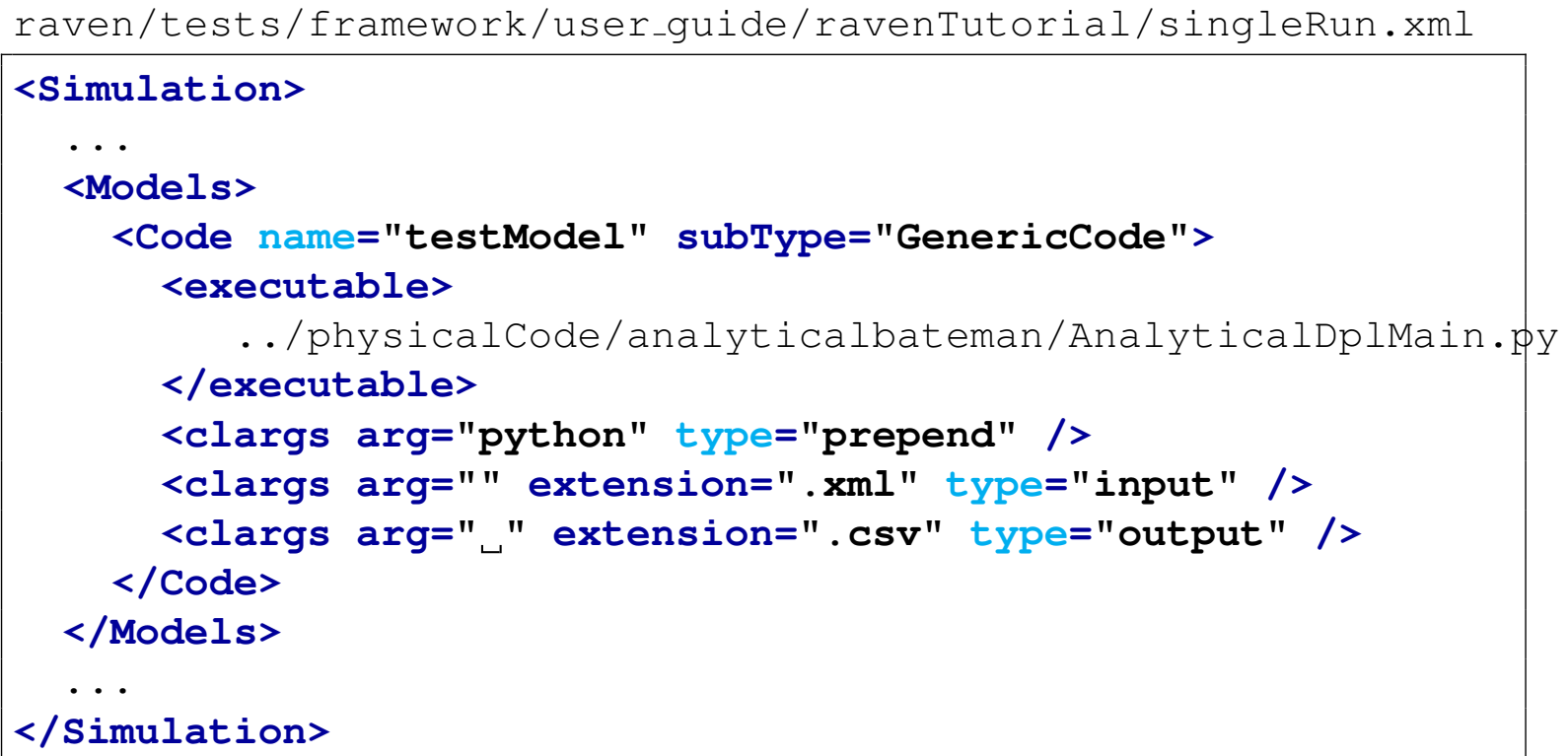

As shown in the <Models $>$ block, the subnodes defined for $<$ Code $>$ is equivalent to: python .. /physicalcode/analyticalbateman/AnalyticaldplMain.py

with the requirement of extensions of input and output files, as defined via 〈clargs $\rangle$, to be ' . $\mathbf{x m l} \mathbf{l}^{\prime}$ and ' . $\mathbf{c s v}$ ', respectively. In this case, the GenericCode interface is employed. This interface is meant to handle a wide variety of generic codes that take straightforward input files and produce CSV files. Note: If a code contains cross-dependent data, the generic interface is not applicable. For more detailed information, the user can refer to section Existing Interface of the user manual [4].

\section{Container of input and output data: <DataObjects>}

The DataObjects system is a container of data objects of various types that can be constructed during the execution of desired calculation flow. These data objects can be used as input or output for a particular Model Entity. Currently RAVEN supports the following data types, each with a particular conceptual meaning:

- PointSet is a collection of individual objects, each describing the state of the system at a certain point (e.g. in time). It can be considered a mapping between multiple sets of parameters in the input space and the resulting sets of outcomes in the output space at a particular point (e.g., in time). 
- HistorySet is a collection of individual objects, each describing the temporal evolution of the state of the system within a certain input domain. It can be considered a mapping between multiple sets of parameters in the input space and the resulting sets of temporal evolution in the output space.

- DataSet is a collection of individual objects and a generalization of the previously described DataObjects, aimed to contain a mixture of data (scalars, arrays, etc.). The variables here stored can be independent (i.e. scalars) or dependent (arrays) on certain dimensions (e.g. time, coordinates, etc.). It can be considered a mapping between multiple sets of parameters in the input space (both dependent and/or independent) and the resulting sets of evolution in the output space (bothdependentand/orindependent).

The DataObjects represent the designeted way to transfer the information coming from a Model (e.g., the driven code) to all the other RAVEN systems (e.g., Out-Stream system, Reduced Order Modeling component, etc.). For the simple case, the <DataObjects> block of RAVEN input is:

raven/tests/framework/user_guide/ravenTutorial/singleRun.xml

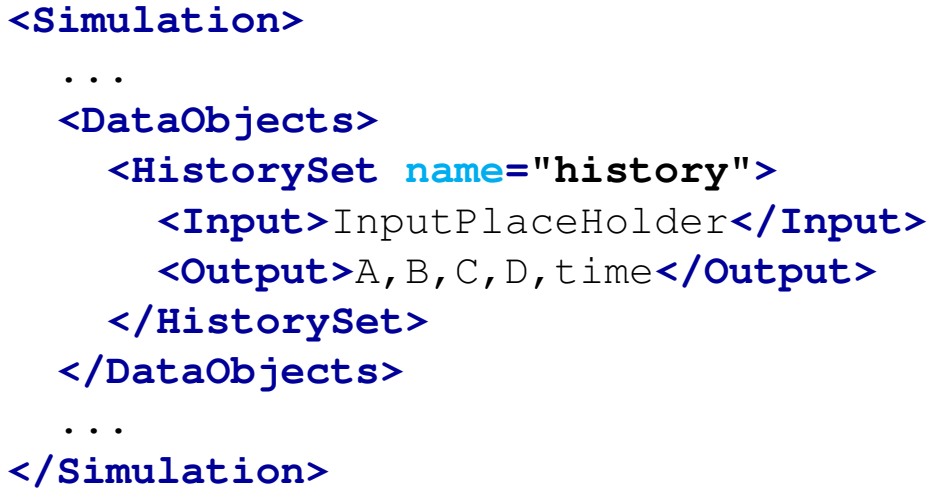

$<$ HistorySet $>$ with a user-defined identifier (e.g. "history") is used to collect the mass evolutions of four given isotopes, i.e. A, B, C, D. <Input> node is used to list the input parametes to which this data is connected. If there is no input data associated with this node, the ' InputPlaceHolder' can be used. <Output> is used to list the output parameters to which this data is connected. Similarly, if there is no output data associated with this node, the 'OutputPlaceHolder' can be used. This is mainly because both <Input> and <Output> nodes are required for all types of DataObjects.

\section{Print and plot input and output data: <OutStreams>}

The OutStreams node is the entity used for data exporting and dumping. The OutStreams support 2 actions:

- Print. This Out-Stream is able to print out (in a Comma Separated Value format) all the information contained in:

\section{- DataObjects}


- Reduced Order Models.

- Plot. This Out-Stream is able to plot 2-Dimensional, 3-Dimensional, 4-Dimensional (using color mapping) and 5-Dimensional (using marker size). Several types of plot are available, such as scatter, line, surfaces, histograms, pseudo-colors, contours, etc.

In this case, a simple <outStreams> is used to output the mass evolutions of all four model variables into a CSV file with the name prefix "print_history".

raven/tests/framework/user_guide/ravenTutorial/singleRun.xml

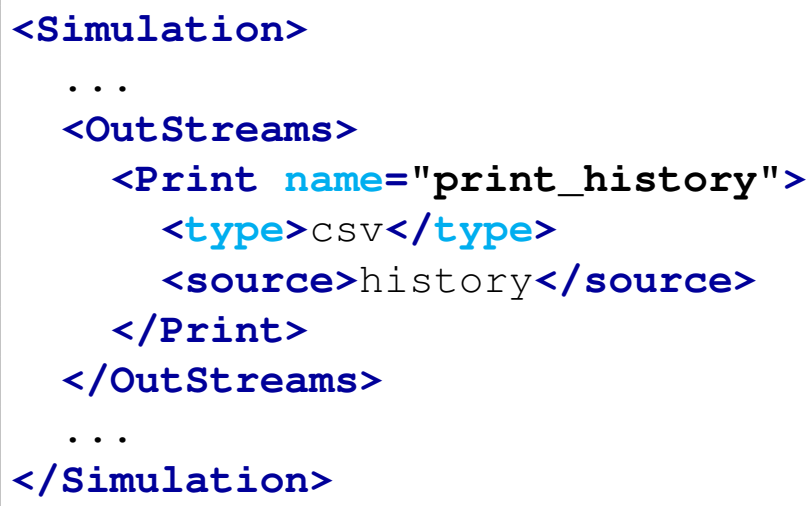

\section{Control of executions: <Steps>}

The Steps entity is used to create a peculiar analysis flow via combining together different RAVEN entities. It is the location where all the defined entities get finally linked in order to perform a combined action on a certain Model. In order to perform this linking, each entity defined in the Step needs to "play" a role:

- Input represents the input of the step. The allowable input objects depend on the type of Model in this step.

- Model represents a physical or mathematical system or behavior. The object used in this role defines the allowable types of inputs and outputs usable in this step.

- Output defines where to collect the results of an action performed by the Model. It is generally one of the following types: DataObjects, Databases, or OutStreams.

- Sampler defines the sampling strategy to be used to probe the model. Note: When a sampling strategy is employed, the "variables" defined in the <variable $\rangle$ blocks are going to be directly placed in the output objects of type DataObjects and Databases.

- Function is an extremely importance role. It introduces the capability to perform pre or post processing of model inputs and outputs. Its specific behavior depends on the step is using it.

- $R O M$ defines an acceleration reduced order model to use for a step.

- SolutionExport, represents the container of the eventual output of a step. It is the entity that is used to export the solution of a Sampler or post-processors. 
Currently, RAVEN supports the following types of <steps $>$ :

- SingleRun, perform a single run of a model

- MultiRun, perform multiple runs of a model

- RomTrainer, perform the training of a Reduced Order Model (ROM)

- PostProcess, post-process data or manipulate RAVEN entities

- IOStep, step aimed to perform multiple actions:

- construct/update a Database from a DataObjects and vice-versa

- construct/update a Database or a DataObjects object from CSV files

- stream the content of a Database or a DataObjects out through an OutStream

- store/retrieve a ROM to/from an external File using Pickle module of Python

For this example, the <SingleRun $>$ is used to assemble a calculation flow, i.e. perform a single action of a model.

raven/tests/framework/user_guide/ravenTutorial/singleRun.xml

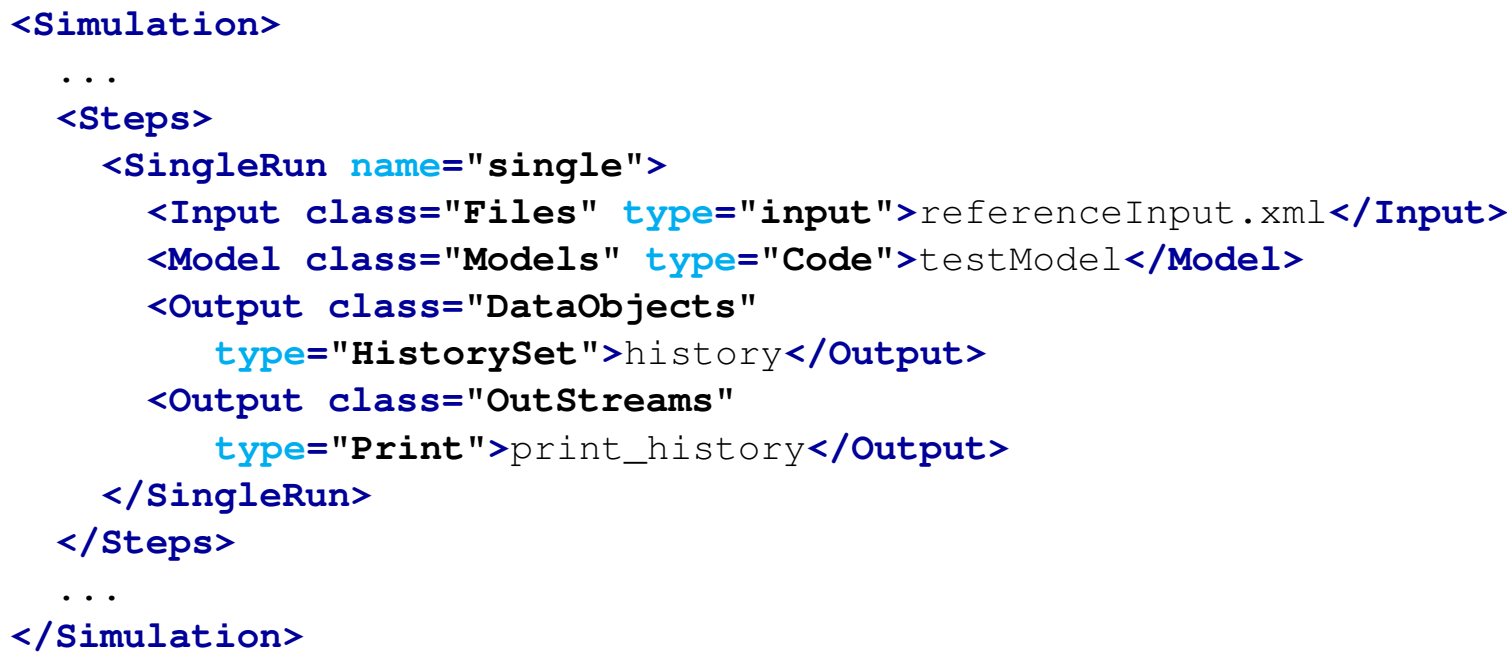

The code "testModel" will be executed once, and the outputs will be collected into a 'Dataobjects' of type HistorySet. In addition, ' OutStreams' is used to print the output data into a CSV file.

The core of the RAVEN calculation flow is the Steps system. The Steps is in charge of assembling different entities in RAVEN in order to perform a task defined by the kind of step being used (see Figure. 4). 


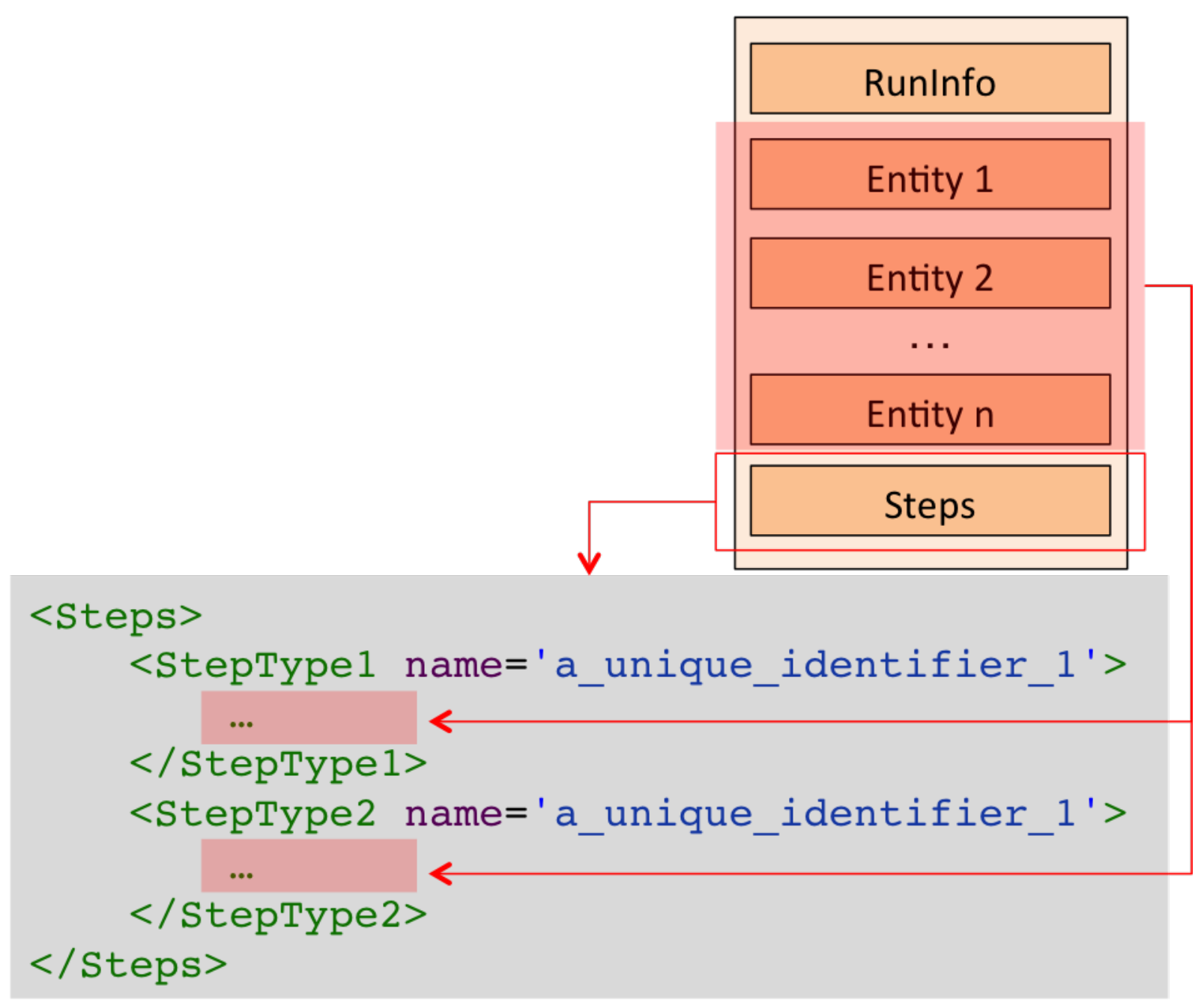

Figure 4. Example of the Steps Entity and its connection in the input file.

\subsection{Build RAVEN Input: <IOStep>}

The <IOStep> acts as a "transfer network" among different RAVEN storing or streaming objects. The number of <Input> and <Output> is unlimited. This <IOStep> assumes one-to-one mapping, i.e. the first <Input> is going to be used for the first <Output>, etc. Note: If the <Output> nodes are class 'OutStreams', the user does not need to follow this assumption, since OutStreams objects are already linked to DataObjects in the relative RAVEN input block. The IOStep can be used to:

- construct/update a Database from a DataObjects object, and vice versa; 
- construct/update a DataObject from a CSV file contained in a directory;

- construct/update a Database or a DataObjects object from CSV files contained in a directory;

- stream the content of a Database or a DataObjects out through an OutStream object;

- store/retrieve a ROM to/from an external File using Pickle module of Python.

The last function can be used to create and store mathematical model of fast solution trained to predict a response of interest of a physical system. This model can be recovered in other simulations or used to evaluate the response of a physical system in a Python program by the implementing of the Pickle module.

\subsubsection{Perform input/output operations}

In this case, we will use <IOStep> to stream the output data from the DataObjects out through the OutStreams. The <IOStep> block is shown as follows:

raven/tests/framework/user_guide/ravenTutorial/singleRunPlotAndPrint.xml

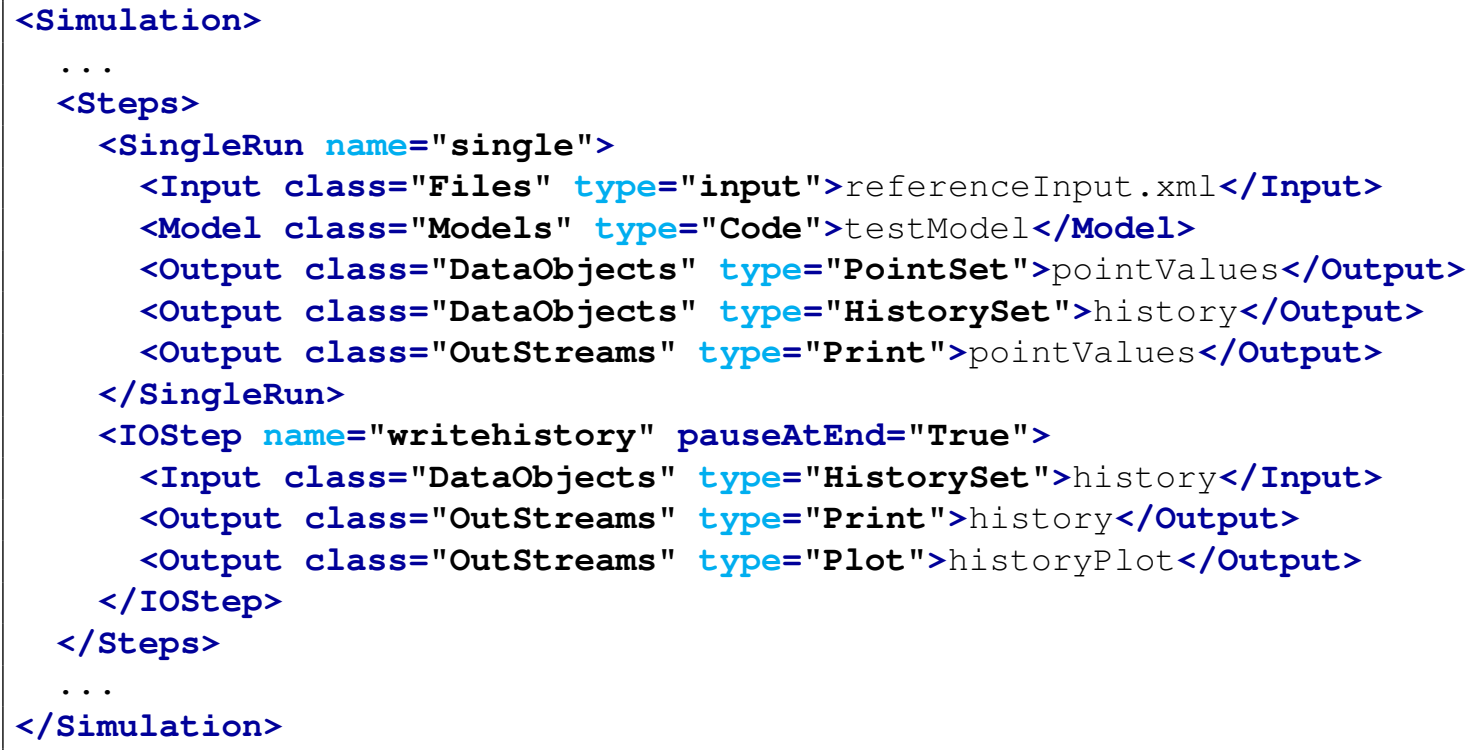

As shown in the <IOStep>, the input is a history set "history" that is previous generated by the SingleRun step. The data stored in the "history" will be printed and plotted via the OutStreams.The data object "history" is defined as follows:

raven/tests/framework/user_guide/ravenTutorial/singleRunPlotAndPrint.xml

$<$ Simulation $>$ 


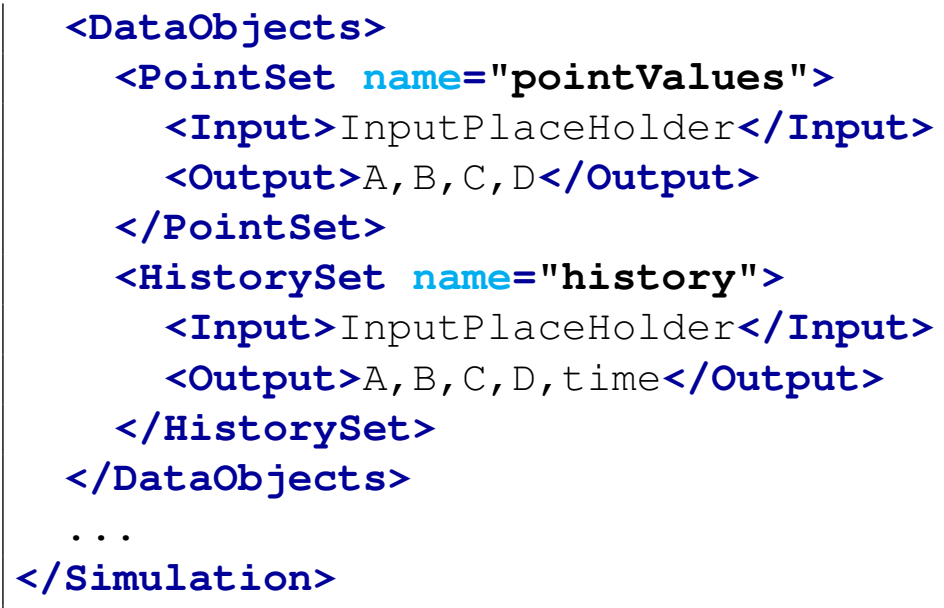

Note: If a PointSet data object is used to collect the temporal output data, only the data from the last time step will be stored in this data object. As demonstrated in this case, the output csv file with name "pointValues.csv" generated through the OutStreams in SingleRun step only contains the data for the last time step. This file can be found in the working directory specified by sub-node $<$ workingDir $>$ under node <RunInfo>.

As mentioned before, OutStreams can be used to plot the data stored in the data objects. The following input block demonstrates the use of OutStreams for plotting.

raven/tests/framework/user_guide/ravenTutorial/singleRunPlotAndPrint.xml

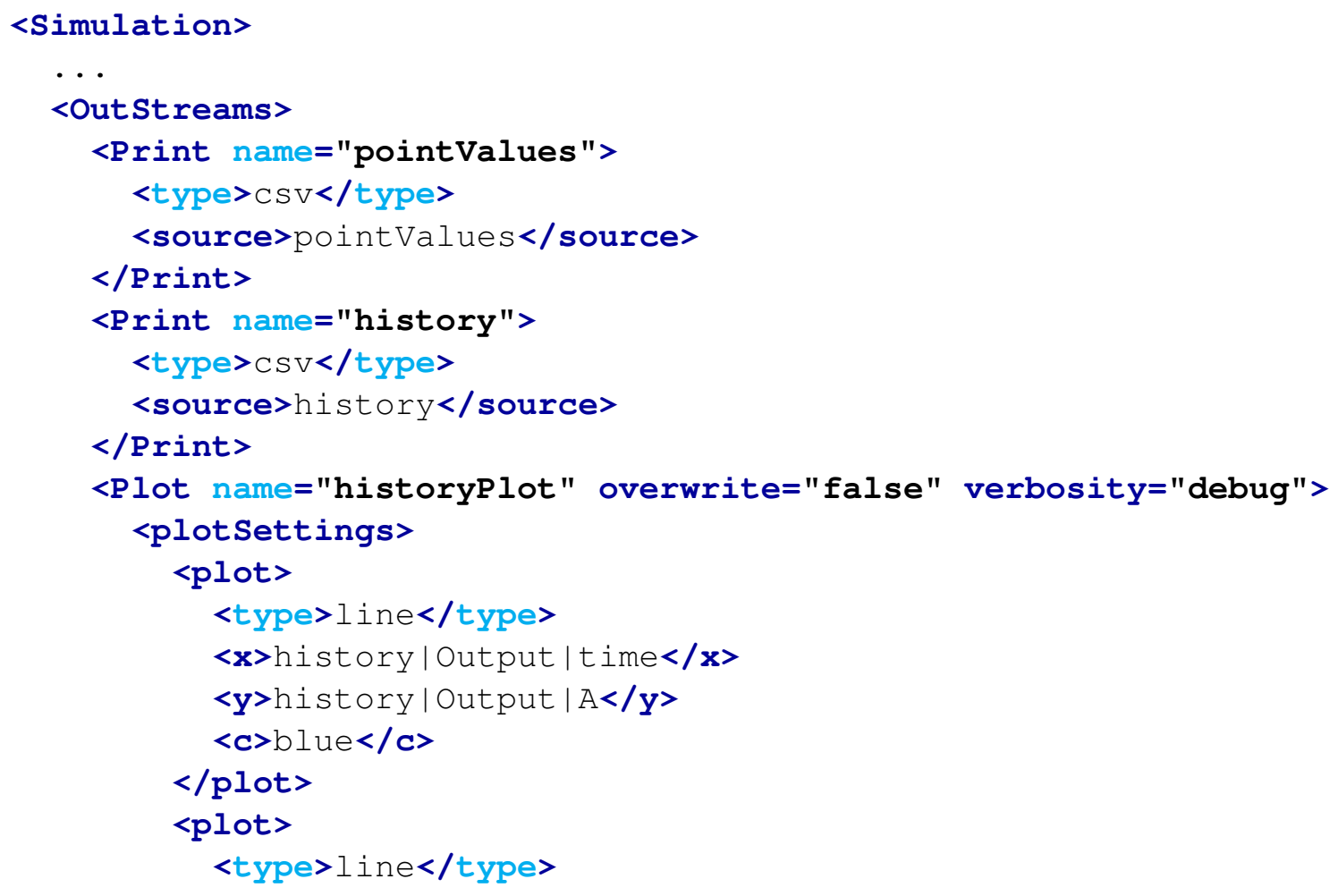




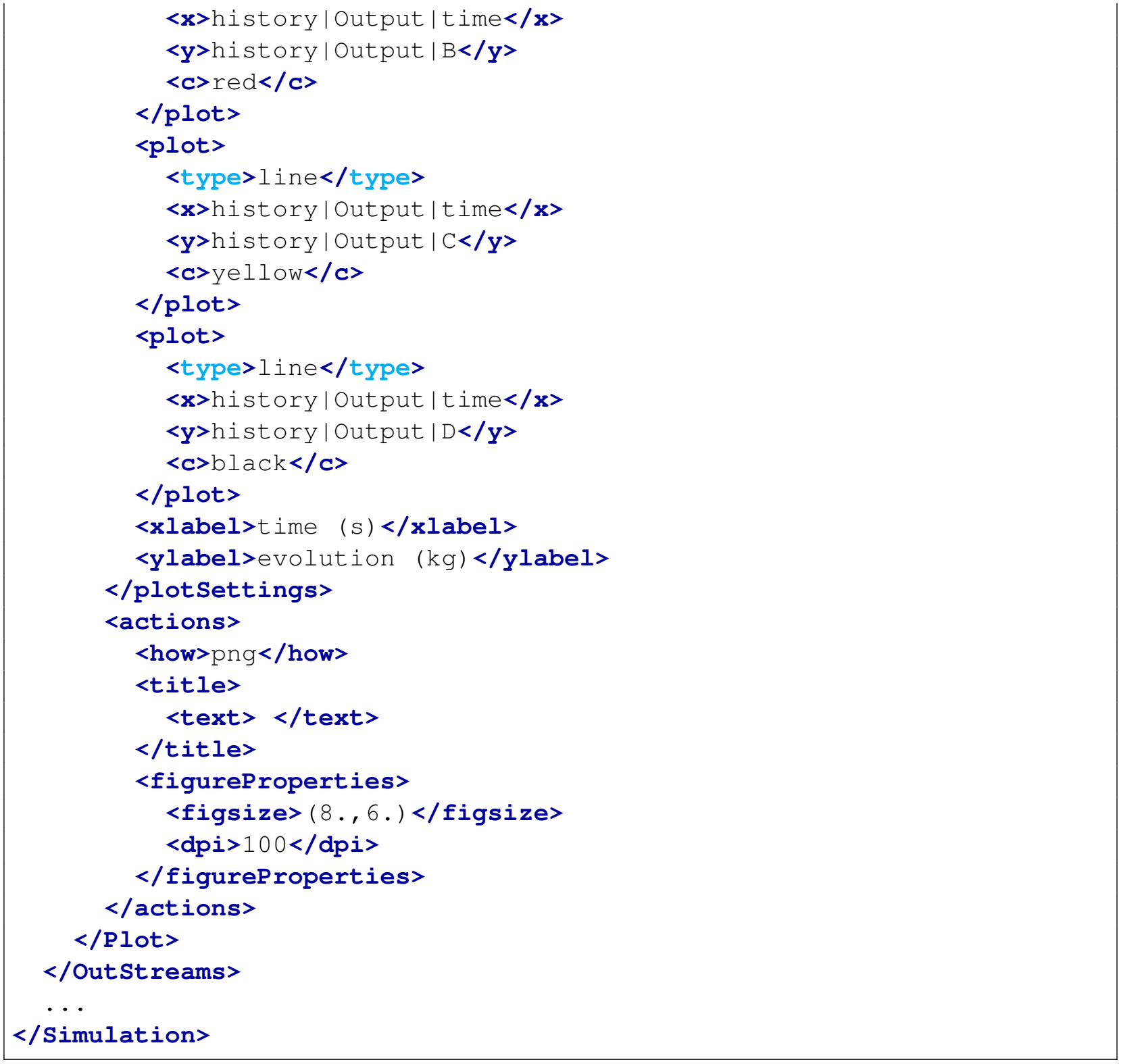

In this block, both the Out-Stream types are constructed:

- Print: named "history" connected with the DataObjects Entity "history" (<source>) When this object get used, all the information contained in the linked DataObjects are going to be dumped in CSV files (<type>).

- Plot: a single <Plot $>$ Entity is defined, containing the line plots of the 4 output variables $(A, B, C, D)$ in the same figure. This object is going to generate a PNG file in the working directory.

For examples of the numerical data produced by the OutStreams Print, see history_0 . CSV 


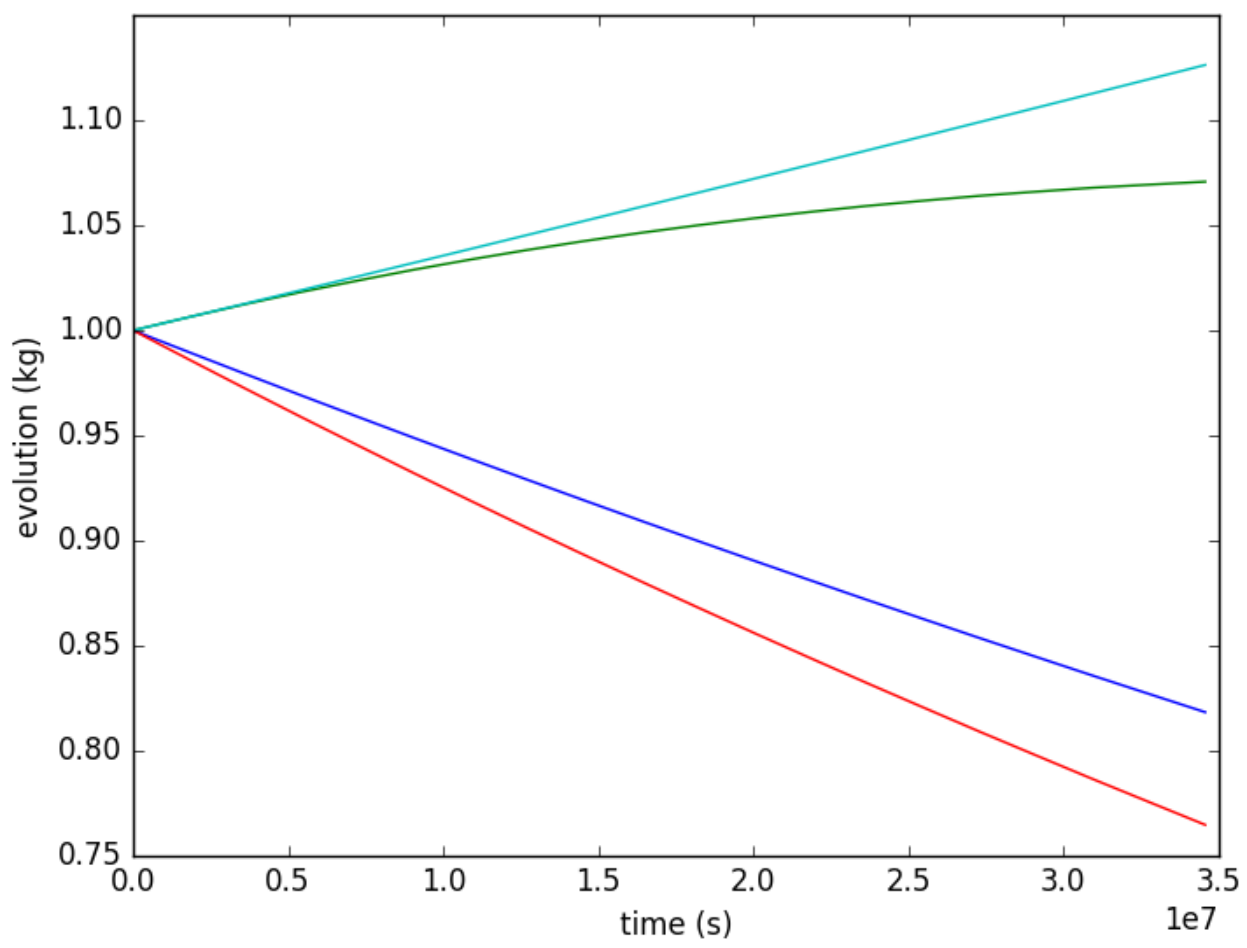

Figure 5. Plot of the history for variables $A, B, C, D$.

in the directory raven/tests/framework/user_guide/ravenTutorial/gold/singleRunPlot As previously mentioned, Figure 5 reports the four plots (four variables) drawn in the same picture.

\subsubsection{Sub-plot and selectively printing.}

This section shows how to use RAVEN to create sub-plots (multiple plots in the same figure) and how to select only some variable from the DataObjects in the Print OutStream. The goals of this Section are about learning how to:

1. Print out what contained in the DataObjects, selecting only few variables

2. Generate sub-plots (multiple plots in the same figure) of the code results

To accomplish these tasks, the <IOStep> needs to be modified as follows:

raven/tests/framework/user_guide/ravenTutorial/singleRunSubPlotsAndSelectivePrint.xml 


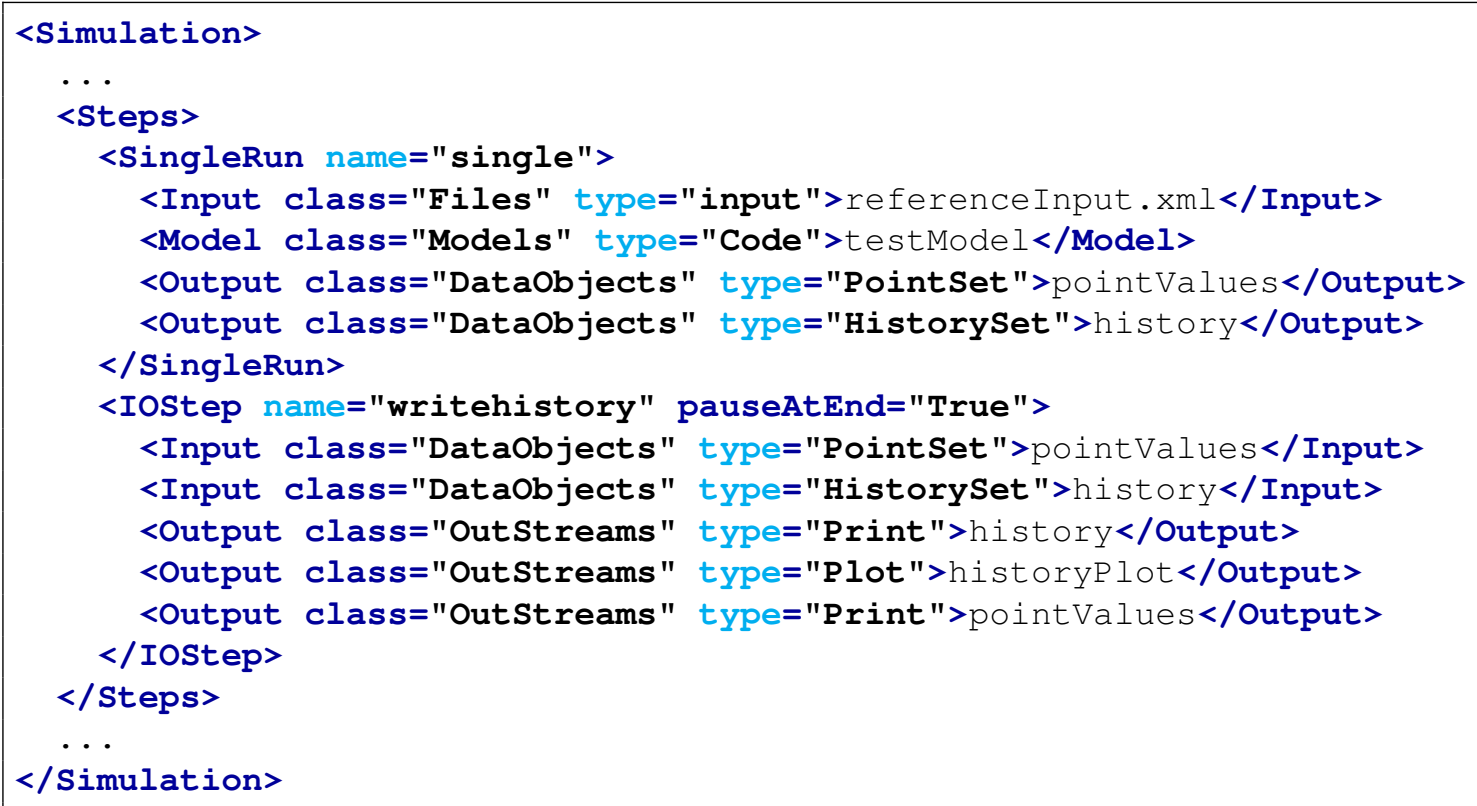

Note: as mentioned before, this <IOStep> does not need to follow the one-to-one mapping, since OutStreams are alreadly linked to the DataObjects. And the OutStreams Entity in the input defined in the previous section needs to be modified as follows:

raven/tests/framework/user_guide/ravenTutorial/singleRunSubPlotsAndSelectivePrint.xml

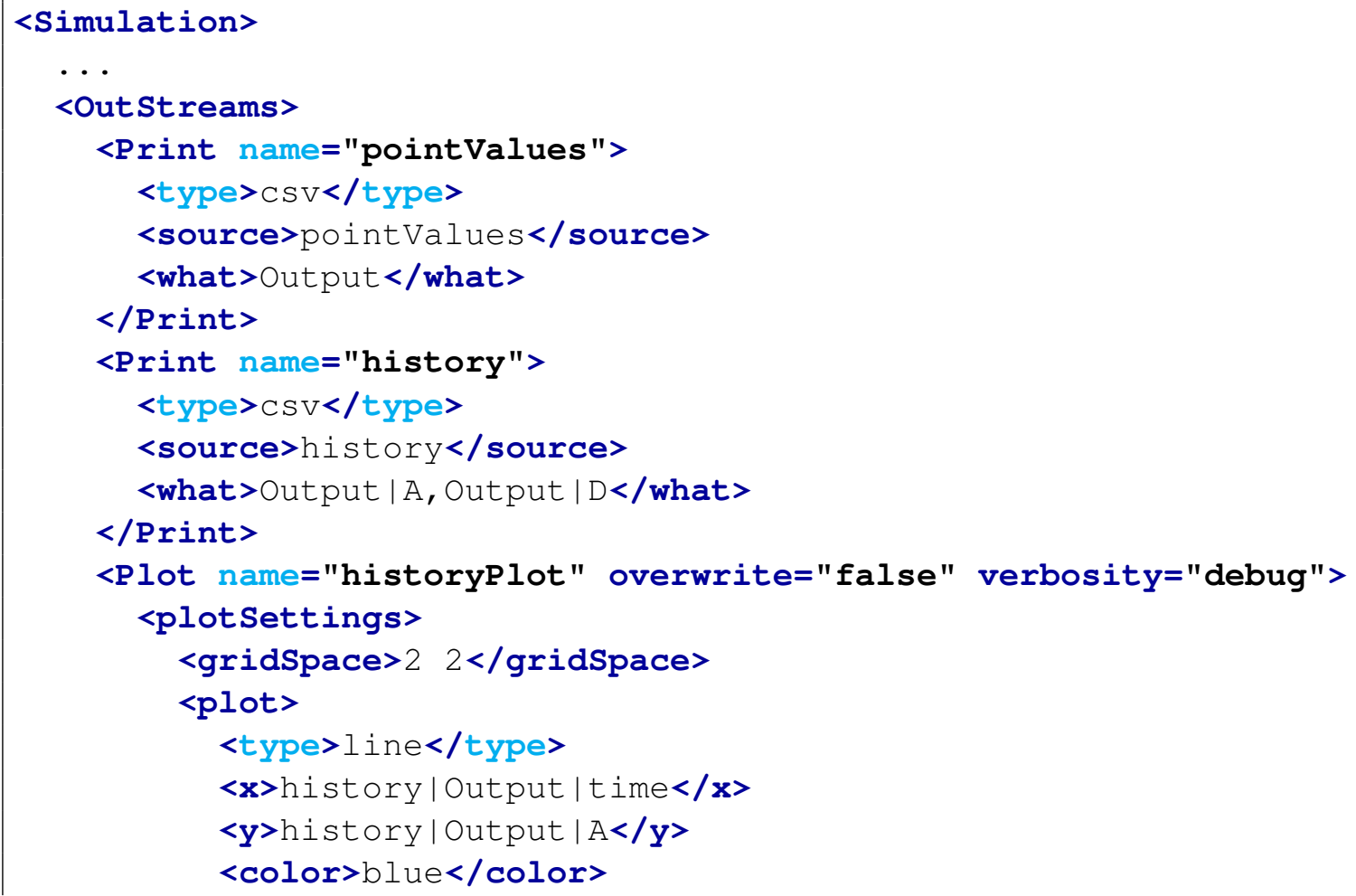




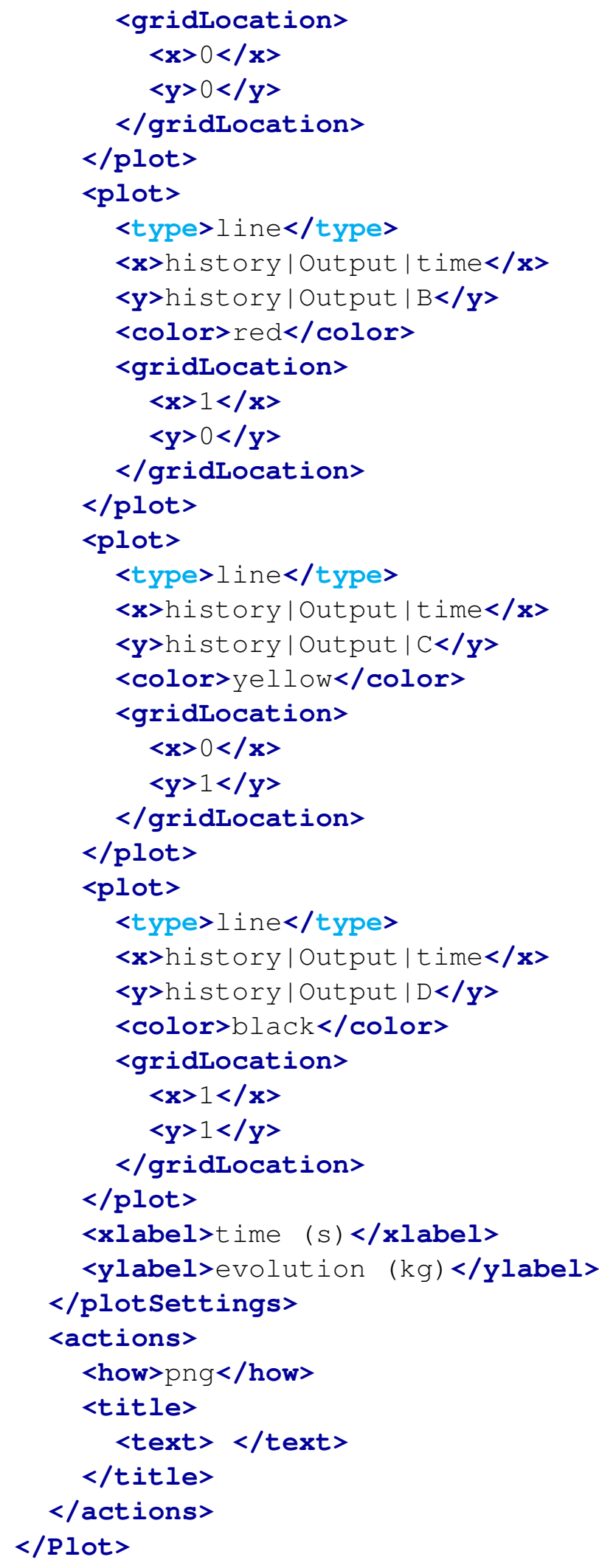


1. Print: With respect to the Print nodes defined in the previous section, it can be noticed that an additional node has been added: <what>. The Print Entity "pointValues" is going to extract and dump only the variables that are part of the Output space $(A, B, C, D$ and not InputPlaceHolder). The Print Entity "history" is instead going to print the Output space variables $A$ and $D$.

2. Plot: Note that the Plot Entity does not differ much with respect to the one in previous section: 1) the additional sub-node <gridSpace> has been added. This node is needed to define how the figure needs to be partitioned (discretization of the grid). In this case a 2 by 2 grid is requested. 2) in each <plot $>$ the node $<$ gridLocation $>$ is placed in order to specify in which position the relative plot needs to be placed. For example, in the following grid location, the relative plot is going to be placed at the bottom-right corner.

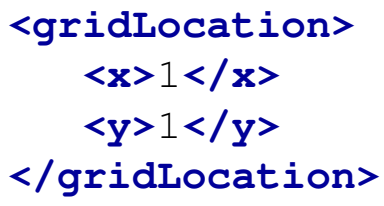

The printed data will dump to the CSV file history_0.csv, and Figure 6 reports the four plots (four variables) drawn in the same picture.

\subsection{Build RAVEN Input: <MultiRun>}

The MultiRun step allows the user to assemble the calculation flow of an analysis that requires multiple "runs" of the same model. This step is used, for example, when the input (space) of the model needs to be perturbed by a particular sampling strategy. In the <MultiRun> input block, the user needs to specify the objects that need to be used for the different allowable roles. This step accepts the following roles:

- Input

- Model

- Output

- Sampler 

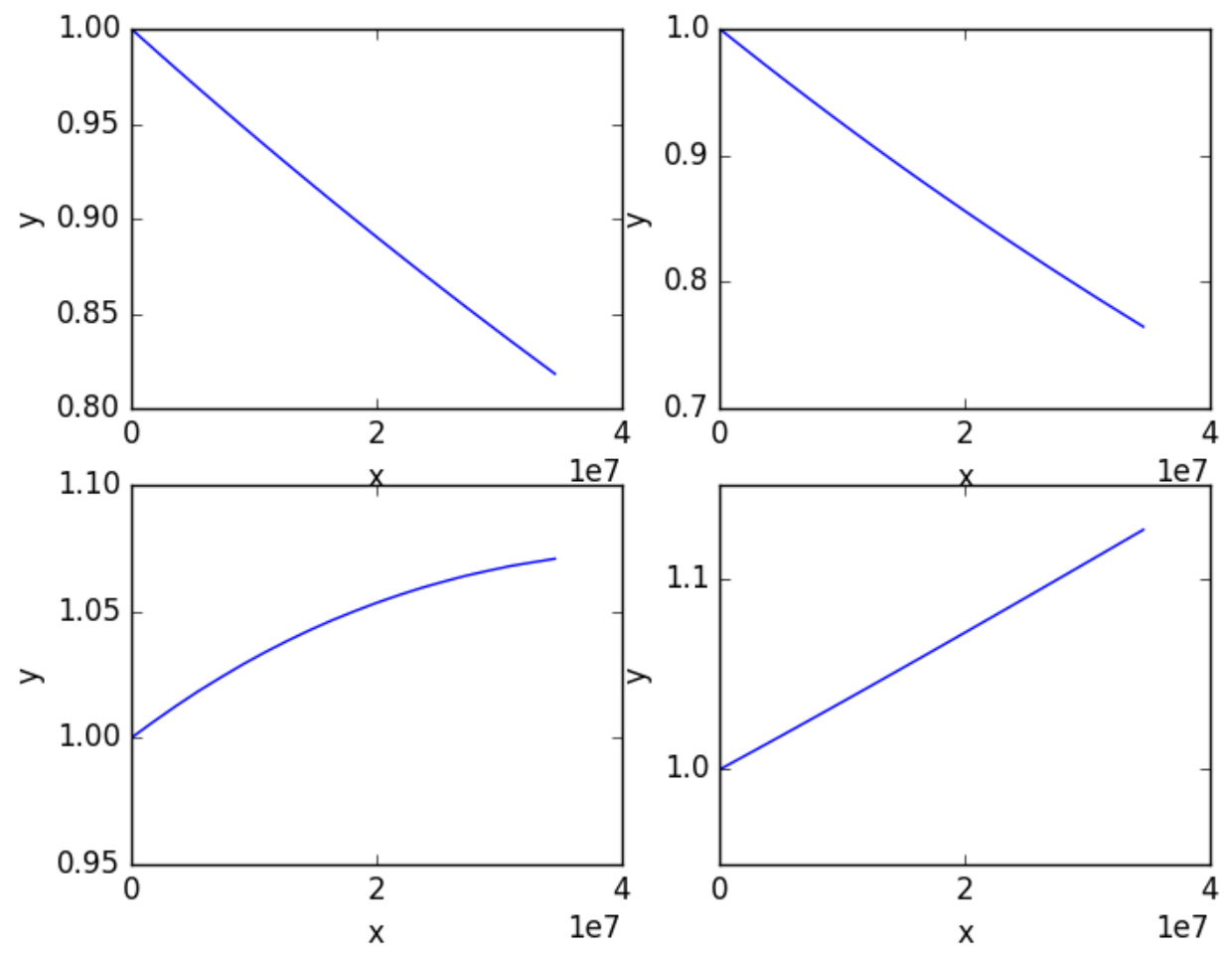

Figure 6. Subplot of the history for variables $A, B, C, D$.

\section{- Optimizer}

- SolutionExport

MultiRun is intended to handle calculations that involve multiple runs of a driven code (sampling strategies). Firstly, the RAVEN input file associates the variables to a set of PDFs and to a sampling strategy. The "multi-run" step is used to perform several runs in a block of a model (e.g. in a MC sampling).

As shown in Figure 7, at the beginning of each sub sequential run, the sampler provides the new values of the variables to be perturbed. The code API places those values in the input file. At this point, the code API generates the run command and asks to be queued by the job handler. The job handler manages the parallel execution of as many runs as possible within a user prescribed range and communicates with the step controller when a new set of output files are ready to be processed. The code API receives the new input files and collects the data in the RAVEN internal format. The sampler is queried to assess if the sequence of runs is ended, if not, the step controller asks for a new set of values from the sampler and the sequence is restarted. The job handler is currently 


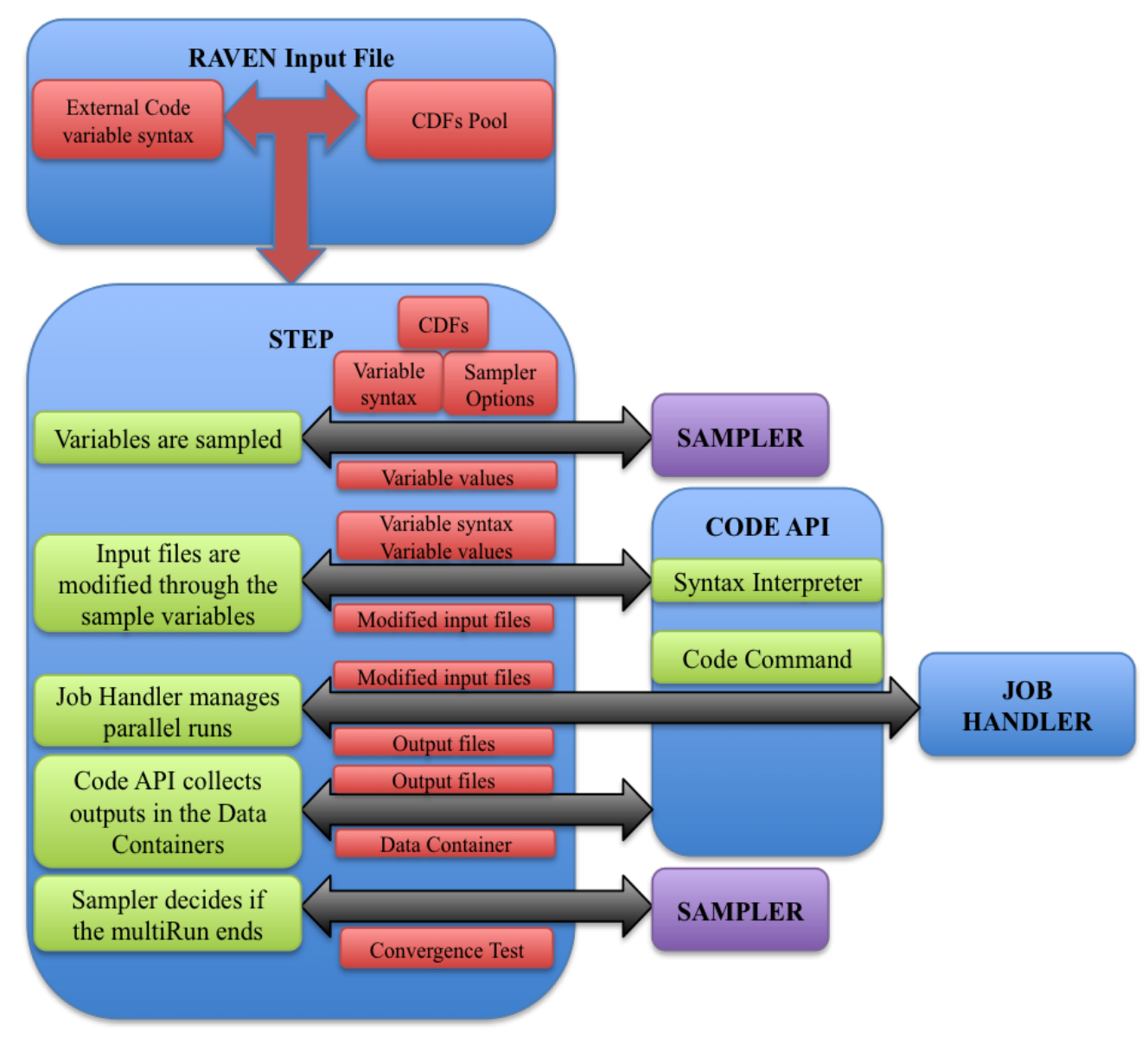

Figure 7. Calculation flow for a multi-run sampling

capable to run different run instances of the code in parallel and can also handle codes that are multi-threaded or using any form of parallel implementation. RAVEN also has the capability to plot the simulation outcomes while the set of sampling is performed and to store the data for later recovery.

In this section, we will show the user how to set up the Sampler, and employ MultiRun to execute all perturbed models. The use of Optimizer and SolutionExport will be introduced in another section. The Samplers entity is the container of all the algorithms designed to perform the perturbation of the input space. The Samplers can be categorized into three main classes:

- Forward. Sampling strategies that do not leverage the information coming from already evaluated realizations in the input space. For example, Monte-Carlo, Stratified (LHS), Grid, Response Surface, Factorial Design, Sparse Grid, etc.

- Adaptive. Sampling strategies that take advantages of the information coming from already 
evaluated realizations of the input space, adapting the sampling strategies to key figures of merits. For example, Limit Surface search, Adaptive sparse grid, etc.

- Dynamic Event Tree. Sampling strategies that perform the exploration of the input space based on the dynamic evolution of the system, employing branching techniques. For example, Dynamic Event Tree, Hybrid Dynamic Event Tree, etc.

The sampler is probably the most important entity in the RAVEN framework. It provides many different sampling strategies that can be used in almost all RAVEN related applications. In this section, we will only illustrate the simplest forward sampler, i.e. Monte-Carlo, to familarize the user with the use of sampler. Monte-Carlo method is one of the most-used methodologies in several mathematic disciplines. The theory of this method can be found in the RAVEN theory manual. In addition, we will continue to use the AnalyticBateman to illustrate the setup of MultiRun and Samplers. In order to accomplish these tasks, the following precedures or RAVEN entities are needed:

1. Set up the running environment: <RunInfo>

2. Provide the required files: $\langle$ Files $>$

3. Link between RAVEN and dirven code: <Models>

4. Define probability distribution functions for inputs: <Distributions>

5. Set up a simple Monte-Carlo sampling for perturbing the input space: <Samplers>

6. Store the input and output data: <DataObjects>

7. Print and plot input and output data: <Outstreams>

8. Control multiple executions: <Steps>

In section 2.2, we have already discussed the use of <RunInfo $\rangle,\langle$ Files $\rangle,\langle$ Models $\rangle$, $<$ DataObjects $>$, <OutStreams $>$. For practice, the user can try to build these RAVEN entities by themselves, and refer to the complete input file located at raven/tests/framework/user_guide/ ravenTutorial/MonteCarlo.xml. In this section, we'd like to show the users how to set up <Distributions $>$, <Samplers> and MultiRun of <Steps>. RAVEN employs <Distributions> to define many different probability distribution functions (PDFs) that can be used to characterize the input parameters. One can consider the $\langle$ Distributions $>$ entity to be a container of all the stochastic representation of random variables. Currently, RAVEN supports:

- 1-Dimensional continuous and discrete distributions, such as Normal, Weibull, Binomial, etc. 
- $N$-Dimensional distributions, such as Multivariate Normal, user-inputted N-Dimensional distributions.

For the AnalyticBateman example, two 1-D uniform distributions are defined:

- sigma $\sim \mathbb{U}(1,10)$, used to model the uncertainties associated with the sigma Model variables;

- decayConstant $\sim \mathbb{U}(0.5 e-8,1 e-8)$, used to model the uncertainties associated with the Model variable decay constants. Note that the same distribution can be re-used for multiple input variables, while still keeping those variables independent.

The following is the definition of $<$ Distributions $>$ block that is used for the AnalyticBateman problem:

raven/tests/framework/user_guide/ravenTutorial/MonteCarlo.xml

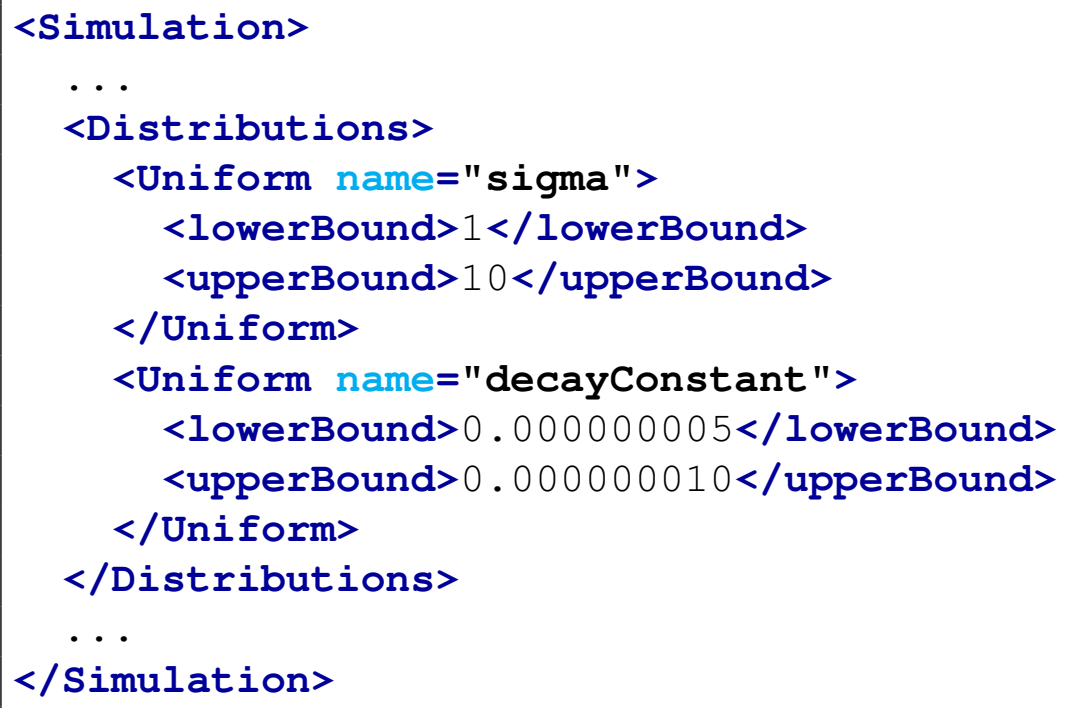

For uniform distributions, only <lowerBound> and <upperBound> are required. For other distributions, please refer to the RAVEN user manual.

As we already mentioned, we will employ Monte-Carlo sampling strategy to demonstrate MultiRun. To employ the Monte-Carlo sampling strategy, a <MonteCarlo> node needs to be defined. The user also needs to specify the variables that need to be sampled using <variable>. In addition, the setting for this sampler need to be specified in the <samplerInit> block. The only required sub-node $\langle$ limit $>$ is used to specify the number of Monte Carlo samples. The user can also use other optional sub-node to characterize their samplers. For this example, the <Samplers> block is:

raven/tests/framework/user_guide/ravenTutorial/MonteCarlo.xml 


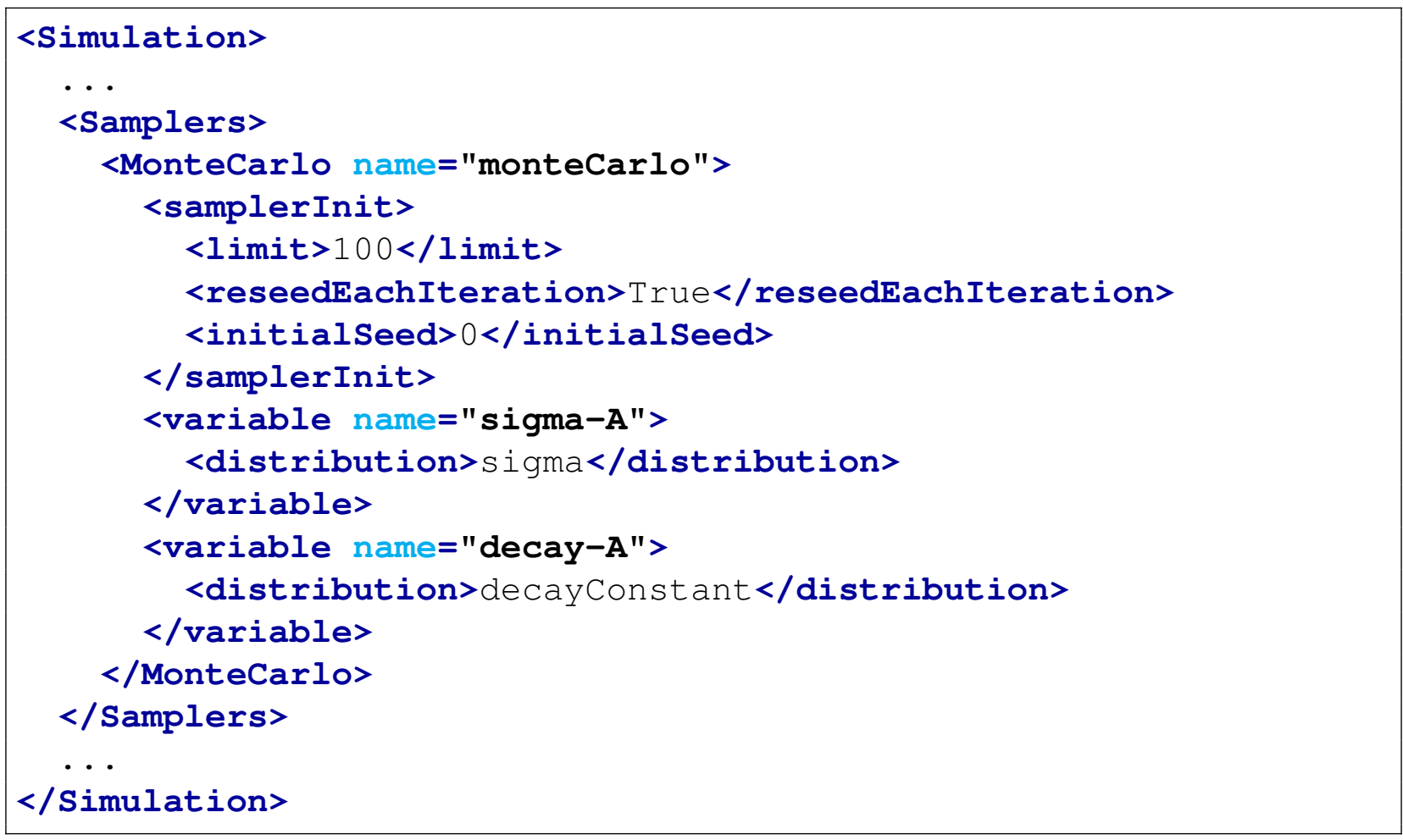

In this case, the Monte-Carlo method is employed on two model variables, each of which are listed by name and are associated with a distribution. Note that the decay- and sigma-variables are associated with the distributions decayConstant and sigma, respectively. These variables and their values are passed to the model via the generic code interface. This requires the users to make some changes in their input files in order to accept these variables. For example, the input file of AnalyticBateman becomes:

raven/tests/framework/user_guide/ravenTutorial/commonFiles/referenceInput_generic_CI.xml

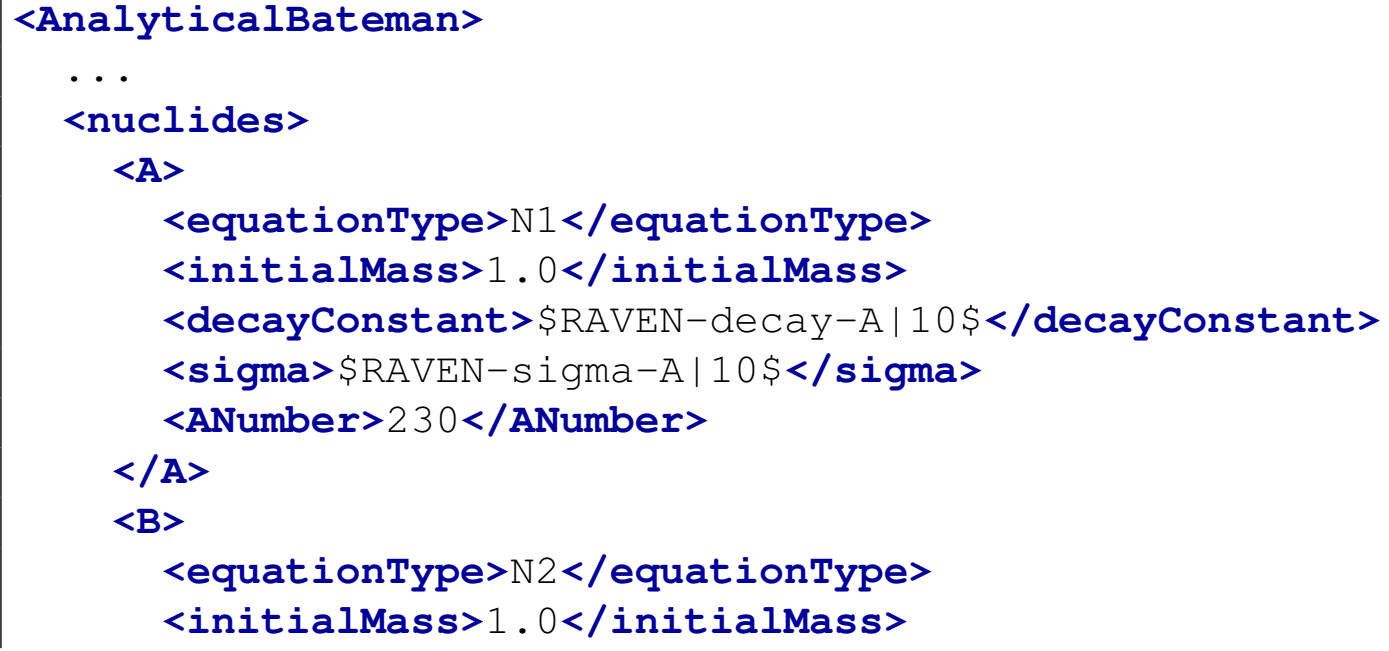




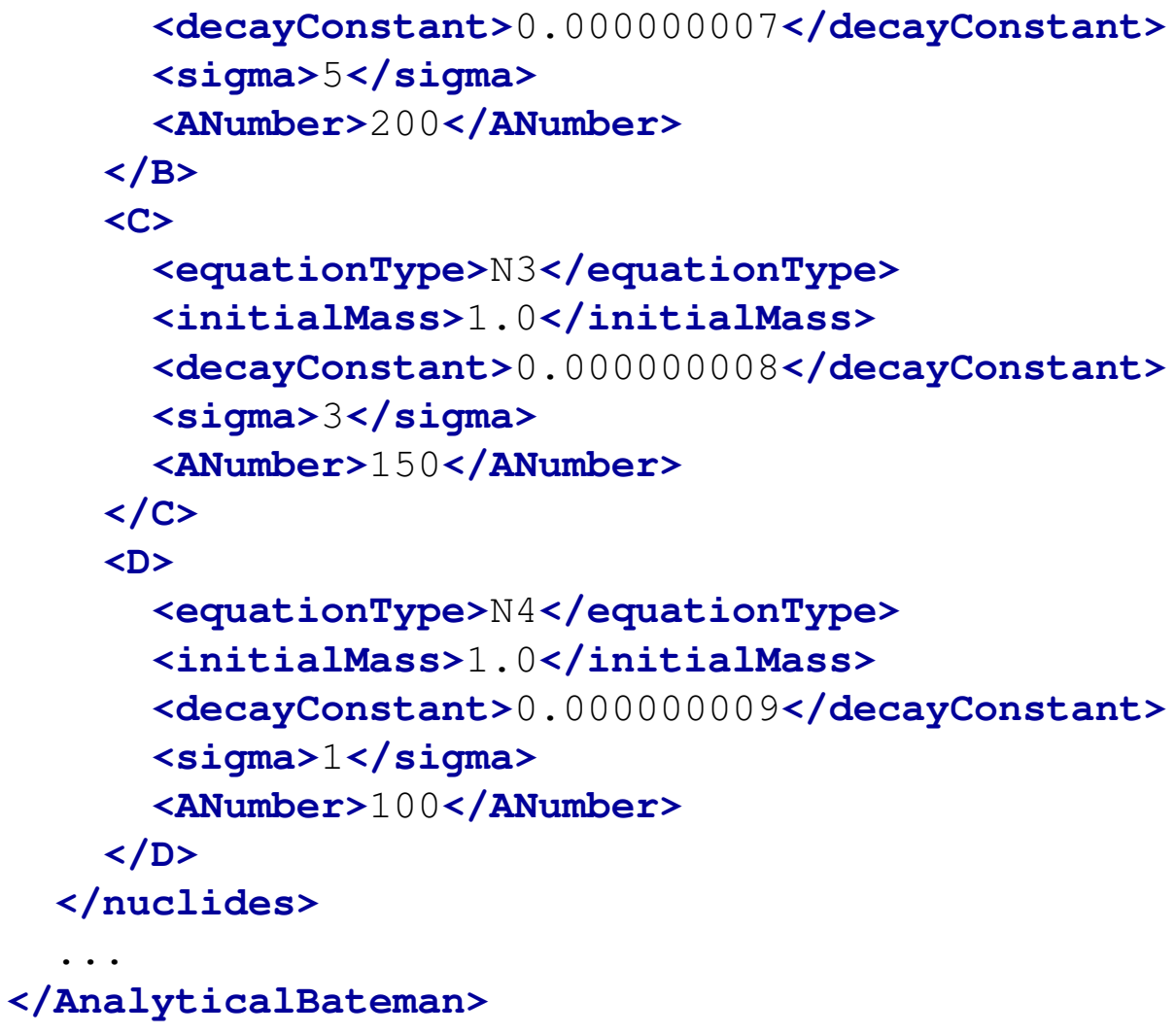

As shown in this example, the values of nodes <sigma> and <decayconstant> are replaced with variables ' \$RAVEN-decay-A|10\$' and '\$RAVEN-sigma-A|10\$', respectively. Note: we use prefix 'RAVEN-' +'variable names defined inside RAVEN input files' within ' $\$ \mathbf{\$}$ ' to define the RAVEN-editable input parameters. In other words, the RAVEN-editable input parameters is used to transfer the sampled values of RAVEN variables to input parameters of given code. This is the only way to connect the input parameters of code with variables of RAVEN if Generic code interface is employed. In addition, we use the wild-cards I to define the format of the value of the RAVEN-editable input parameters. In this case, the value that is going to be replaced by the generic code interface will be left-justified with a string length of 10 (e.g. " 10"). Other formatting options can be found in the RAVEN user manual.

As we already mentioned, the Generic code interface requires that the codes need to return a CSV file with the input parameters and output parameters. The filename of the CSV file should includes:

- prefix: "out $"$

- filename: the base of input filename without extension

- extension: ".csv" 
For this case, the input filename is "referenceInput_generic_CI.xml", thus the output CSV filename should be "out $\sim$ referenceInput_generic_CI.csv".

Once all the other entities are defined in the RAVEN input file, they must be combined in the <steps> block, which dictates the workflow of RAVEN. For this case, two <steps> are defined:

- <MultiRun> "sample", used to run the multiple instances of the driven code and collect the outputs in the two DataObjects. As it can be seen, the <Sampler > is specified to communicate to the Step that the driven code needs to be perturbed through the Monte-Carlo sampling.

- <IOStep> named "writeHistories", used to 1) dump the "histories" and "samples" DataObjects Entity to a CSV file and 2) plot the data in the EPS file.

Figures 8 and 9 show the report generated by RAVEN of the evolution of the variable $A$ and its final values, respectively.

raven/tests/framework/user_guide/ravenTutorial/MonteCarlo.xml

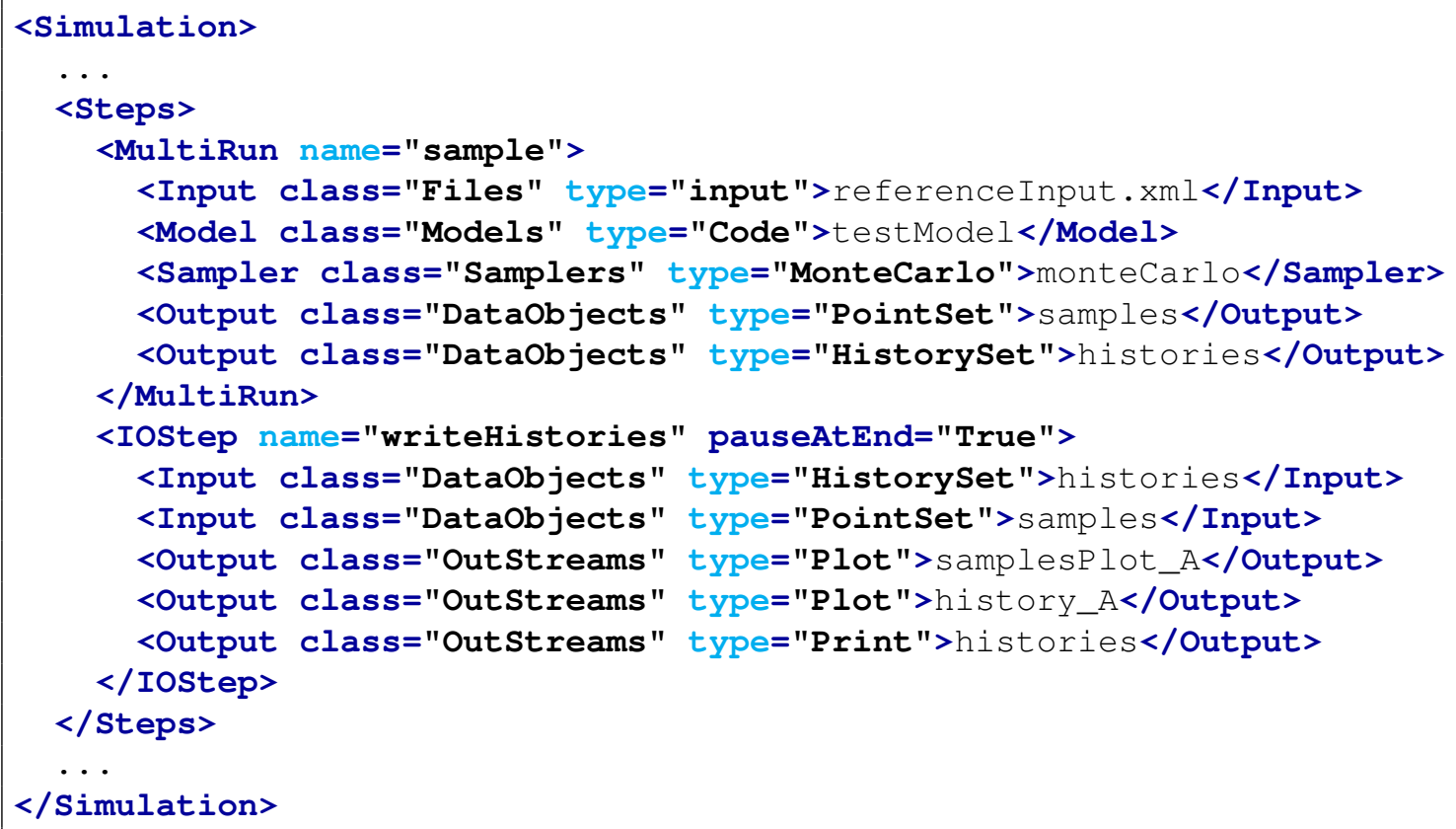




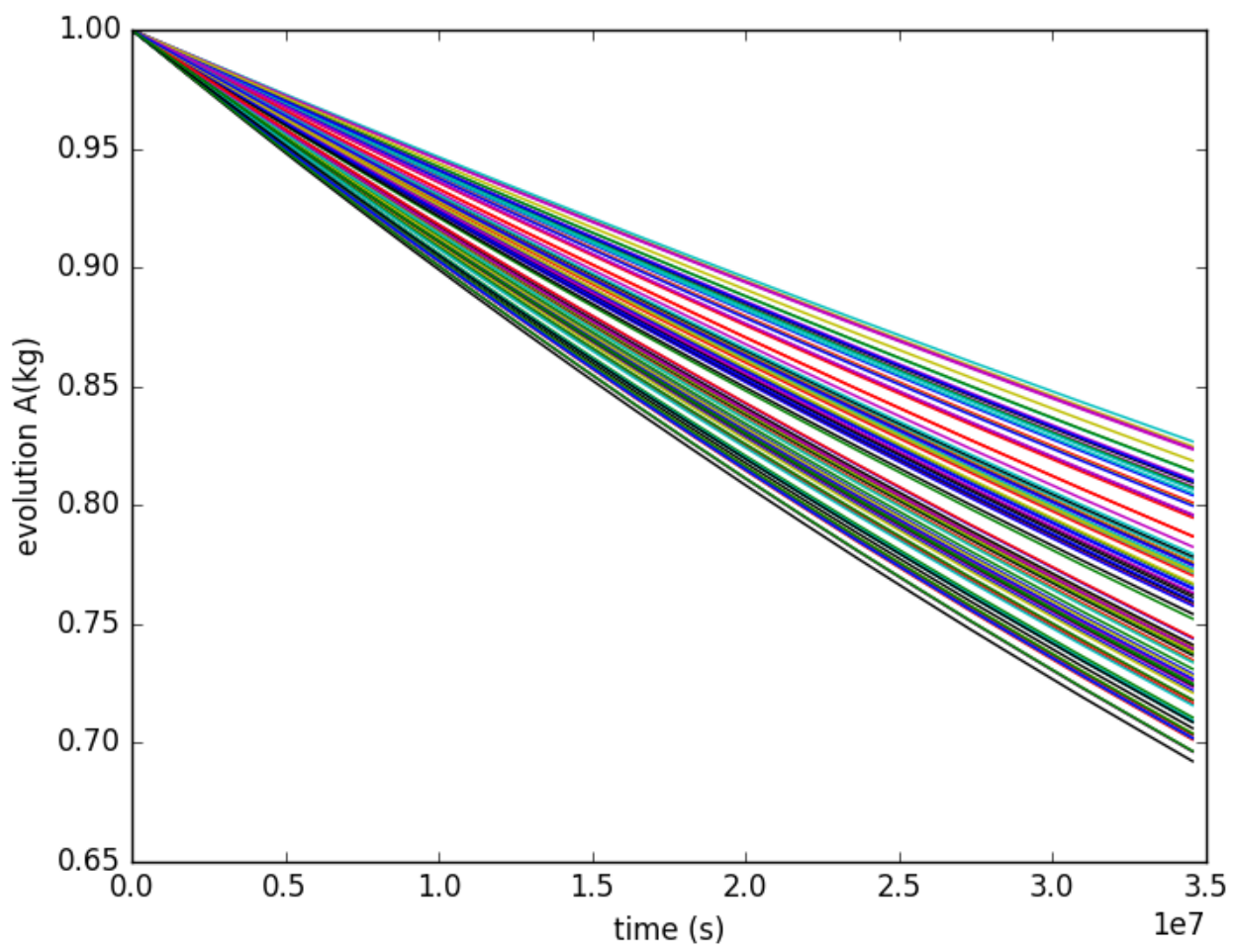

Figure 8. Plot of the histories generated by the Monte Carlo sampling for variable $A$. 


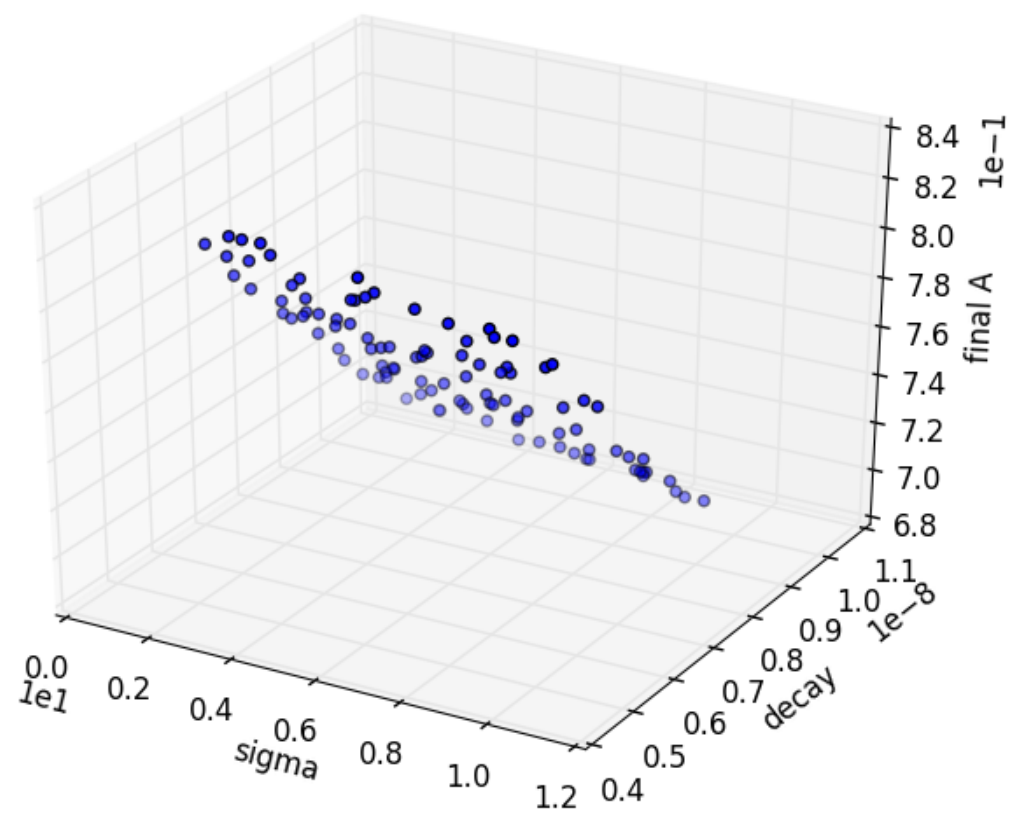

Figure 9. Plot of the samples generated by the MC sampling for variable $A$. 


\subsection{Build RAVEN Input: <RomTrainer>}

The RomTrainer step type performs the training of a Reduced Order Model (ROM), and the specifications of this step must be defined within a <RomTrainer $>$ block. ROMs, also known as a surrogate model, are used to lower the computational cost, reducing the number of needed points and prioritizing the area of the input space that needs to be explored when the simulations using the high-fidelity codes are very expensive. ROMs can be considered as an artificial representation of the link between the input and output spaces for a particular system.

In most of the cases of interest, the information that is sought is related to defining the failure boundaries of a system with respect to perturbations in the input space. For this reason, in the development of RAVEN, it has been given priority to the introduction of a class of supervised learning algorithms, which are usually referred to as classifiers. A classifier is a reduced order model that is capable of representing the system behavior through a binary response (failure/success). Currently, RAVEN supports around 40 different ROM methodologies. All these supervised learning algorithms have been imported via an API from the Scikit-Learn library. In addition, the $\mathrm{N}$-Dimensional spline and the inverse weight methods that are currently available for the interpolation of N-Dimensional PDF/CDF, can also be used as ROMs.

In this section, the $\mathrm{N}$-dimensional inverse weight method is employed to construct $\mathrm{ROM}$ to familarize the user with the use of ROMs. Inverse distance weighting (IDW) is a type of deterministic method for multivariate interpolation with a known scattered set of points. The assigned values to unknown points are calculated via a weighted average of the values available at the known points.

It is important to NOTE that RAVEN uses a Z-score normalization of the training data before constructing the NDinvDistWeight ROM:

$$
\mathbf{X}^{\prime}=\frac{(\mathbf{X}-\mu)}{\sigma}
$$

\subsubsection{How to train and output a ROM?}

In general, the "training" is a process that use sampling of the physical model to improve the prediction capability of the ROM. As mentioned before, RAVEN provides lots of different sampling strategies, such as Monte Carlo, Grid, Stratified (e.g. LHS), and Stochastic Collocation methods. All of them can be used to train a ROM. In this section, we will continue to use AnalyticBateman to illustrate the setup of RomTrainer. As before, a simple step MultiRun with Monte Carlo sampler is employed to generate the data set that can be used to train the ROM. The full RAVEN input file can be found: raven/tests/framework/user_guide/ravenTutorial/RomTrain.xml. Because the setup of MultiRun is the same as previous example in section 2.4, the RAVEN input file about the MultiRun is not include in this section. However, the full precedures are listed here to make 
the user better understand the construction of ROM. The following precedures or RAVEN entities are needed:

- MultiRun: Monte Carlo sampling to generate the data set

1. Set up the running environment: <RunInfo>;

2. Provide the required files: $\langle F$ iles $>$;

3. Link between RAVEN and dirven code: <Code>;

4. Define probability distribution functions for inputs: <Distributions $>$;

5. Set up a simple Monte-Carlo sampling for perturbing the input space: <Samplers>;

6. Store the input and output data: <DataObjects $>$;

7. Print and plot input and output data: <OutStreams>;

8. Control multiple executions: <MultiRun>.

- RomTrainer: Train the ROM with given data set

1. Specify the type of ROMs: $<$ ROM $>$;

2. Provide the data set for ROM training: <DataObjects>;

3. Train the ROM: <RomTrainer>;

4. Dump the ROM: <IOStep>

The specifications of the reduced order model must be defined within $<$ ROM $>$ XML block. This XML node accepts the following attributes:

- name, required string attribute, user-defined identifier of this model.

- subType, required string attribute, defines which of the sub-types should be used, choosing among the previously reported types.

In the $\langle$ ROM $>$ input block, the following XML sub-nodes are required, independent of the subType specified:

- <Features>, comma separated string, required field, specifies the names of the features of this ROM. Note: These parameters are going to be requested for the training of this object;

- <Target>, comma separated string, required field, contains a comma separated list of the targets of this ROM. These parameters are the Figures of Merit (FOMs) this ROM is supposed to predict. Note: These parameters are going to be requested for the training of this object. 
For each sub-type specified in the attribute subType, additional sub-nodes may be required (Please check the RAVEN user manual for each ROM). In addition, if an <Historyset> is provided in the training step, then a temporal ROM is created, i.e. a ROM that generates not a single value prediction of each element indicated in the <Target> block but its full temporal profile. In this section, a time-dependent ROM will be constructed.

In order to use $\mathbf{N}$-dimensional inverse distance weighting ROM, the $<\mathrm{ROM}>$ attribute subType needs to be 'NDinvDistWeight' . The following addition sub-node is also required.

- $\langle\mathrm{p}\rangle$, integer, required field, must be greater than zero and represents the "power parameter". For the choice of value for $\langle\mathrm{p}\rangle$, it is necessary to consider the degree of smoothing desired in the interpolation/extrapolation, the density and distribution of samples being interpolated, and the maximum distance over which an individual sample is allowed to influence the surrounding ones (lower $p$ means greater importance for points far away).

Based on previous RAVEN input file used in section 2.4, the <ROM $>$, <RomTrain $>$ and $<$ IOStep $>$ are added. The $<$ ROM $>$ is used to describe the N-dimensional inverse distance weighting ROM:

raven/tests/framework/user_guide/ravenTutorial/RomTrain.xml

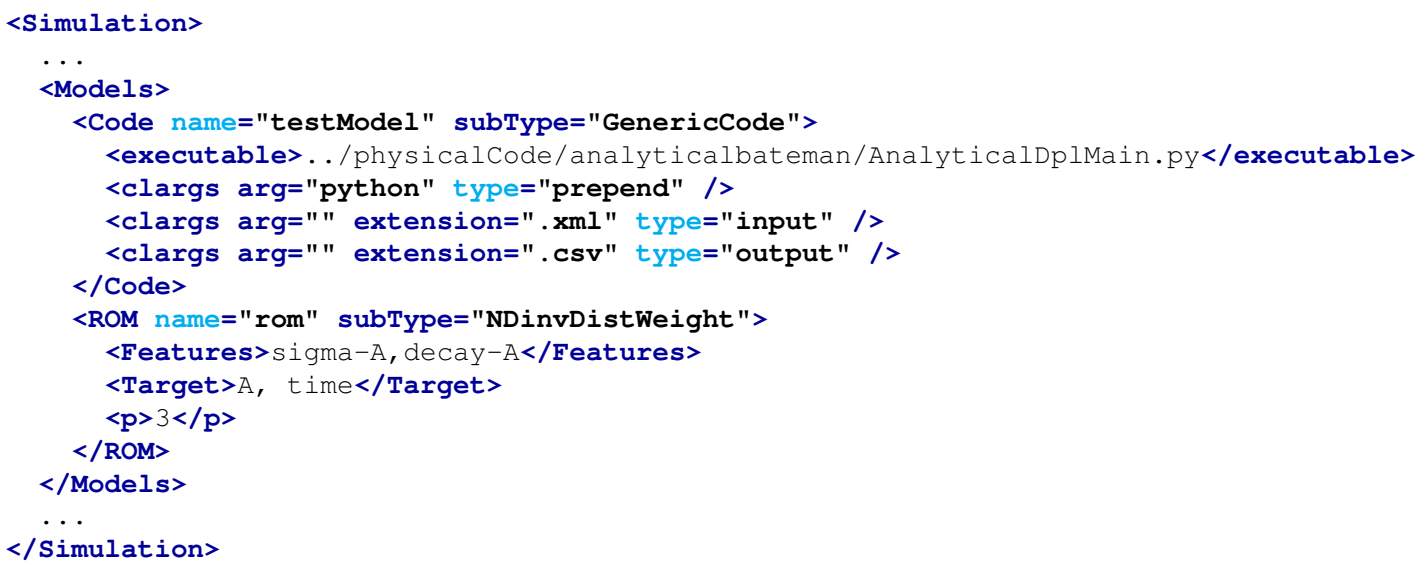

The inputs and outputs of AnalyticBateman is used to generate the data set. The ROM will be constructed considering two features (sigma- $A$ and decay- $A$ ) and two targets ( $A$ and time). Note: the time is treated as target in ROM construction.

Then, the <RomTrain $>$ and <IOStep $>$ are used to construct ROM on the fly or dump the ROM into a file (i.e. pickled ROM), respectively.

raven/tests/framework/user_guide/ravenTutorial/RomTrain.xml

$<$ Simulation $>$ 


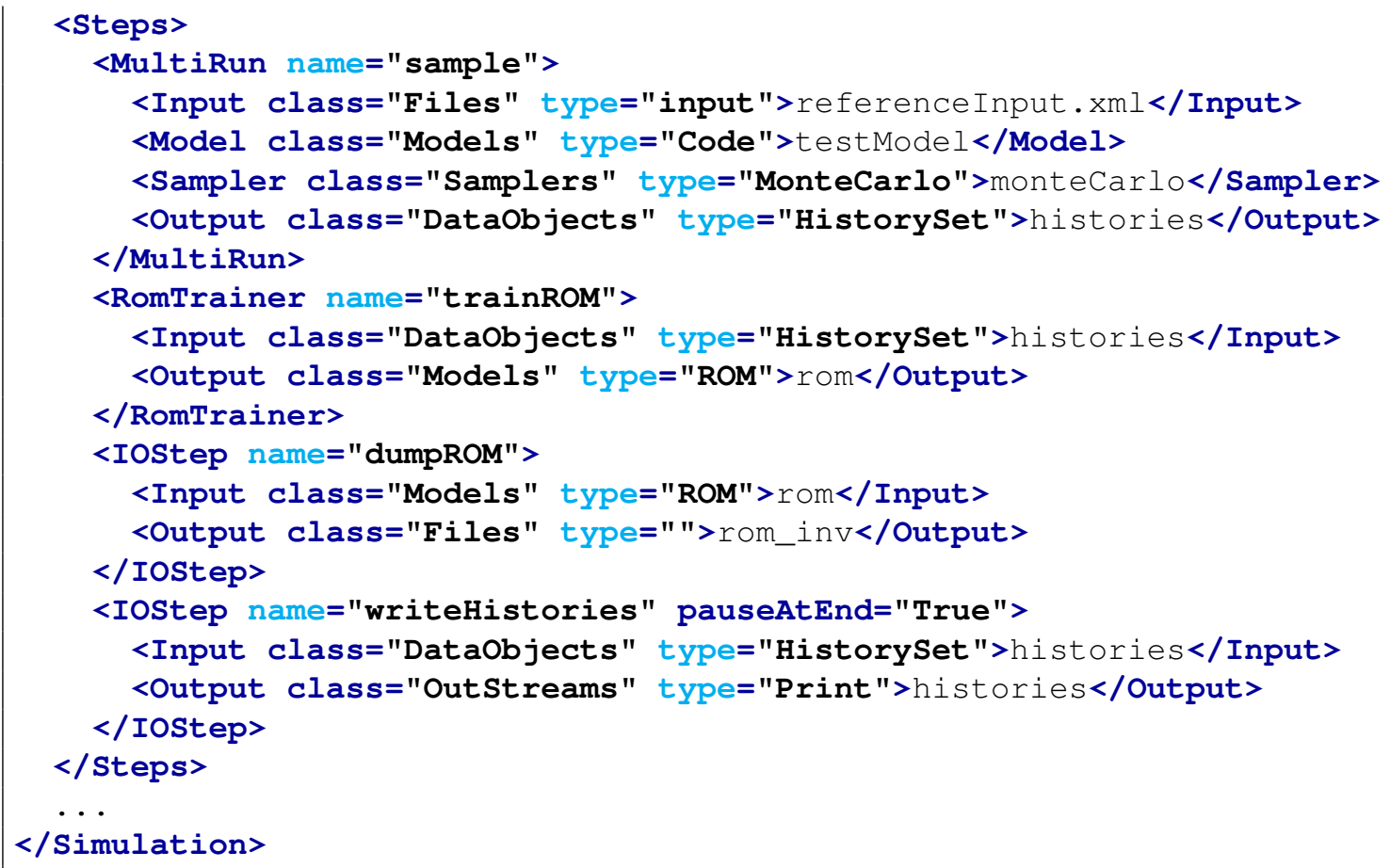

The 'HistorySet' generated by the "sample" step is used as input of the "trainROM" step. The output of the "trainROM" is "rom" which is predefined in <ROM>. If a 'PointSet' is provided as input, the output "rom" will be time-independent. The "dumpROM" step is used to serialize the "rom" to file (pickled). In this example, the file is identified in <Files $>$.

raven/tests/framework/user_guide/ravenTutorial/RomTrain.xml

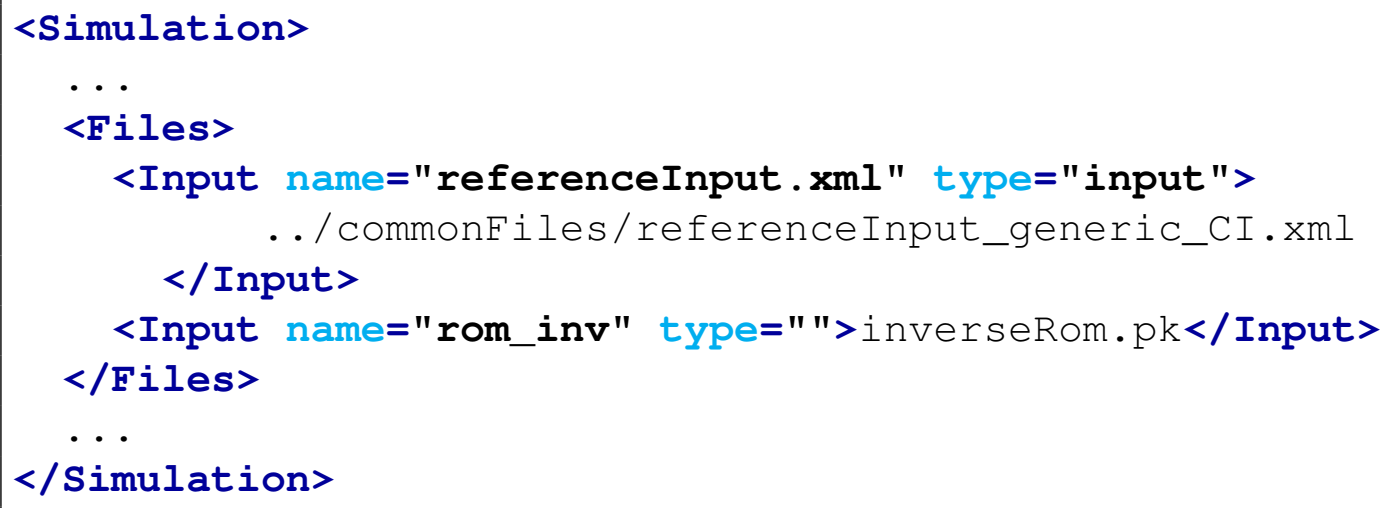

A pickled file with name "inverseRom.pk" will be generated in the working directory. This pickled ROM can be reused by RAVEN to perform additional analysis, and we will introduce this capability in the following section. Since we have four different steps to execute, the $<$ RunInfo $>$ block is modified: 


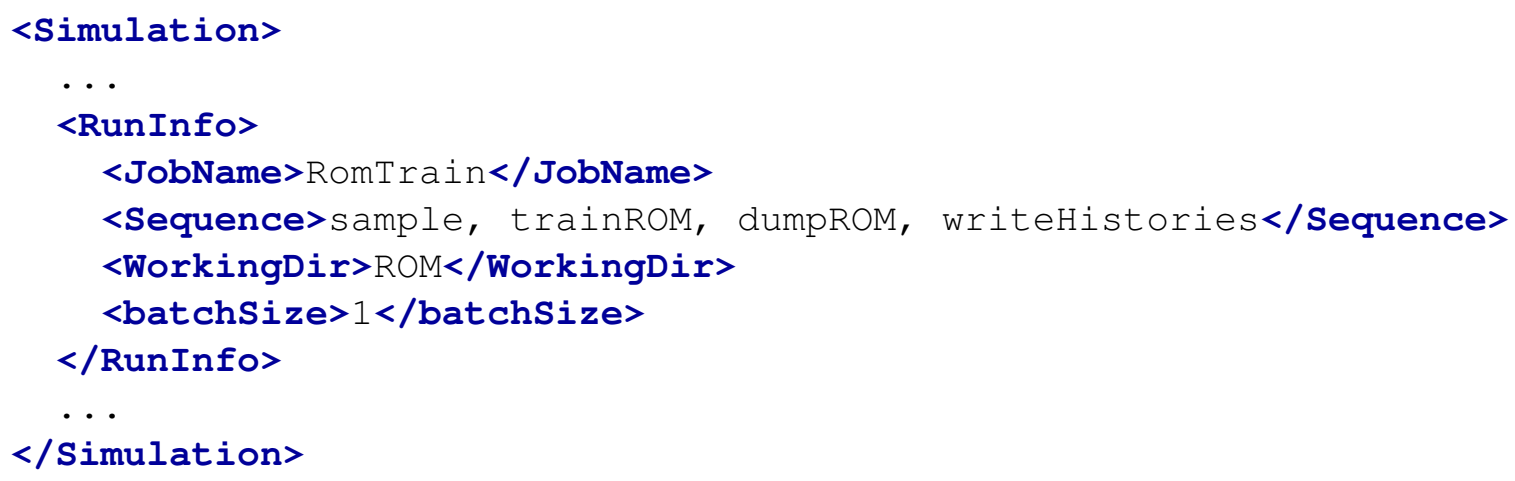

The $<$ Sequence $>$ is used to provide an ordered list of the step names that RAVEN will run.

\subsubsection{How to load and sample a ROM?}

In previous section, we have shown that RAVEN can be used to train a ROM and output the ROM to a pickled file. In this section, we will show the user how to load the pickled ROM, and how to reuse it inside RAVEN environment. In general, a $<$ ROM $>$ with subtype ' pickledROM' is used to hold the place of the ROM that will be loaded from file. The notation for this ROM is much less than a typical ROM; it only requires a name and its subtype.

Example: For this example the ROM has already been created and trained in another RAVEN run, then pickled to a file called rom_pickle.pk. In the example, the file is identified in $\langle$ Files $>$, the model is defined in <Models $>$, and the model loaded in <Steps $>$.

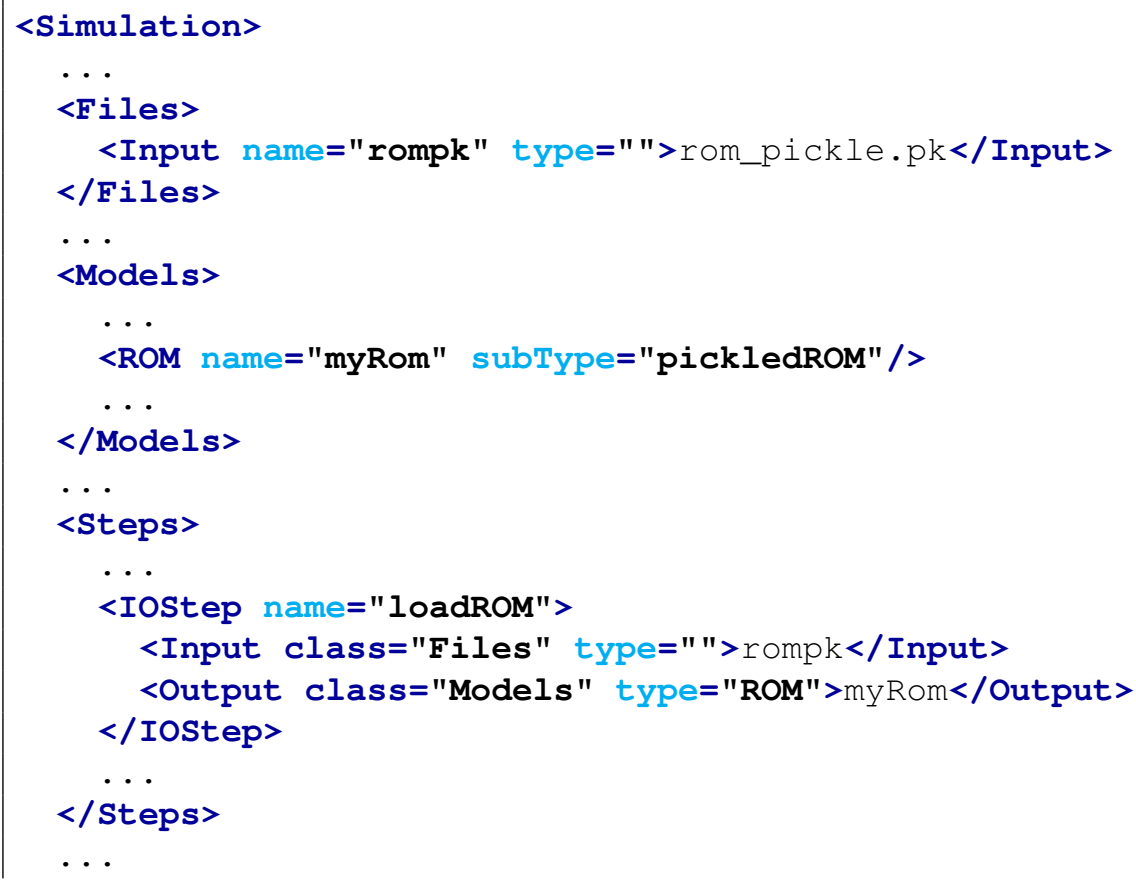


Note: When loading ROMs from file, RAVEN will not perform any checks on the expected inputs or outputs of a ROM; it is expected that a user knows at least the I/O of a ROM before trying to use it as a model. However, RAVEN does require that pickled ROMs be trained before pickling in the first place.

Initially, a pickled ROM is not usable. It can not be trained or sampled; attempting to do so will raise an error. An <IOStep> is used to load the ROM from file, at which point the ROM will have all the same characteristics as when it was pickled in a previous RAVEN run. Take AnalyticBateman for example, the pickled ROM "inverseRom.pk" is generated in previous section and copied to the commonFiles folder, RAVEN use the Files object to track the pickled ROM file.

raven/tests/framework/user_guide/ravenTutorial/RomLoad.xml

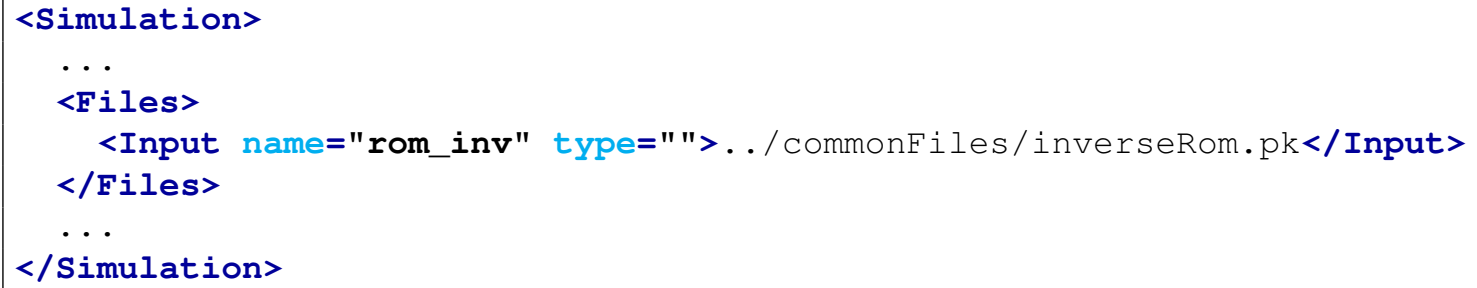

In this example, the subtype ' NDinvDistWeight' of $<$ ROM $>$ is used instead of ' pickledROM' , since the subtype of ROM is already known.

raven/tests/framework/user_guide/ravenTutorial/RomLoad.xml

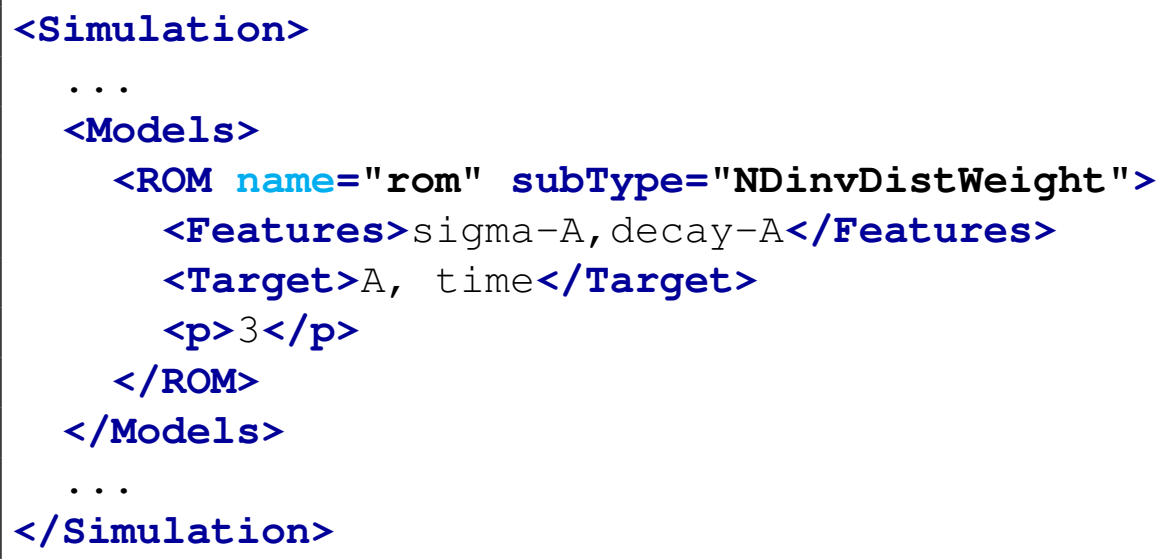

Two data objects are defined: 1) a HistorySet named "inputPlaceHolder" used as a placeholder input for the ROM sampling step, 2) a HistorySet named "histories" used to store the ROM responses from Monte Carlo samples. 
raven/tests/framework/user_guide/ravenTutorial/RomLoad.xml

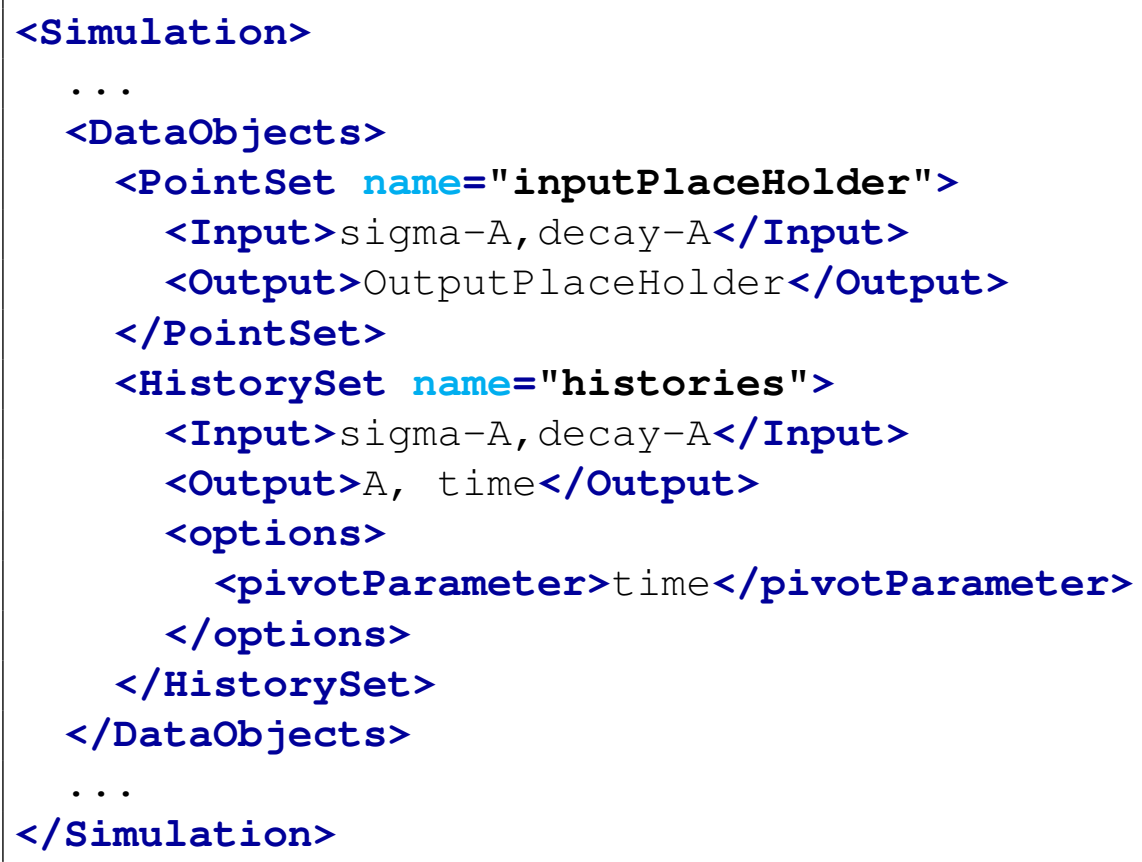

Note: In this example, a time-dependent ROM trained in previous case is used here. ' $t$ ime' is identified as Output. The sub-node <pivotParameter can be used to define the pivot variable (e.g. time) that is non-decreasing in the input HistorySet.

As mentioned before, the <IOStep> is used to load the pickled ROM. In addition, the <MultiRun> is used to sample the ROM using Monte Carlo method. Another <IOStep> is used to output the responses of ROM into a CSV file. Figure 10 shows the different evolutions of the variable $A$ for all 20 samples.

raven/tests/framework/user_guide/ravenTutorial/RomLoad.xml

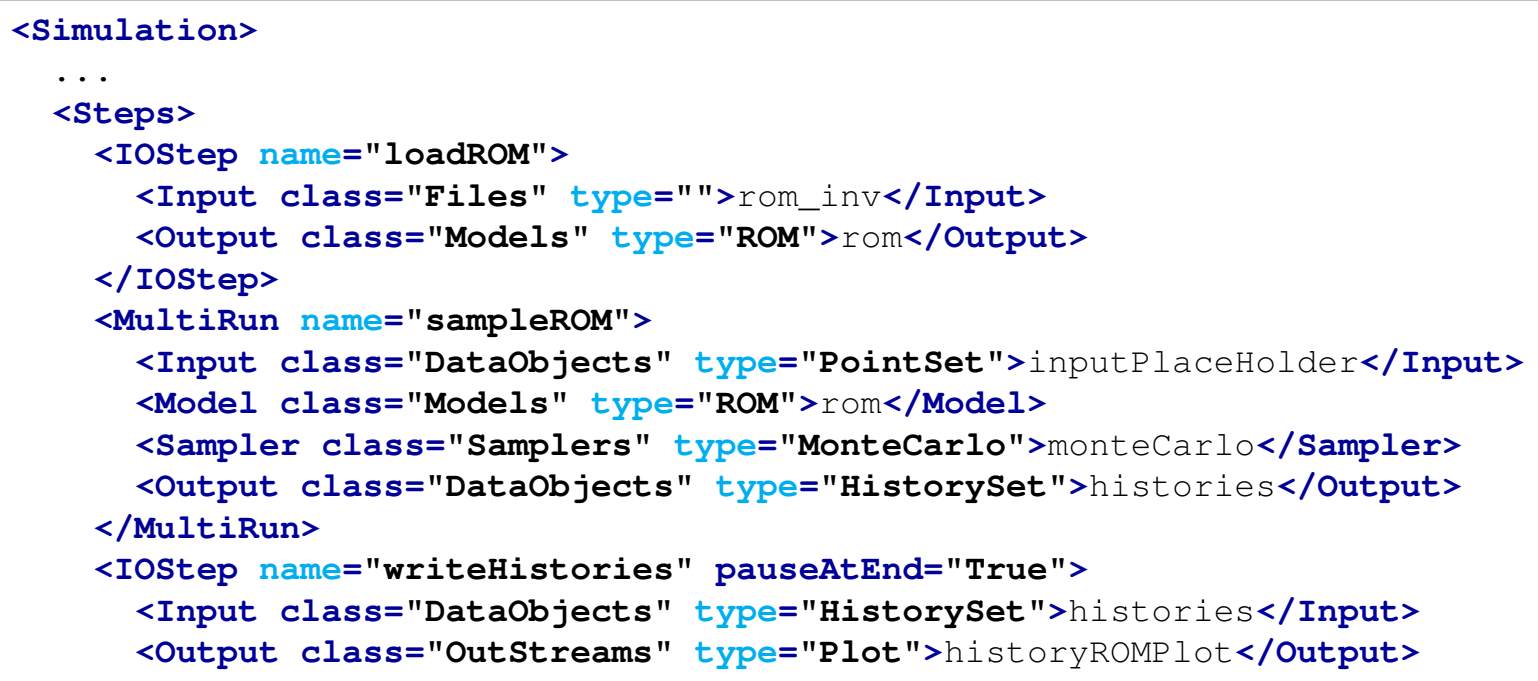




$\quad$ <Output class="OutStreams" type="Print" $>$ historiesROM</Output $>$
$</$ IOStep $>$
$</$ Steps $>$
< .

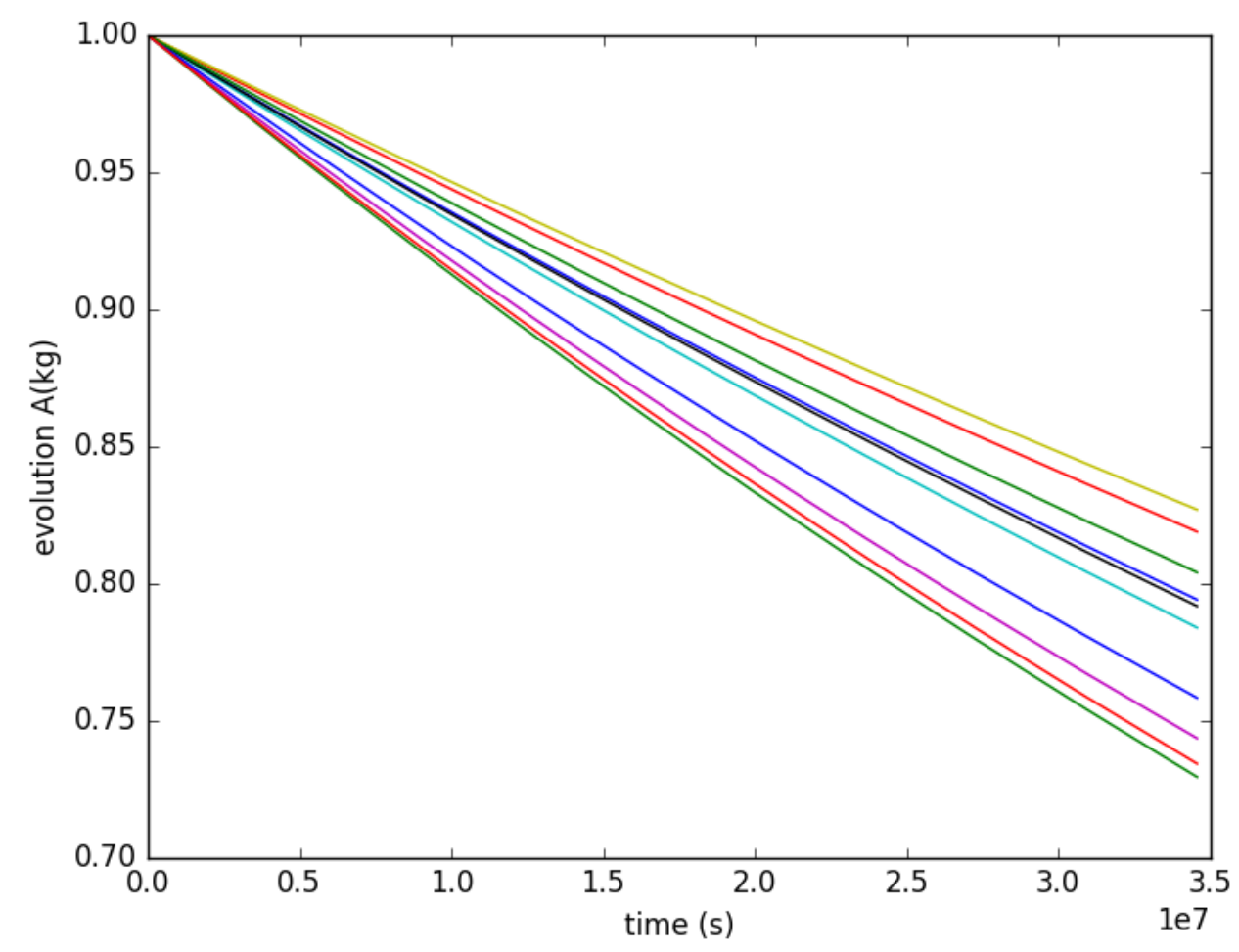

Figure 10. Plot of the histories generated by the Monte Carlo sampling of pickled ROM for variable $A$ (20 samples).

Finally, ROMs are generally not constructed for all possible inputs, geometries or representing all outputs. However, it is possible to build a ROM of faster solution with respect to the original set. The accuracy in the prediction can be obtained by further training. Figure 11 shows the general workflow for ROM construction. 


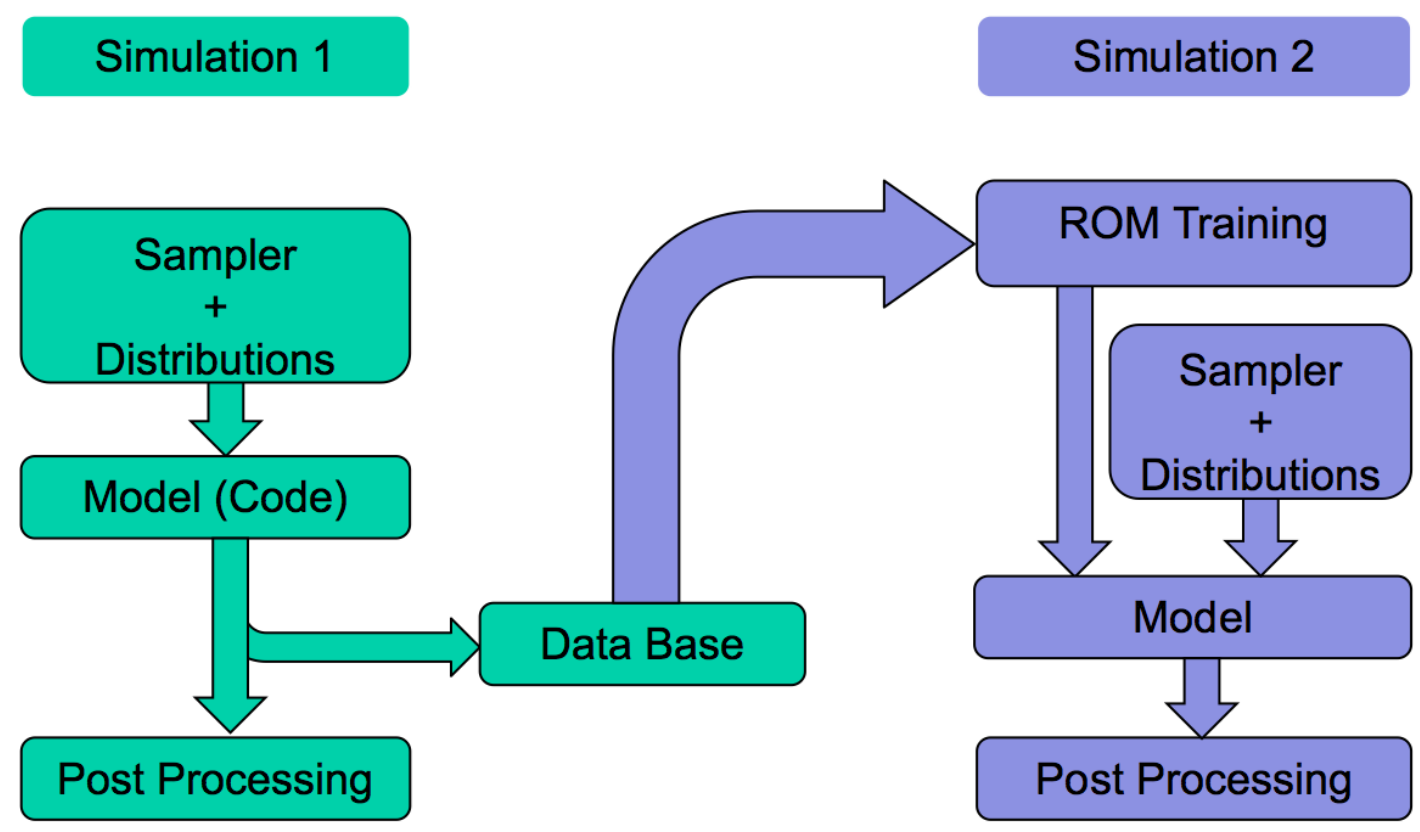

Figure 11. Workflow for ROM construction 


\subsection{Build RAVEN Input: <PostProcess>}

The PostProcess step is used to post-process data or manipulate RAVEN entities. It is intended to perform a single action that is employed by a Model of type PostProcessor. The specification of this type of step is defined within a <PostProcess $>$ XML block. As for the other objects, the attribute name is required and is used to refer to this specific entity in the <RunInfo $>$ block under the <sequence $>$ node.

In the <PostProcess> input block, the user needs to specify the objects needed for the different allowable roles. This step requires the following roles:

- <Input>: accepts Files, DataObjects or Databases.

- <Model>: Only 'Models' and 'PostProcessor' can be assigned to the node's attributes class and type, respectively.

- <Output>: accepts Files, DataObjects, Databases or OutStreams.

As mentioned before, only the model with type PostProcessor is allowed for this step. A postprocessor can be considered as an action performed on a set of data or other type of objects. Most of the post-processors contained in RAVEN, employ a mathematical operation on the data given as "Input". Currently, the following PostProcessor are available in RAVEN:

- BasicStatistics

- ComparisonStatistics

- ImportanceRank

- SafestPoint

- LimitSurface

- LimitSurfaceIntegral

- External

- TopologicalDecomposition

- RavenOutput

- DataMining

One can use the node attribute subType to select which of the post-processors to be used. As with other objects, the attribute name is always required so that other RAVEN input XML blocks can use this name to refer to this specific entity. In addition, each post-processor may require extra sub-nodes, and the user can refer to the RAVEN user manual for the detailed specifications. 
In this example, the BasicStatistics post-processor is used to demonstrate the PostProcess step. BasicStatistics is a container of the algorithms to compute many of the most important statistical quantities. Both PointSet and HistorySet can be accepted to compute the static statistics and dynamic statistics, respectively. In case an HistorySet is provided as Input, the user need to define the pivotParameter, and sometimes the user need to synchronize the HistorySet first via the Interfaced post-processor of type HistorySetSync.

raven/tests/framework/user_guide/ravenTutorial/PostProcess.xml

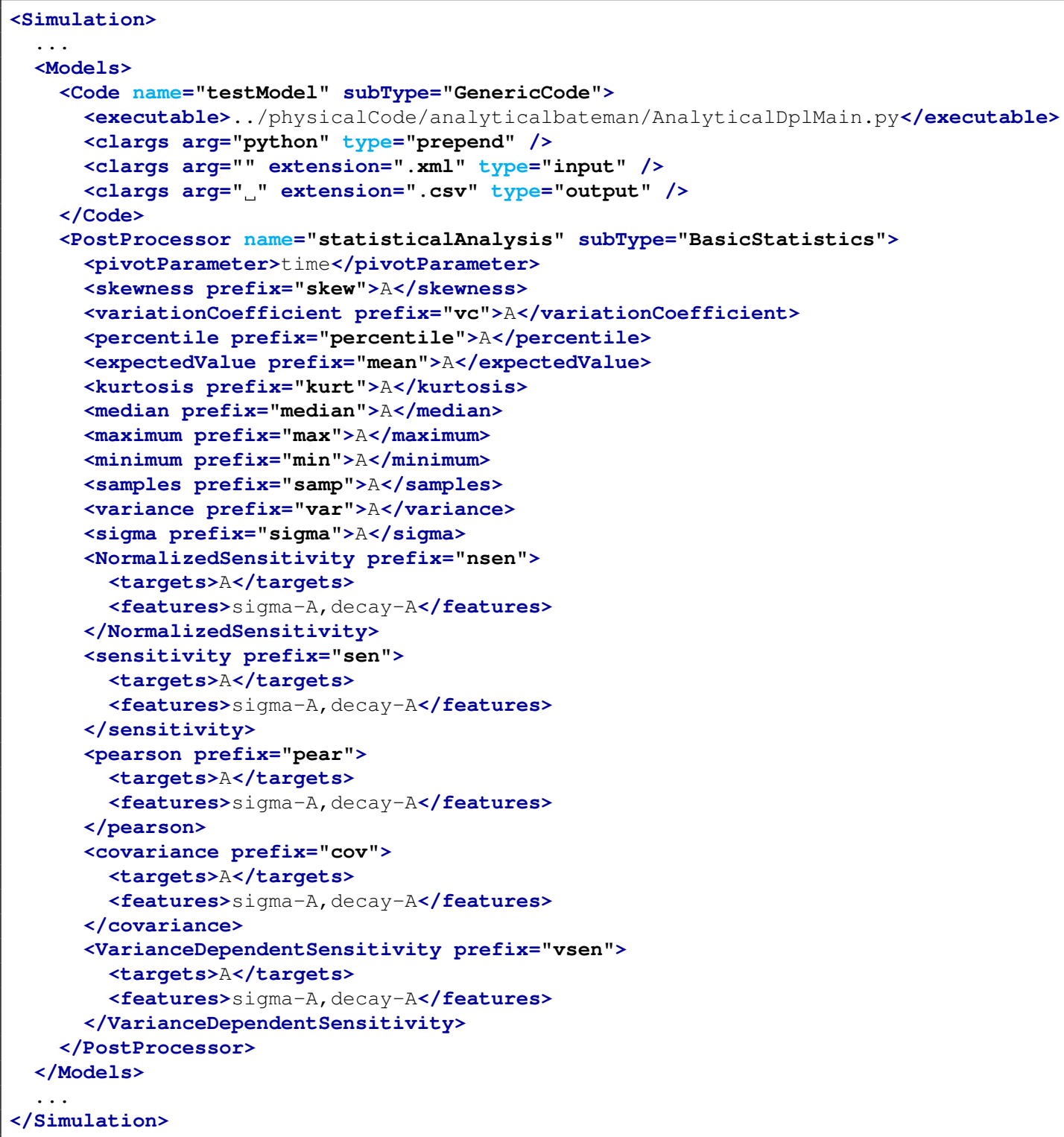

In this example, all the metrics of BasicStatistics will be computed for the response $A$.

The $<$ Files $>$ will be used to include all input and output files. In this example, a single input 
file for the driven code and two output files of the PostProcess step are defined here. As shown in the following, two output files are defined for this case study to store the static statistics and dynamic statistics information. The 'time' is used as the <pivotParameter>.

raven/tests/framework/user_guide/ravenTutorial/PostProcess.xml

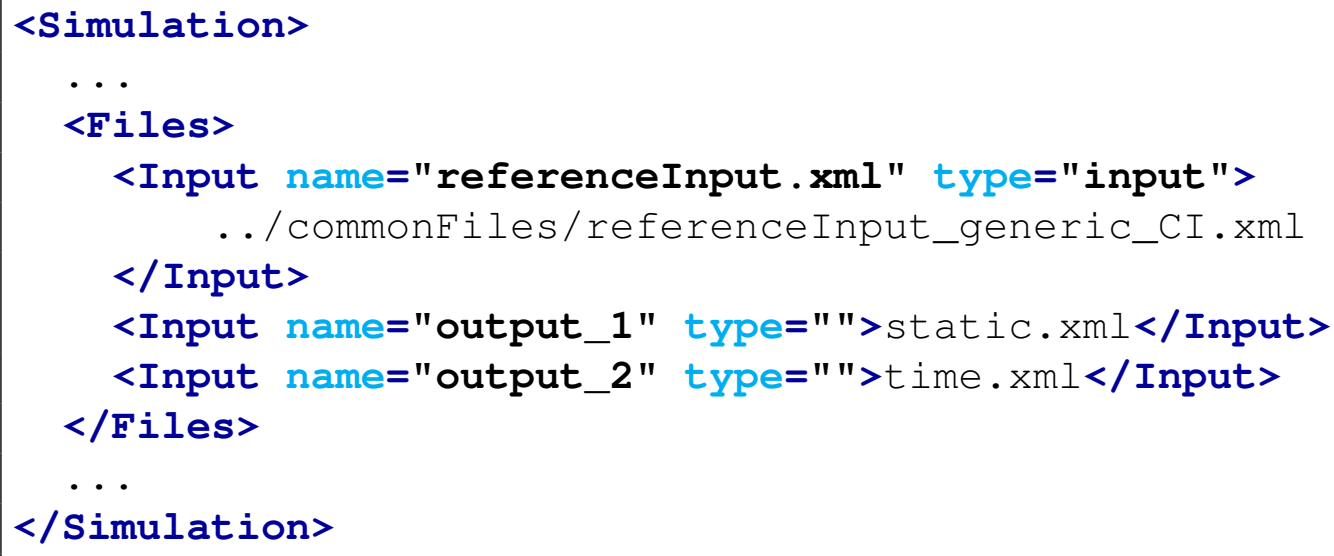

As before, all defined RAVEN entities are combined in the <Steps $>$ block.

raven/tests/framework/user_guide/ravenTutorial/PostProcess.xml

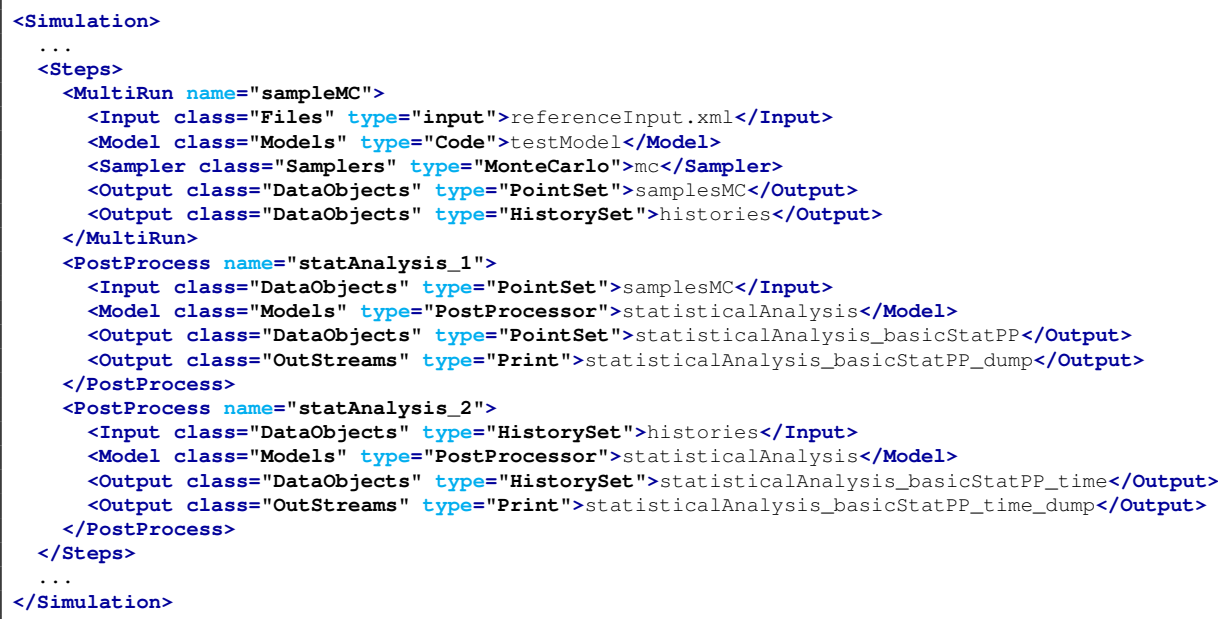

In this case, three steps have been defined:

- <MultiRun> named "sampleMC", used to run the multiple instances of the driven code and collect the outputs in the two DataObjects. As it can be seen, the <Sampler> is inputted to communicate to the Step that the driven code needs to be perturbed through the Monte Carlo sampling strategy.

- <PostProcess> named "statAnalysis_1", is used to compute all the statistical moments and FOMs based on the data obtained through the sampling strategy. As it can be noticed, 
the 'PointSet' "samplesMC" is used as input to compute the static statistics.

- <PostProcess> named "statAnalysis_2", is used to compute all the statistical moments and FOMs based on the data obtained through the sampling strategy. As it can be noticed, the 'HistorySet' "histories" is used as input to compute the dynamic statistics. 


\section{Forward Sampling Strategies}

In order to perform UQ and dynamic probabilistic risk assessment (DPRA), a sampling strategy needs to be employed. The sampling strategy perturbs the input space (domain of the uncertainties) to explore the response of a complex system in relation to selected FOMs.

The most widely used strategies to perform UQ and PRA are generally collected in RAVEN as Forward samplers. Forward samplers include all the strategies that simply perform the sampling of the input space. These strategies sample without exploiting, through learning approaches, the information made available from the outcomes of evaluation previously performed (adaptive sampling) and the common system evolution (patterns) that different sampled calculations can generate in the phase space (Dynamic Event Tree).

As mentioned in Section ??, RAVEN has several different Forward samplers:

- Monte-Carlo

- Grid-based

- Stratified and its specialization named Latin Hyper Cube.

In addition, RAVEN posses advanced Forward sampling strategies that:

- Build a grid in the input space selecting evaluation points based on characteristic quadratures as part of stochastic collocation for generalized polynomial chaos method (Sparse Grid Collocation sampler);

- Use high-density model reduction (HDMR) a.k.a. Sobol decomposition to approximate a function as the sum of interactions with increasing complexity (Sobol sampler).

In the following subsections, we provide examples of input files in RAVEN using the method, with explanatory commentary.

\subsection{Monte-Carlo sampling through RAVEN}

The Monte-Carlo method is one of the most-used methodologies in several mathematic disciplines. In this section, we will explain the techniques for employing this methodology in RAVEN, and we recommend the user to read the theory manual to explore the theory of the method. The goals of this section are about learning how to:

1. Set up a simple Monte-Carlo sampling for perturbing the input space of a driven code 
2. Load the outputs of the code into RAVEN DataObjects (HistorySets and PointSets)

3. Print the contents of DataObjects to file

4. Generate plots of the sampling results.

In order to accomplish these tasks, the following RAVEN Entities (XML blocks in the RAVEN input file) are needed:

\section{RunInfo:}

raven/tests/framework/user_guide/ForwardSamplingStrategies/forwardSamplingMontecarlo.xml

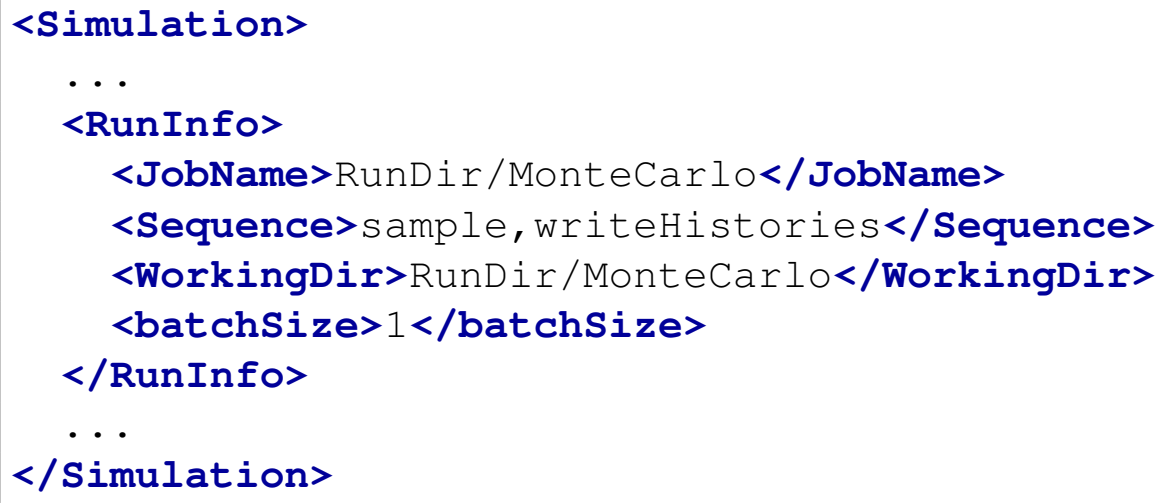

As discussed in Section ??, the RunInfo Entity sets up the analysis that the user wants to perform. In this specific case, two steps (<Sequence>) are sequentially run using 12 processors (<batchsize>). This means that 12 instances of the driven code are run simultaneously. Every time a simulation ends, a new one is launched. Note that the $<$ JobName $>$ is not required, but is useful in identifying the input file.

\section{Files:}

raven/tests/framework/user_guide/ForwardSamplingStrategies/forwardSamplingMontecarlo.xml

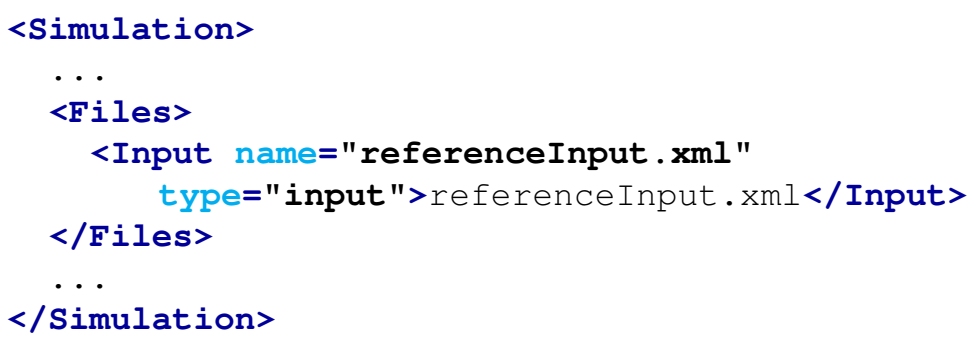

Since the driven code uses a single input file, in this section the original input is identified and named. As detailed in the user manual, the attribute name represents the alias that is used in all the other input blocks in order to refer to this file. 


\section{Models:}

raven/tests/framework/user_guide/ForwardSamplingStrategies/forwardSamplingMontecarlo.xml

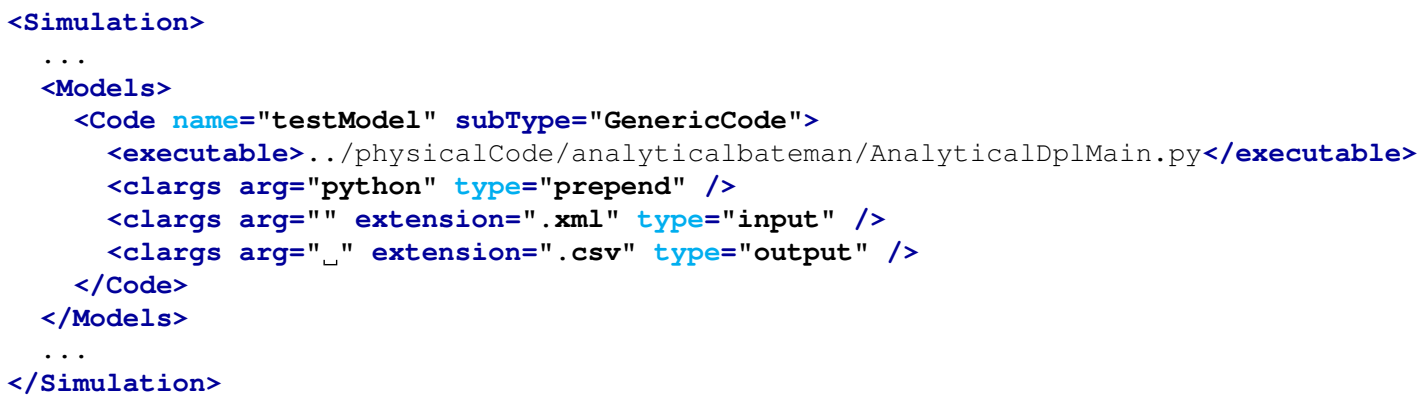

The Model used in this example is the AnalyticalBateman external model, which is defined in section 2.1. This model uses a keyword-based input file and exports its output file in a CSV format, so the Generic Code interface is used.

\section{Distributions:}

raven/tests/framework/user_guide/ForwardSamplingStrategies/forwardSamplingMontecarlo.xml

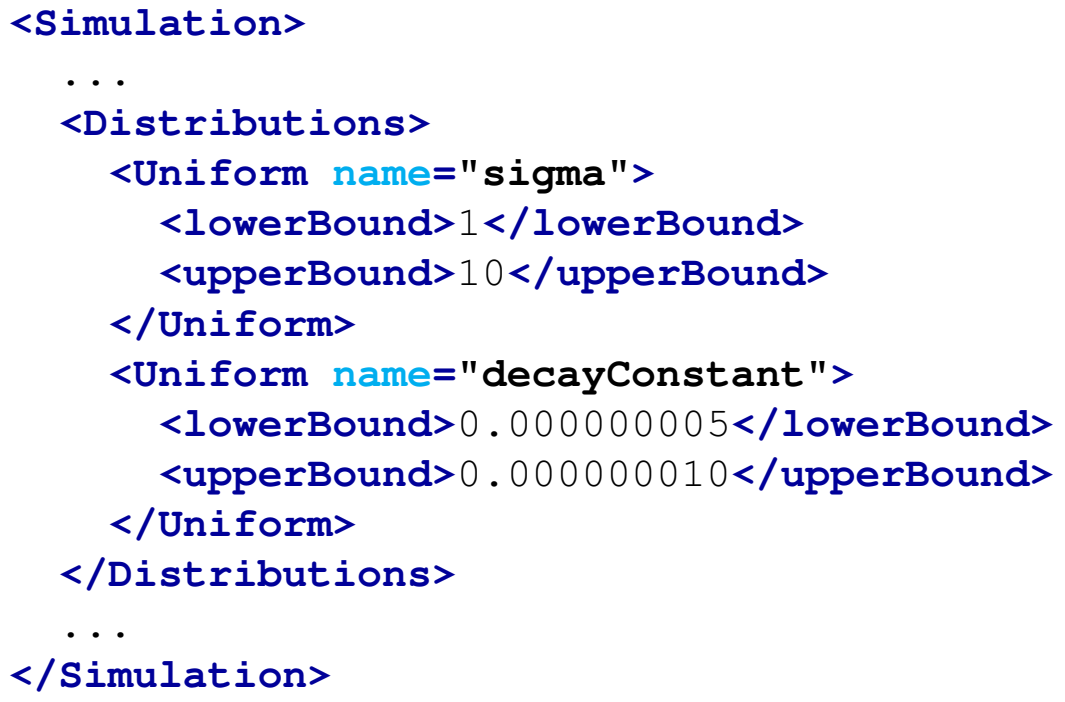

In the <Distributions $>$ block, the stochastic model for the uncertainties treated by the $<$ Sampler $>$ is defined. In this case two distributions are defined:

- sigma $\sim \mathbb{U}(1,10)$, used to model the uncertainties associated with the sigma Model variables

- decayConstant $\sim \mathbb{U}(0.5 e-8,1 e-8)$, used to model the uncertainties associated with the Model variable decay constants. Note that the same distribution can be re-used for multiple input variables, while still keeping those variables independent.

\section{Samplers:}

raven/tests/framework/user_guide/ForwardSamplingStrategies/forwardSamplingMontecarlo.xml 


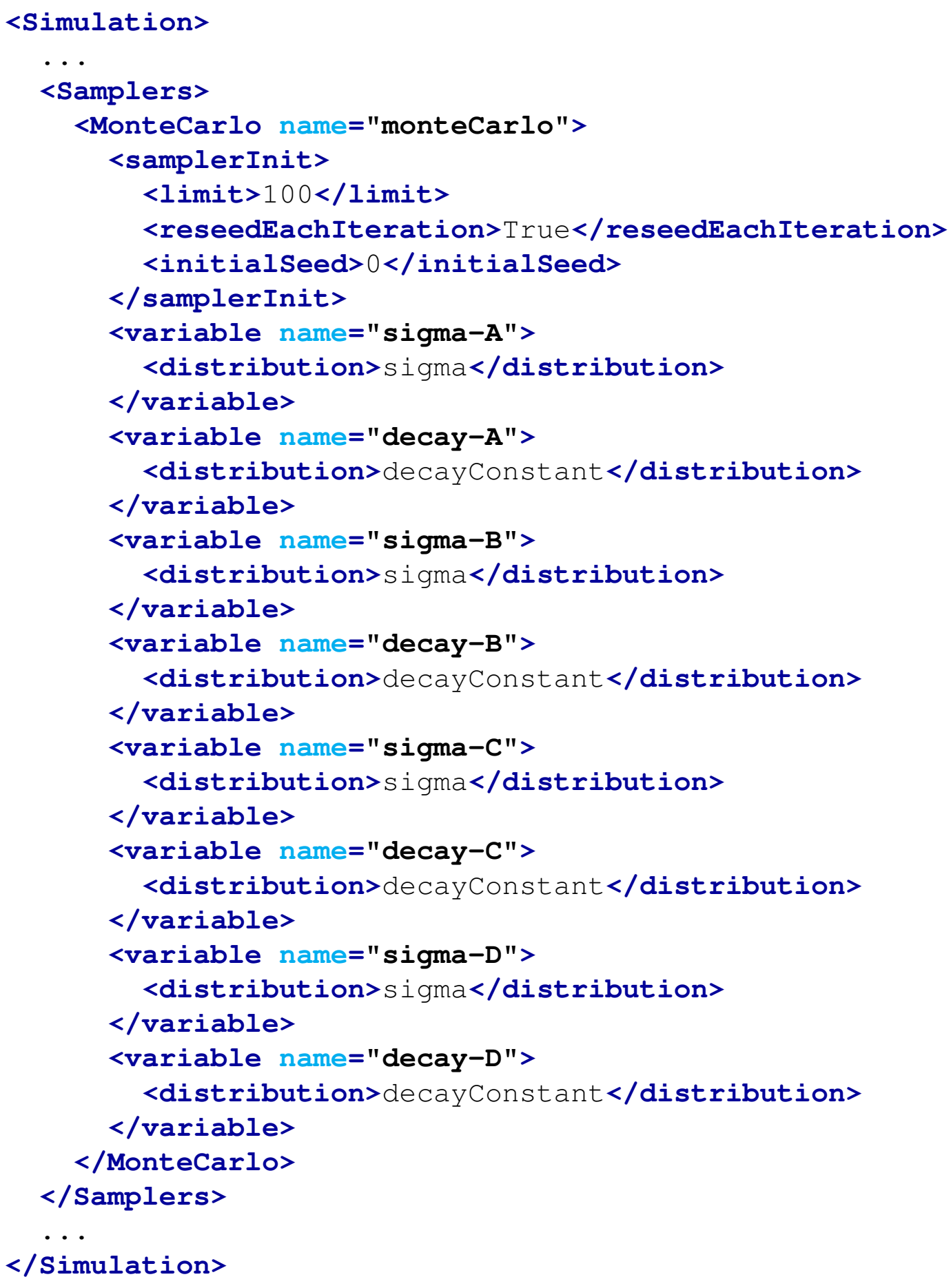

To employ the Monte-Carlo sampling strategy (100 samples), a <MonteCarlo> node needs to be defined. The Monte-Carlo method is employed on eight model variables, each of which are listed by name and are associated with a distribution. Note that all the decayand sigma- variables are associated with the distributions decayConstant and sigma, re- 
spectively.

\section{DataObjects:}

raven/tests/framework/user_guide/ForwardSamplingStrategies/forwardSamplingMontecarlo.xml

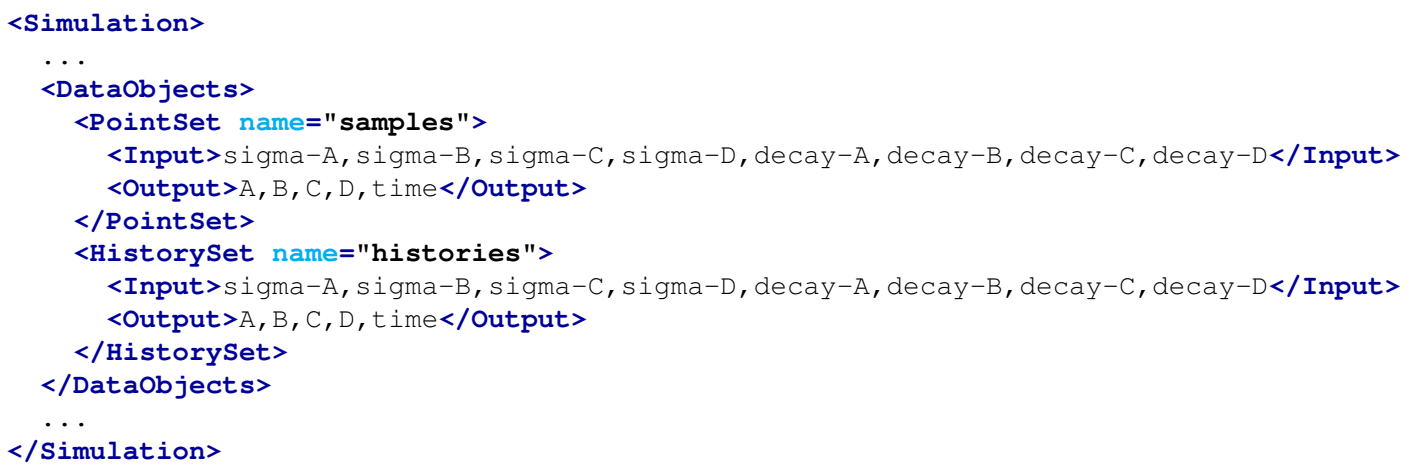

In this block, two DataObjects are defined to store results: 1) a PointSet named "samples", 2) a HistorySet named "histories". Note that in the <Input> node all the uncertainties perturbed through the Monte-Carlo strategy are listed. By this, any realization in the input space is linked in the DataObject to the outputs listed in the <Output> node.

\section{OutStreams:}

raven/tests/framework/user_guide/ForwardSamplingStrategies/forwardSamplingMontecarlo.xml

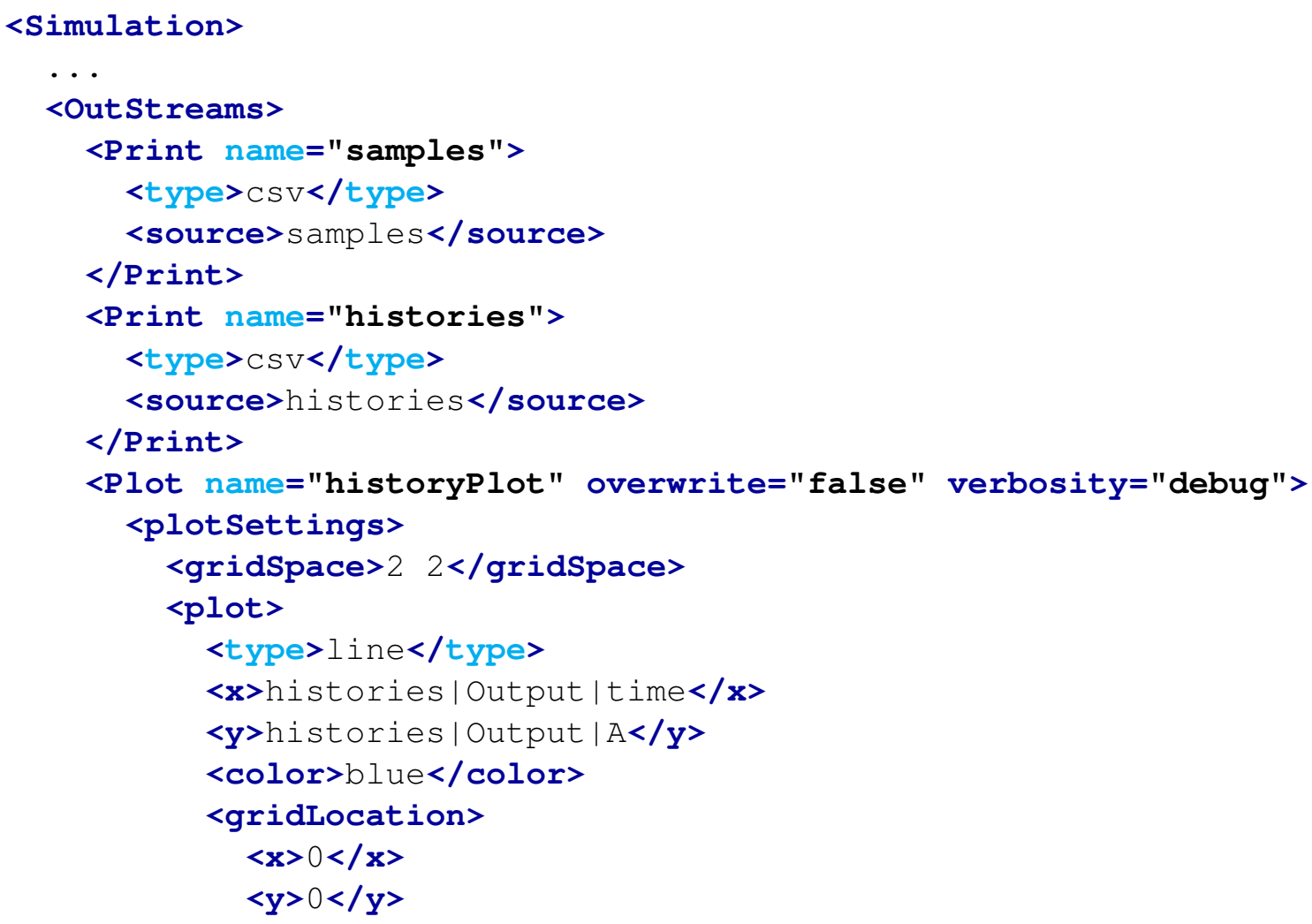




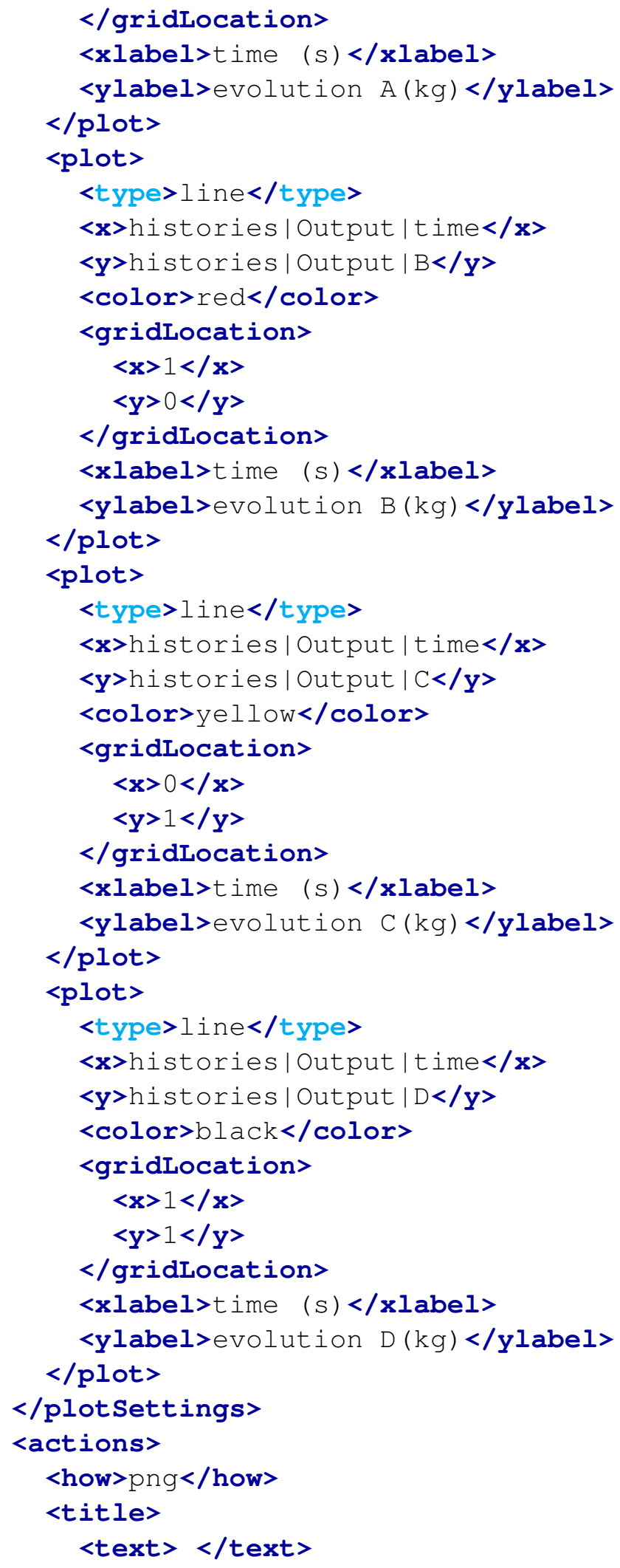




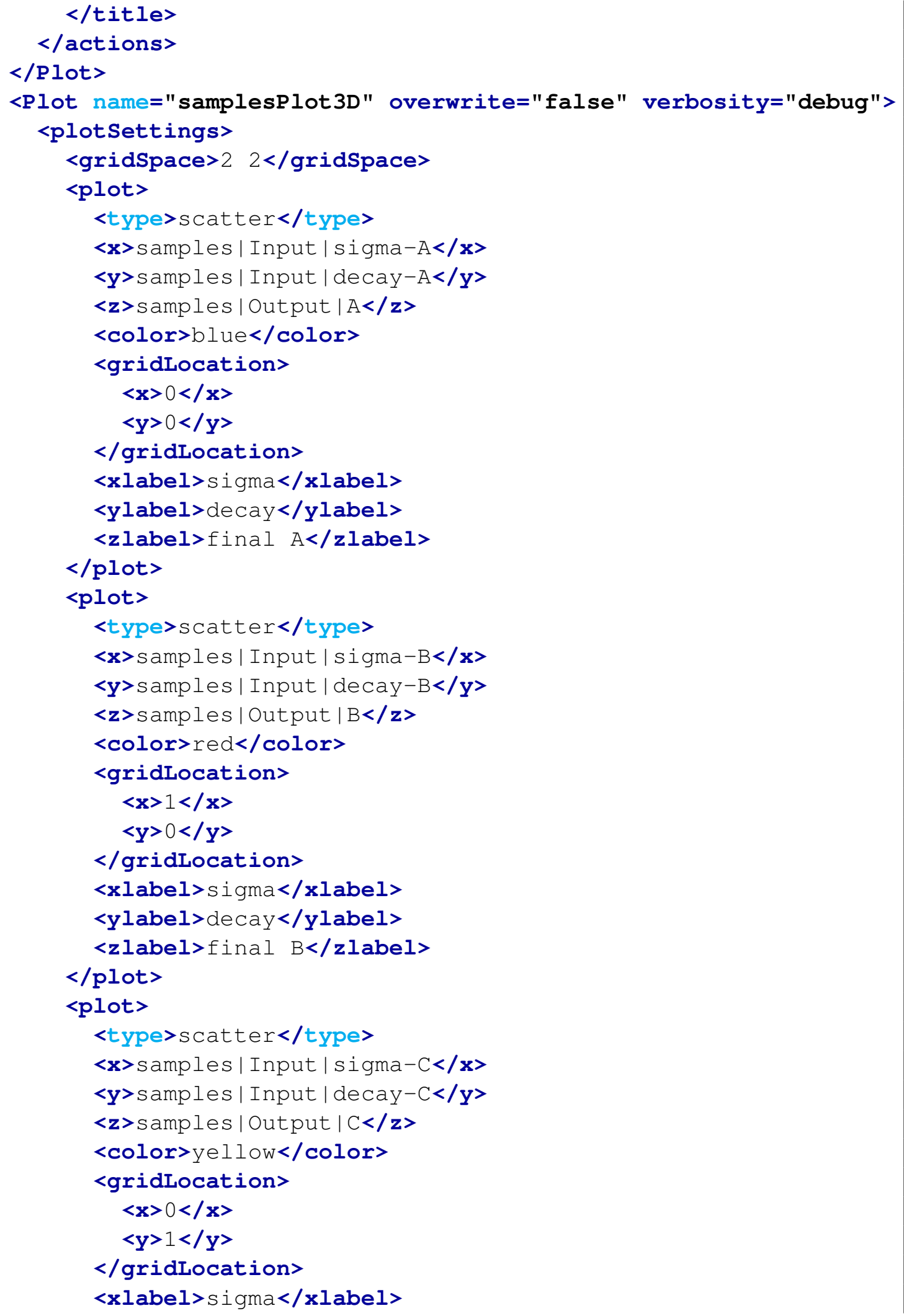




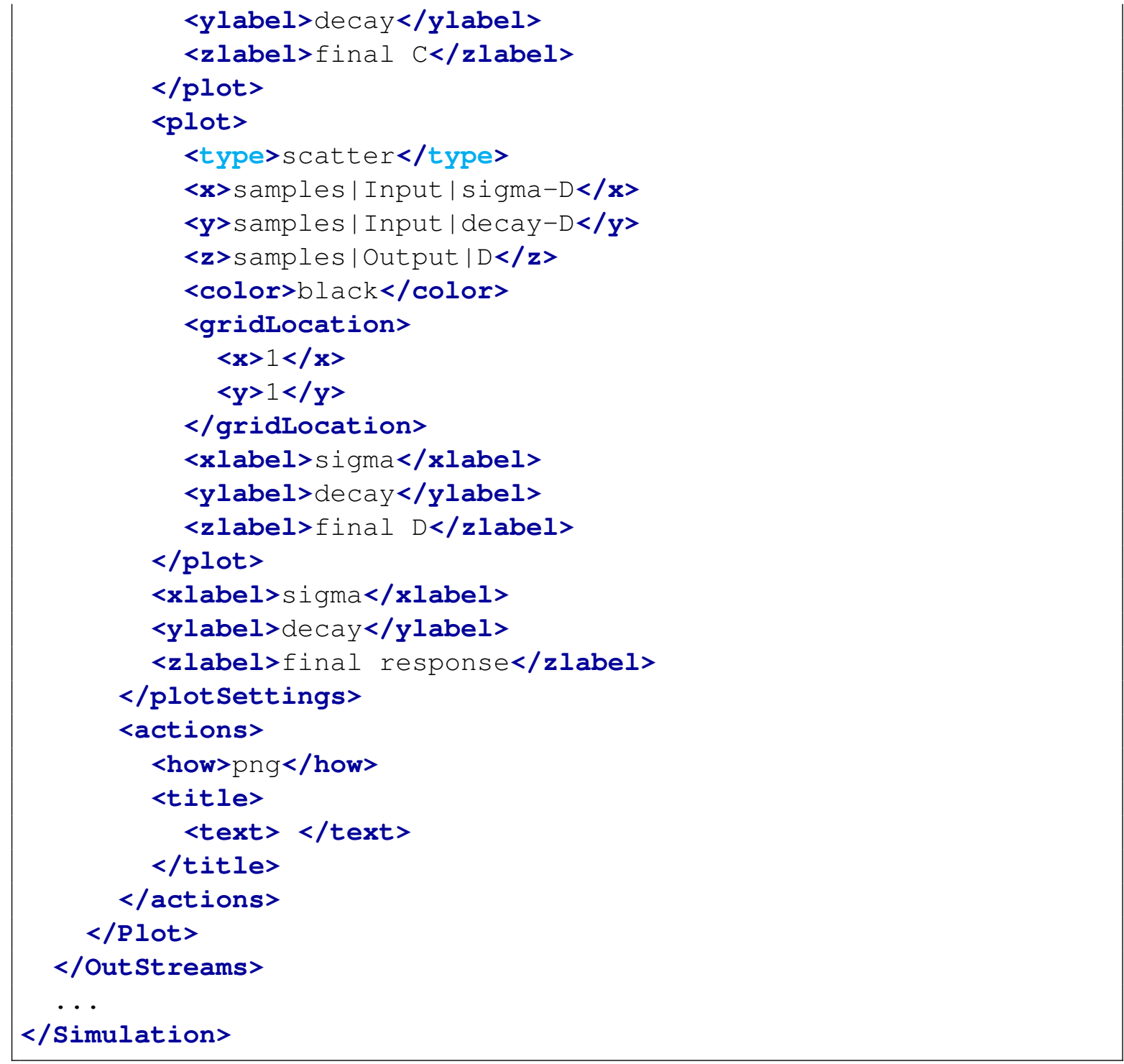

To see the results of the simulation, <OutStreams> are included in the input. In this block, both OutStream types are used:

- Print:

- "samples" connected with the DataObjects Entity "samples" (<source>)

- "histories" connected with the DataObjects Entity "histories" (<source>)

Note that in RAVEN, multiple entities can have the same name, as it takes a class, a type, and a name to uniquely identify a RAVEN object. When the two OutStream objects are used, all the information contained in the linked DataObjects are going to be exported in CSV files (<type $>$ ).

- Plot: 

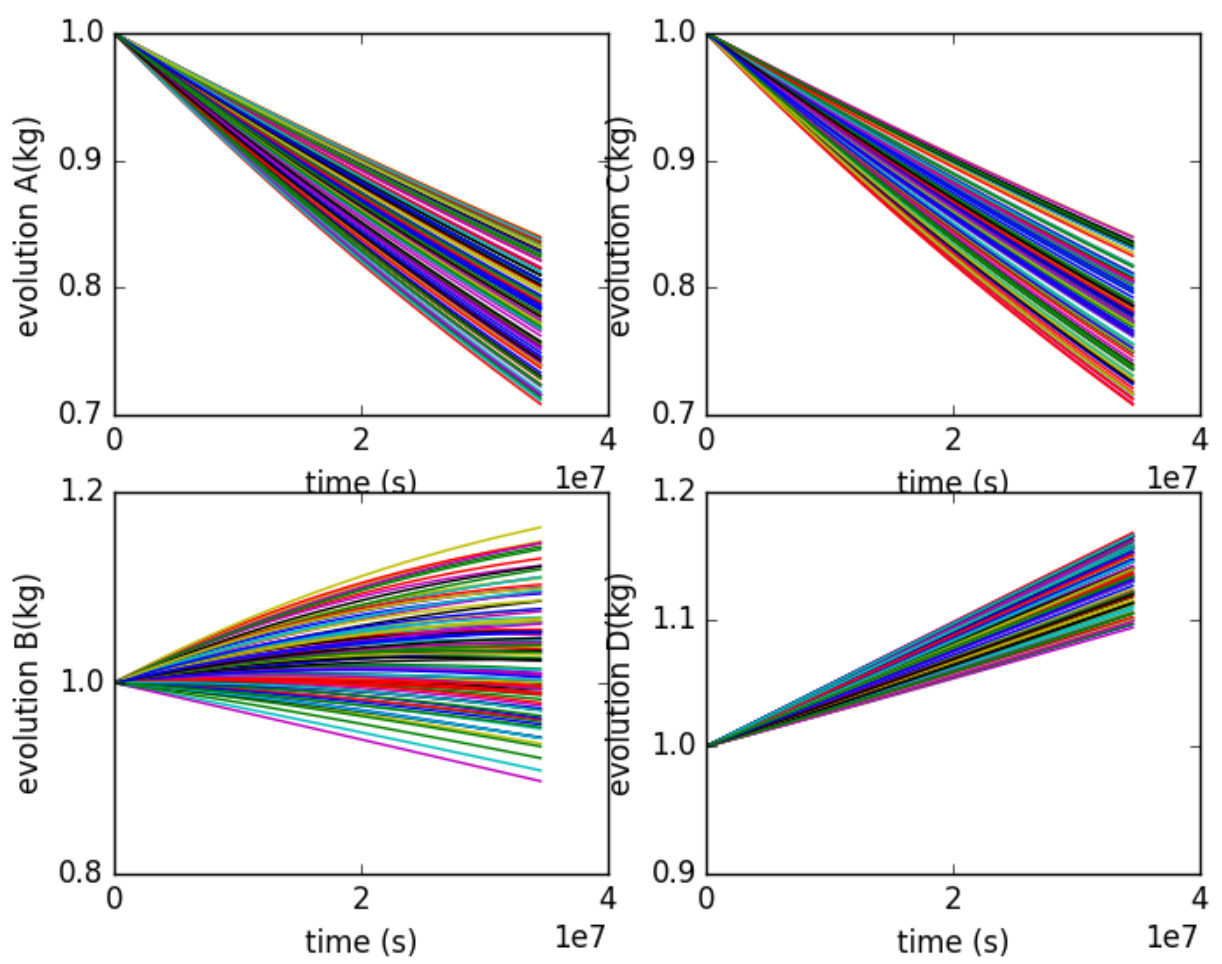

Figure 12. Plot of the histories generated by the Monte Carlo sampling for variables $A, B, C, D$.

- "historiesPlot" connected with the DataObjects Entity "samples". This plot will draw the final state of the variables $A, B, C, D$ with respect to the input variables sigma(s) and decay(s).

_ "samplesPlot3D" connected with the DataObjects Entity "histories". This plot will draw the evolution of the variables $A, B, C, D$.

Note that both plots use gridded subplots. Four plots are placed in each of the figures.

\section{Steps:}

raven/tests/framework/user_guide/ForwardSamplingStrategies/forwardSamplingMontecarlo.xml

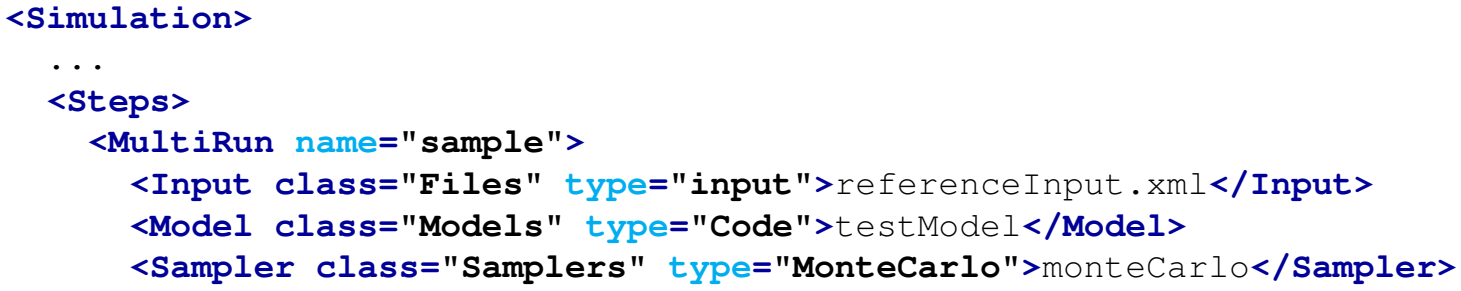



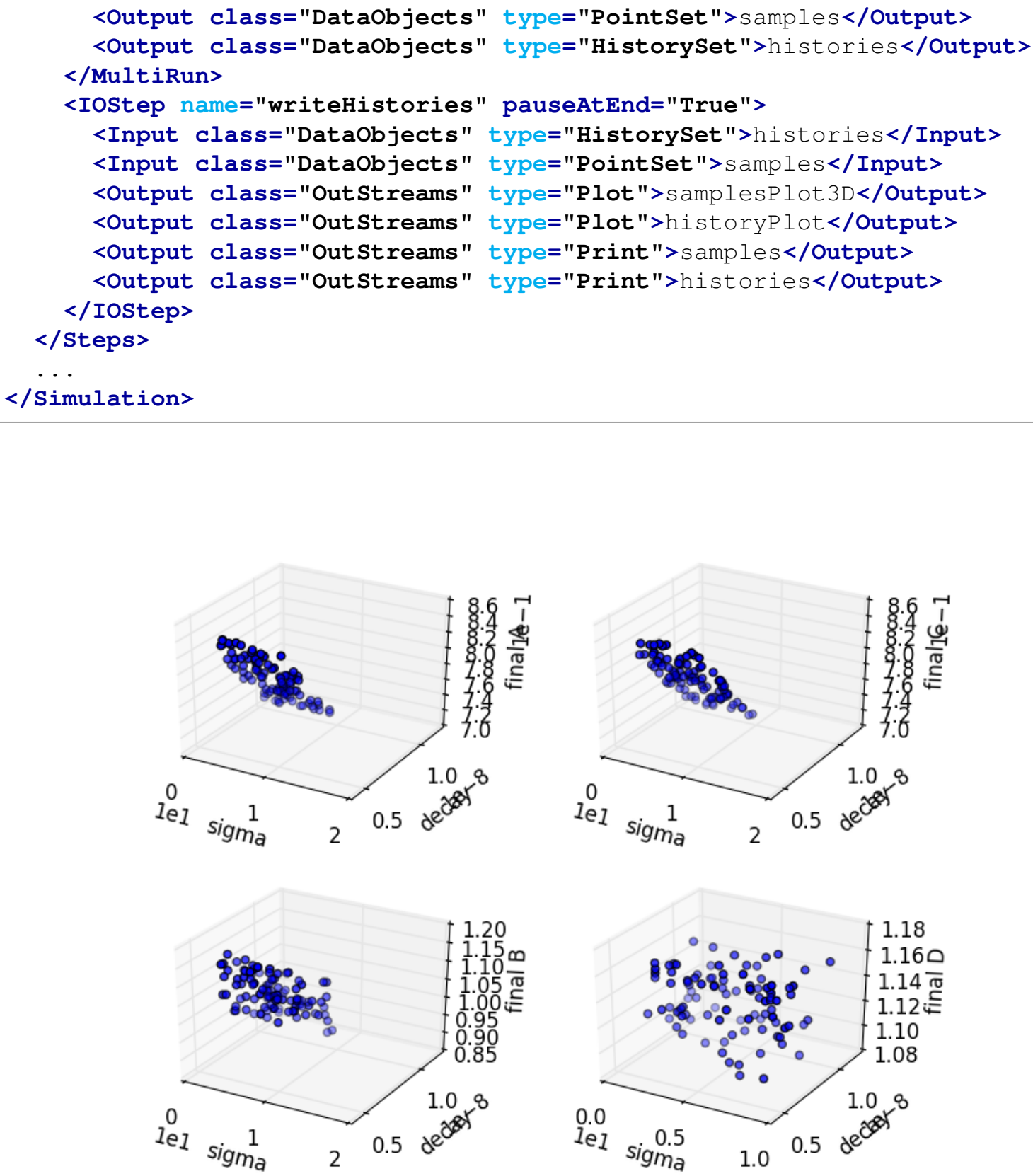

Figure 13. Plot of the samples generated by the MC sampling for variables $A, B, C, D$.

Once all the other entities are defined in the RAVEN input file, they must be combined in the <Steps> block, which dictates the workflow of RAVEN. For this case, two <Steps $>$ are defined: 
- <MultiRun> "sample", used to run the multiple instances of the driven code and collect the outputs in the two DataObjects. As it can be seen, the <Sampler> is specified to communicate to the Step that the driven code needs to be perturbed through the Monte-Carlo sampling.

- <IOStep> named "writeHistories", used to 1) dump the "histories" and "samples" DataObjects Entity to a CSV file and 2) plot the data in the EPS file.

Figures 12 and 13 show the report generated by RAVEN of the evolution of the variables $A, B, C, D$ and their final values, respectively.

\subsection{Grid sampling through RAVEN}

The Grid sampling method (also known as Full Factorial Design of Experiment) represents one of the simplest methodologies that can be employed in order to explore the interaction of multiple random variables with respect selected FOMs. The goal of this section is to show how to:

1. Set up a simple Grid sampling for performing a parametric analysis of a driven code

2. Load the outputs of the code into the RAVEN DataObjects system

3. Print out what contained in the DataObjects

4. Generate basic plots of the code result.

In order to accomplish these tasks, the following RAVEN Entities (XML blocks in the input files) are required:

\section{RunInfo:}

raven/tests/framework/user_guide/ForwardSamplingStrategies/forwardSamplingGrid.xml

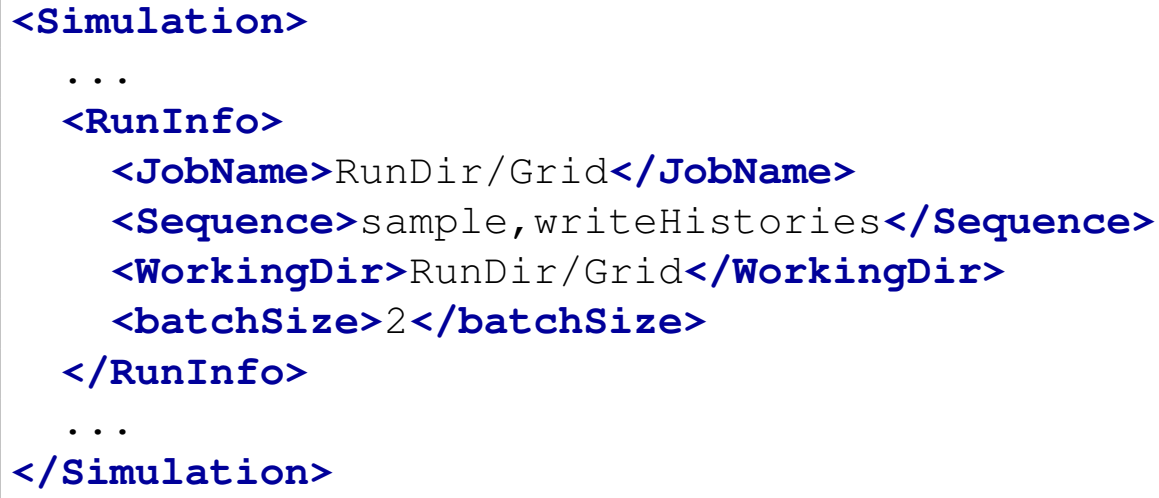


As shown in Section ??, the RunInfo Entity is intended to set up the desired analysis. In this specific case, two steps (<Sequence>) are sequentially run using 12 processors (<batchSize $>$ ). This means that 12 instances of the driven code are run simultaneously. Every time a simulation ends, a new one is launched.

\section{Files:}

raven/tests/framework/user_guide/ForwardSamplingStrategies/forwardSamplingGrid.xml

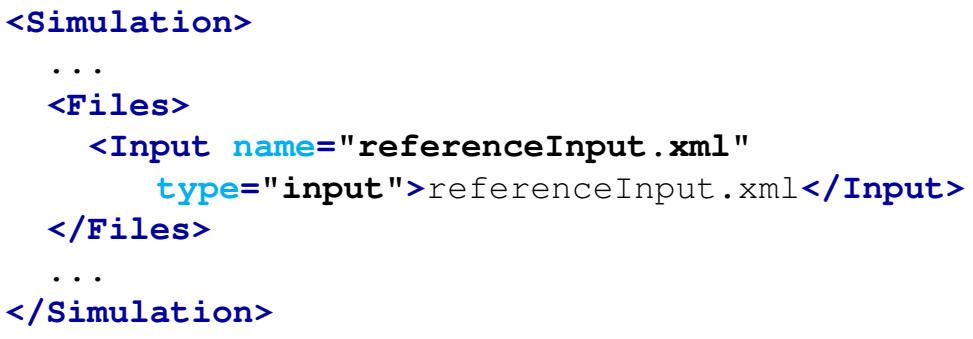

Since the driven code uses a single input file, in this section the original input is placed. As described in the user manual [4] the attribute name represents the alias that is used in all the other input blocks in order to refer to this file.

\section{Models:}

raven/tests/framework/user_guide/ForwardSamplingStrategies/forwardSamplingGrid.xml

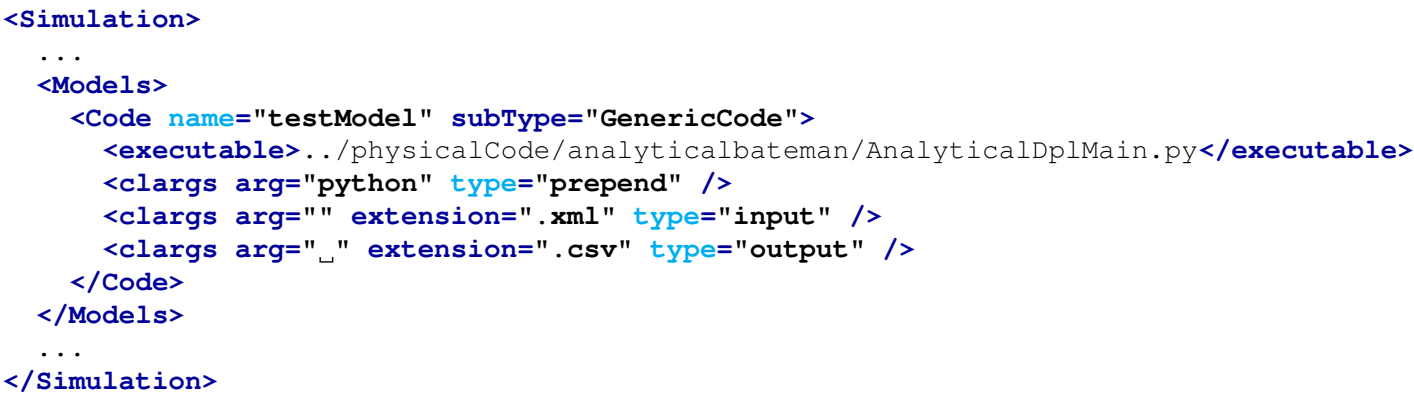

The Model here is represented by the AnalyticalBateman, which already dumps its output file in a CSV format (standard format that RAVEN can read). For this reason, the GenericCode interface is used.

\section{Distributions:}

raven/tests/framework/user_guide/ForwardSamplingStrategies/forwardSamplingGrid.xml

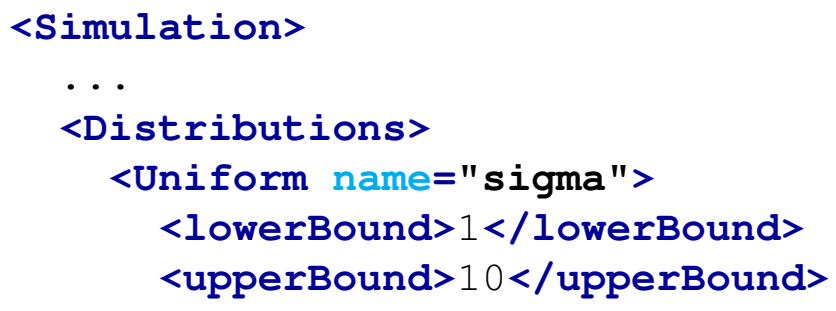




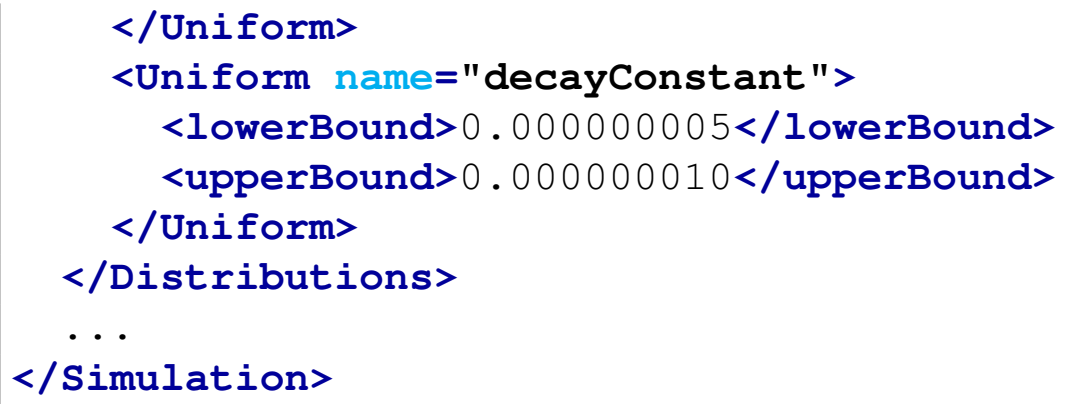

In the Distributions XML section, the stochastic model for the uncertainties treated by the Grid sampling are reported. In this case two distributions are defined:

- sigma $\sim \mathbb{U}(1,10)$, used to model the uncertainties associated with the Model sigma(s)

- decayConstant $\sim \mathbb{U}(0.5 e-8,1 e-8)$, used to model the uncertainties associated with the Model decay constants.

\section{Samplers:}

raven/tests/framework/user_guide/ForwardSamplingStrategies/forwardSamplingGrid.xml

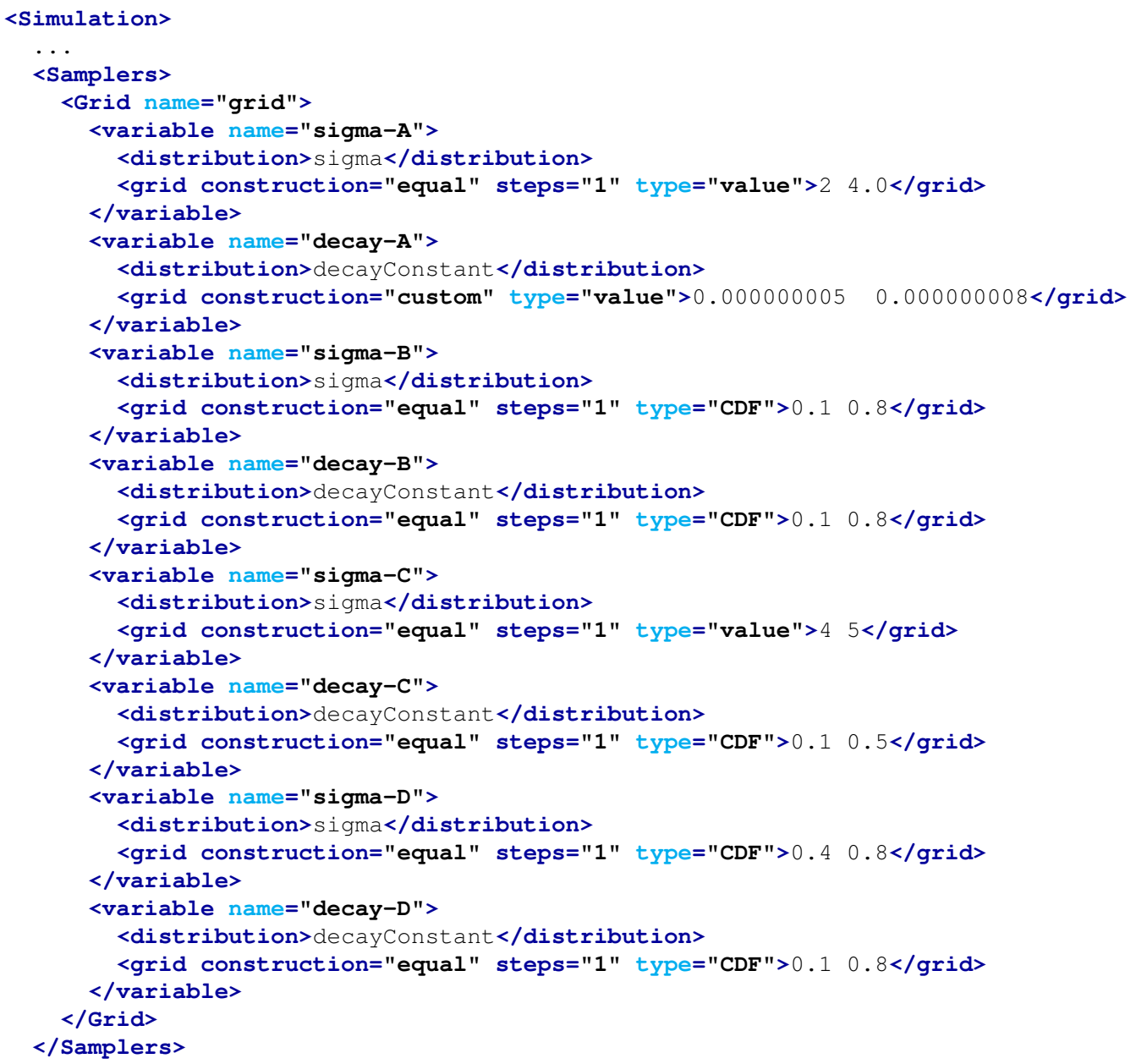


...

$</$ Simulation $>$

To employ the Grid sampling strategy, a <Grid $>$ node needs to be specified. As shown above, in each variable section, the $\langle$ grid $\rangle$ is defined. The number of samples finally requested are equal to $n_{\text {samples }}=\prod_{i=1}^{n} n_{\text {steps }_{i}+1}=256$. Note that, for each variable, can be defined either in probability (CDF) or in absolute value.

\section{DataObjects:}

raven/tests/framework/user_guide/ForwardSamplingStrategies/forwardSamplingGrid.xml

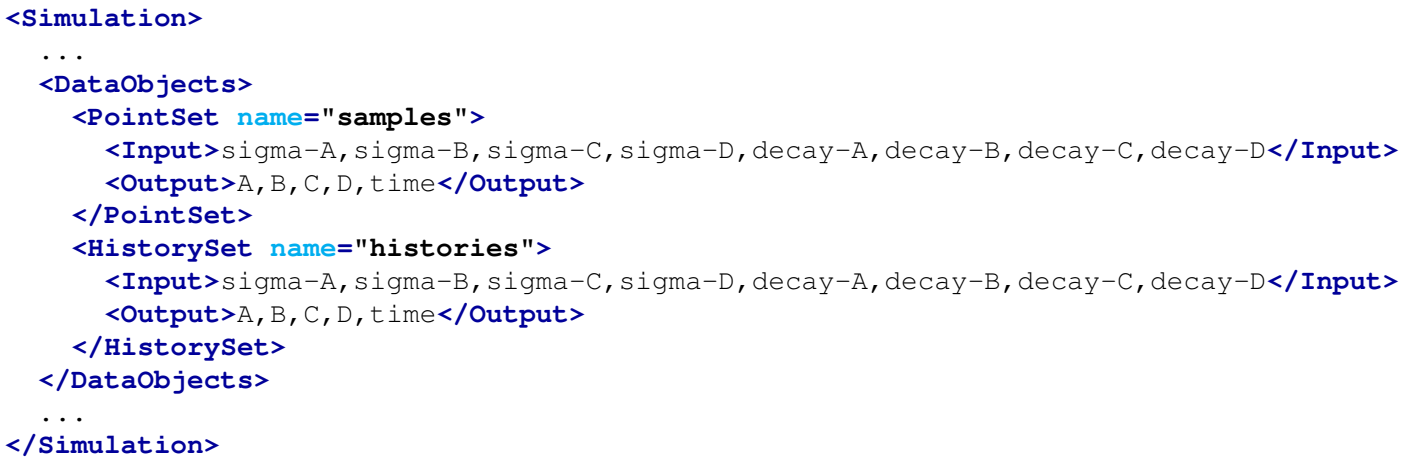

Int this block, two DataObjects are defined: 1) PointSet named "samples", and 2) HistorySet named "histories". In the <Input> node all the variables perturbed through the Grid strategy are listed. In this way, any realization in the input space is linked to the outputs listed in the <output> node.

\section{OutStreams:}

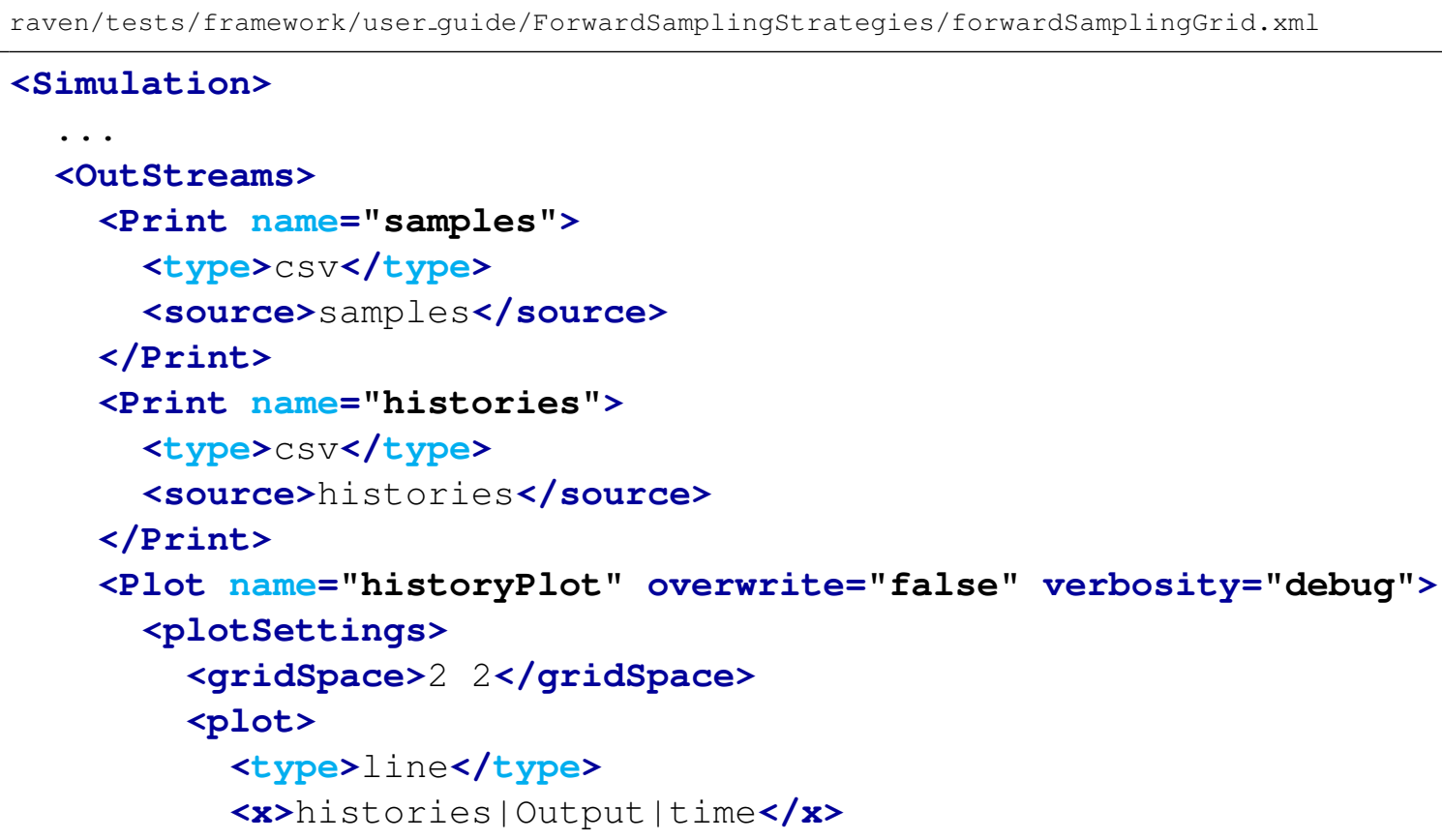




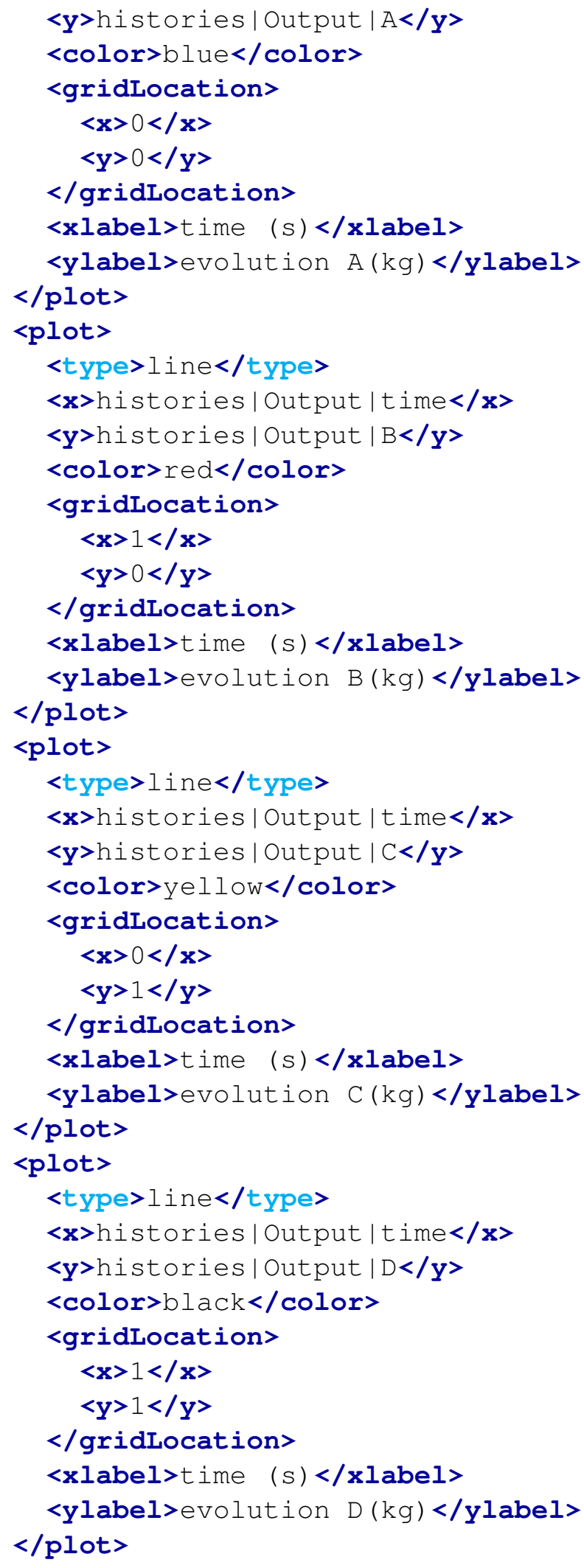




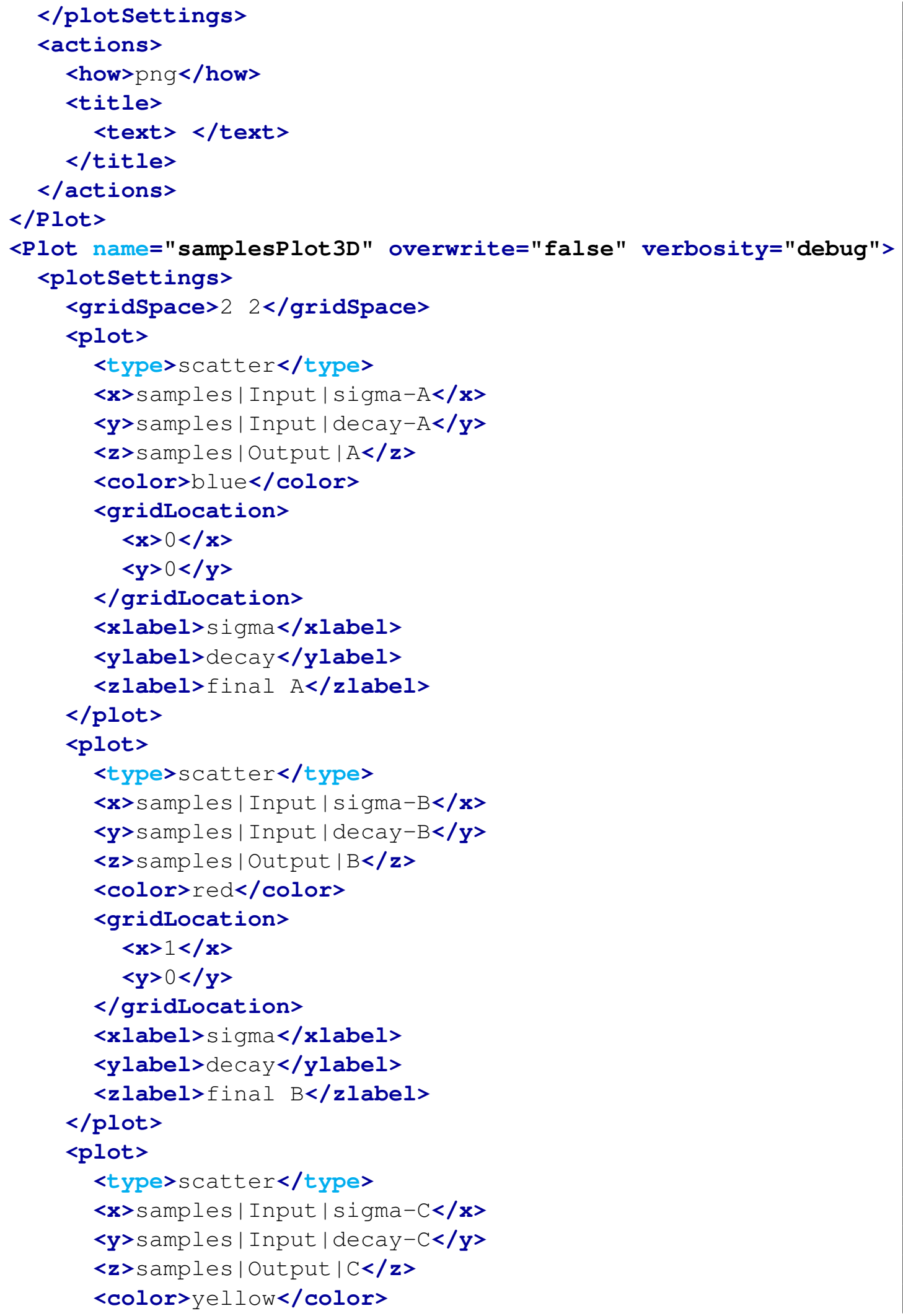




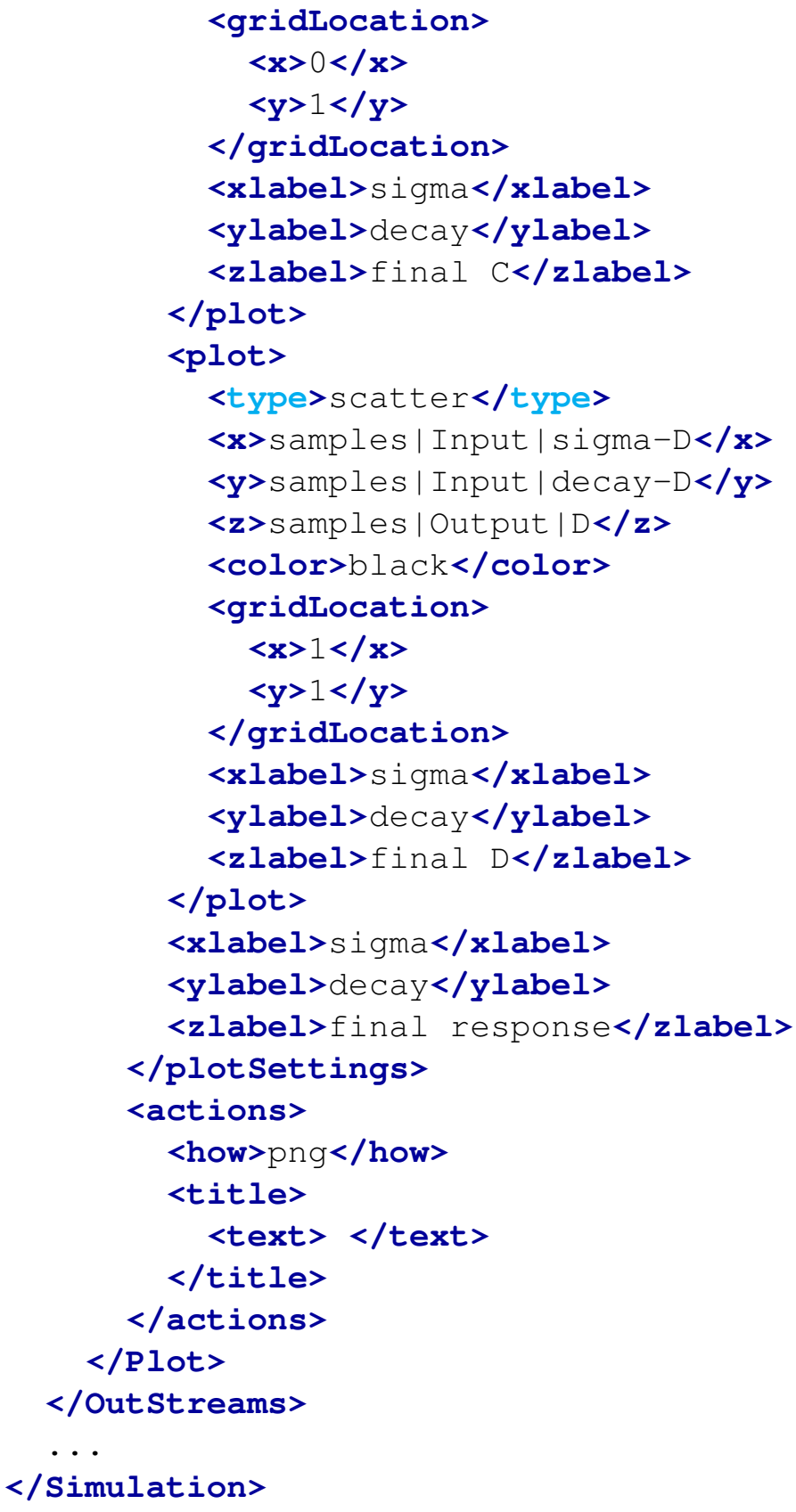

In this block, both the Out-Stream types are constructed:

- Print:

- named "samples" connected with the DataObjects Entity "samples" (<source>)

- named "histories" connected with the DataObjects Entity "histories" (<source>).

When these objects get used, all the information contained in the linked DataObjects are going to be exported in CSV files (<type>). 

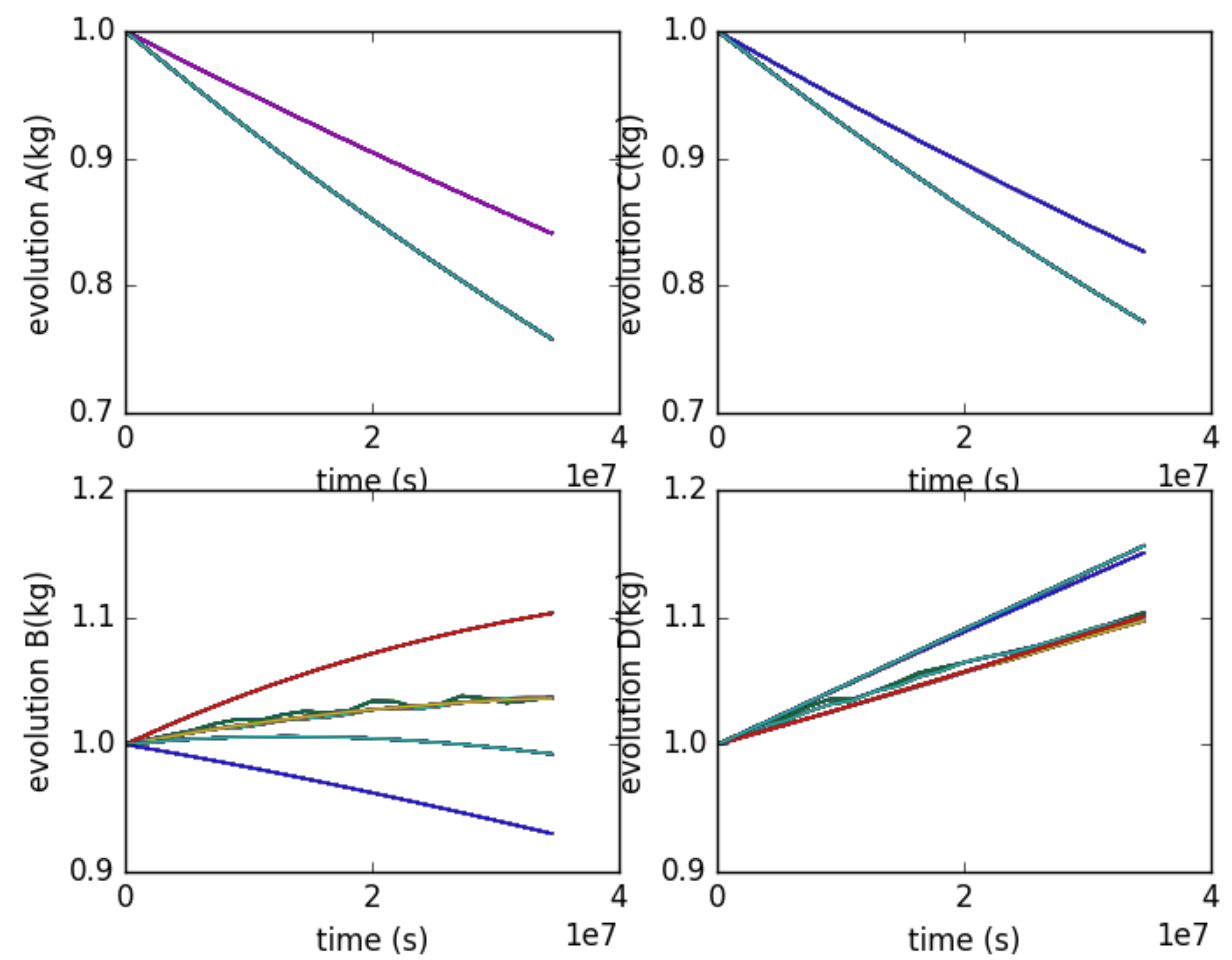

Figure 14. Plot of the histories generated by the Grid sampling for variables $A, B, C, D$.

- Plot:

- named "historiesPlot" connected with the DataObjects Entity "samples". This plot will draw the final state of the variables $A, B, C, D$ with respect to the input variables sigma(s) and decay(s).

- named "samplesPlot3D" connected with the DataObjects Entity "histories". This plot will draw the evolution of the variables $A, B, C, D$.

As it can be noticed, both plots are of type SubPlot. Four plots are placed in each of the figures.

\section{Steps:}

raven/tests/framework/user_guide/ForwardSamplingStrategies/forwardSamplingGrid.xml

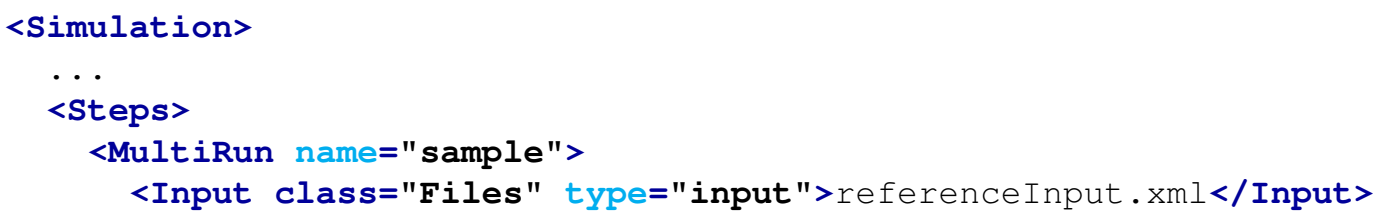



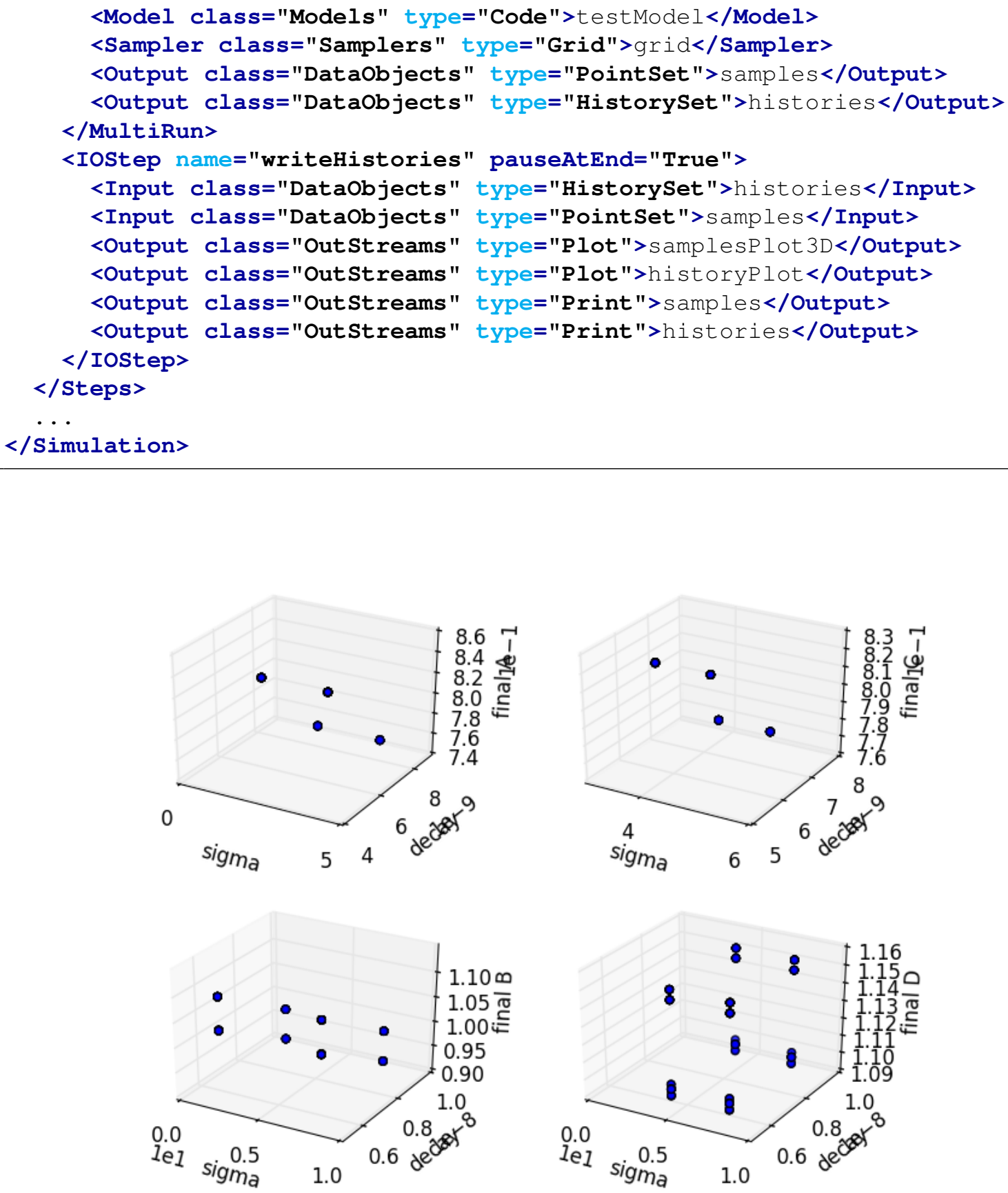

Figure 15. Plot of the samples generated by the Grid sampling for variables $A, B, C, D$.

Finally, all the previously defined Entities can be combined in the <Steps> block. As inferable, two <Steps > have been inputted: 
- <MultiRun> named "sample", is used to run the multiple instances of the code and collect the outputs in the two DataObjects. As it can be seen, the <Sampler> is inputted to communicate to the Step that the driven code needs to be perturbed through the Grid sampling

- <IOStep> named "writeHistories", used to 1) dump the "histories" and "samples" DataObjects Entity in a CSV file and 2) plot the data in the EPS file and on the screen.

Figures 14 and 15 report the evolution of the variables $A, B, C, D$ and their final values, respectively.

\subsection{Stratified sampling through RAVEN}

The Stratified sampling is a class of methods that relies on the assumption that the input space (i.e.,uncertainties) can be separated in regions (strata) based on similarity of the response of the system for input set within the same strata. Following this assumption, the most rewarding (in terms of computational cost vs. knowledge gain) sampling strategy would be to place one sample for each region. In this way, the same information is not collected more than once and all the prototypical behavior are sampled at least once. In Figure 16, the Stratified sampling approach is exemplified.
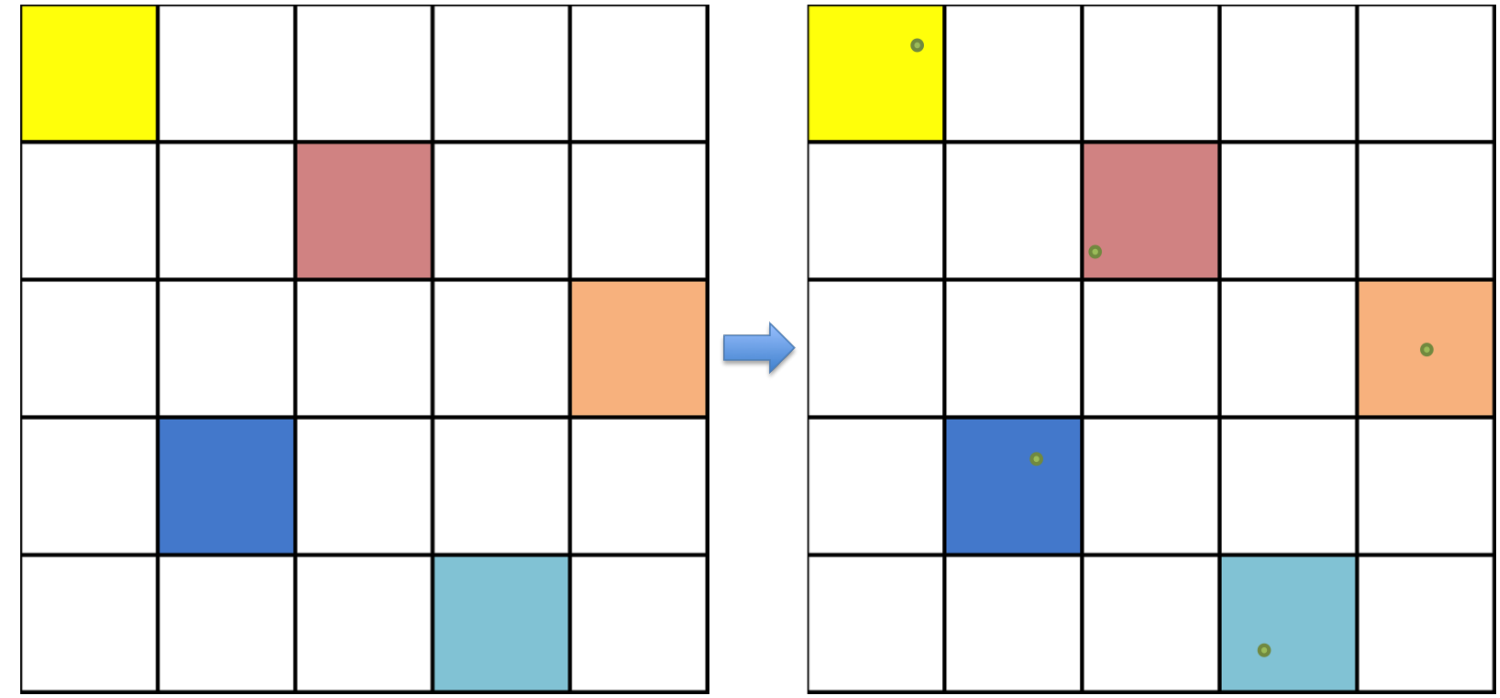

Figure 16. Example of Stratified sampling approach.

The goal of this section is to show how to: 
1. Set up a simple Stratified sampling in order to perform a parametric analysis on a driven code

2. Load the outputs of the code into the RAVEN DataObjects system

3. Print out what contained in the DataObjects

4. Generate basic plots of the code result.

To accomplish these tasks, the following RAVEN Entities (XML blocks in the input files) are defined:

\section{RunInfo:}

raven/tests/framework/user_guide/ForwardSamplingStrategies/forwardSamplingStratified.xml

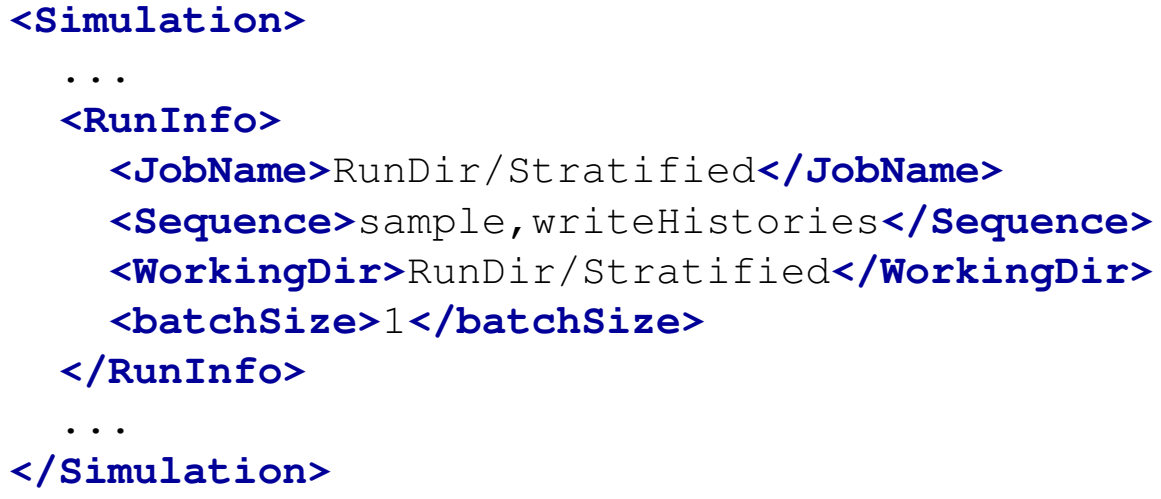

As reported in Section ??, the RunInfo Entity is intended to set up the analysis that the user wants to perform. In this specific case, two steps (<Sequence $>$ ) are run using 12 processors (<batchsize>). This means that 12 instances of the driven code are run simultaneously. Every time a simulation ends, a new one is launched.

\section{Files:}

raven/tests/framework/user_guide/ForwardSamplingStrategies/forwardSamplingStratified.xml

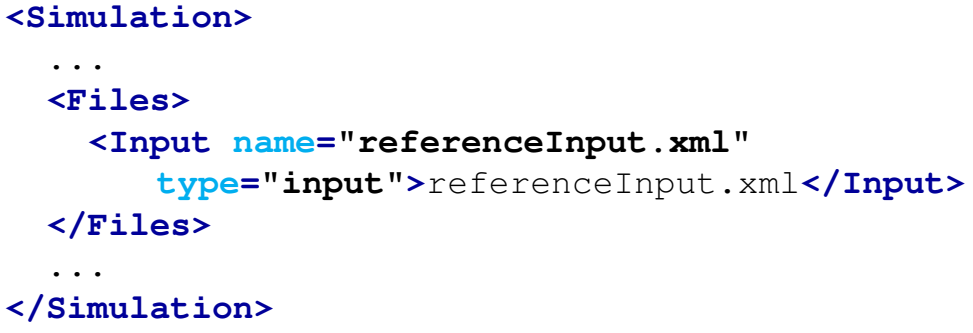

Since the considered code uses a single input file, in this section the original input is placed. The attribute name represents the alias that is going to be used in all the other input blocks in order to refer to this file. 


\section{Models:}

raven/tests/framework/user_guide/ForwardSamplingStrategies/forwardSamplingStratified.xml

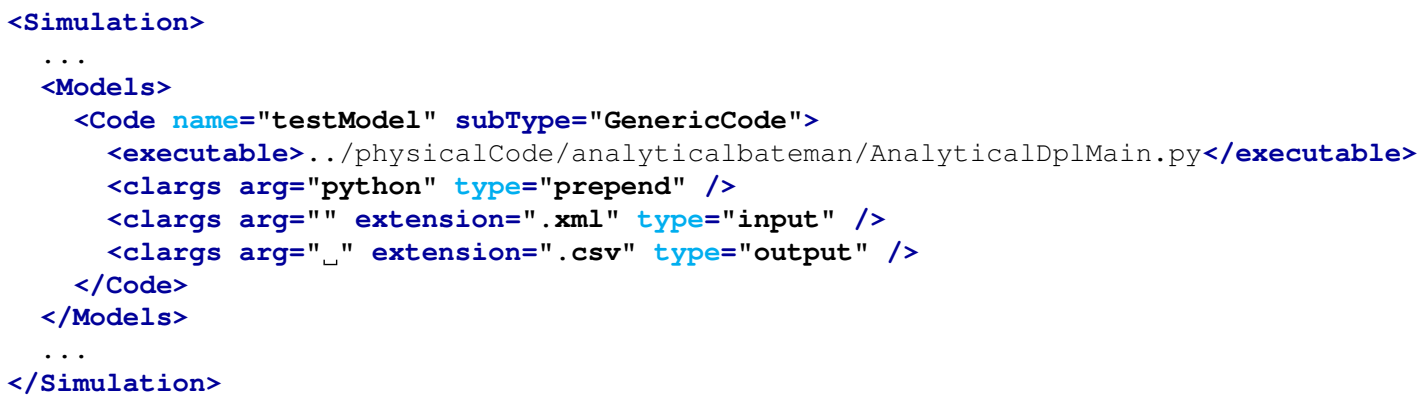

The Model here is represented by the AnalyticalBateman, which already dumps its output file in a CSV format (standard format that RAVEN can read). For this reason, the GenericCode interface is used.

\section{Distributions:}

raven/tests/framework/user_guide/ForwardSamplingStrategies/forwardSamplingStratified.xml

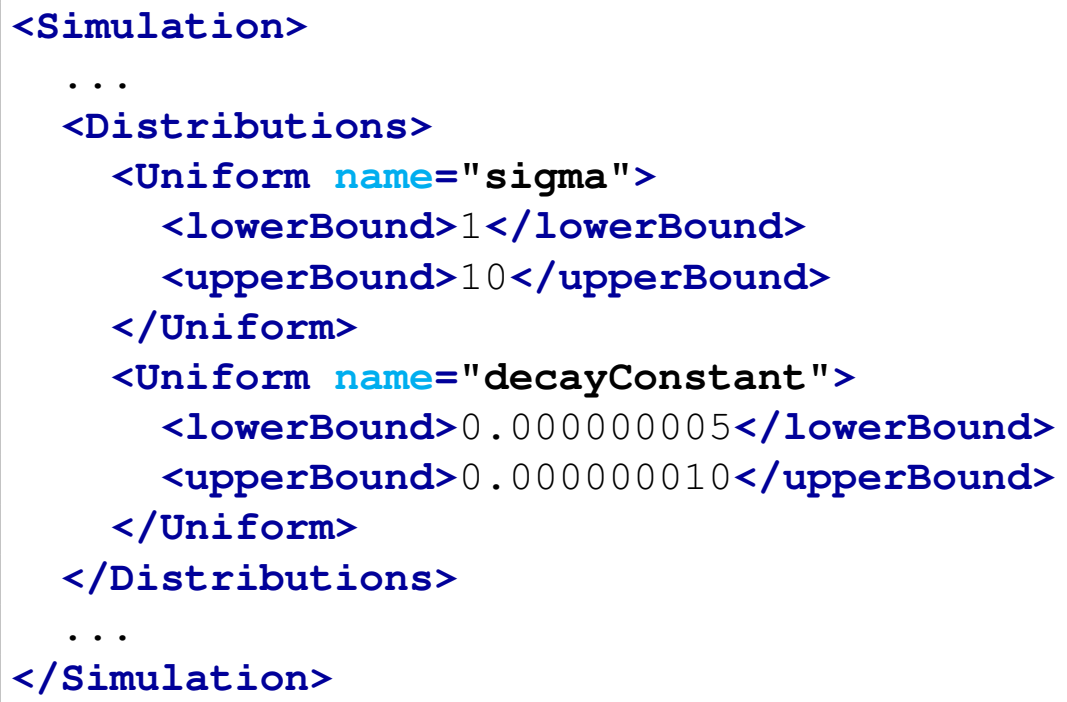

In the Distributions XML section, the stochastic model for the uncertainties treated by the Stratified sampling are reported. In this case two distributions are defined:

- sigma $\sim \mathbb{U}(1,10)$, used to model the uncertainties associated with the Model sigma(s)

- decayConstant $\sim \mathbb{U}(0.5 e-8,1 e-8)$, used to model the uncertainties associated with the Model decay constants.

\section{Samplers:}




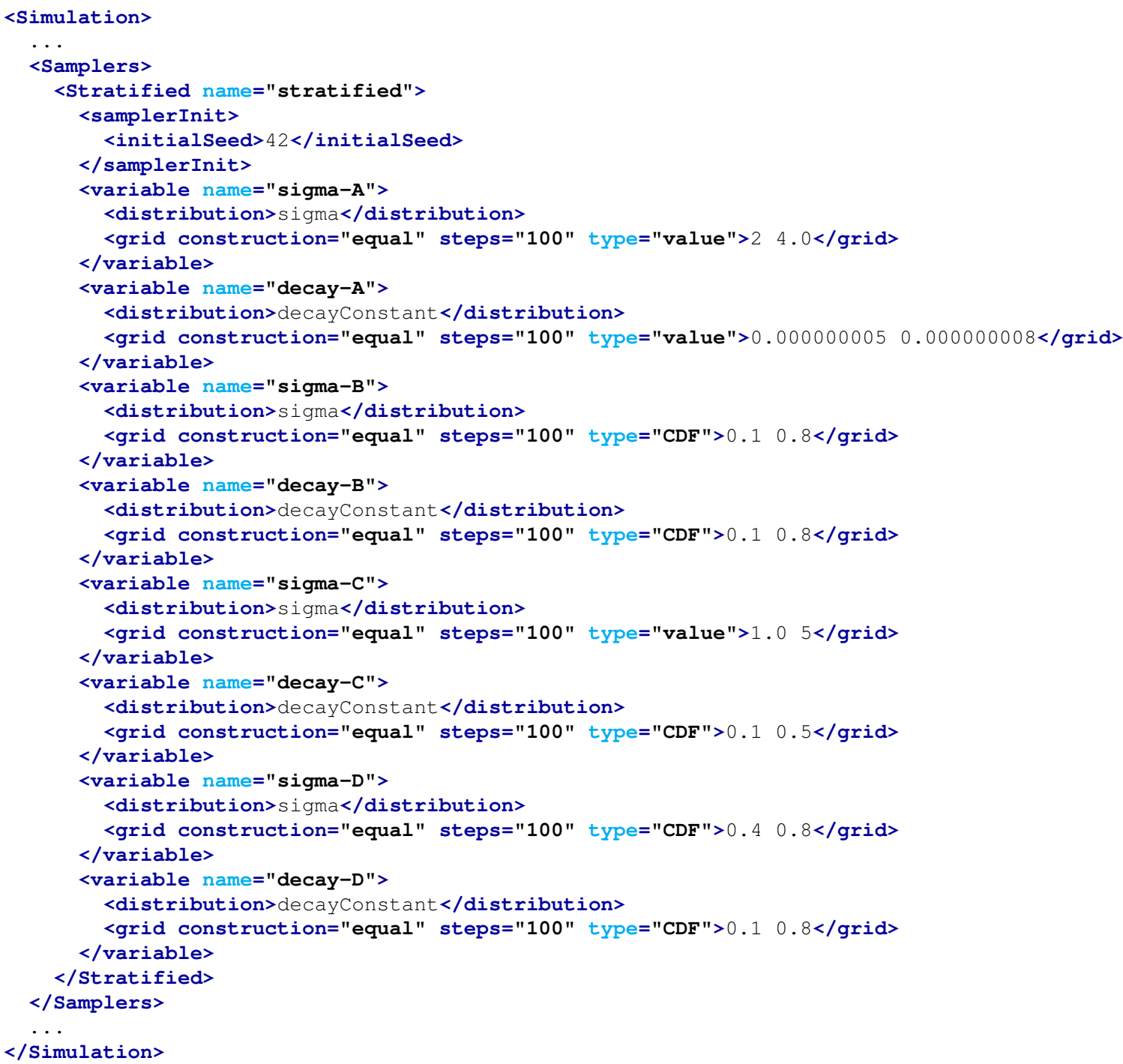

To employ the Stratified sampling strategy, a $<$ Stratified $>$ node needs to be specified. In each variable section, the $<g r i d>$ is defined. It is important to mention that the number of steps needs to be the same for each of the variables, since, as reported in previous section, the Stratified sampling strategy it discretizes the domain in strata. The number of samples finally requested is equal to $n_{\text {samples }}=n_{\text {steps }}=100$. If the grid for each variables is defined in CDF and of type = "equal", the Stratified sampling corresponds to the well-known Latin Hyper Cube sampling.

\section{DataObjects:}

raven/tests/framework/user_guide/ForwardSamplingStrategies/forwardSamplingStratified.xml

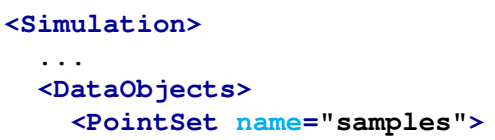




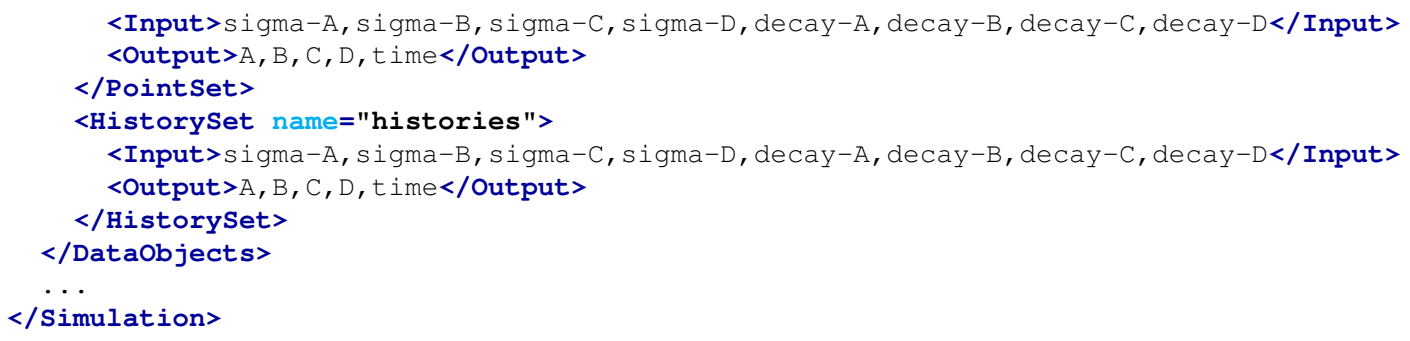

In this block, two DataObjects are defined: 1) PointSet named "samples", 2) HistorySet named "histories". In the <Input> node all the variables perturbed through the Stratified strategy are listed. In this way, any realization in the input space is linked to the outputs listed in the <Output> node.

\section{OutStreams:}

raven/tests/framework/user_guide/ForwardSamplingStrategies/forwardSamplingStratified.xml

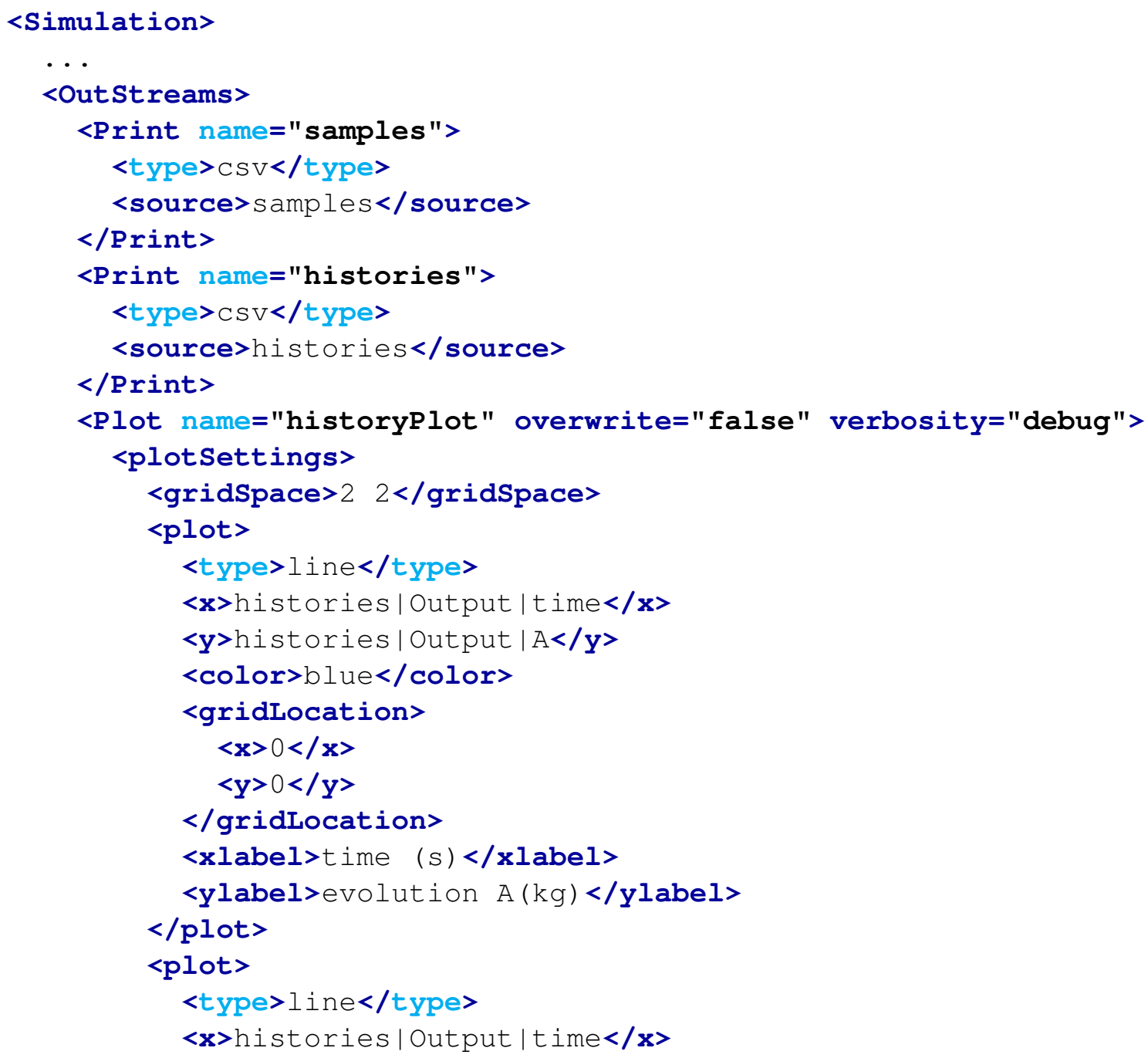




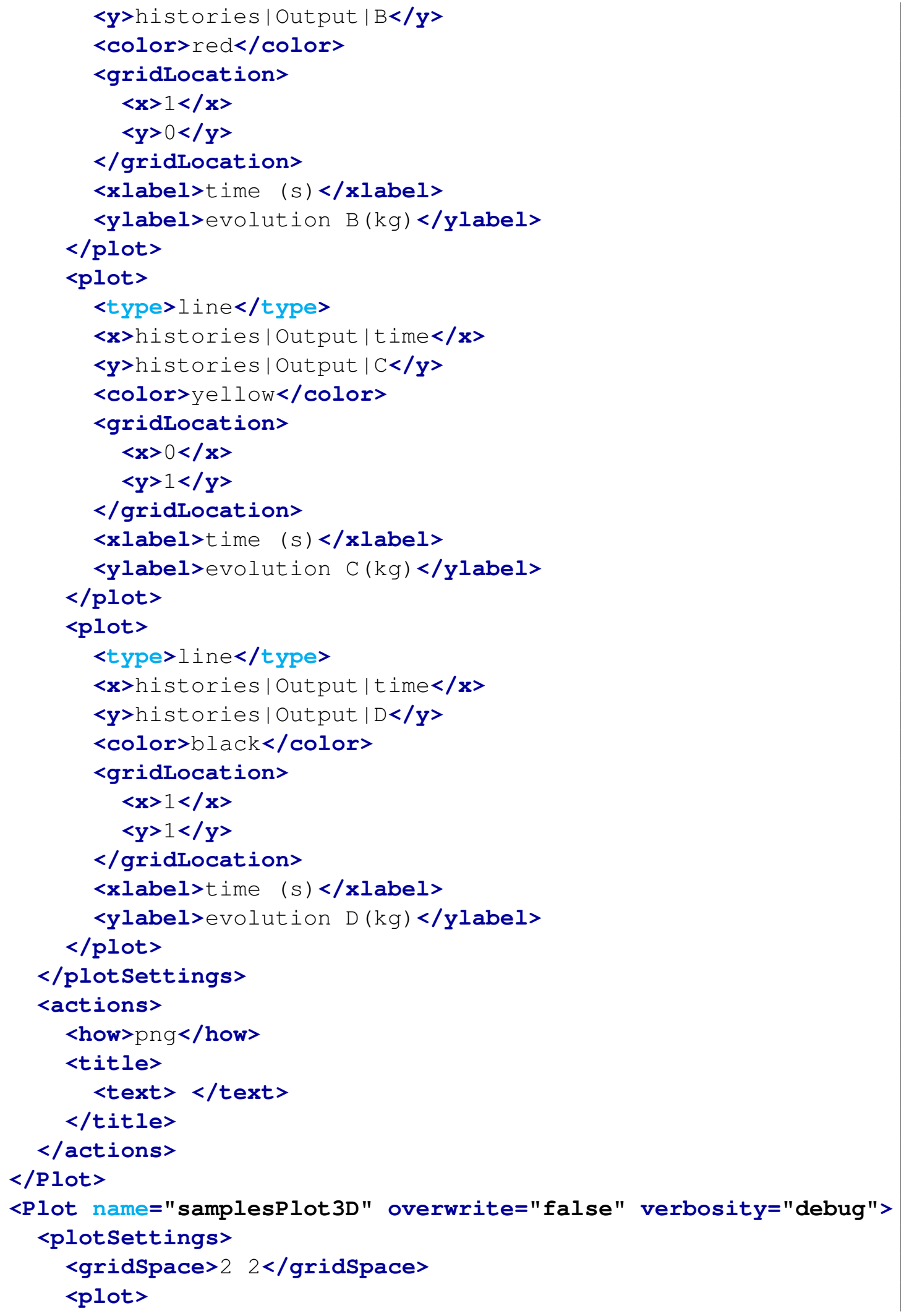




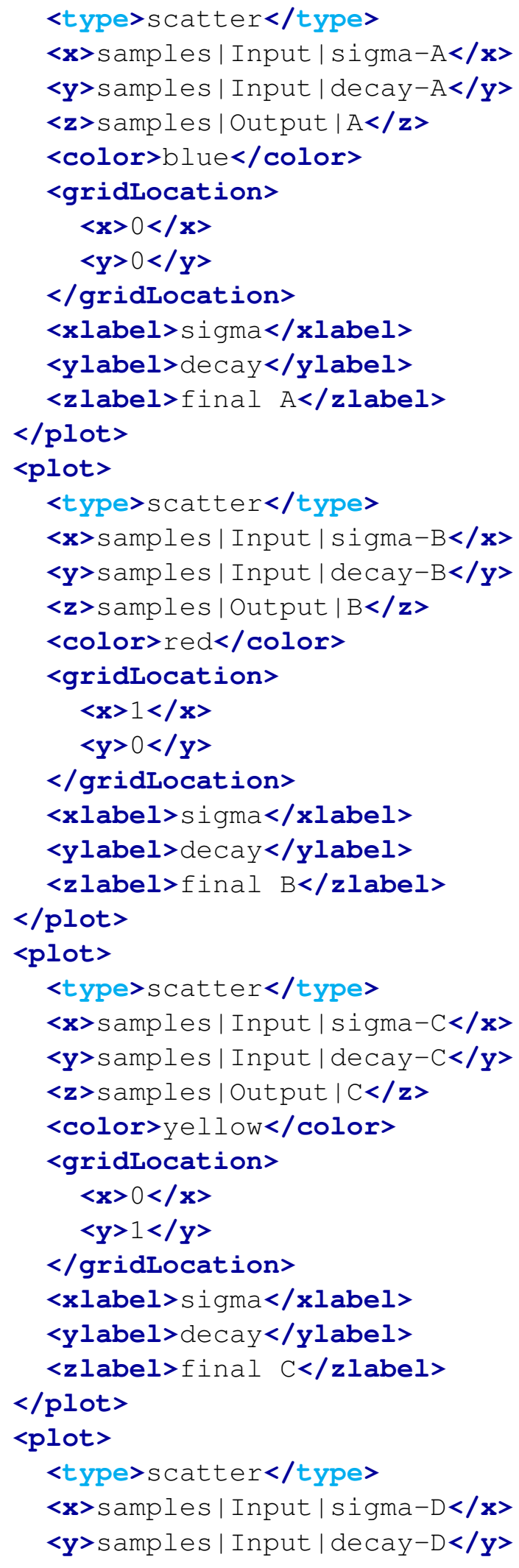




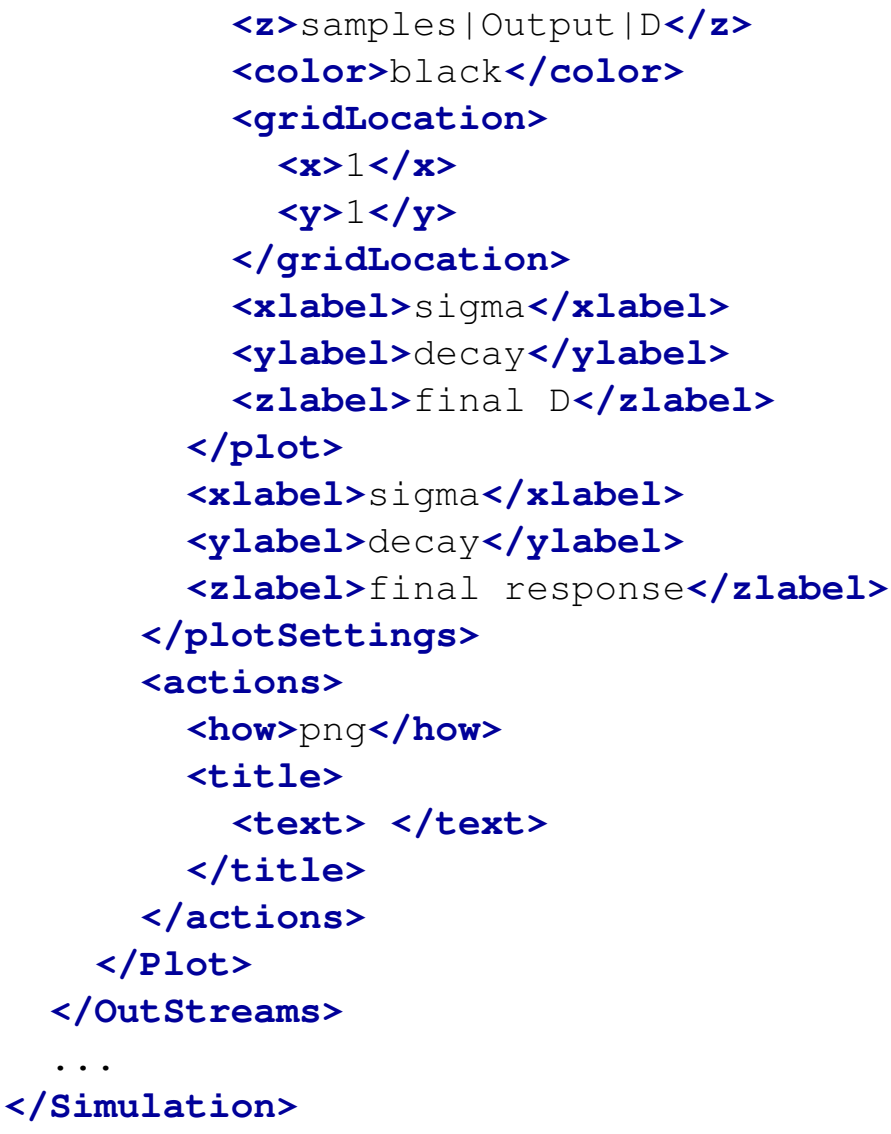

In this block, both the Out-Stream types are constructed:

- Print:

- named "samples" connected with the DataObjects Entity "samples" (<source>)

- named "histories" connected with the DataObjects Entity "histories" (<source>).

When these objects get used, all the information contained in the linked DataObjects are going to be exported in CSV files (<type>).

- Plot:

- named "historiesPlot" connected with the DataObjects Entity "samples". This plot will draw the final state of the variables $A, B, C, D$ with respect to the input variables sigma(s) and decay(s)

- named "samplesPlot3D" connected with the DataObjects Entity "histories". This plot will draw the evolution of the variables $A, B, C, D$.

As it can be noticed, both plots are of type SubPlot. Four plots are going to be placed in each of the figures.

\section{Steps:}



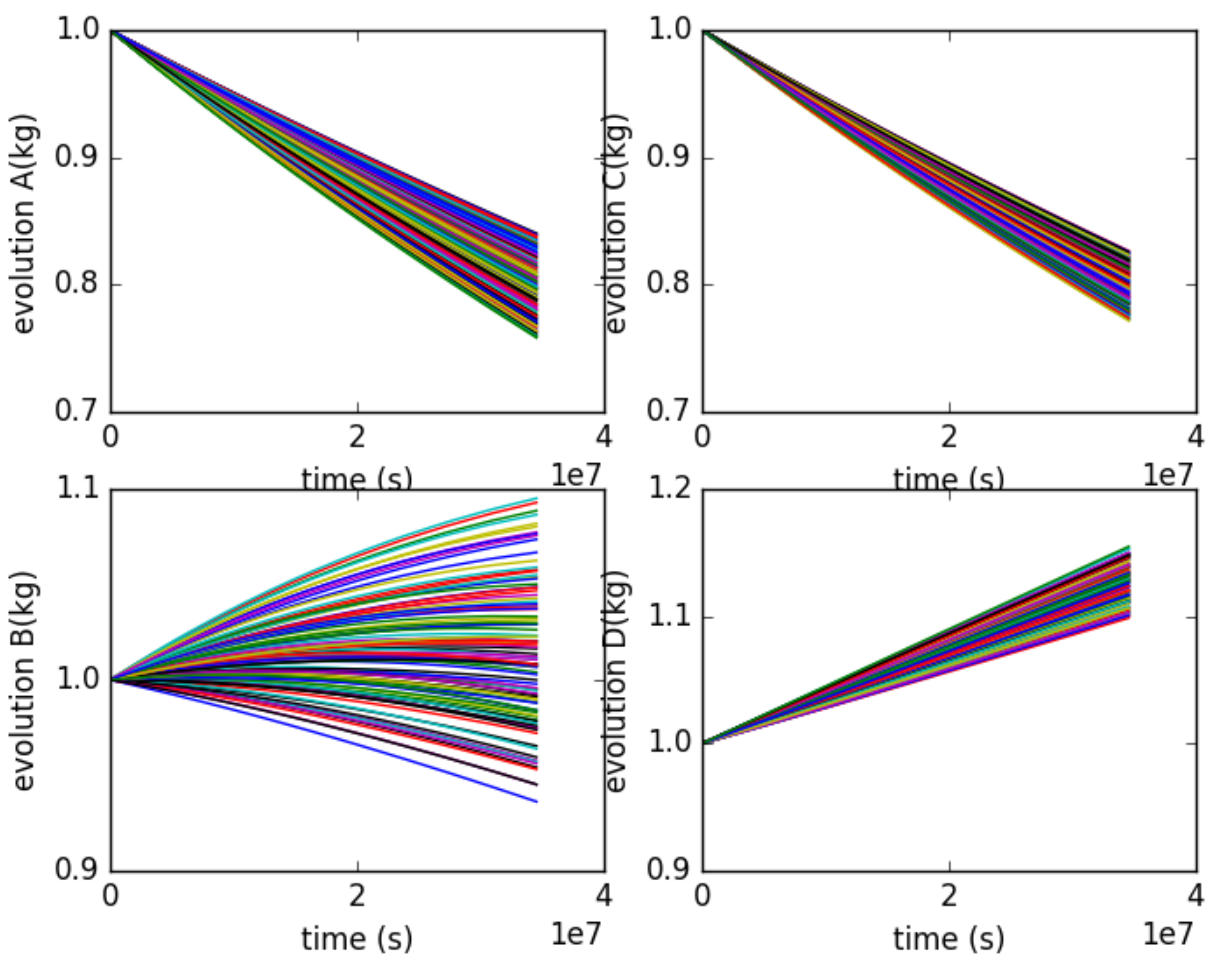

Figure 17. Plot of the histories generated by the Stratified sampling for variables $A, B, C, D$.

raven/tests/framework/user_guide/ForwardSamplingStrategies/forwardSamplingStratified.xml

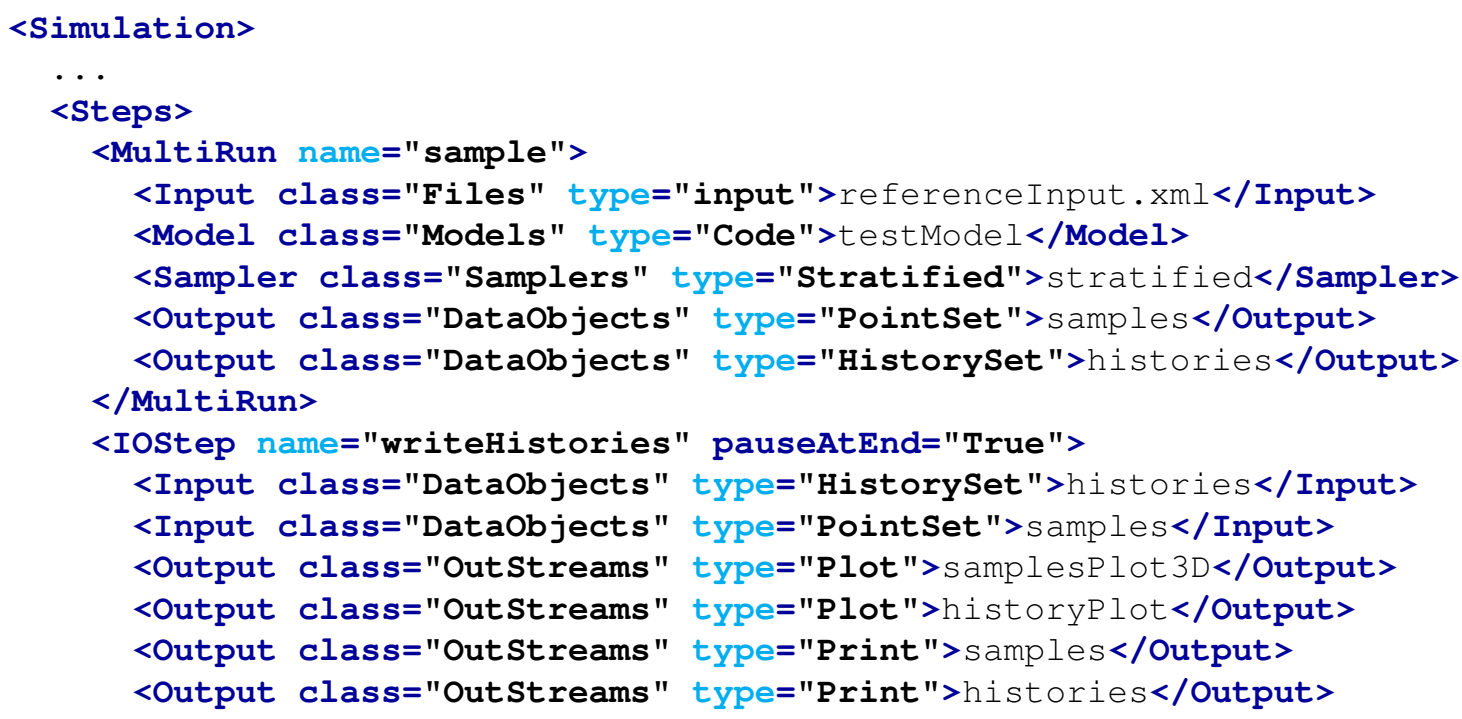


$</$ IOStep $>$

$</$ Steps $>$

...

$</$ Simulation $>$
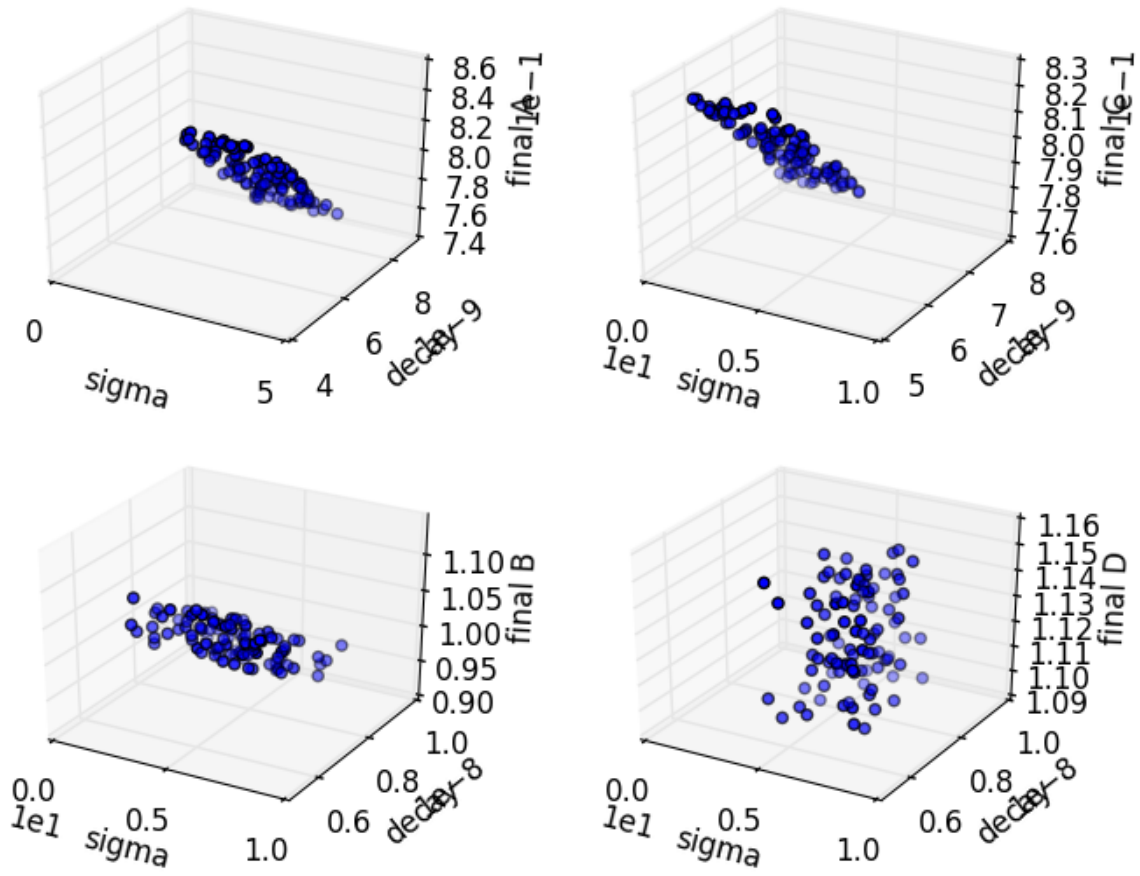

Figure 18. Plot of the samples generated by the Stratified sampling for variables $A, B, C, D$.

Finally, all the previously defined Entities can be combined in the <Steps $>$ block. As inferable, two <Steps $>$ have been inputted:

- <MultiRun> named "sample", used to run the multiple instances of the driven code and collect the outputs in the two DataObjects. As it can be seen, the <Sampler> is inputted to communicate to the Step that the driven code needs to be perturbed through the Stratified sampling.

- <IOStep> named "writeHistories", used to 1) dump the "histories" and "samples" DataObjects Entity in a CSV file and 2) plot the data in the EPS file and on the screen.

As previously mentioned, Figures 17 and 18 report the evolution of the variables $A, B, C, D$ and their final values, respectively. 


\subsection{Sparse Grid Collocation sampling through RAVEN}

The Sparse Grid Collocation sampler represents an advanced methodology to perform Uncertainty Quantification. They aim to explore the input space leveraging the information contained in the associated probability density functions. It builds on generic Grid sampling by selecting evaluation points based on characteristic quadratures as part of stochastic collocation for generalized polynomial chaos uncertainty quantification. In collocation an N-D grid is constructed, with each uncertain variable providing an axis. Along each axis, the points of evaluation correspond to quadrature points necessary to integrate polynomials. In the simplest (and most naive) case, a N-D tensor product of all possible combinations of points from each dimension's quadrature is constructed as sampling points. The number of necessary samples can be reduced by employing Smolyak-like sparse grid algorithms, which use reduced combinations of polynomial orders to reduce the necessary sampling space.

The goals of this section are about learning how to:

1. Set up a Sparse Grid Collocation sampling for the construction of a suitable surrogate model of a driven code

2. Construct a GaussPolynomialRom surrogate model (training stage)

3. Use the constructed GaussPolynomialRom surrogate model instead of the driven code.

To accomplish these tasks, the following RAVEN Entities (XML blocks in the input files) need to be defined:

\section{RunInfo:}

raven/tests/framework/user_guide/ForwardSamplingStrategies/forwardSamplingSparseGrid.xml

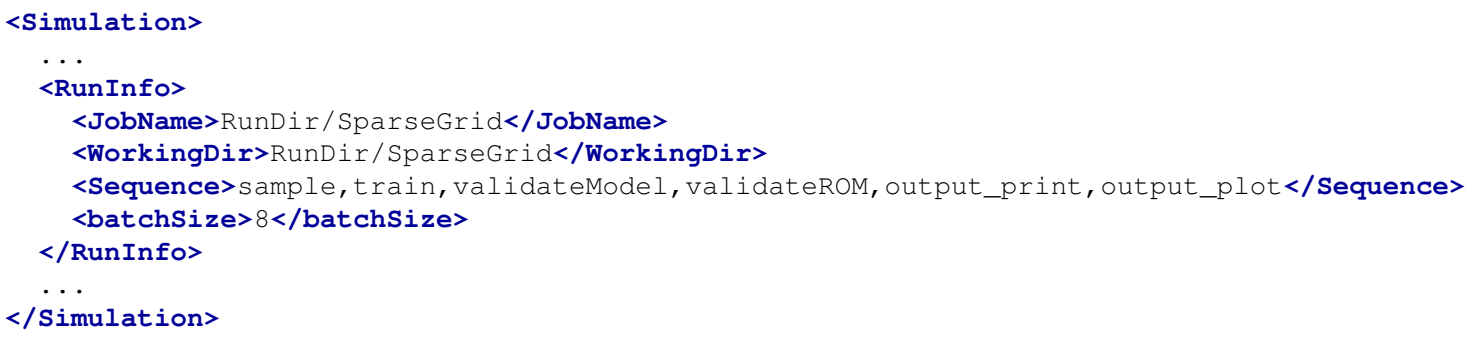

As reported in section ??, the RunInfo Entity is intended to set up the analysis that the user wants to perform. In this specific case, six steps (<Sequence>) are going to be sequentially run using twelve processors (<batchSize>). The first two steps build the ROM (' sample', 'train'), the next two validate the ROM against the original Code Model ('validateModel', 'validateROM'), and the last two produce plots and print data (' output_print', ' output_plot'). 


\section{Files:}

raven/tests/framework/user_guide/ForwardSamplingStrategies/forwardSamplingSparseGrid.xml

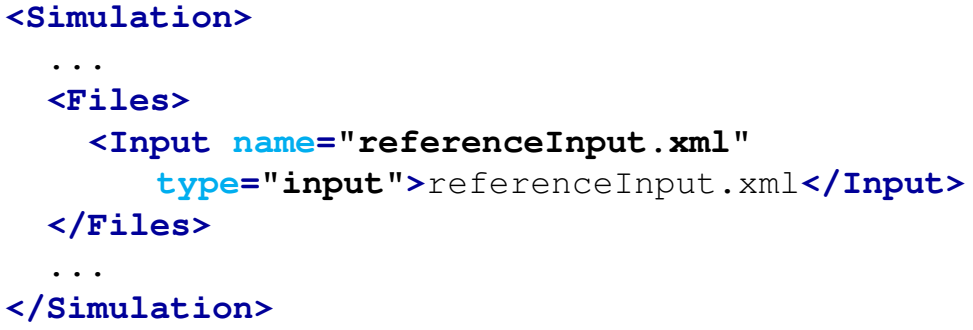

Since the driven code uses a single input file, in this section the original input is placed. As detailed in the user manual the attribute name represents the alias that is going to be used in all the other input blocks in order to refer to this file.

\section{Models:}

raven/tests/framework/user_guide/ForwardSamplingStrategies/forwardSamplingSparseGrid.xml

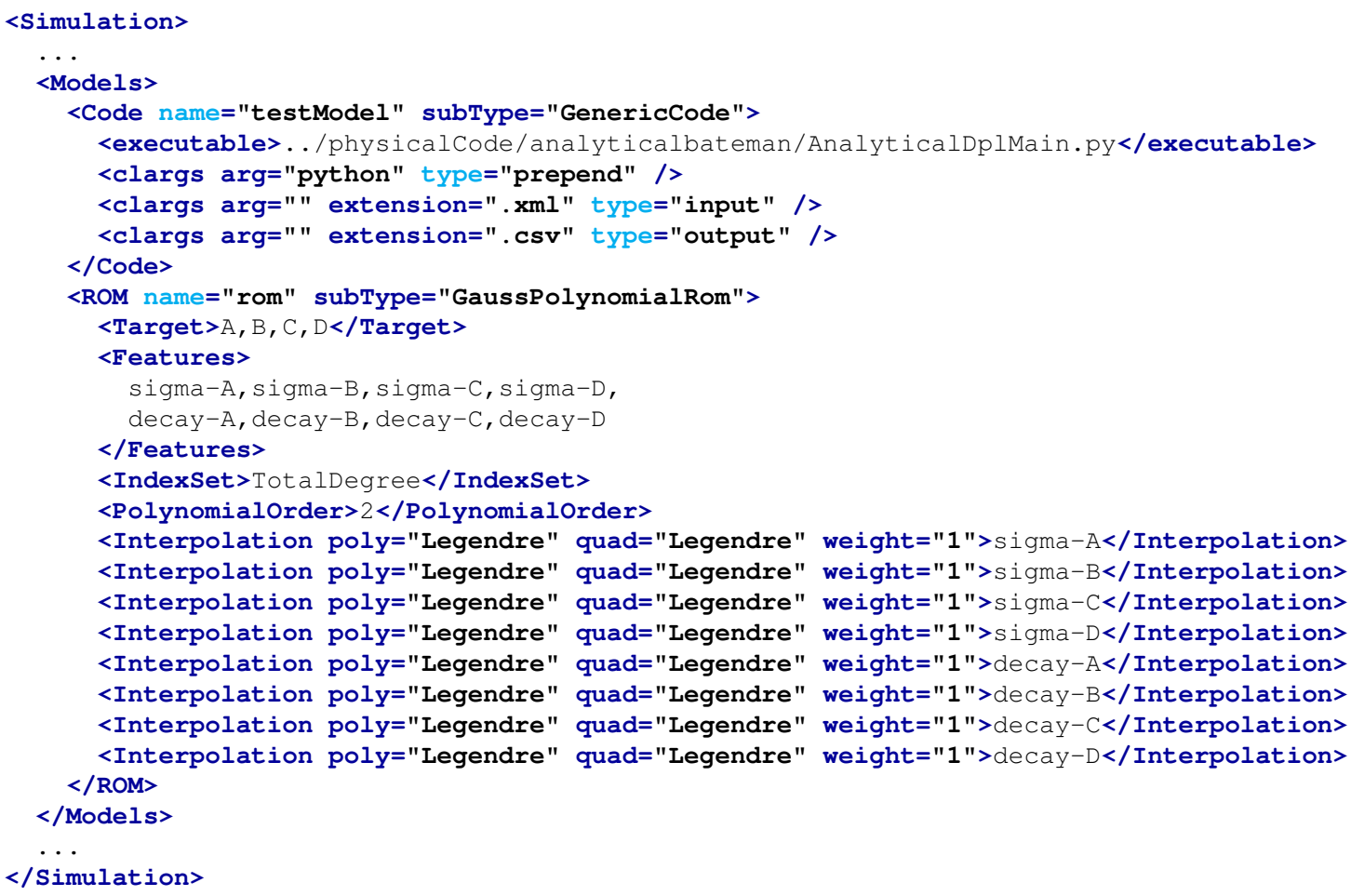

The goal of this example is the generation of a GaussPolynomialRom for subsequent usage instead of the original code. In addition to the previously explained Code model, the ROM of type GaussPolynomialRom is specified here. The ROM is generated through a Sparse Grid Collocation sampling strategy. All 4 targets $A, B, C, D$ are modeled through this ROM as function of the uncertain sigma and decay parameters. Note that the <Interpolation> nodes are not required, but are included for the sake of demonstration. 


\section{Distributions:}

raven/tests/framework/user_guide/ForwardSamplingStrategies/forwardSamplingSparseGrid.xml

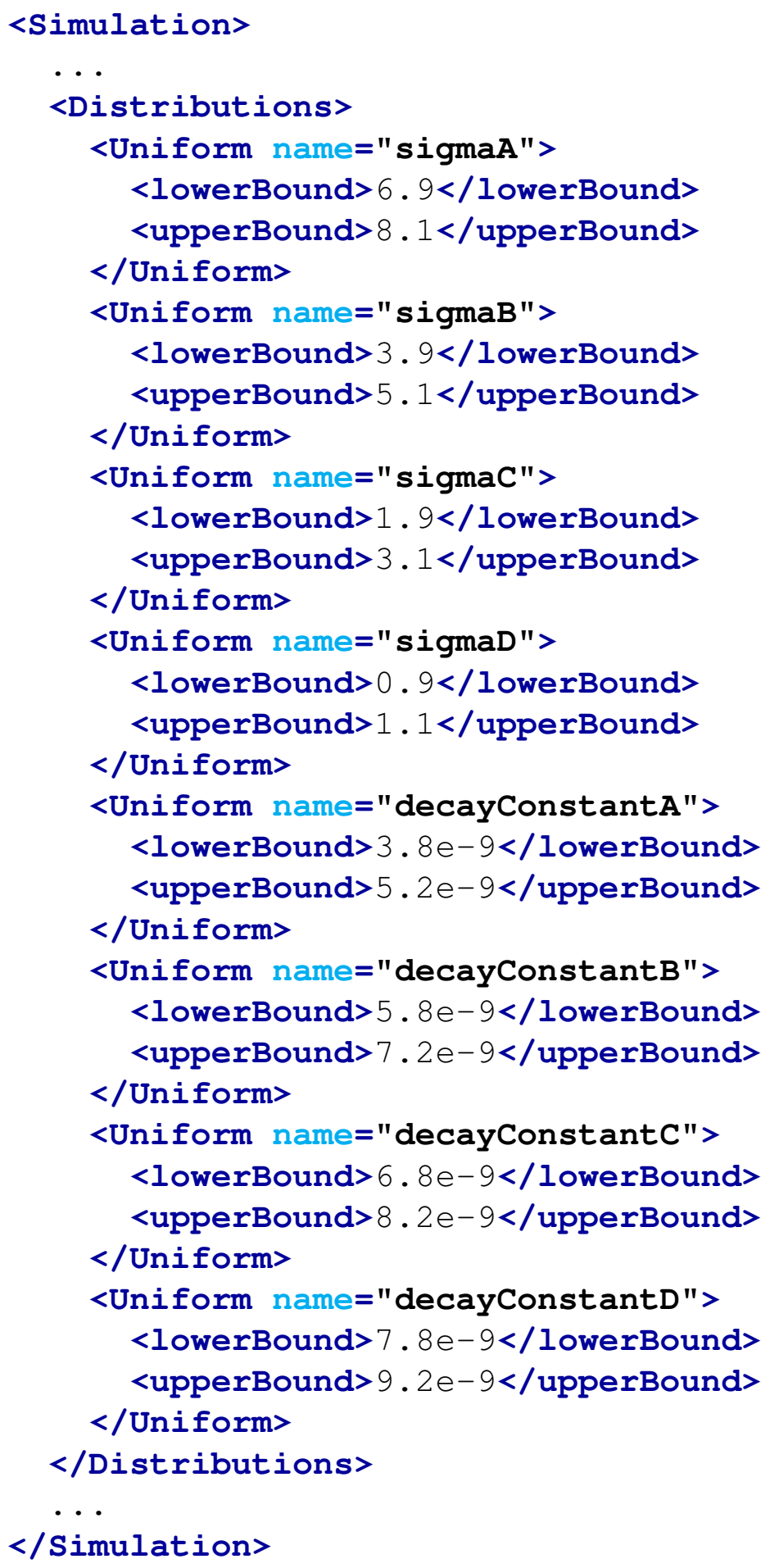

In the Distributions XML section, the stochastic model for the uncertainties treated by the 
Sparse Grid Collocation sampling are reported. In this case eight distributions are defined:

- sigmaA $\sim \mathbb{U}(6.9,8.1)$, used to model the uncertainty associated with the Model sigma- $A$

- sigmaB $\sim \mathbb{U}(3.9,5.1)$, used to model the uncertainty associated with the Model sigma-B

- sigmaC $\sim \mathbb{U}(1.9,3.1)$, used to model the uncertainty associated with the Model sigma-C

- sigmaD $\sim \mathbb{U}(0.9,1.1)$, used to model the uncertainty associated with the Model sigma-D

- decayConstant $A \sim \mathbb{U}(3.8 e-9,5.2 e-9)$, used to model the uncertainty associated with the Model decay- $A$

- decayConstant $B \sim \mathbb{U}(5.8 e-9,7.2 e-9)$, used to model the uncertainty associated with the Model decay-B

- decayConstant $C \sim \mathbb{U}(6.8 e-9,8.2 e-9)$, used to model the uncertainty associated with the Model decay-C

- decayConstant $D \sim \mathbb{U}(7.8 e-9,9.2 e-9)$, used to model the uncertainty associated with the Model decay-D.

\section{Samplers:}

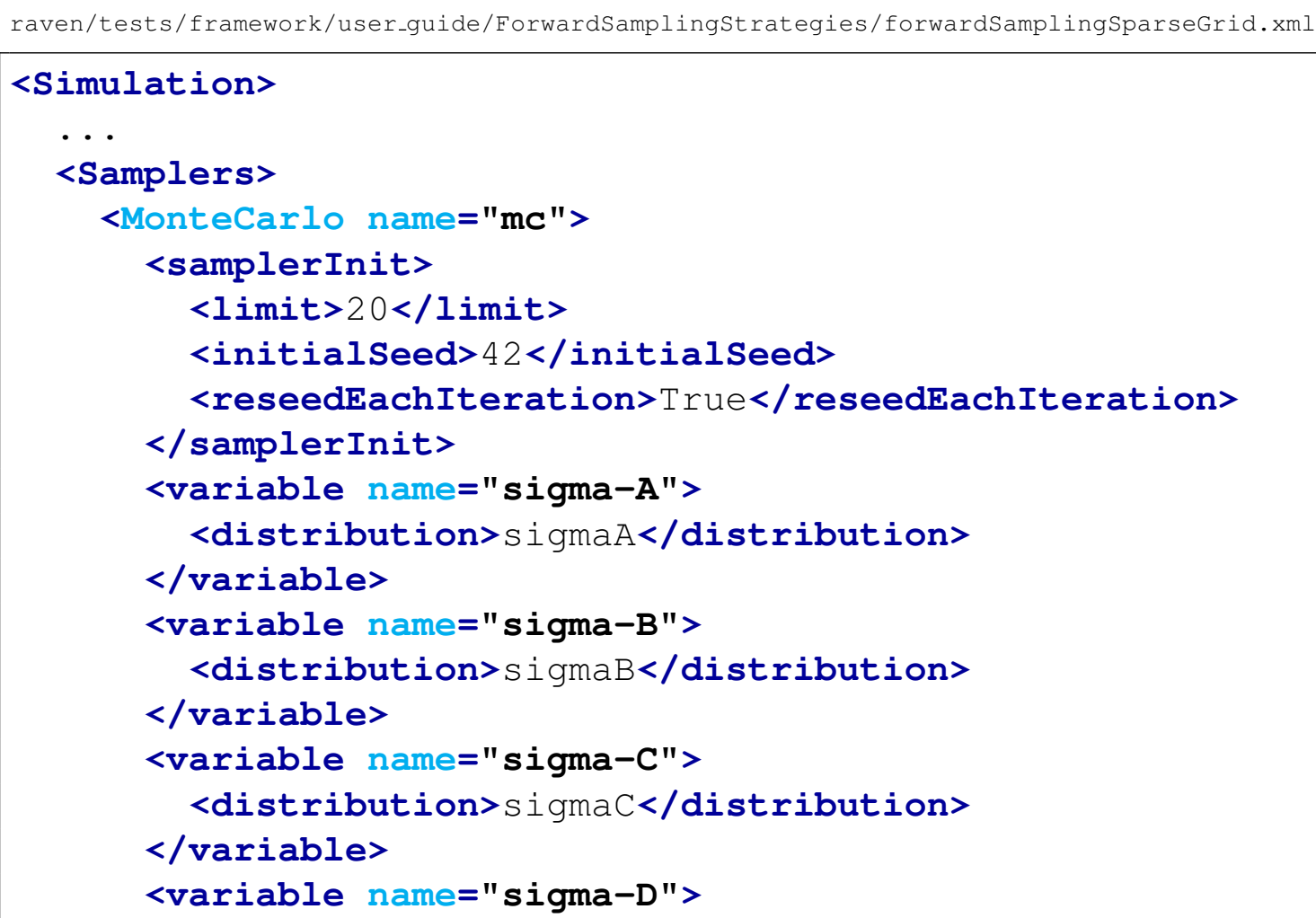


<distribution>sigmaD </distribution>

$<$ /variable>

<variable name="decay-A">

<distribution>decayConstant $A</$ distribution>

</variable>

<variable name="decay-B">

<distribution>decayConstant $\mathrm{B}</$ distribution $>$

</variable>

<variable name="decay-C">

<distribution>decayConstantC</distribution>

</variable>

<variable name="decay-D">

<distribution>decayConstant $\mathrm{D}</$ distribution $>$ $</$ variable>

</MonteCarlo>

<SparseGridCollocation name="SG">

<variable name="sigma-A">

<distribution>sigmaA </distribution>

$</$ variable>

<variable name="sigma-B">

<distribution>sigmaB $</$ distribution>

</variable>

<variable name="sigma-C">

<distribution>sigmaC $</$ distribution>

</variable>

<variable name="sigma-D">

<distribution>sigmaD </distribution>

</variable>

<variable name="decay-A">

<distribution>decayConstant $A</$ distribution $>$

</variable>

<variable name="decay-B">

<distribution>decayConstant $\mathrm{B}</$ distribution $>$

</variable>

<variable name="decay-C">

<distribution>decayConstantC</distribution>

</variable>

<variable name="decay-D">

<distribution>decayConstantD</distribution>

$</ v a r i a b l e>$

<ROM class="Models" type="ROM" >rom</ROM>

</SparseGridCollocation> 
...

$</$ Simulation $>$

In order to employ the Sparse Grid Collocation sampling strategy, a <SparseGridCollocation> node needs to be defined. As can be seen from above, each variable is associated with a different distribution, defined in the $<$ Distributions $>$ block. In addition, the GaussPolynomialRom <ROM> is linked to the <SparseGridCollocation> sampler. Because this sampler is used exclusively to build the ROM, some of the parameters of the ROM are needed by the sampler, and this connection makes that communication possible. The setting of this ROM (e.g. polynomial order, Index set method, etc.) determines how the Stochastic Collocation Method is employed.

Additionally, a <MonteCarlo> sampler is set up for validating the ROM against the original Code. The random number generation seed (<initialseed>) is specified and set to reset on each use (<reseedEachIteration $>$ ) so that the Monte Carlo sampler can be used to compare the ROM against the original model. We use twenty samples (<limit $>$ ) to sample the ROM and the model, and then print and plot both data sets to compare them.

\section{DataObjects:}

raven/tests/framework/user_guide/ForwardSamplingStrategies/forwardSamplingSparseGrid.xml

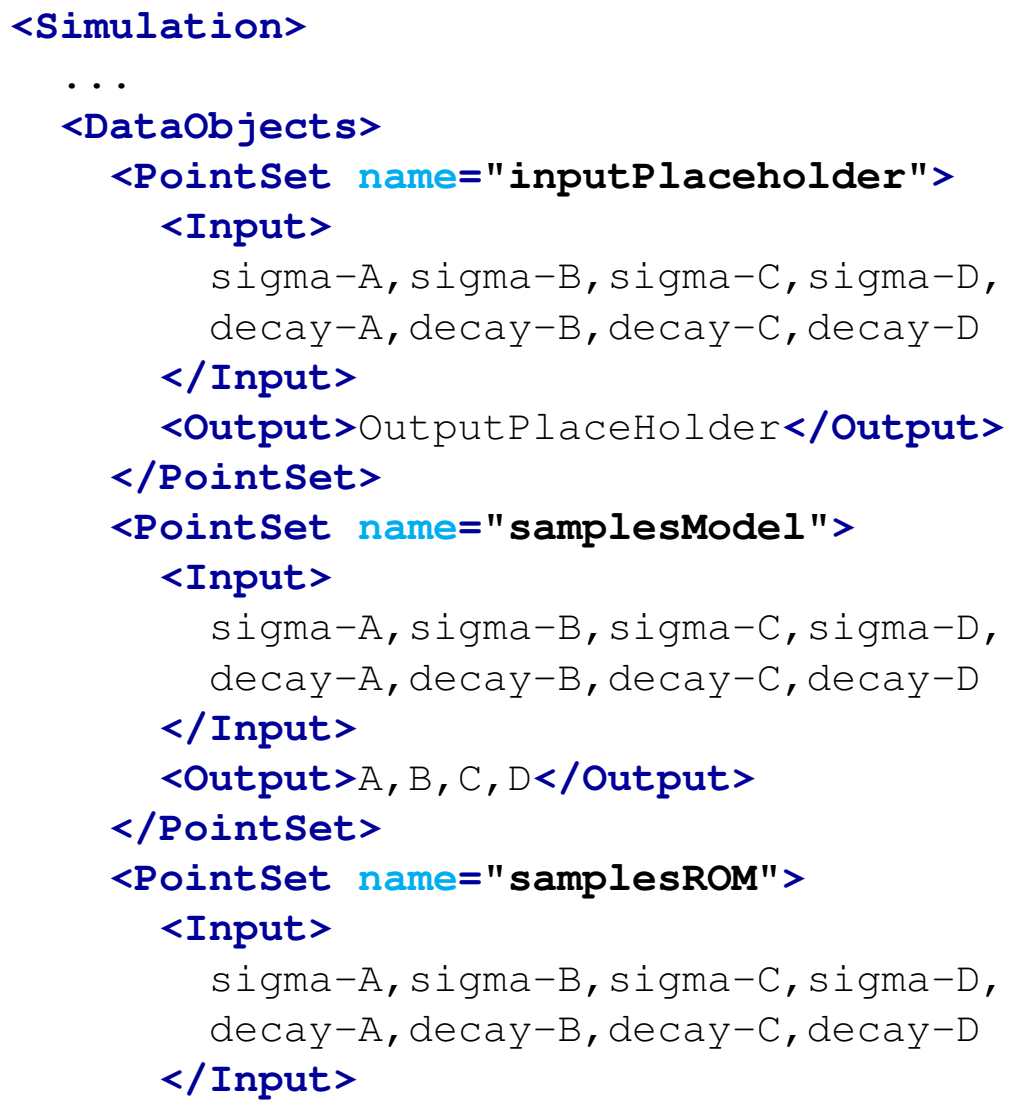




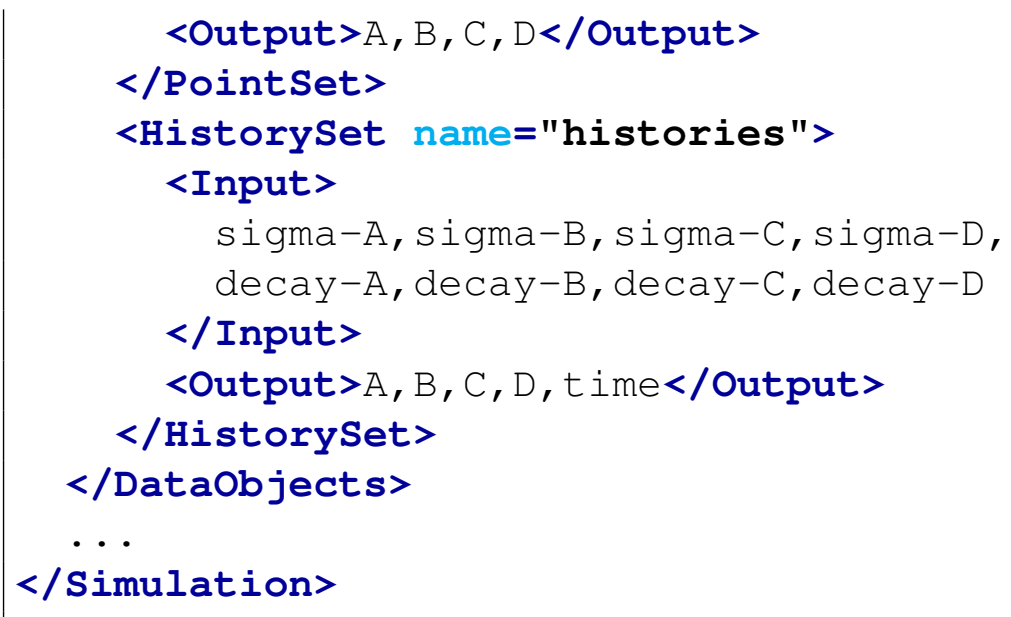

In this block, four DataObjects are defined: 1) a PointSet named "inputPlaceholder" used as a placeholder input for the ROM sampling step, 2) a HistorySet named "histories" used to collect the samples needed to train the ROM, 3) a PointSet named "samplesModel" to store the Code responses from Monte Carlo samples, and 4) a PointSet named "samplesROM" to store the ROM responses from Monte Carlo samples.

\section{OutStreams:}



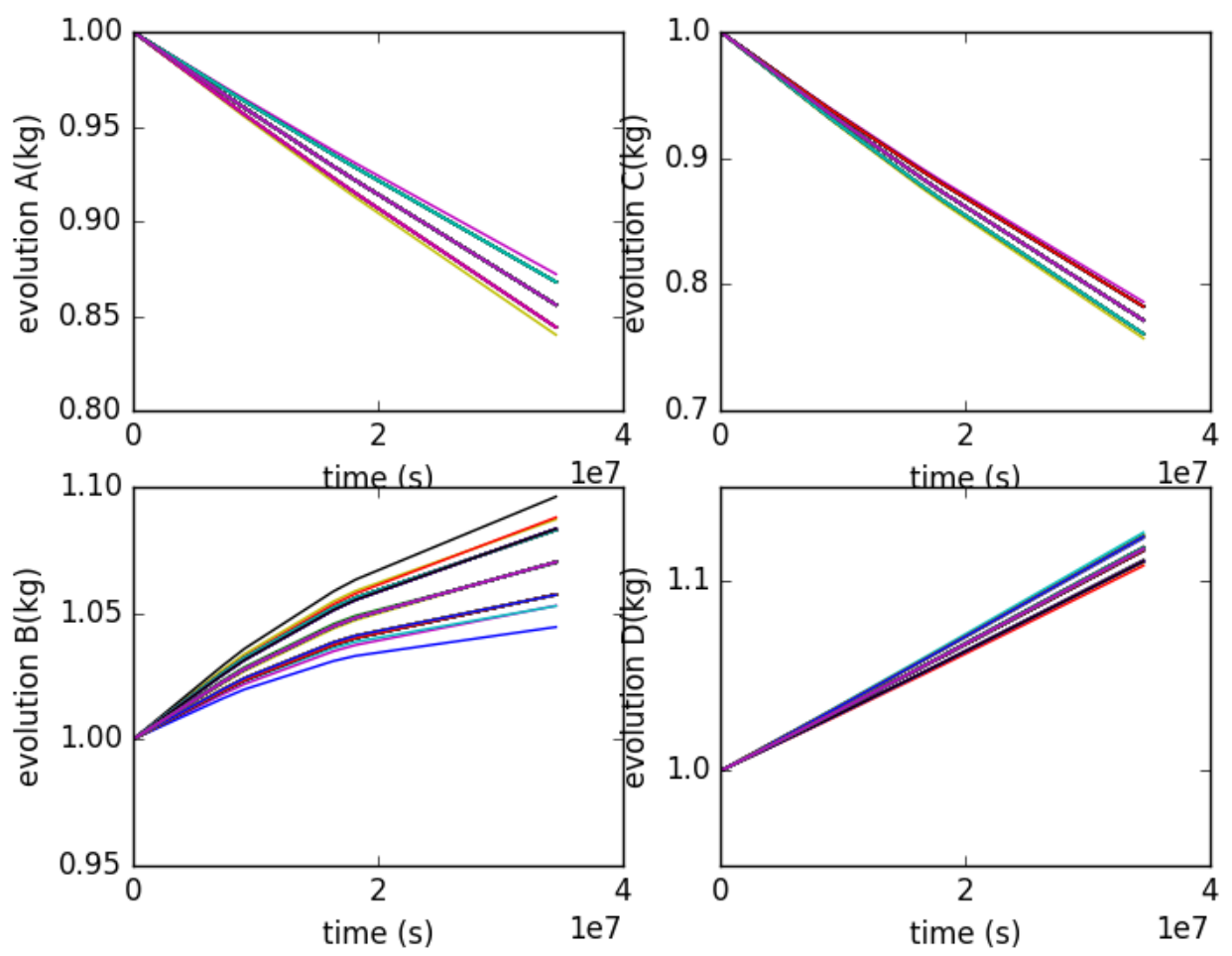

Figure 19. Plot of the training samples generated by the SparseGridCollocation sampling for variables $A, B, C, D$.

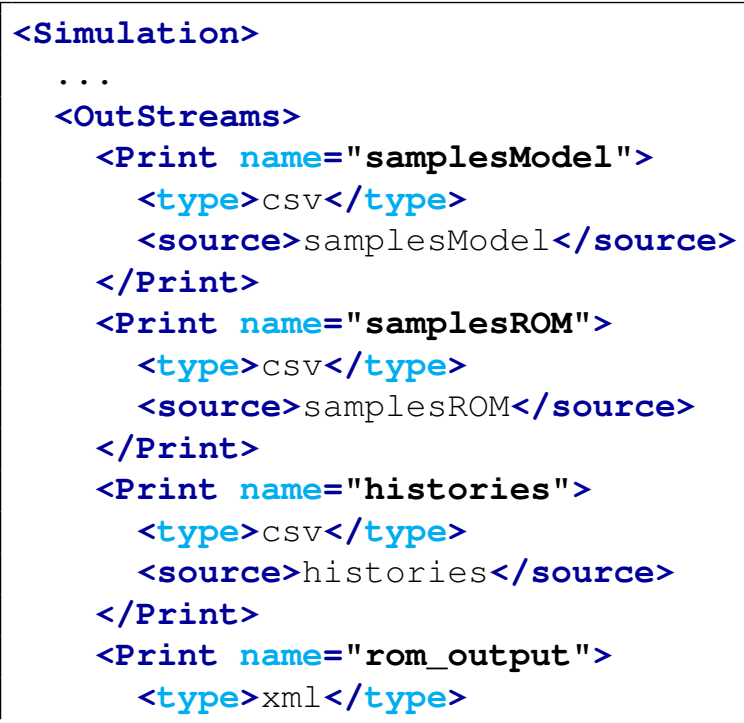




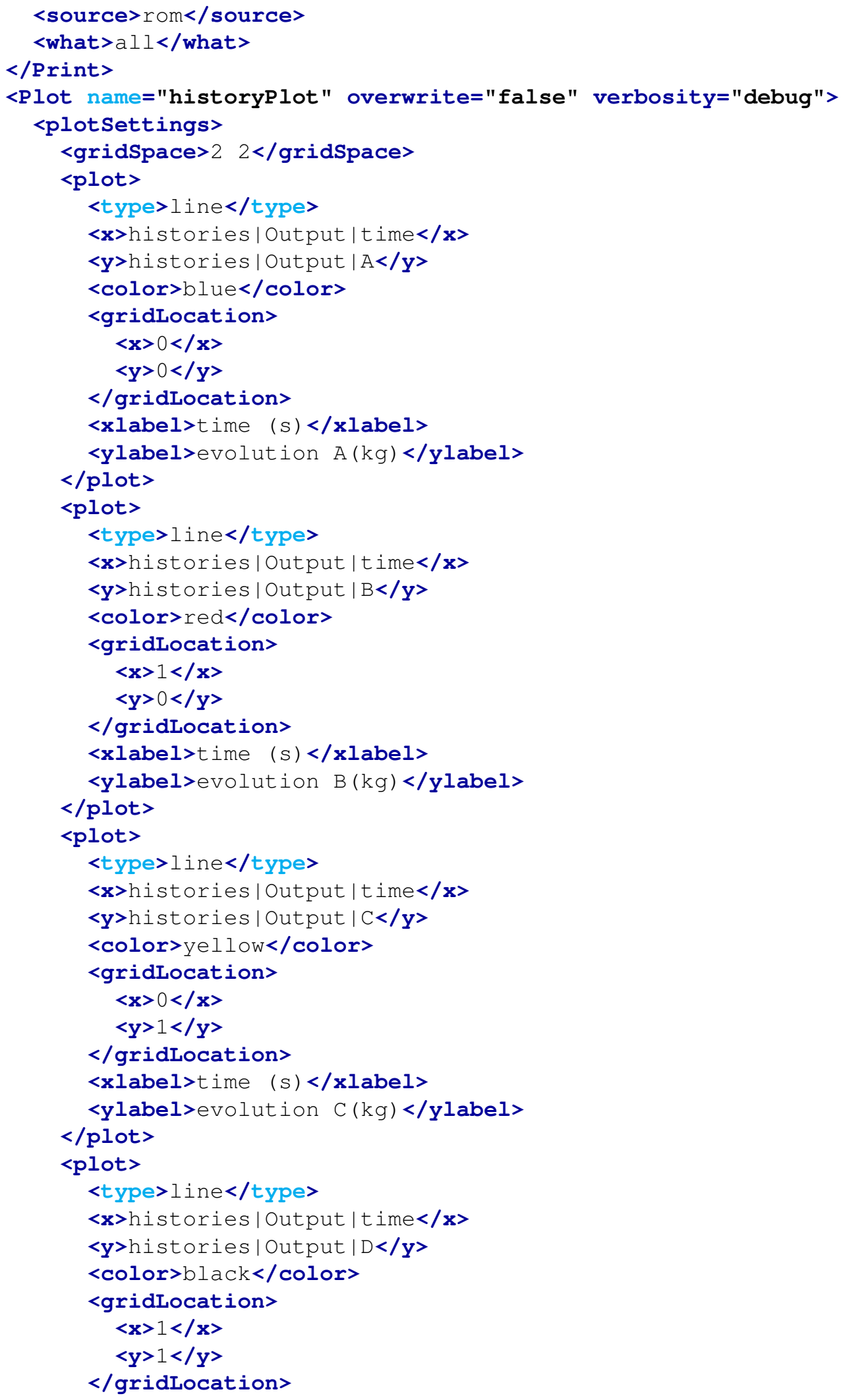




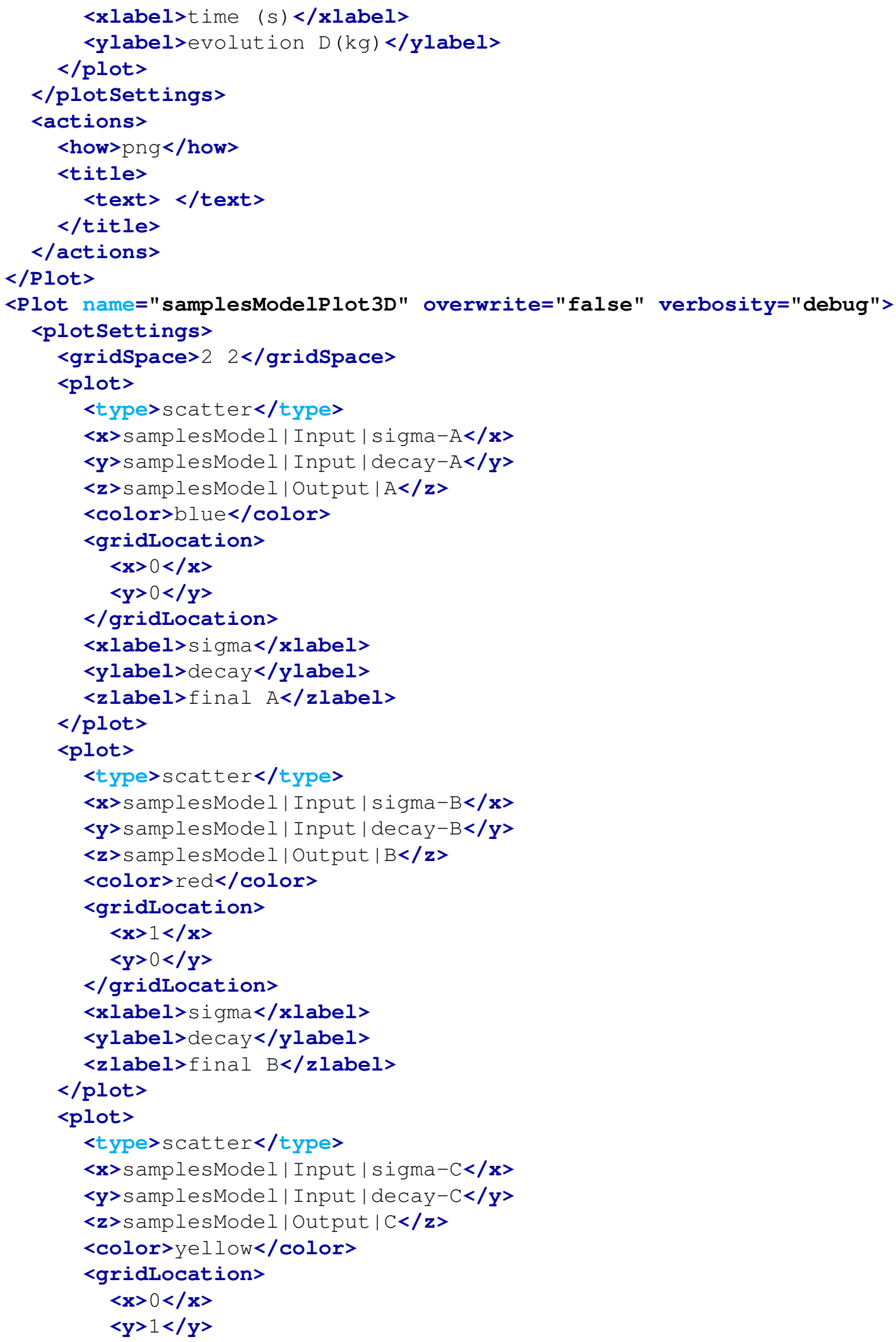




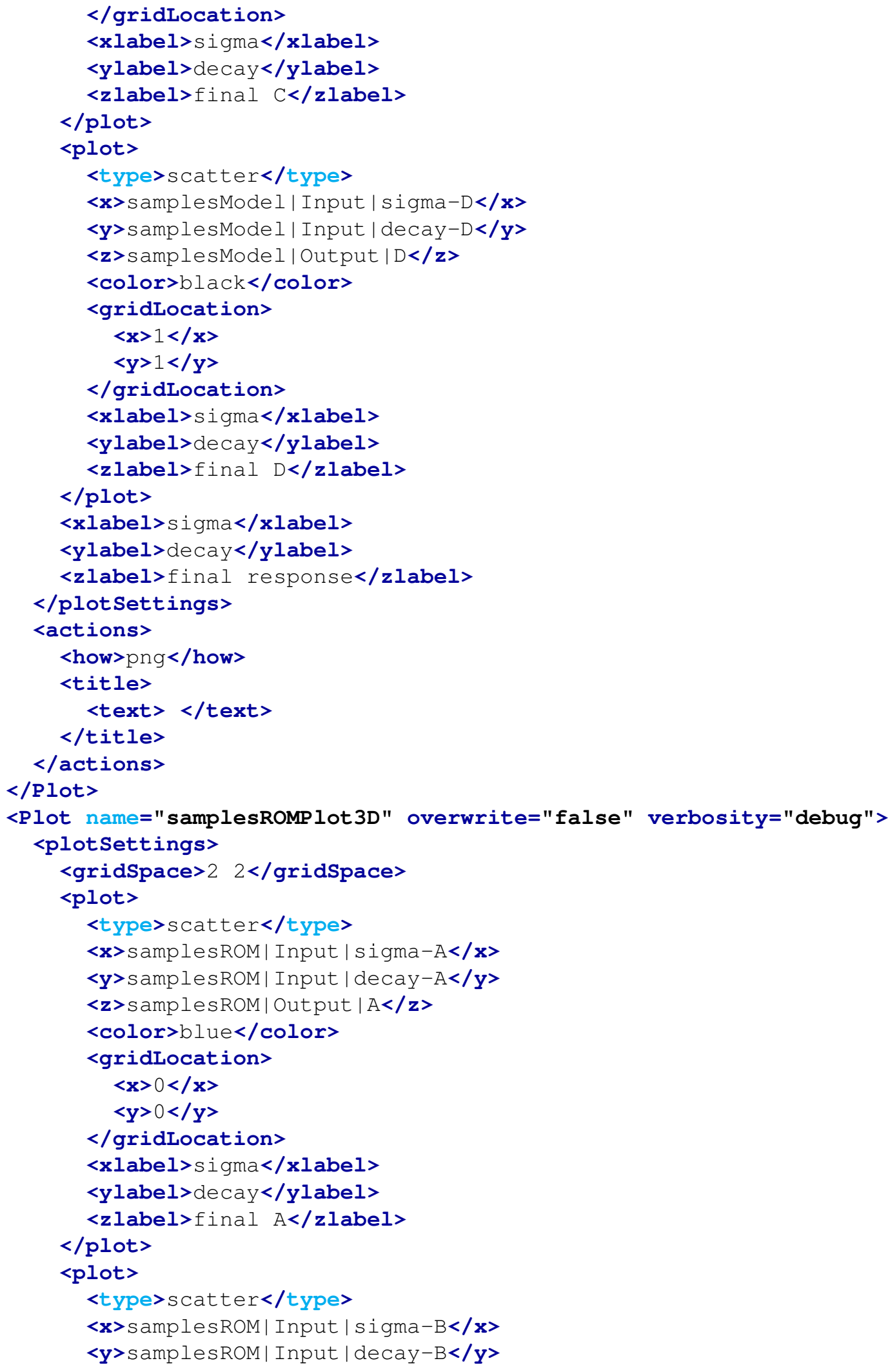




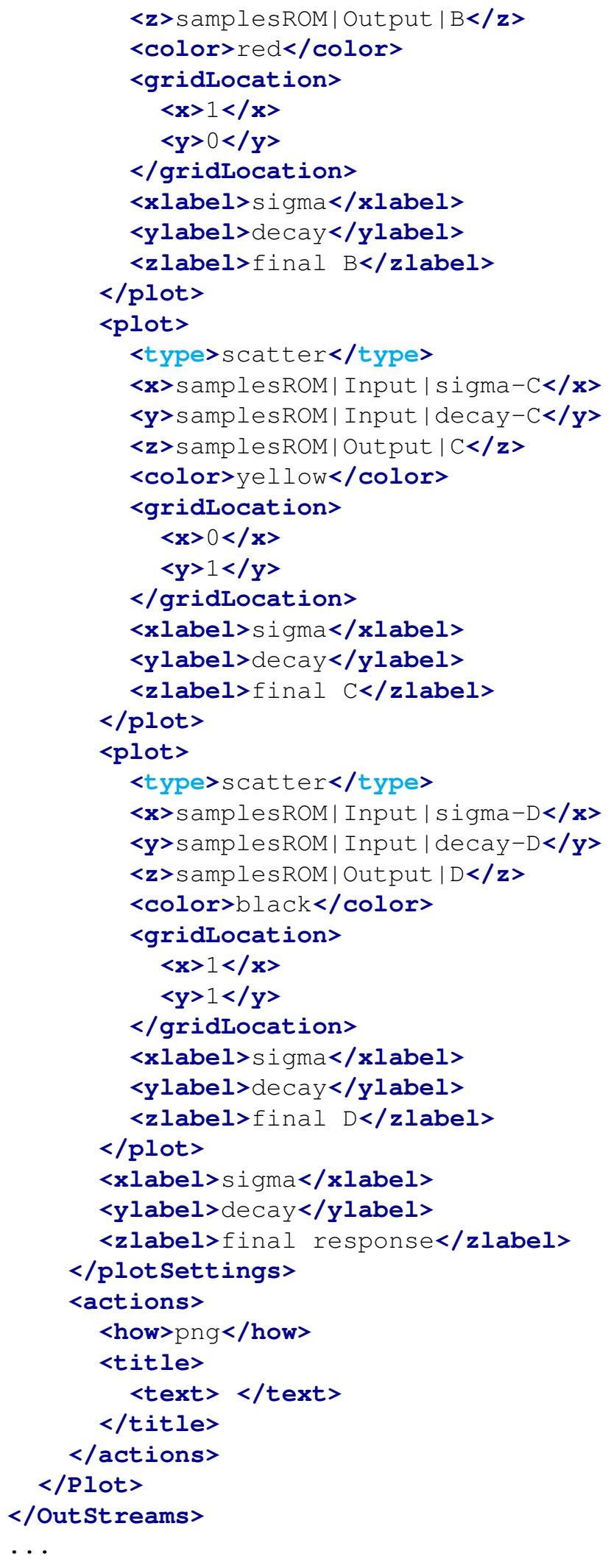


In this block, the following Out-Stream types are constructed:

- Print:

- named "samplesModel" connected with the DataObjects Entity "samplesModel" (<source>)

- named "samplesROM" connected with the DataObjects Entity "samplesROM" (<source>)

- named "histories" connected with the DataObjects Entity "histories" (<source>)

- named "rom_output" connected with the ROM Entity "rom" (<source>).

When these objects get used, all the information contained in the linked DataObjects are going to be exported in ether CSV files for DataObjects or XML files for ROMs (<type>).

- Plot:

- named "historyPlot" connected with the DataObjects Entity "histories". This plot will draw the time-dependent state of the variables $A, B, C, D$ with respect to the input variables sigma(s) and decay(s)

- named "samplesModelPlot3D" connected with the DataObjects Entity "samplesModel". This plot will draw the variables $A, B, C, D$ as Monte Carlo sampled on the Code.

- named "samplesROMPlot3D" connected with the DataObjects Entity "samplesROM". This plot will draw the variables $A, B, C, D$ as Monte Carlo sampled on the ROM.

As it can be noticed, both plots are of type SubPlot. Four plots are going to be placed in each of the figures.

\section{Steps:}

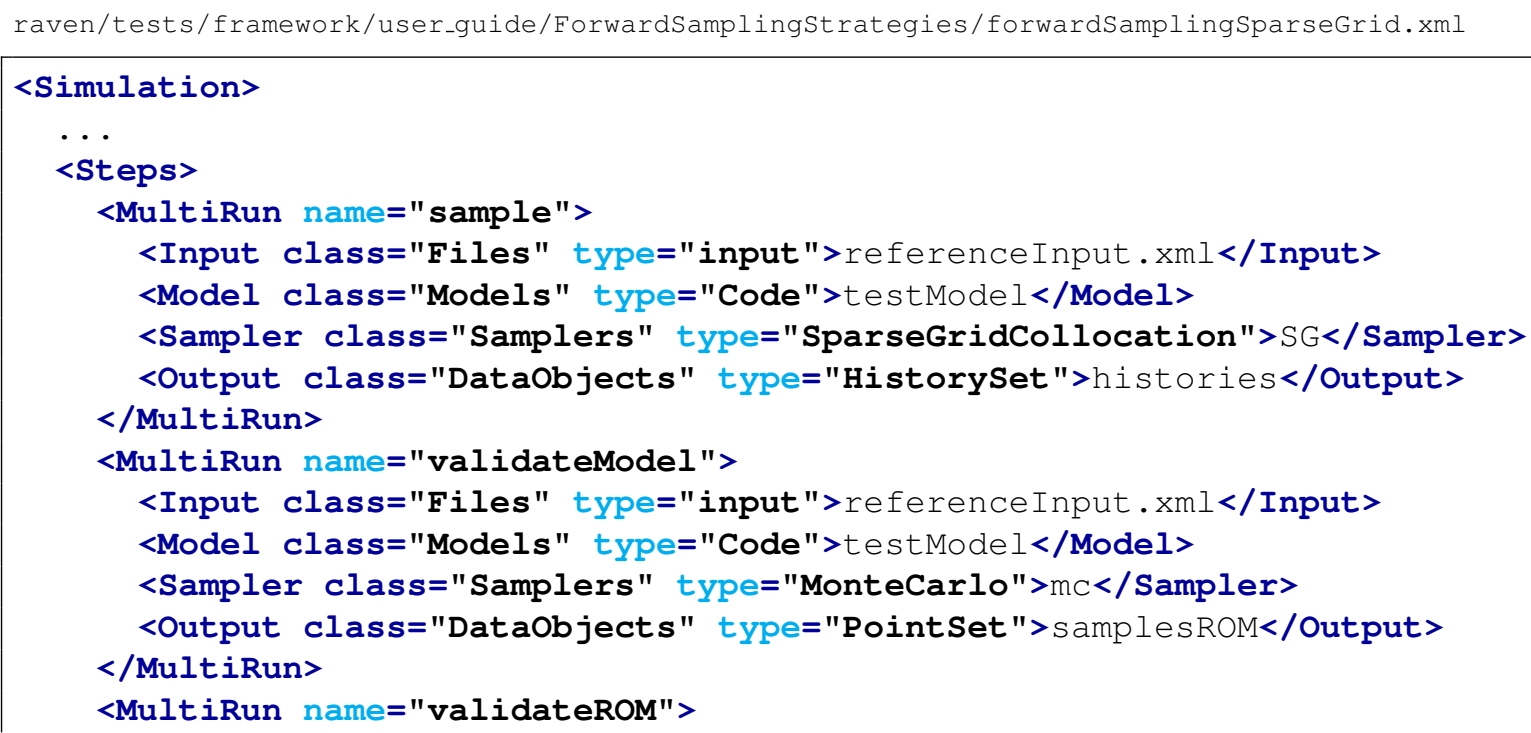




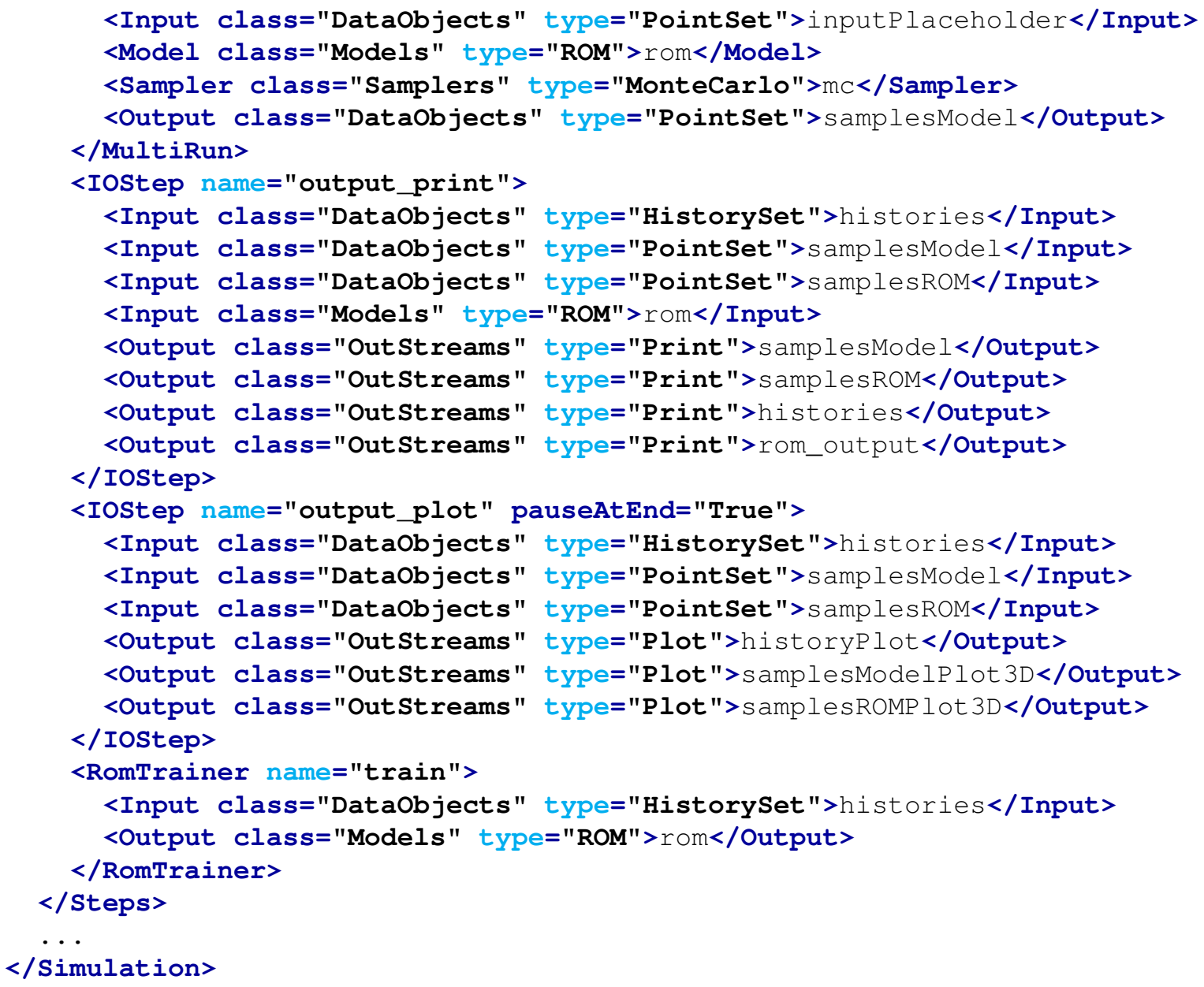

Finally, the previously defined Entities can be combined in the <steps > block. The following <steps> have been defined:

- <MultiRun> named "sample", used to run the training samples of the driven code and collect the outputs in the DataObjects. The <Sampler> is specified to communicate to the Step that the driven code needs to be sampled through the Sparse Grid Collocation sampling strategy;

- <RomTrainer> named "train", used to train (i.e., construct) the GaussPolynomial ROM. This step is essential if the user want to use the ROM in later steps;

- <MultiRun> named "sampleModel", used to run the Monte Carlo perturbed samples of the original Model for use in verification. The results are collected in the samplesModel DataObjects.

- <MultiRun> named "sampleROM", used to run the Monte Carlo perturbed samples of the previously constructed ROM for use in verificaiton. The results are collected in the samplesROM DataObjects. 

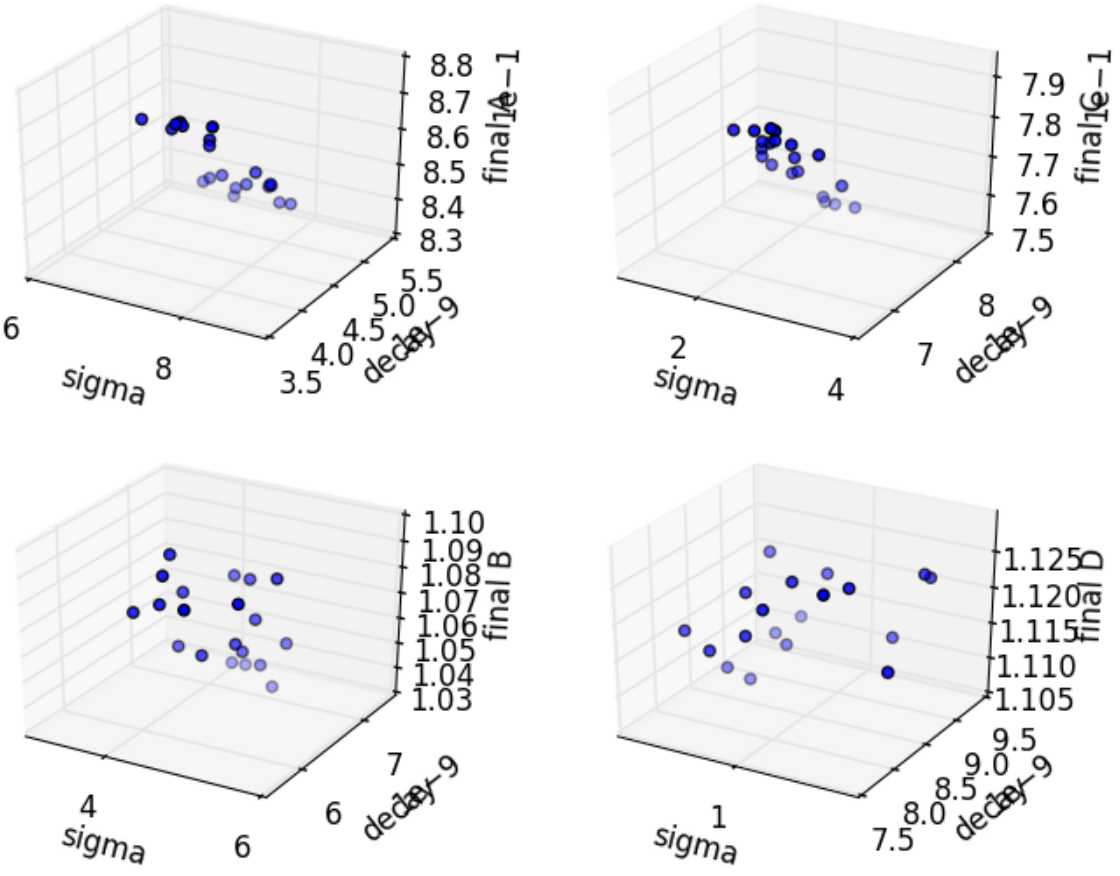

Figure 20. Plot of validation samples generated by Monte Carlo sampling on the Code for variables $A, B, C, D$.

- <IOStep> named "output_print", used to dump the "histories", "samplesModel" and "samplesROM" DataObjects Entity in a CSV file,

- <IOStep> named "output_plot", used to plot the data and store it in the PNG file and on the screen.

As previously mentioned, Figure 19 shows the evolution of the outputs $A, B, C, D$ under uncertainties. Figures 20 and ?? show the final responses of the sampling employed using the driven code and the ROM, respectively. As it can be seen, the constructed ROM can accurately represent the response of the driven code. This example shows the potential of reduced order modeling, in general, and of the GaussPolynomialRom, in particular. 

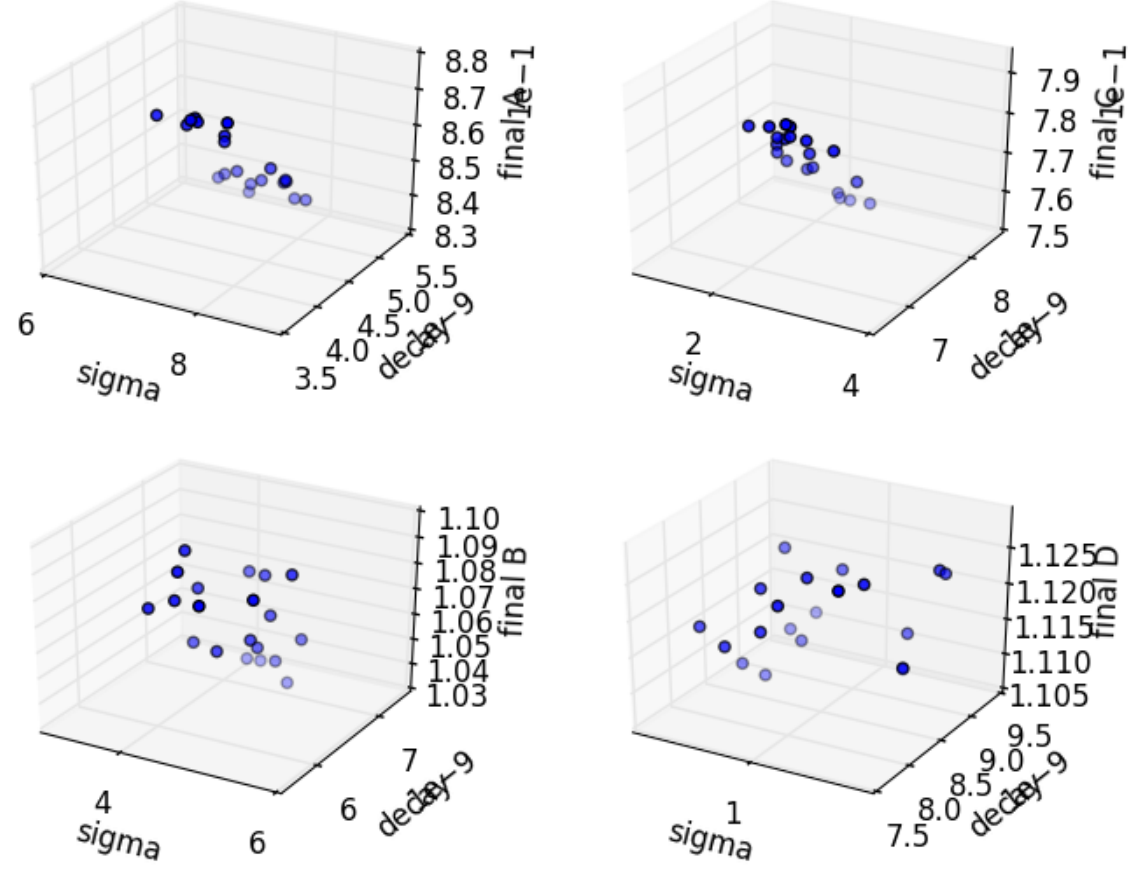

Figure 21. Plot of validation samples generated by Monte Carlo sampling on the ROM for variables $A, B, C, D$. 


\section{Adaptive Sampling Strategies}

Performing UQ and Dynamic PRA can be challenging from a computational point of view. The Forward sampling strategies reported in the previous Section can lead to a large number of unnecessary evaluations of the physical model leading to an unacceptable resource expenses (CPU time). In addition, the Forward methodologies are not designed to leverage the information content that is extractable from the simulations already concluded.

To overcome these limitations, in RAVEN several adaptive algorithms are available:

1. Limit Surface Search

2. Adaptive Dynamic Event Tree

3. Adaptive Hybrid Dynamic Event Tree

4. Adaptive Sparse Grid

5. Adaptive Sobol Decomposition.

In this Section, we will only show how to use the first algorithm.

\subsection{Limit Surface Search sampling through RAVEN}

The goal of this Section is to learn how to:

1. Set up a LS Search sampling for efficiently perturb a driven code

2. Use the LS Integral Post-processor for computing the probability of failure of the system subject to the same "goal" function

3. Plot the obtained LS.

In order to accomplish these tasks, the following RAVEN Entities (XML blocks in the input files) are defined:

\section{RunInfo:}

raven/tests/framework/user_guide/AdaptiveSamplingStrategies/adaptiveSamplingLSsearch.xml 


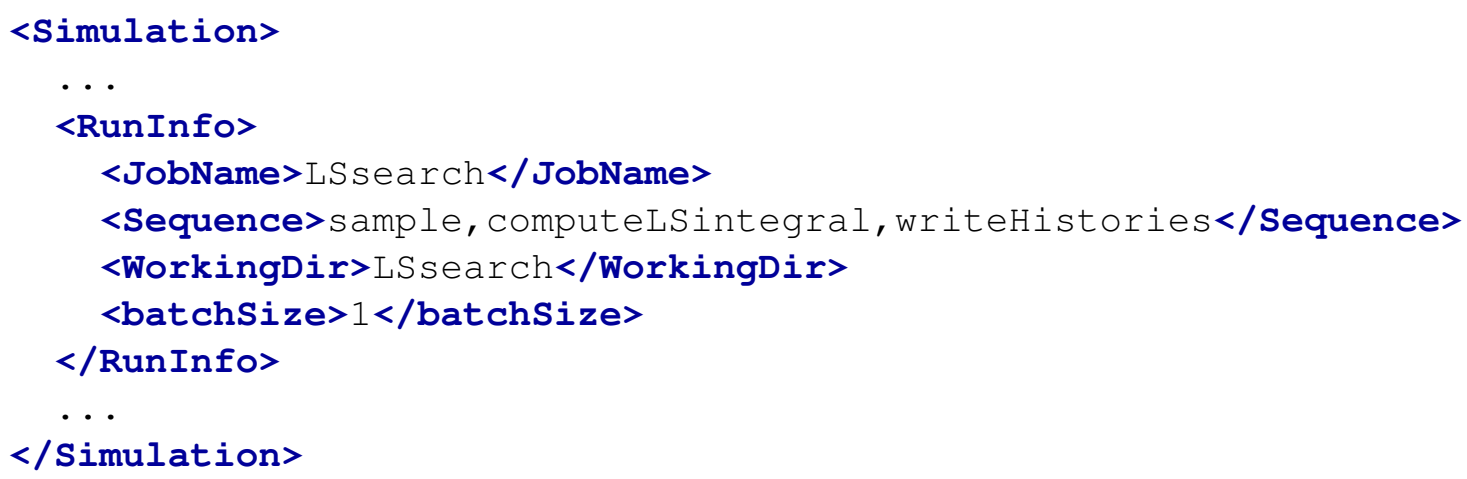

As shown in Section ??, the RunInfo Entity is intended to set up the analysis that the user wants to perform. In this specific case, three steps (<Sequence $>$ ) are sequentially run using eight processors (<batchsize>).

\section{Files:}

raven/tests/framework/user_guide/AdaptiveSamplingStrategies/adaptiveSamplingLSsearch.xml

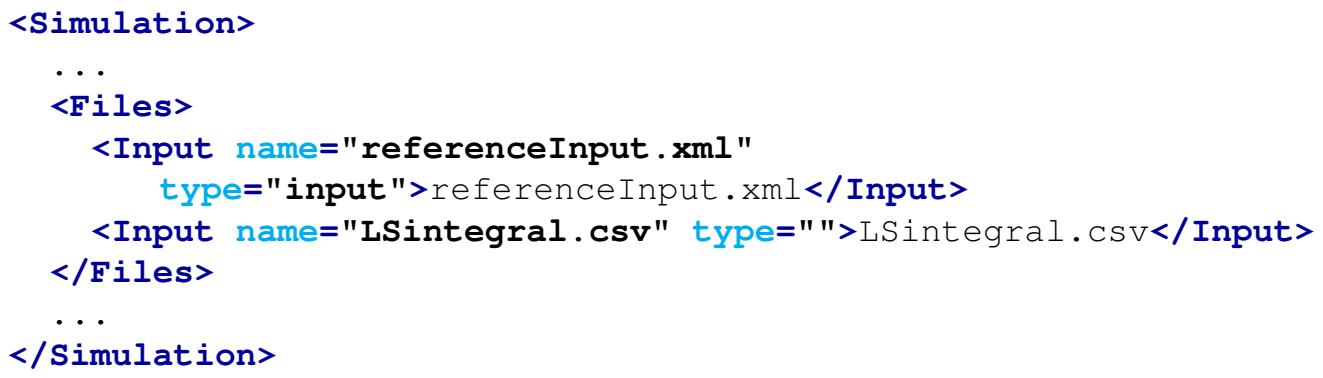

Since the driven code uses a single input file, in this Section the original input is placed. As detailed in the user manual the attribute name represents the alias that is going to be used in all the other input blocks in order to refer to this file.

In addition the output file used in <Sequence $>$ computeLSintegral is here inputted.

\section{Models:}

raven/tests/framework/user_guide/AdaptiveSamplingStrategies/adaptiveSamplingLSsearch.xml

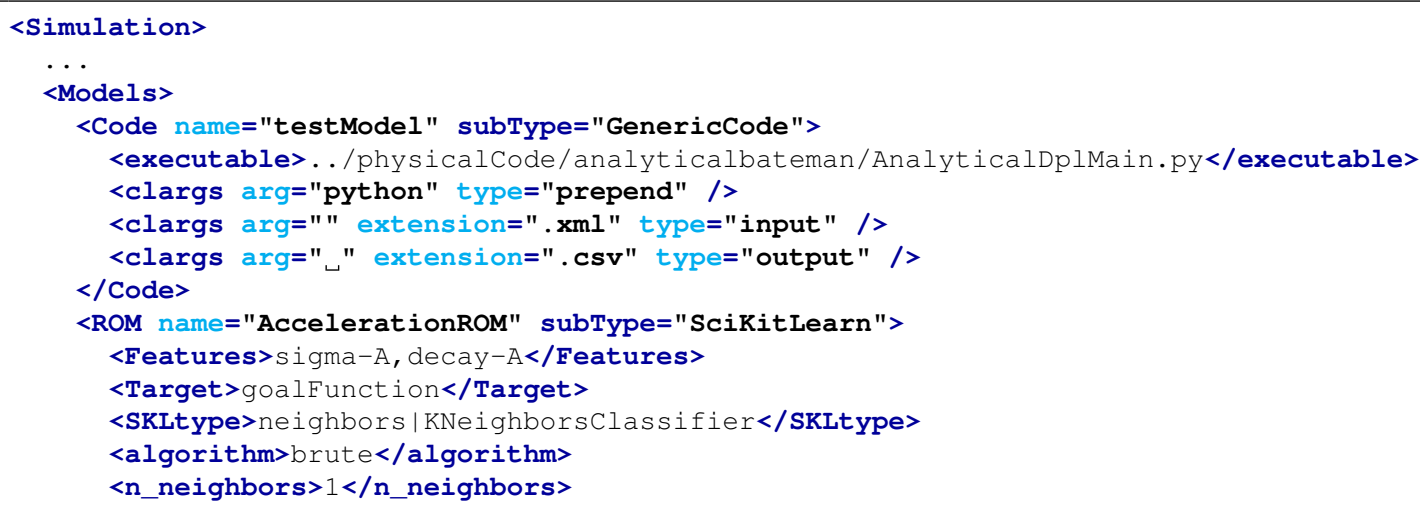




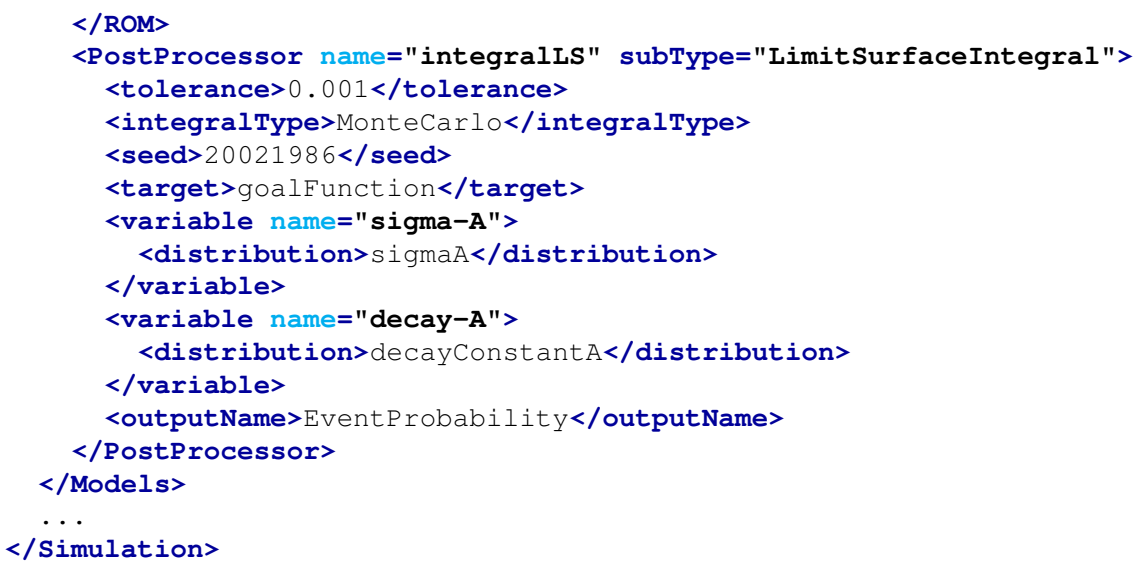

As mentioned above, the goal of this example is the employment of an efficient sampling strategy, having as goal the determination of the failure of a system.

In addition to the previously explained Code model, the ROM of type SciKitLearn is here specified. The ROM will be used in the adaptive sampling strategy LimitSurfaceSearch in order to accelerate the convergence of the method. As it can be seen, a nearest neighbor classifier is used, targeting only two uncertainties sigma - Aanddecay - A.

For the computation of the probability of failure (see the following), a Post-Processor (PP) of type LimitSurfaceIntegral is here specified.This PP performs an integral of the LS generated by the adaptive sampling technique.

\section{Distributions:}

raven/tests/framework/user_guide/AdaptiveSamplingStrategies/adaptiveSamplingLSsearch.xml

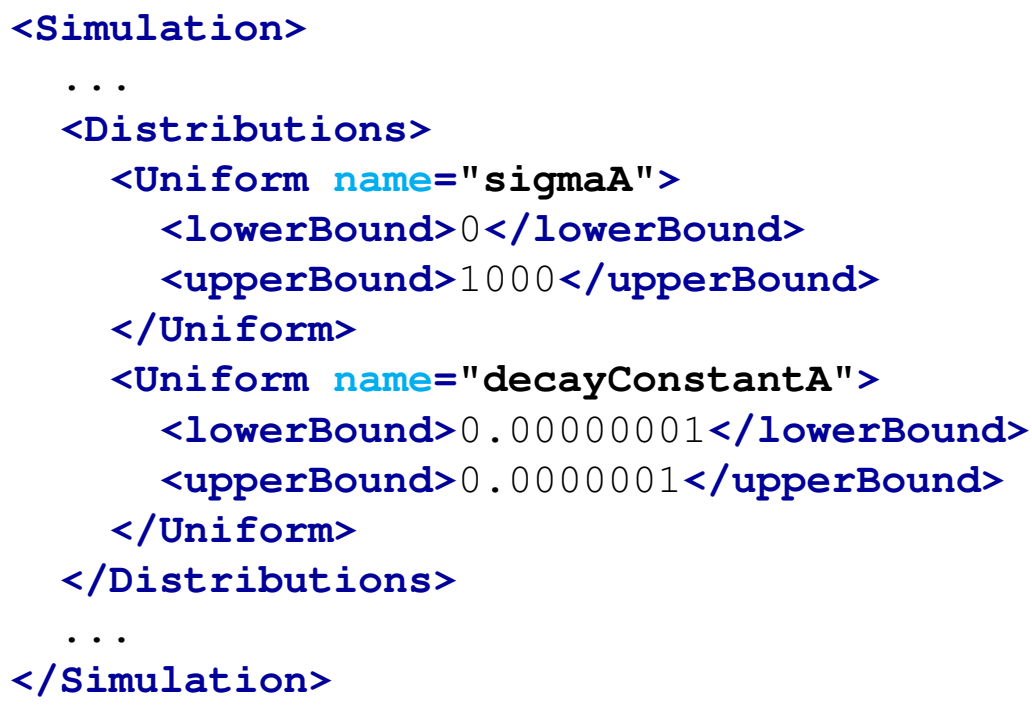

In the Distributions XML Section, the stochastic model for the uncertainties treated by the LS search sampling are reported. In this case two distributions are defined: 
- sigmaA $\sim \mathbb{U}(0,1000)$, used to model the uncertainty associated with the Model sigma- $A$

- decayConstant $A \sim \mathbb{U}(1 e-8,1 e-7)$, used to model the uncertainty associated with the Model decay-A.

\section{Samplers:}

raven/tests/framework/user_guide/AdaptiveSamplingStrategies/adaptiveSamplingLSsearch.xml

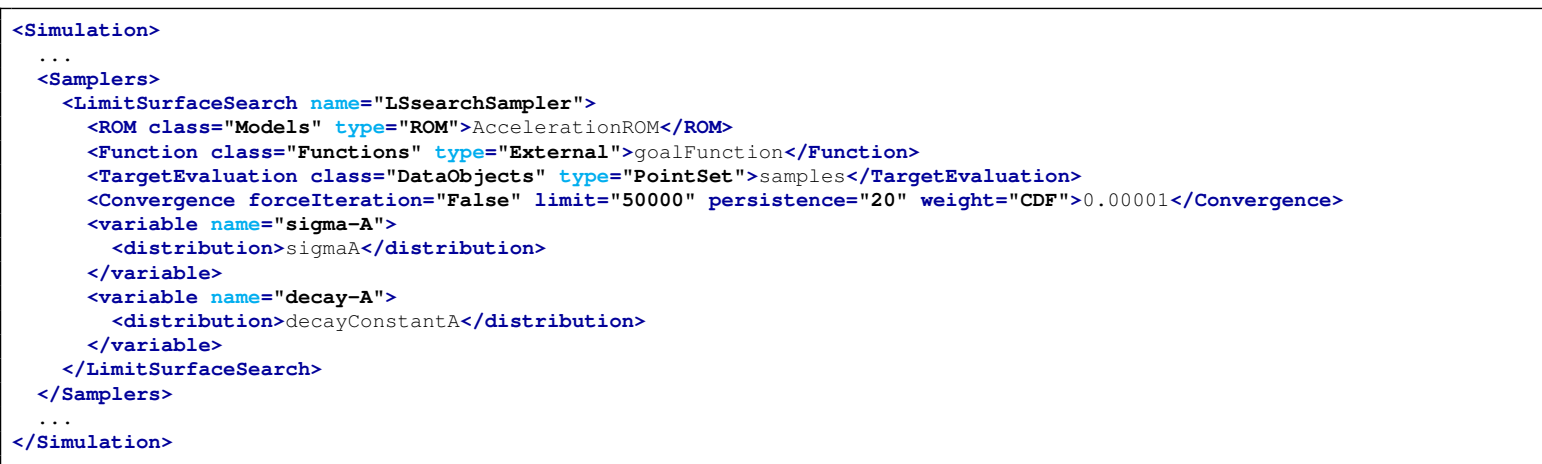

In order to employ the LS search sampling strategy, a <LimitSurfaceSearch $>$ node needs to be inputted. As it can be seen from above, each variable is associated to a different distribution defined in the $<$ Distributions $>$ block. In addition, the AccelerationROM $<$ ROM $>$ is inputted. As already mentioned, this ROM (of type classifier) is used to accelerate the convergence of the LS Search method. In addition, the goal function goalFunction and the samples are here reported.

For this example, a convergence criterion of $1.0 e-5$ is set. To reach such a confidence with a Monte-Carlo, millions of samples would be needed.

\section{Functions:}

raven/tests/framework/user_guide/AdaptiveSamplingStrategies/adaptiveSamplingLSsearch.xml

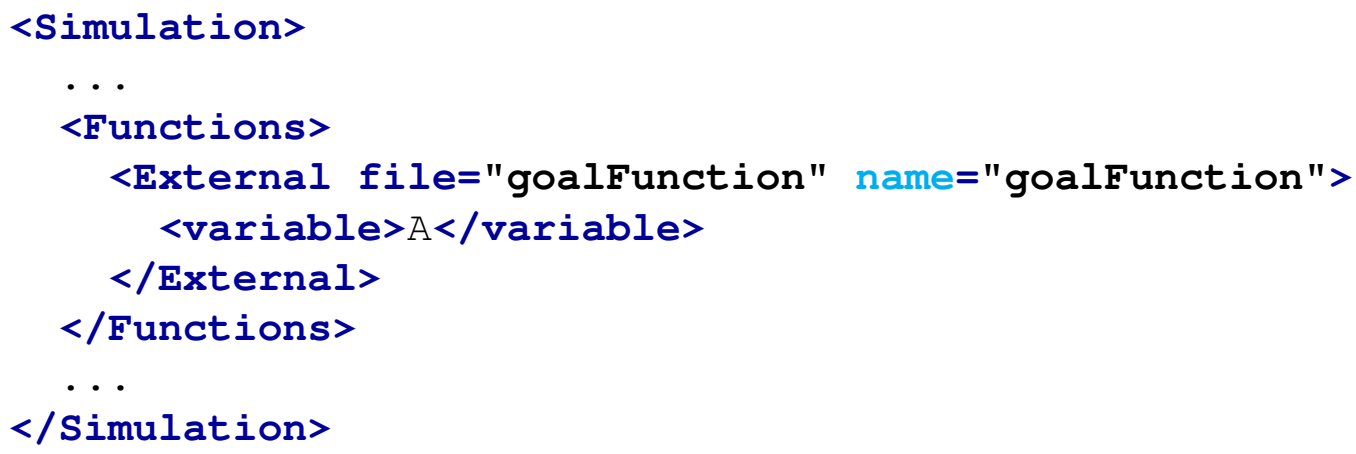

As already mentioned, the LS search sampling strategy uses a goal function in order to identify the regions of the uncertain space that are more informative. The goalFunction used for this example is reported below. As it can be seen, if the final response $A$ is $<=$ of 0.3 , the system is considered to be in a "safe" condition. 
def _residuumsign (self):

returnvalue $=1.0$

if self.A $<=0.3$ :

returnvalue $=-1.0$

return returnvalue

\section{DataObjects:}

raven/tests/framework/user_guide/AdaptiveSamplingStrategies/adaptiveSamplingLSsearch.xml

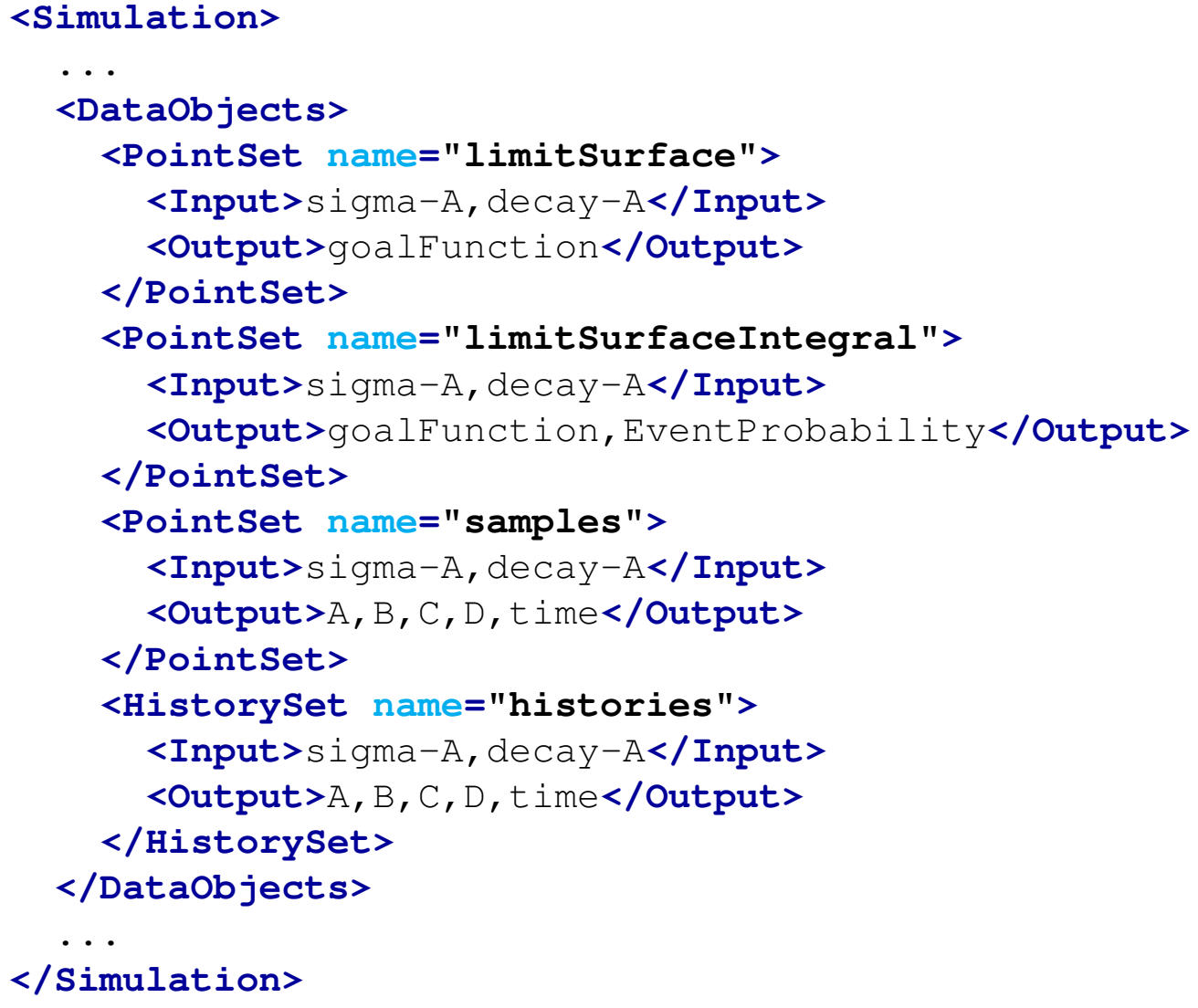

In this block, three DataObjects are defined: 1) PointSet named "samples" used to collect the final outcomes of the code, 2) HistorySet named "histories" in which the full time responses of the variables $A, B, C, D$ are going to be stored, 3) PointSet named "limitSurface" used to export the LS location (in the uncertain space) during the employment of the sampling strategy.

\section{OutStreams:}

raven/tests/framework/user_guide/AdaptiveSamplingStrategies/adaptiveSamplingLSsearch.xml

$<$ Simulation $>$

... 


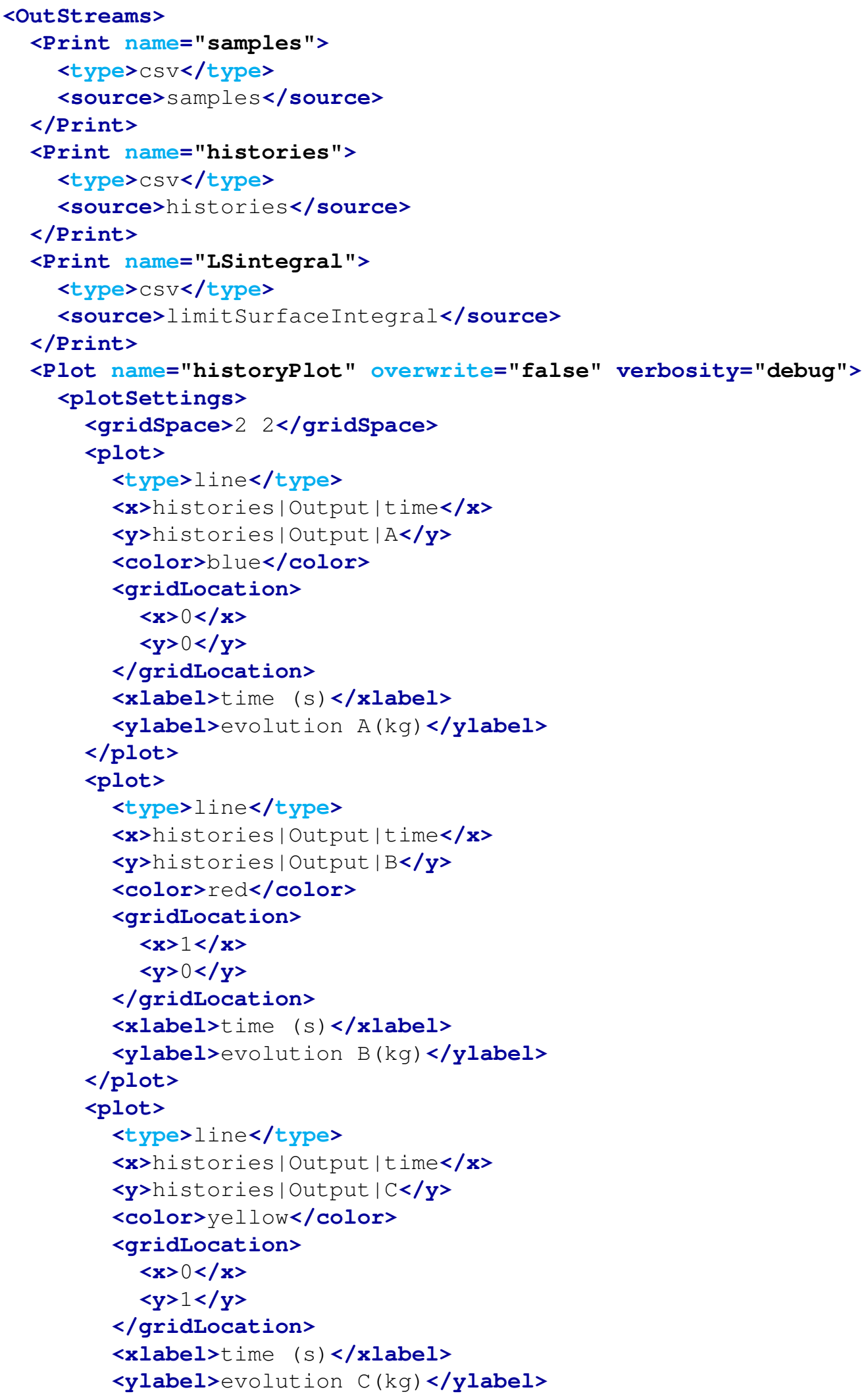




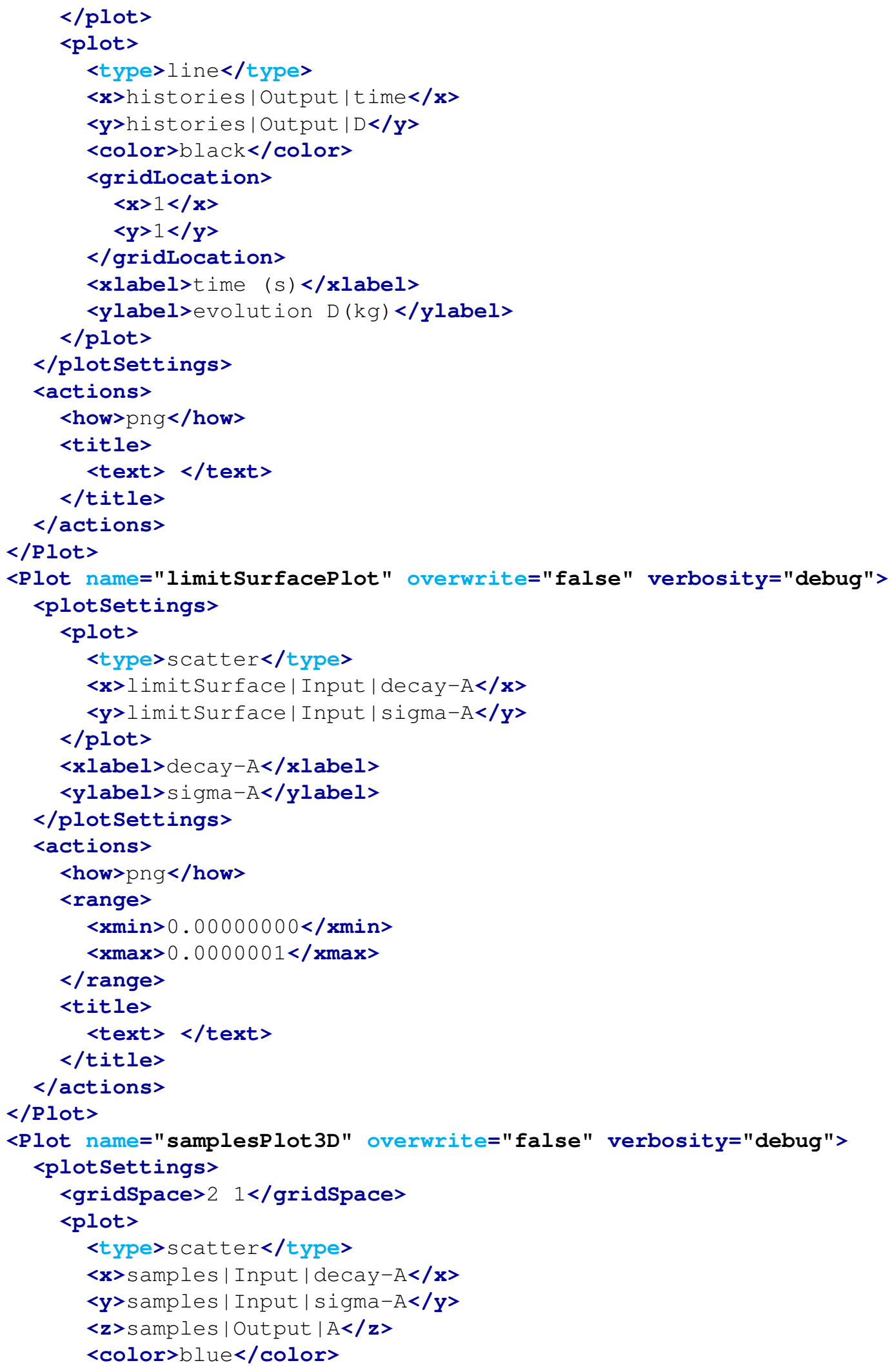




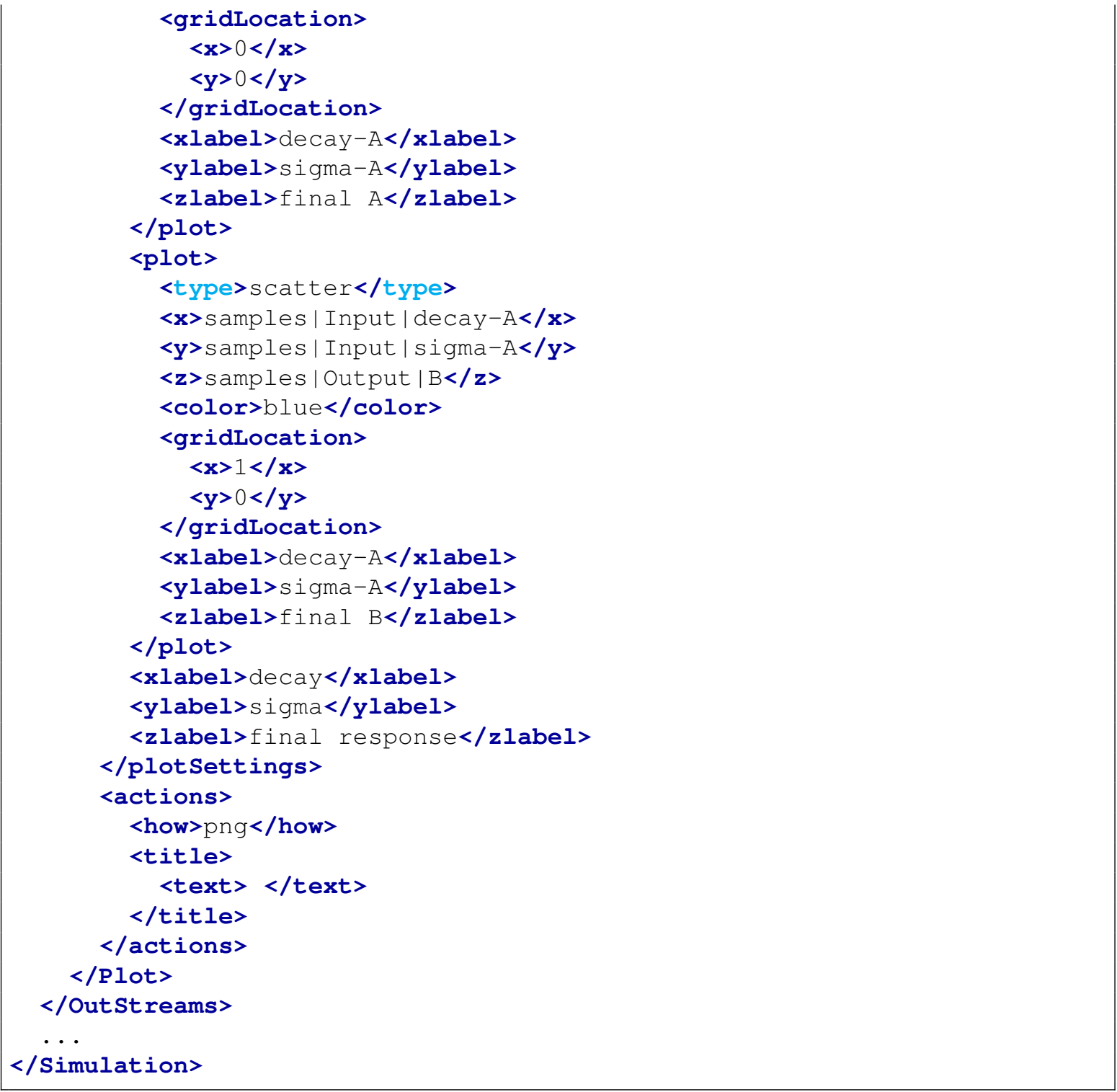

Several out streams are included in this workflow, two for printing and three for plotting:

- "samples", which writes the validation sample contents of the ' samples' PointSet DataObject to a CSV file,

- "histories", which writes the sampling contents of the 'histories' HistorySet DataObject to a set of connected CSV files,

- "historyPlot", which plots the evolution of the samples taken,

- "limitSurfacePlot", which plots the limit surface discovered by the PostProcessor,

- "samplesPlot3D", which plots the final state of the samples taken against the figures of merit. 
The plots demonstrate how visualization of three-dimensional data, time-dependent data, and limit surfaces can be realized using RAVEN.
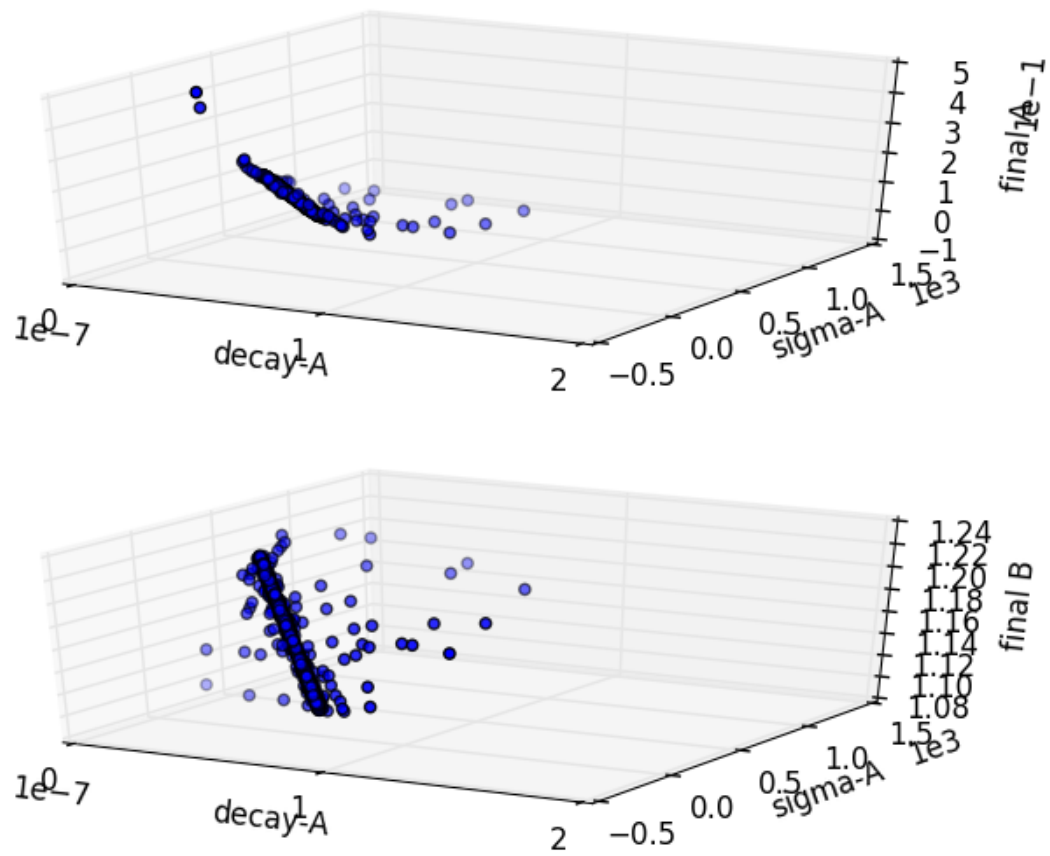

Figure 22. Plot of the samples generated by the LS search sampling for variables $A, B$.

\section{Steps:}

raven/tests/framework/user_guide/AdaptiveSamplingStrategies/adaptiveSamplingLSsearch.xml

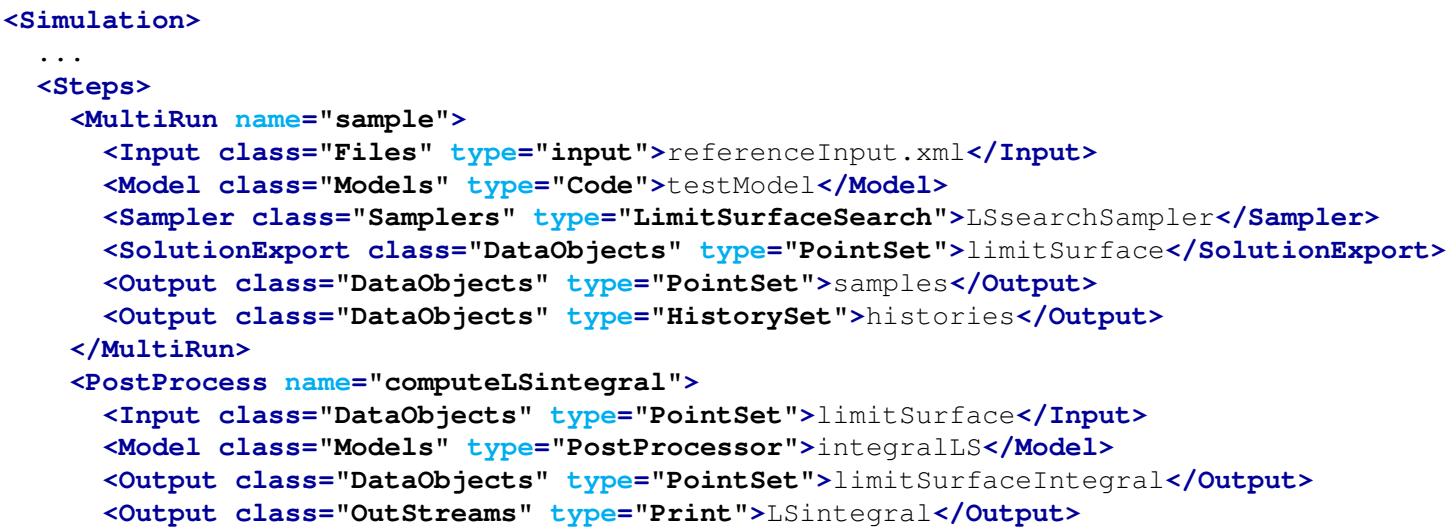



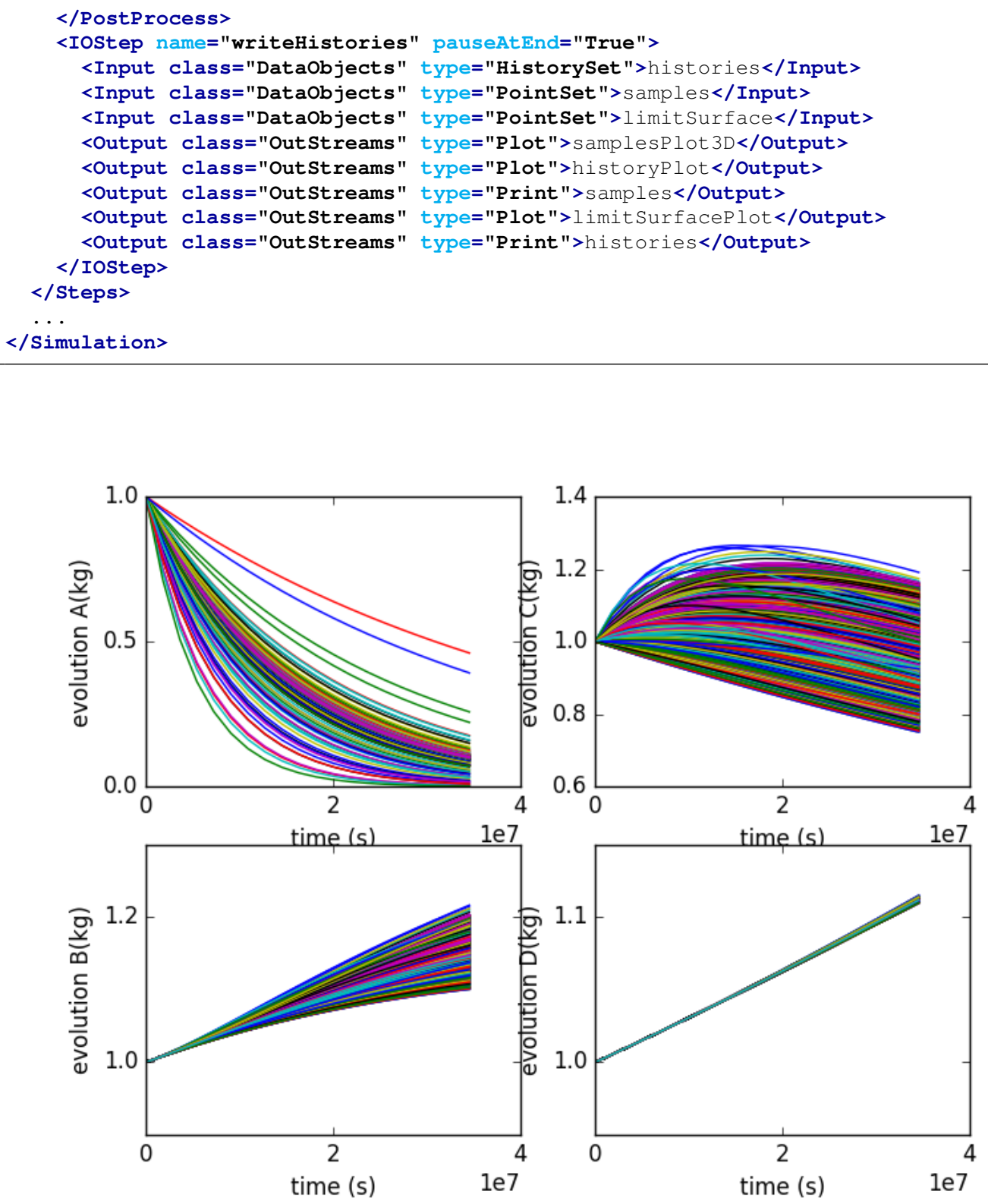

Figure 23. Plot of the histories generated by the LS search method for variables $A, B, C, D$.

Finally, all the previously defined Entities can be combined in the <Steps > block. As inferable, three <Steps> have been inputted:

- <MultiRun> named "sample", used to run the multiple instances of the driven code and collect the outputs in the two DataObjects. As it can be seen, the <Sampler $>$ is 
inputted to communicate to the Step that the driven code needs to be perturbed through the LS search sampling strategy;

- <PostProcess> named "computeLSintegral", used to compute the probability of failure of the system based on the LS generated employing the LS search strategy. This probability is computed integrating the LS with a Monte-Carlo method.

- <IOStep> named "writeHistories", used to 1) export the "histories" and "samples" DataObjects Entity in a CSV file and 2) plot the data and the Limit Surface in PNG files and on the screen.

Figure 23 shows the evolution of the outputs $A, B, C, D$ under uncertainties. Figure 22 shows the final responses of $A a n d B$ of the sampling employed using the driven code. Figure 24 shows the limit surface for this particular example. Only 367 samples were needed in order to reach the full convergence.

The integration of the LS determines a probability of failure of $3.45 e-2$.

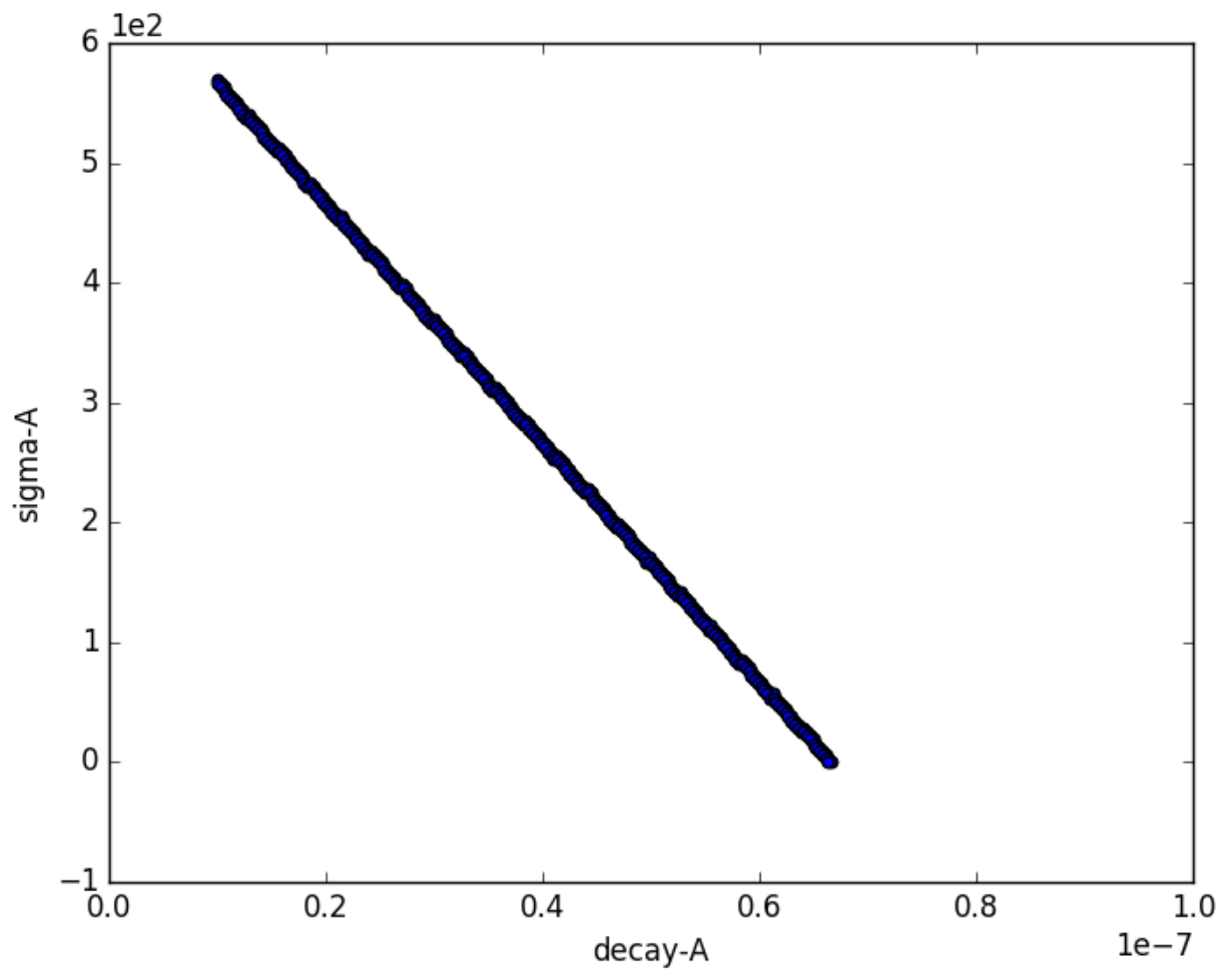

Figure 24. Limit Surface generated by the LS search method. 


\section{Sampling from Restart}

In some instances, there are existing solutions stored that are useful to a new sampling calculation. For example, if a Monte Carlo run collects 1000 runs, then later the user decides to expand to 1500 runs, the original 1000 should not be wasted. In this case, it is desirable to restart sampling.

All <Sampler> entities in RAVEN accept the <Restart> node, which allows the user to provide a $<$ DataObject $>$ from which sampling can draw. The way each sampler interacts with this restart data is dependent on the sampling strategy.

Random sampling strategies, such as the <MonteCarlo> and <Stratified> samplers, increment the random number generator by the number of samples in the restart data, then continue sampling as normal.

Grid-based sampling strategies, such as <Grid>, <SparseGridCollocation>, and <Sobol>, require specific sampling points. As each required point in the input space is determined, the sampler will check the restart data for a match. If a match is found, the corresponding output values are used instead of sampling the <Model $>$ for that point in the input space. In order to determine a match, all of the values in the restart point must be within a relative tolerance of the corresponding point required by the sampler. While RAVEN has a default tolerance of 1e-15, the user can adjust this tolerance using the <restartNode> node in the <Sampler $>$ block.

In order to demonstrate this restart method, we include here an example of restarting a <Grid> sampler. This example runs a simple example Python code from the command line using the <GenericCode> interface. Within the run the following steps occur:

1. A grid is sampled that includes only the endpoints in each dimension.

2. The results of the first sampling are written to file.

3. The results in the CSV are read back in to a new <DataObject $>$ called ' restart'.

4. A second, more dense grid is sampled that requires the points of the first sampling, plus several more. The results are added both to the original $<$ DataObject $>$ as well as a new one, for demonstration purposes.

5. The results of only the new sampling can be written to CSV because we added the second data object in the last step.

6. Lastly, the complete <DataObject $>$ is written to file, including both the original and more dense sampling.

By looking at the contents of GRIDdump1.CSV, GRIDdump2. CSV, and GRIDdump3. CSV, the progressive construction of the data object becomes clear. GRIDdump1. CSV contains only 
a few samples corresponding to the endpoints of the distributions. GRIDdump 3. CSV contains all the points necessary to include the midpoints of the distributions as well as the endpoints. GRIDdump2 . CSV contains only those points that were not already obtained in the first sampling, but still needed for the more dense sampling.

raven/tests/framework/Samplers/Restart/Truncated/grid.xml

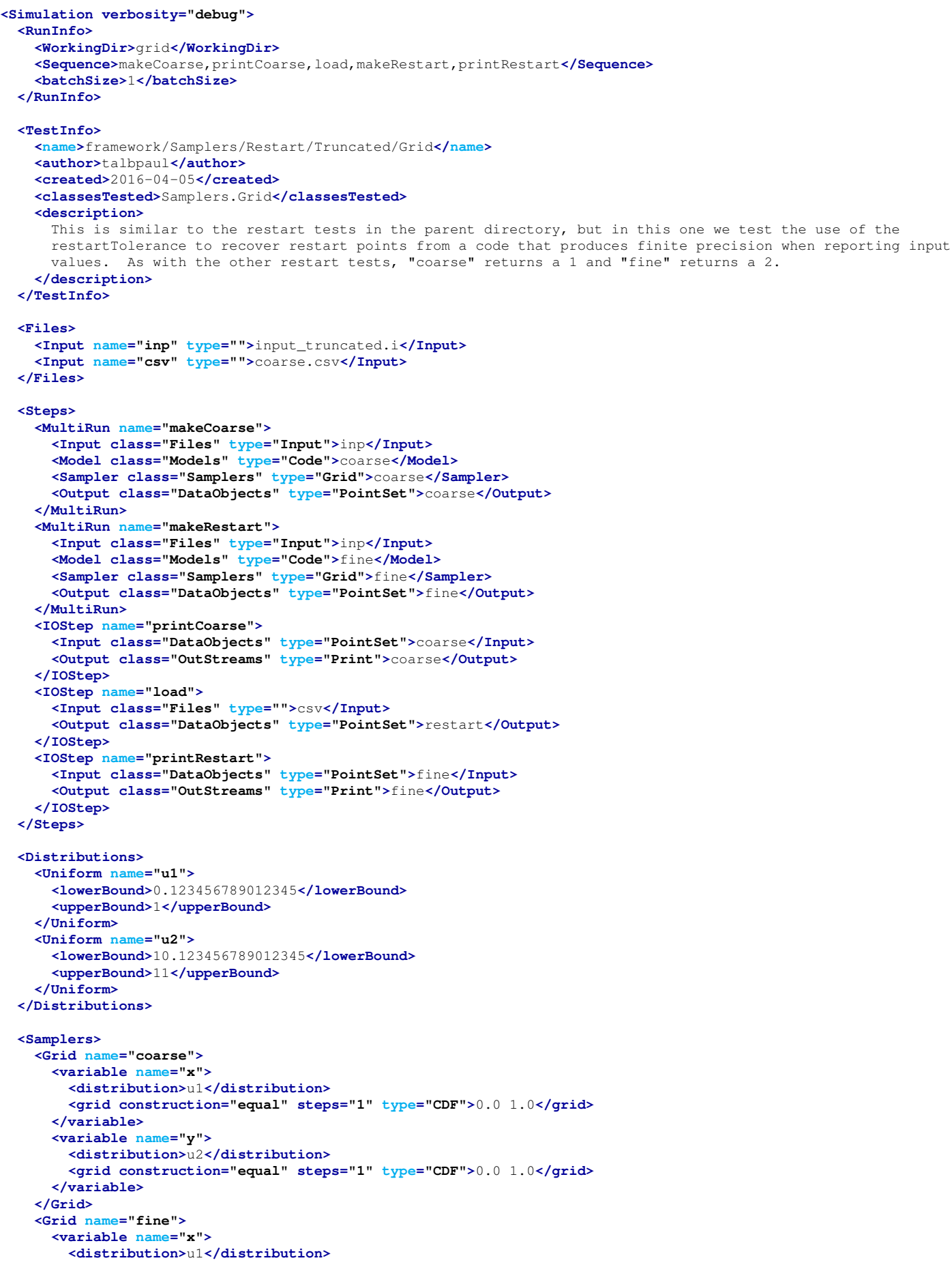




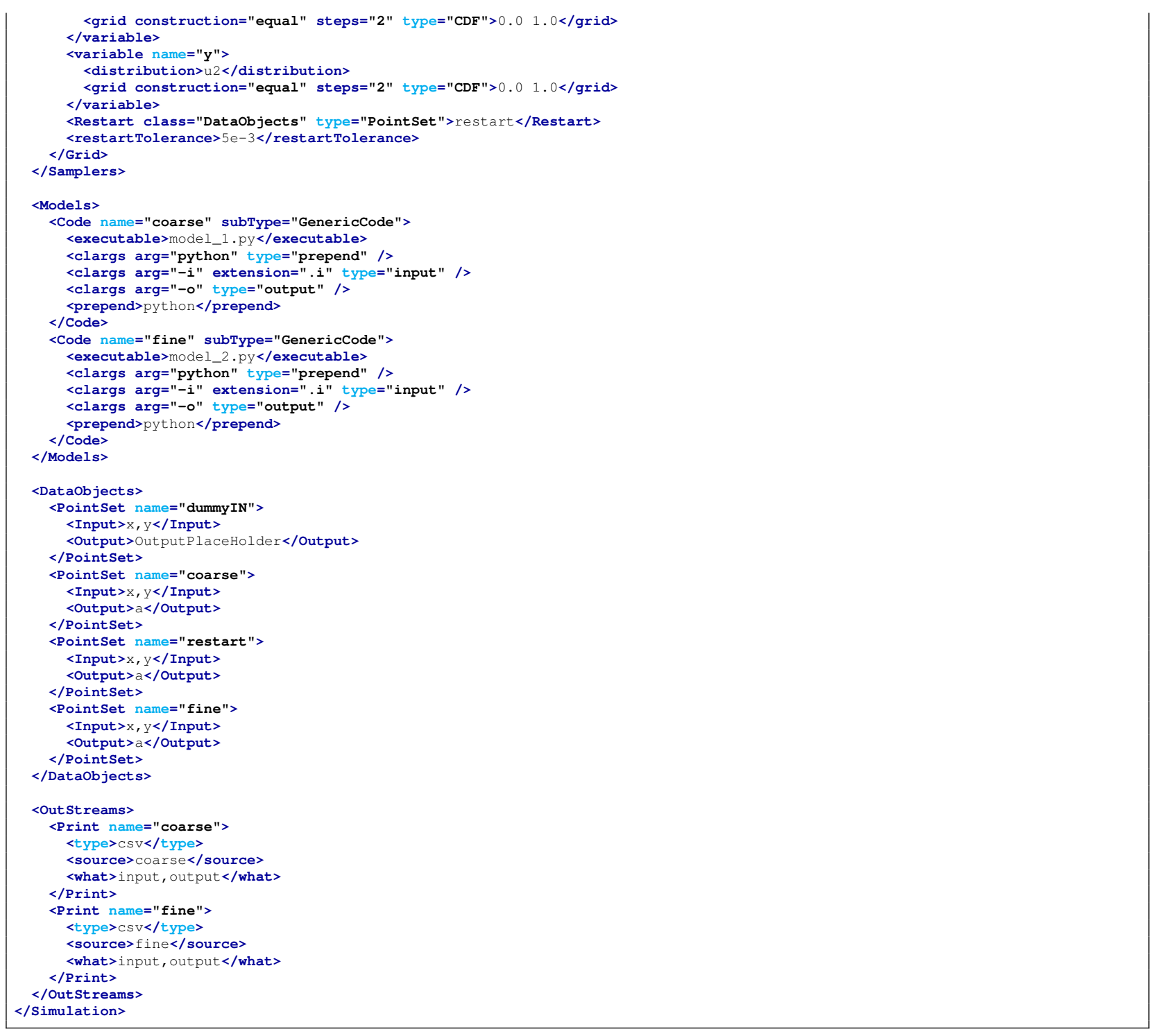




\section{Reduced Order Modeling through RAVEN}

The development of high-fidelity codes, for thermal-hydraulic systems and integrated multi-physics, has undergone a significant acceleration in the last years. Multi-physics codes simulate multiple physical models or multiple simultaneous physical phenomena, in a integrated solving environment. Multi-physics typically solves coupled systems of partial differential equations, generally characterized by several different geometrical and time scales.

The new multi-physics codes are characterized by remarkable improvements in the approximation of physics (high approximation order and reduced use of empirical correlations). This greater fidelity is generally accompanied by a greater computational effort (calculation time increased). This peculiarity is an obstacle in the application of computational techniques of quantification of uncertainty and risk associated with the operation of particular industrial plant (e.g., a nuclear reactor).

A solution to this problem is represented by the usage of highly effective sampling strategies. Sometimes also these approaches is not enough in order to perform a comprehensive UQ and PRA analysis. In these cases the help of reduced order modeling is essential.

RAVEN has support of several different ROMs, such as:

\section{Nearest Neighbors approaches}

\section{Support Vector Machines}

\section{Inverse Weight regressors}

4. Spline regressors, etc.

A ROM, also known a surrogate model, is a mathematical representation of a system, used to predict a FOM of a physical system.

The "training" is a process of setting the internal parameters of the ROM from a set of samples generated the physical model, .e., the high-fidelity simulator (RELAP-7, RELAP5 3D, PHISICS, etc.),

Two characteristics of these models are generally assumed (even if exceptions are possible):

1. The higher the number of realizations in the training sets, the higher is the accuracy of the prediction performed by the ROM is. This statement is true for most of the cases, although some ROMs might be subject to the over-fitting issues. The over-fitting phenomenon is not analyzed in this thesis, since its occurrence highly depends on the algorithm type, and, hence, the problem needs to be analyzed for all the large number of ROM types available; 


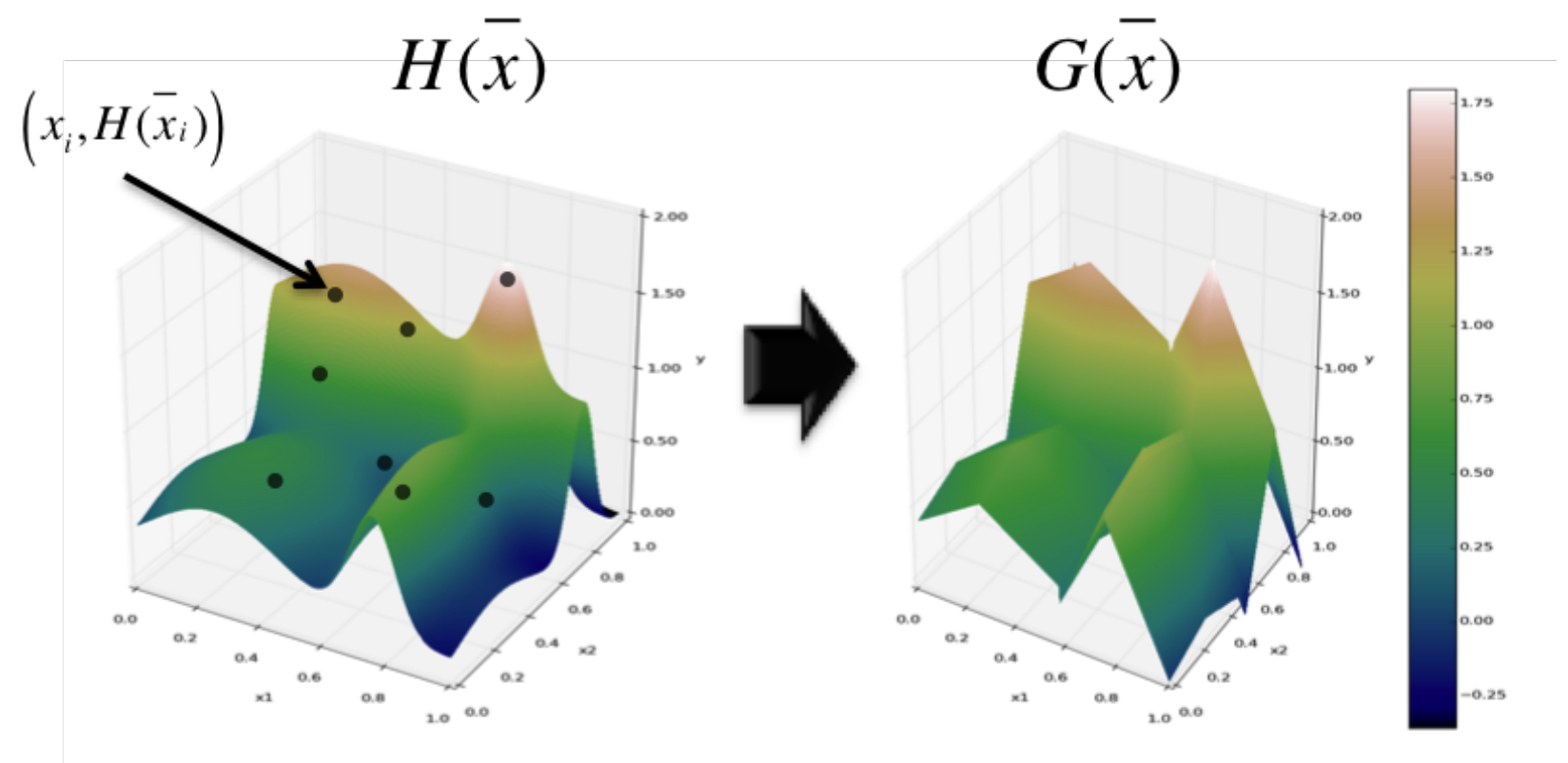

Figure 25. Example of reduced order model representation of physical system (regression).

2. The smaller the size of the input (uncertain) domain with respect to the variability of the system response, the more likely the ROM is able to represent the system response space.

The goals of this section are about learning how to:

1. Set up a sampling strategy to construct multiple ROMs, perturbing a driven code

2. Train the different ROMs with the data-set obtained by the applied sampling strategy;

3. Use the same sampling strategy, perturbing the ROMs

4. Plot the responses of the driven code and ROMs, respectively.

In order to accomplish these tasks, the following RAVEN Entities (XML blocks in the input files) need to be defined:

\section{RunInfo:}

raven/tests/framework/user_guide/ReducedorderModeling/reducedorderModeling.xml 


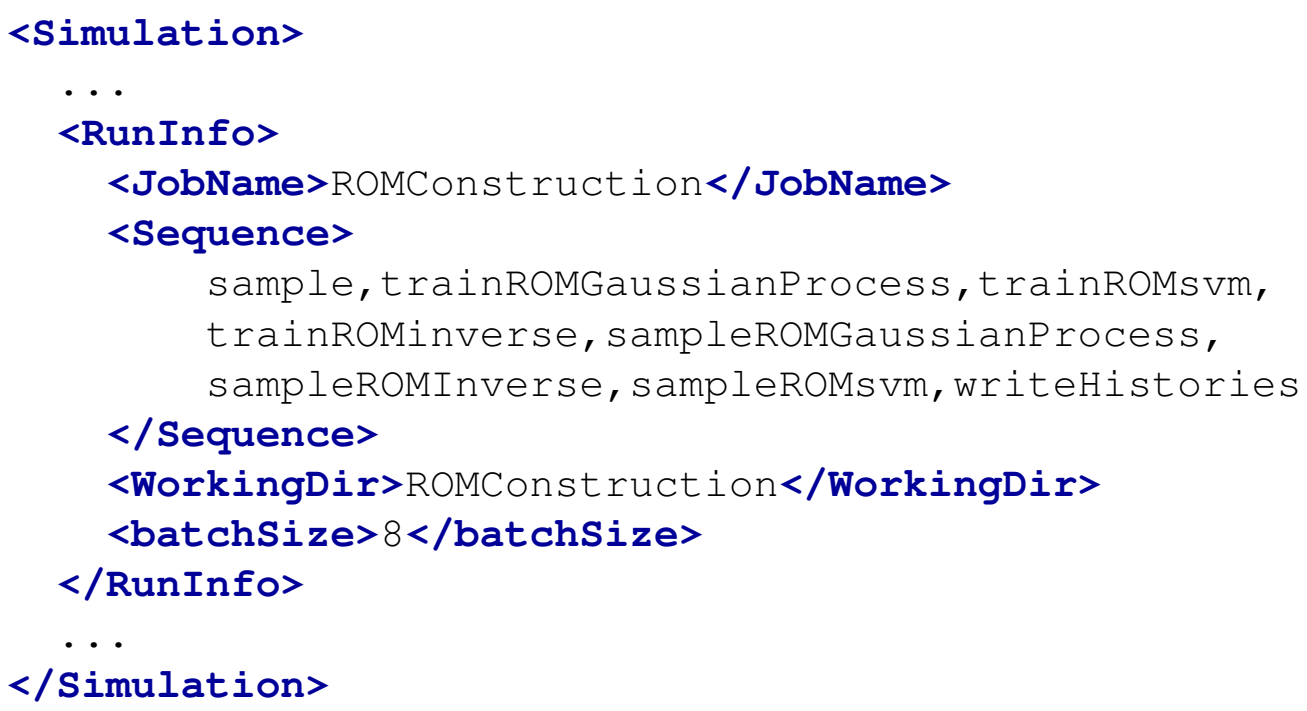

As in the other examples, the the RunInfo Entity is intended to set up the analysis sequence that needs to be performed. In this specific case, eight steps (<Sequence>) are going to be sequentially run using eight processors (<batchsize $\rangle$ ).

In the first step, the original physical model is going to be sampled. The obtained results are going to be used to train three different ROMs. These ROMs are sampled by the same strategy used in the first step in order to compare the ROMs' responses with the ones coming from the original physical model.

\section{Files:}

raven/tests/framework/user_guide/ReducedorderModeling/reducedorderModeling.xml

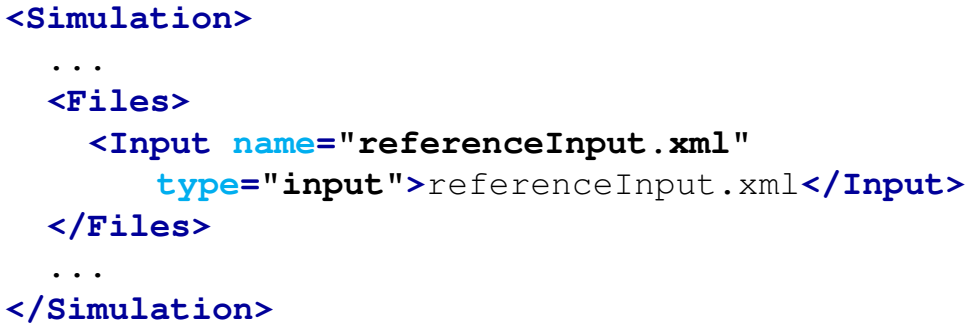

Since the driven code uses a single input file, the original input is placed in this section. As detailed in the user manual the attribute name represents the alias that is going to be used in all the other input blocks in order to refer to this file.

\section{Models:}

raven/tests/framework/user_guide/ReducedOrderModeling/reducedorderModeling.xml

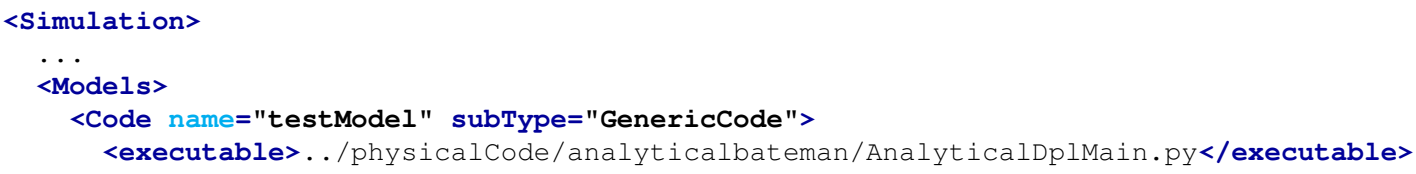




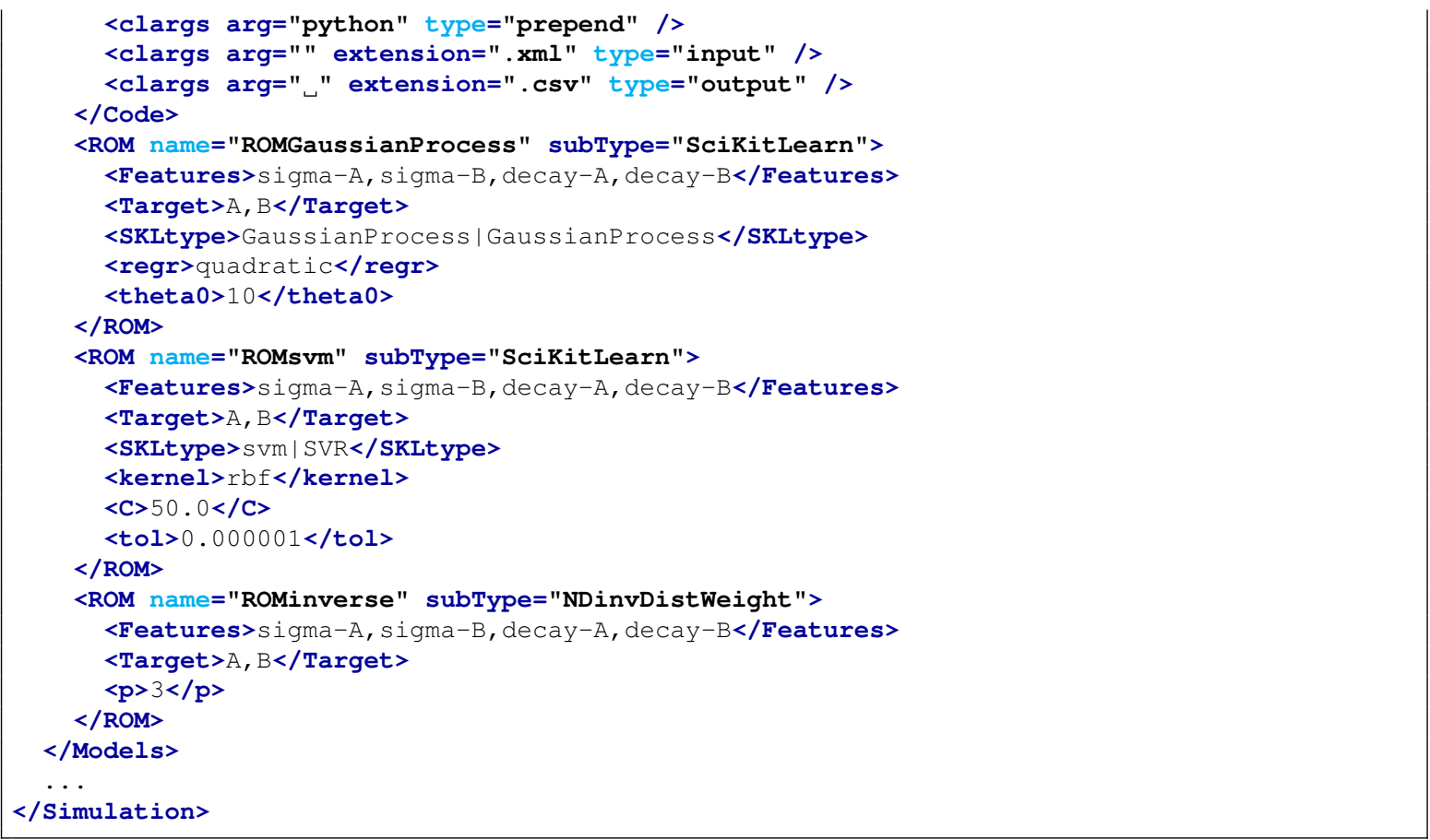

As mentioned above, the goal of this example is the employment of a sampling strategy in order to construct multiple types of ROMs.

Indeed, in addition to the previously explained Code model, three different ROMs (GP, SVM and IDW) are here specified. The ROMs will be constructed ("trained") through the data-set generated by the sampling of the physical model. Once trained, they are going to be used in place of the original physical model.

As it can be seen, the ROMs will be constructed considering four features (sigma-A, sigma$B$, decay $-A$, and decay $-B$ ) and two targets ( $A$ and $B)$.

\section{Distributions:}

raven/tests/framework/user_guide/ReducedOrderModeling/reducedorderModeling.xml

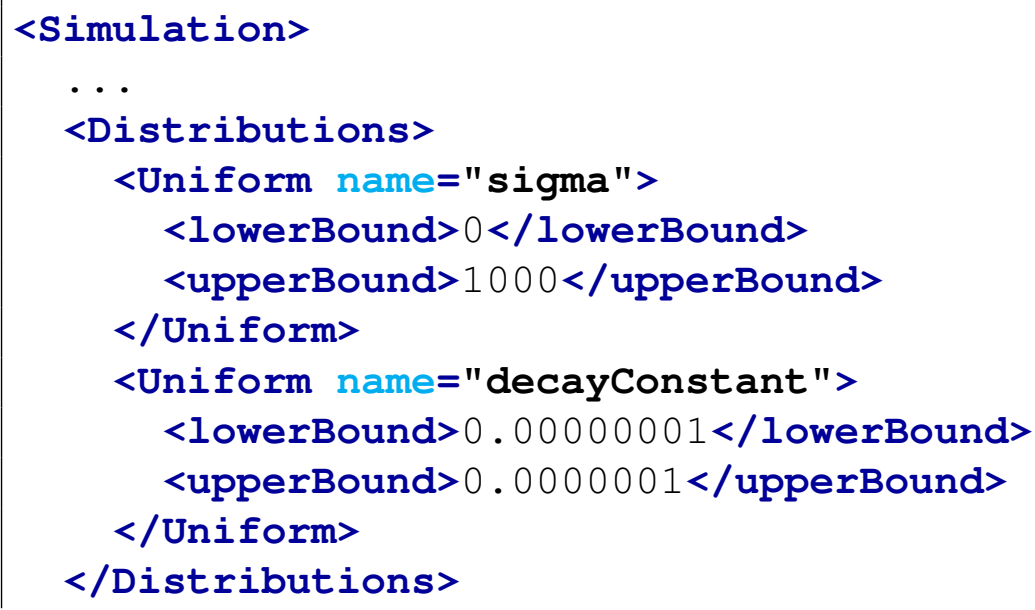


...

$</$ Simulation $>$

In the Distributions XML section, the stochastic model for the uncertainties are reported. In this case two distributions are defined:

- sigma $\sim \mathbb{U}(0,1000)$, used to model the uncertainties associated with the Model sigma$A$ and sigma-B;

- decayConstant $\sim \mathbb{U}(1 e-8,1 e-7)$, used to model the uncertainties associated with the Model decay- $A$ and decay- $B$.

\section{Samplers:}

raven/tests/framework/user_guide/ReducedorderModeling/reducedorderModeling.xml

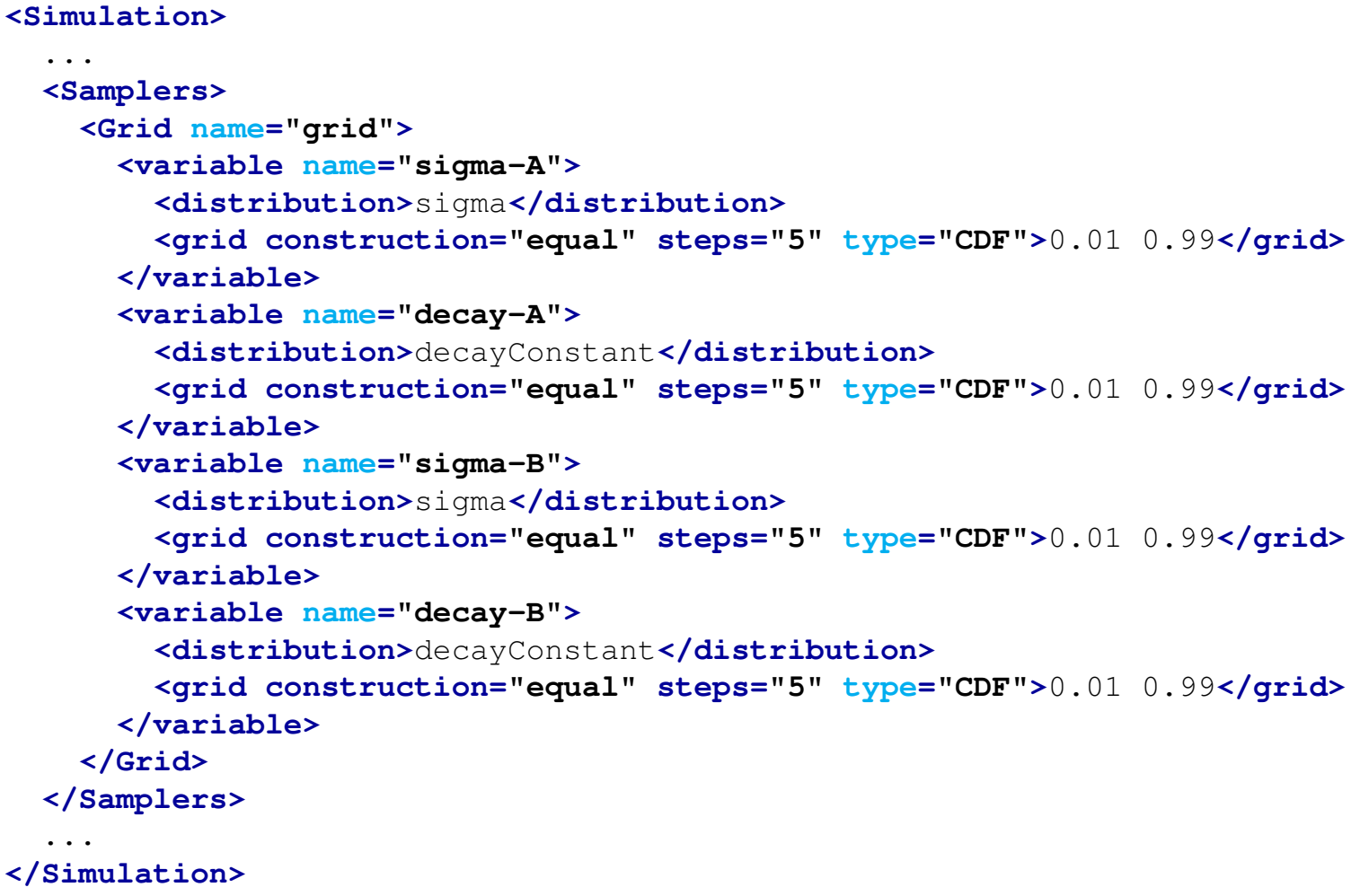

To obtain the data-set through which the ROMs are going to be constructed, a Grid sampling approach is here employed.

\section{DataObjects:}

raven/tests/framework/user_guide/ReducedorderModeling/reducedorderModeling.xml

$<$ Simulation $>$

...

<DataObjects $>$ 


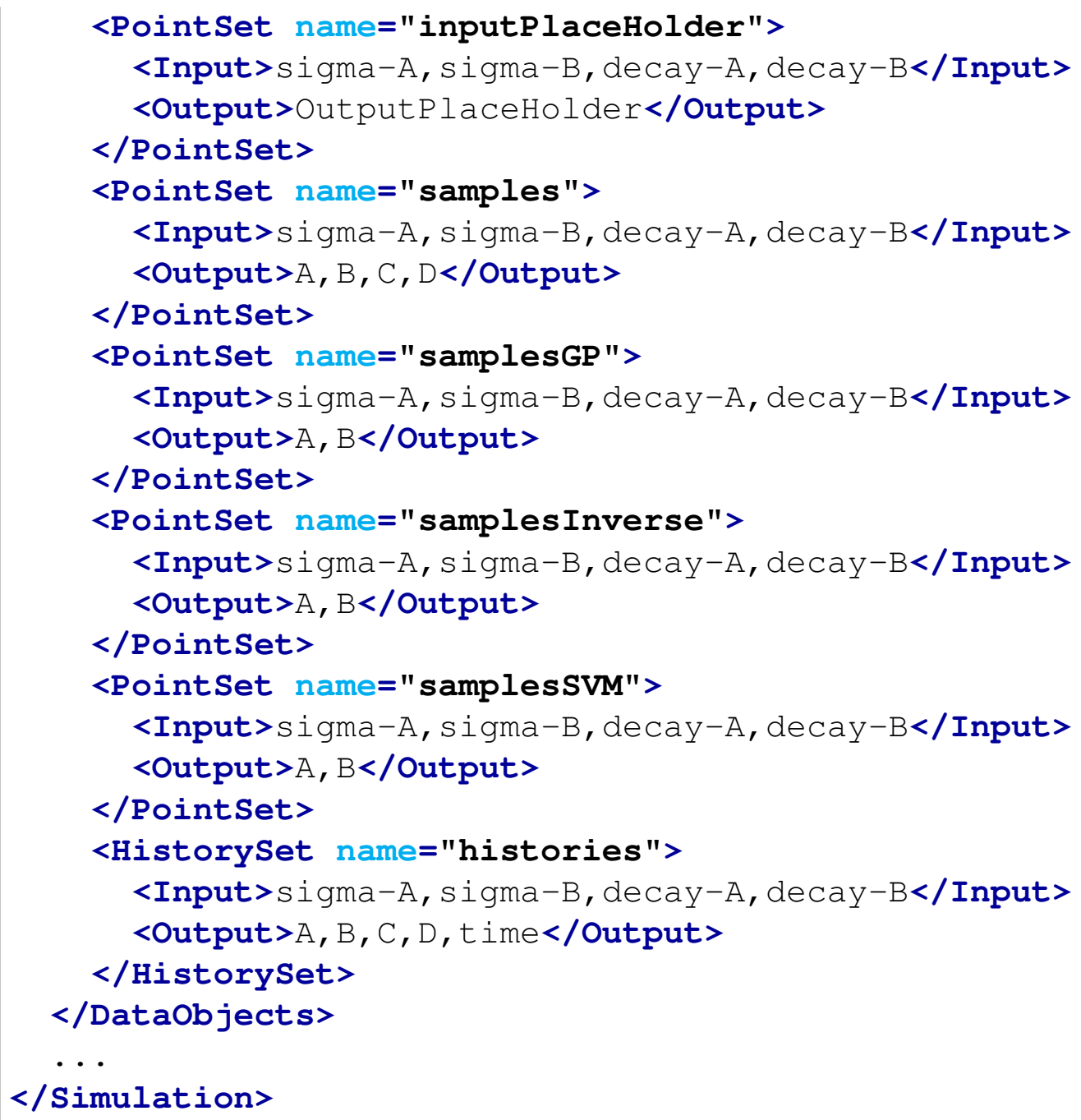

Int this block, six DataObjects are defined: 1) PointSet named "samples" used to collect the final outcomes of the code, 2) HistorySet named "histories" in which the full time responses of the variables $A, B, C, D$ are going to be stored, 3) PointSet named "inputPlaceHolder" used in the role of <Input> for the ROMs sampling; 4) PointSet named "samplesGP" used to collect the final outcomes (sampling) of the GP ROM; 5) PointSet named "samplesInverse" used to collect the final outcomes (sampling) of the IDW ROM; 6) PointSet named "samplesSVM" used to collect the final outcomes (sampling) of the SVM ROM.

\section{OutStreams:}



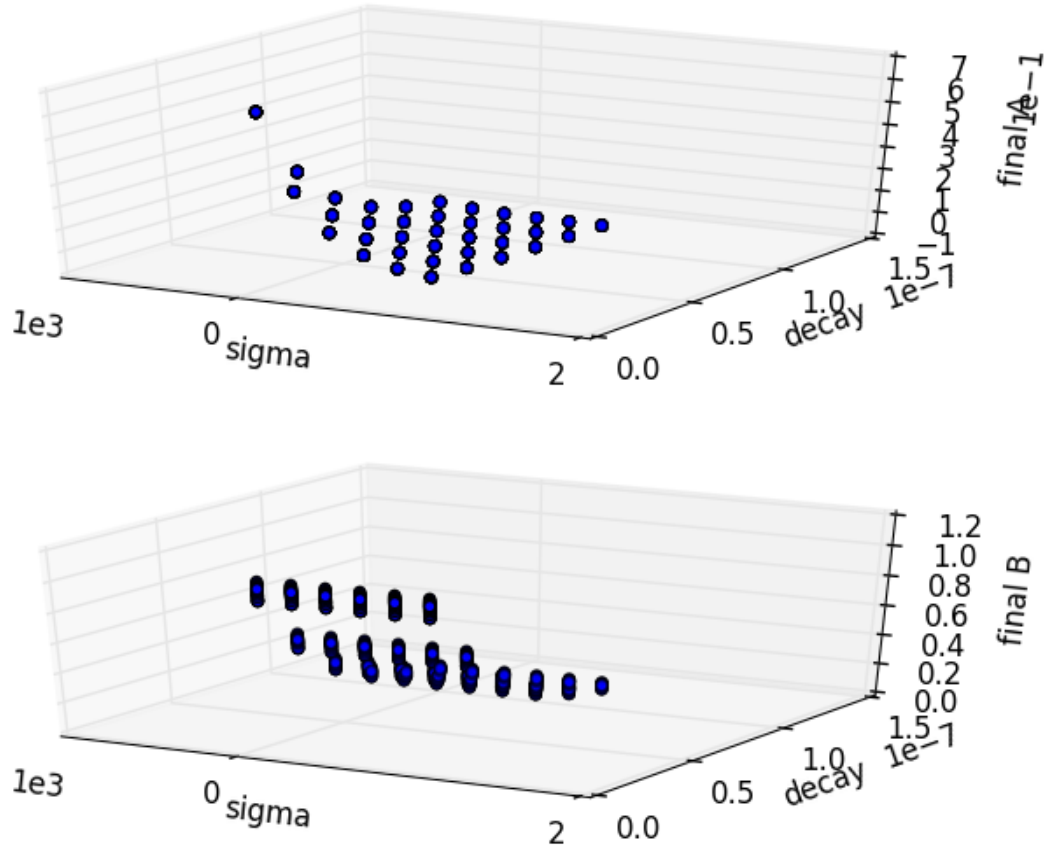

Figure 26. Plot of the samples generated by the Grid sampling for variables $A, B$.

raven/tests/framework/user_guide/ReducedorderModeling/reducedOrderModeling.xml

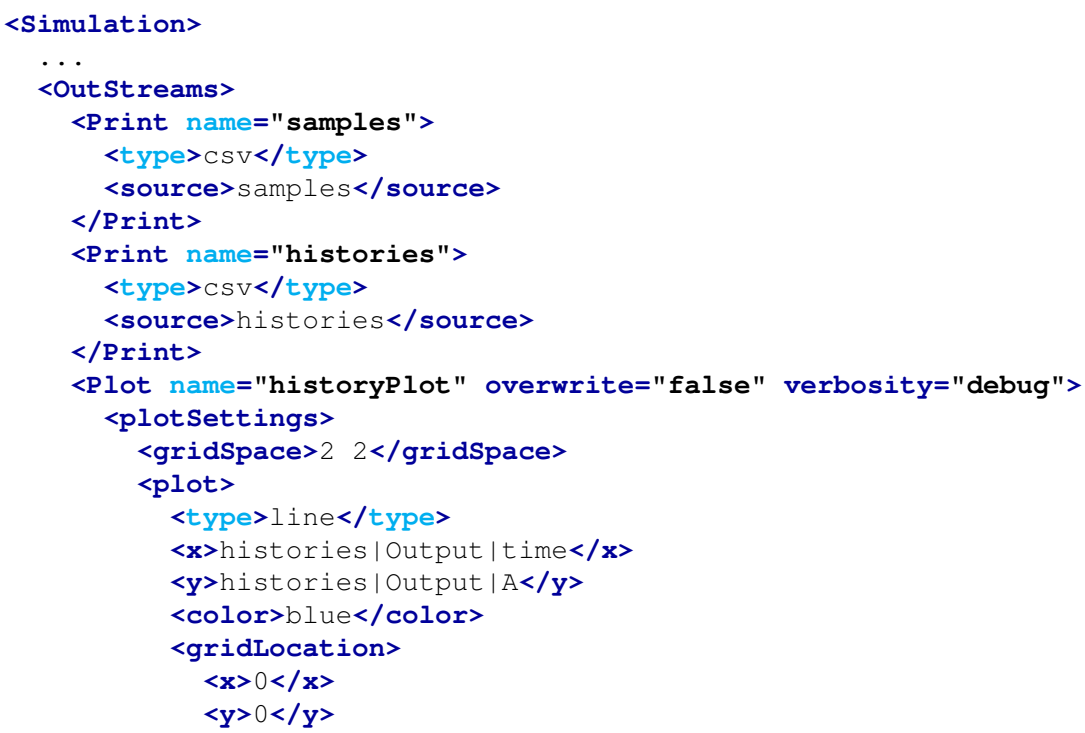




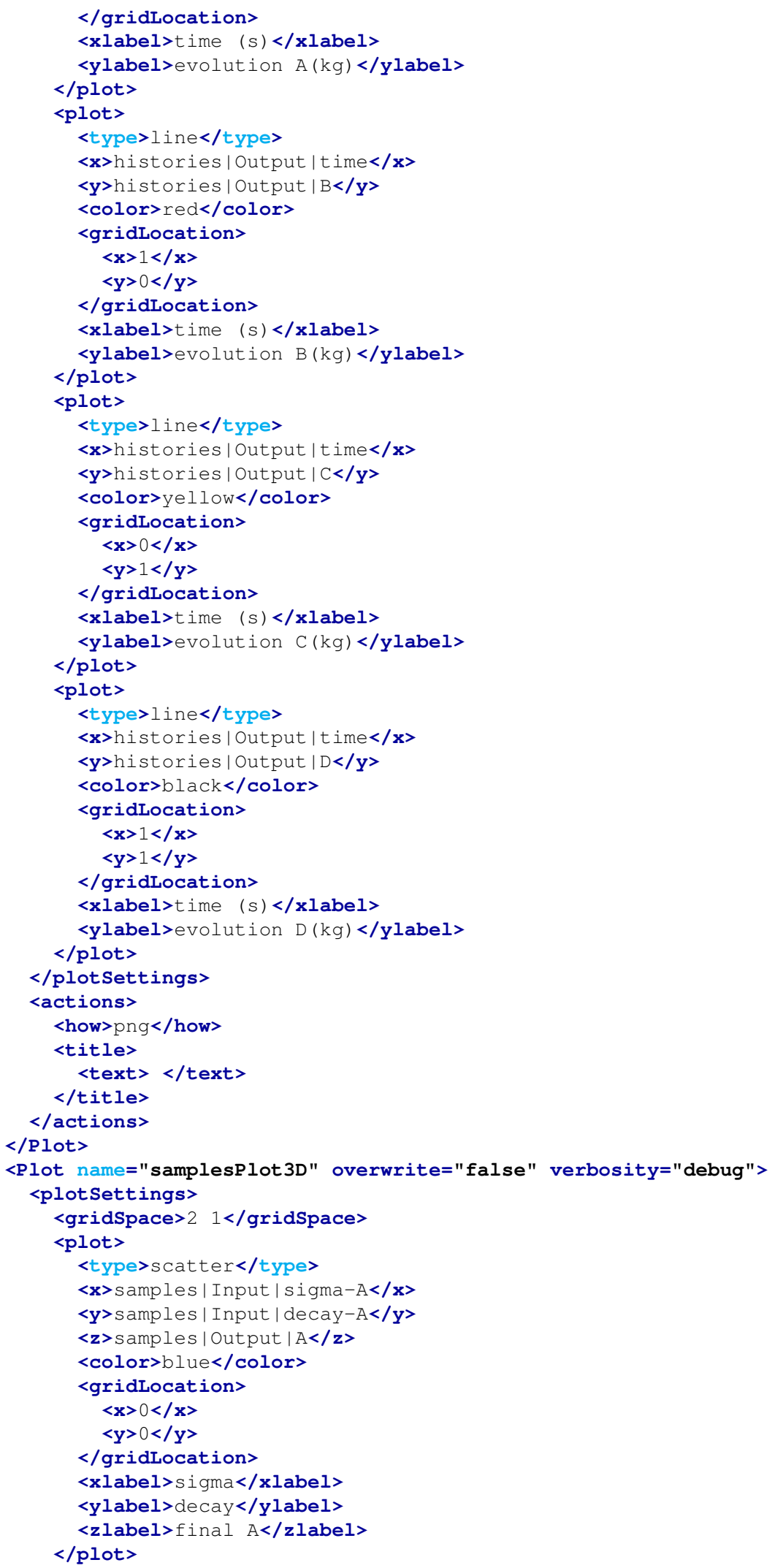




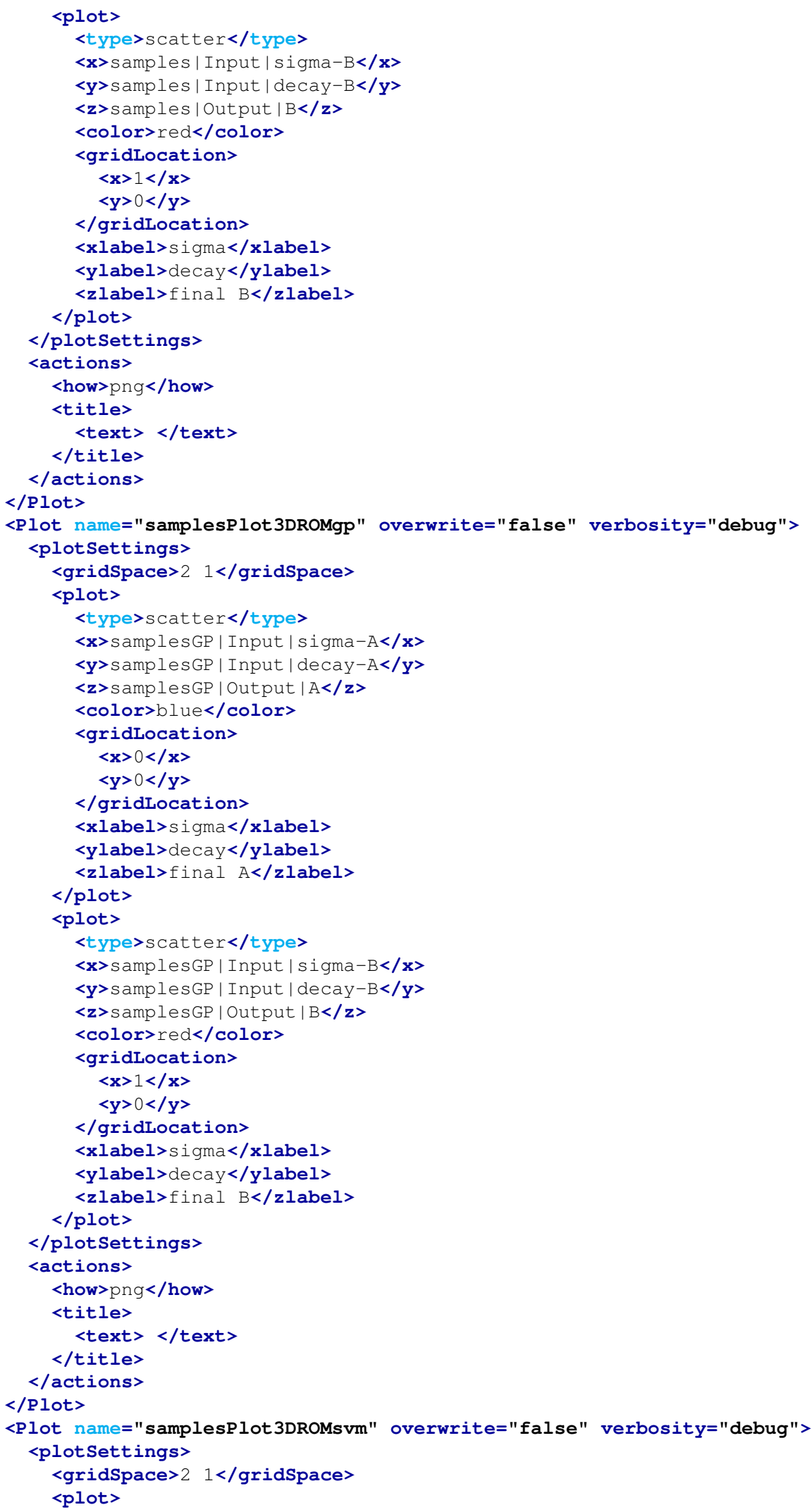




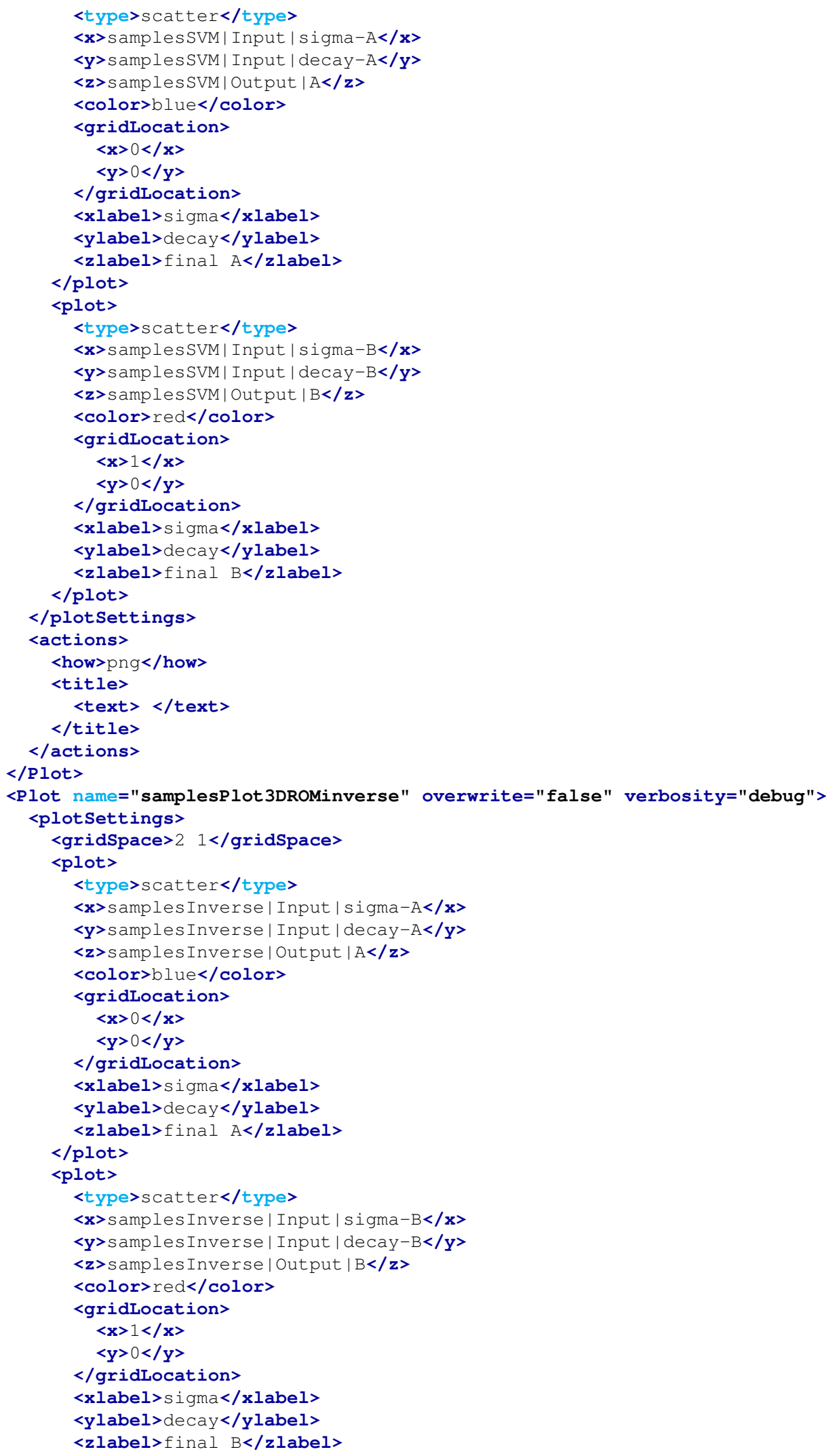




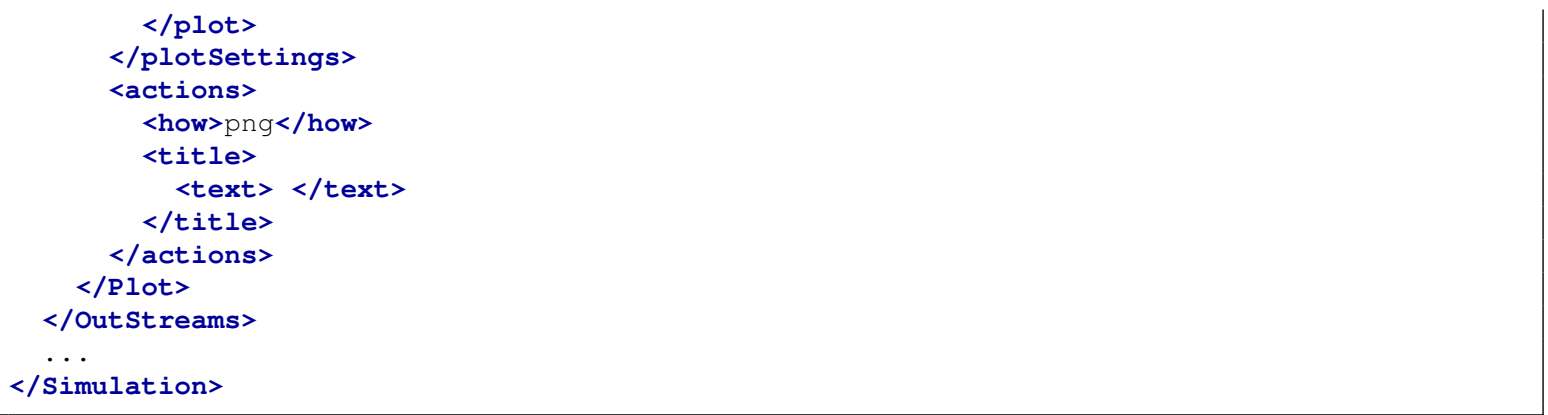

This model makes use of two Print OutStreams and five Plot OutStreams:

- "samples," which writes the contents of the point-wise training samples to CSV,

- "histories," which writes the contents of the history-wise training samples to linked CSVs,

- "historyPlot," which plots the evolution of the training samples,

- "samplesPlot3D," which plots the final state of the training samples with relation to the outputs of interest,

- "samplesPlot3DROMgp," which plots the validation samples of the Gaussian Process ROM,

- "samplesPlot3DROMsvm," which plots the validation samples of the Support-Vector Machine ROM,

- "samplesPlot3Dinverse," which plots the validation samples of the multidimensional Inverse Weight ROM.

The 3D plots of the samples as well as the ROM samples can be used as a view-norm validation of the ROMs.

\section{Steps:}

raven/tests/framework/user_guide/ReducedOrderModeling/reducedorderModeling.xml

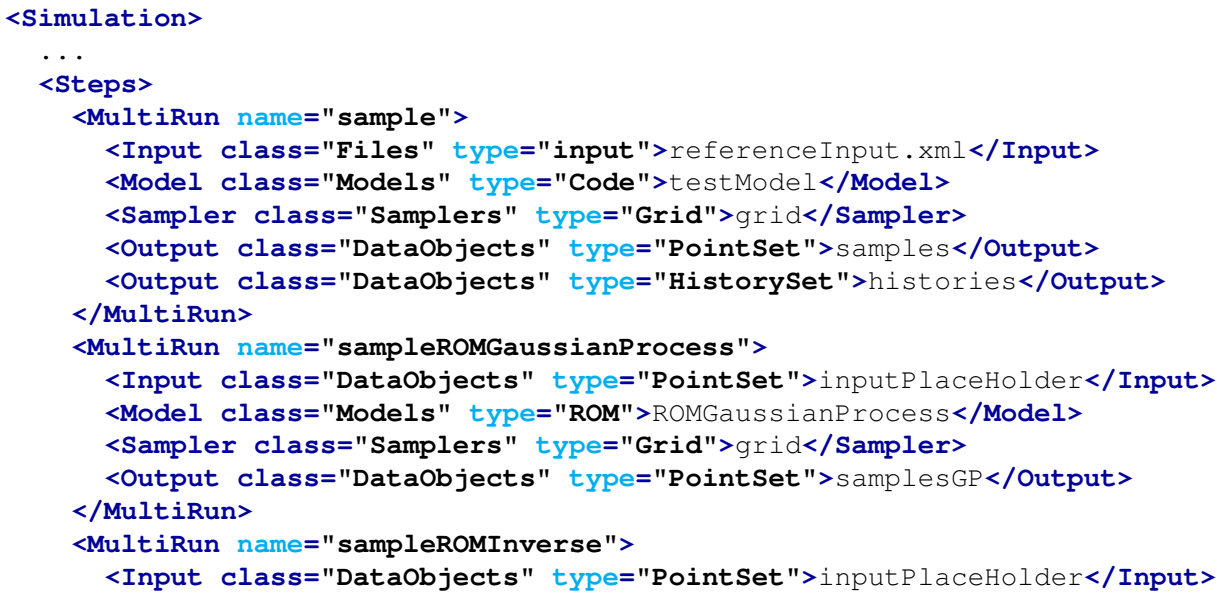




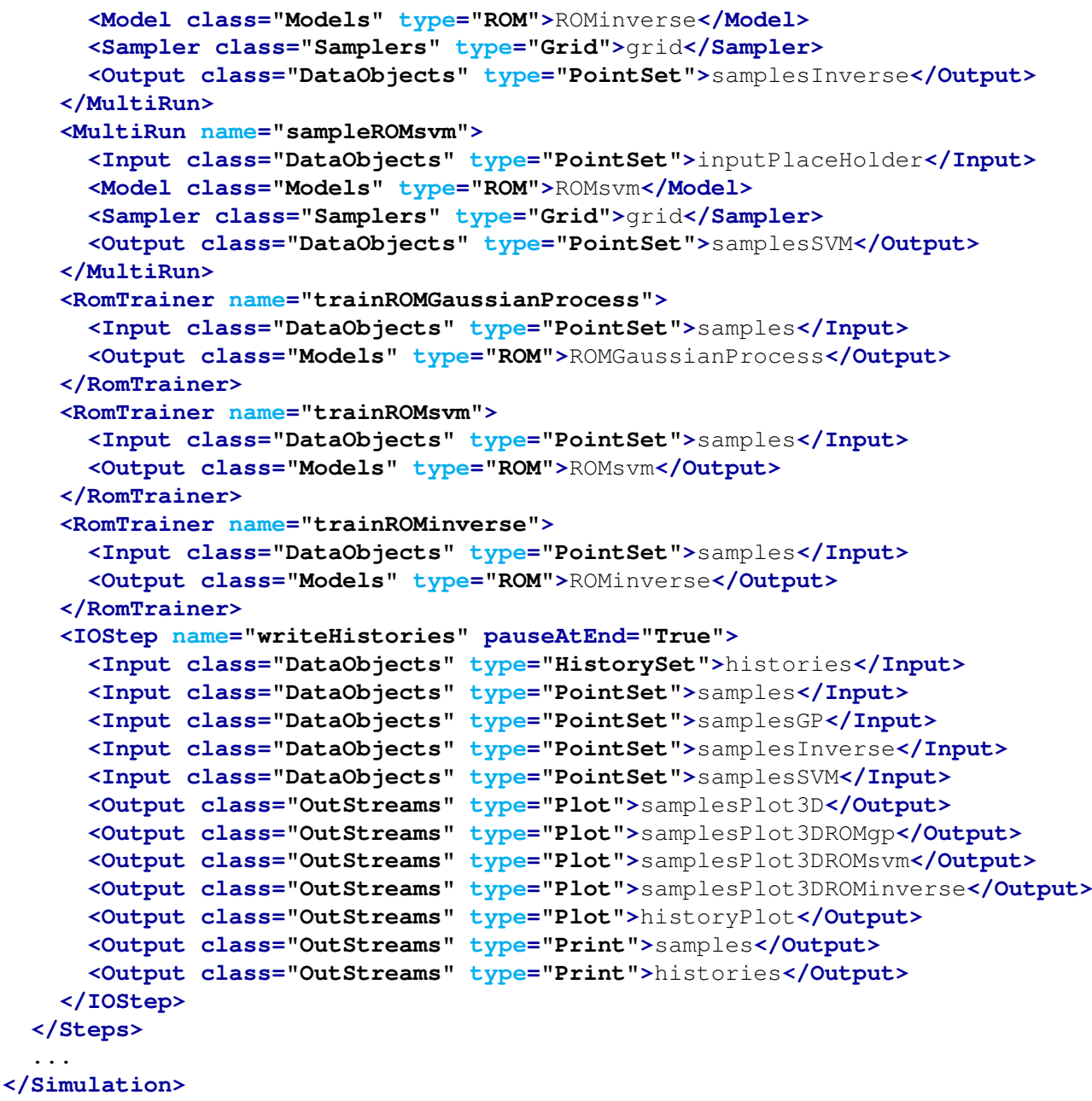

Finally, all the previously defined Entities can be combined in the <Steps> block. As inferable, eight <steps $>$ have been inputted:

- <MultiRun> named "sample", used to run the multiple instances of the driven code and collect the outputs in the two DataObjects. As it can be seen, the <Sampler $>$ is inputted to communicate to the Step that the driven code needs to be perturbed through the Grid sampling strategy;

- <RomTrainer> named "trainROMGaussianProcess", used to construct ("train") the GP ROM, based on the data-set generated in the "sample" Step;

- <RomTrainer> named "trainROMsvm", used to construct ("train") the SVM ROM, based on the data-set generated in the "sample" Step;

- <RomTrainer> named "trainROMinverse", used to construct ("train") the IDW ROM, based on the data-set generated in the "sample" Step;

- <MultiRun> named "sampleROMGaussianProcess", used to run the multiple instances of the previously constructed GP ROM and collect the outputs in the PointSet 

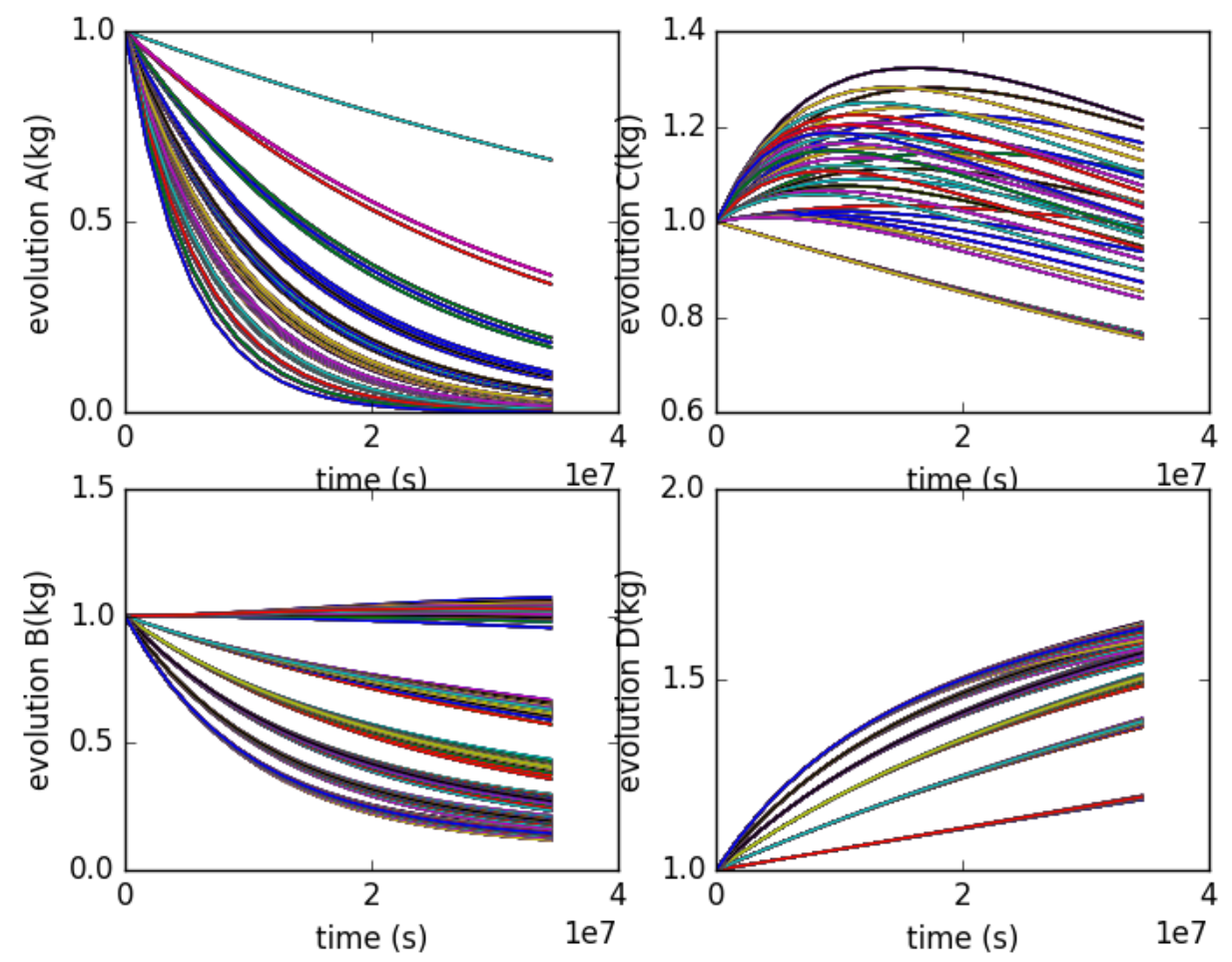

Figure 27. Plot of the histories generated by the Grid method for variables $A, B, C, D$.

DataObject. As it can be seen, the same < Sampler > used for perturbing the original model is here used.

- <MultiRun> named "sampleROMsvm", used to run the multiple instances of the previously constructed Support Vector Machine ROM and collect the outputs in the PointSet DataObject. As it can be seen, the same <Sampler> used for perturbing the original model is here used.

- $<$ MultiRun> named "sampleROMInverse", used to run the multiple instances of the previously constructed Inverse Distance Weight ROM and collect the outputs in the PointSet DataObject. As it can be seen, the same <Sampler> used for perturbing the original model is here used.

- <IOStep> named "writeHistories", used to 1) export the "histories" and "samples" DataObjects Entity in a CSV file and 2) plot the responses of the sampling performed on the physical model, GP ROM, SVM ROM and IDW ROM in PNG files and on the screen. 

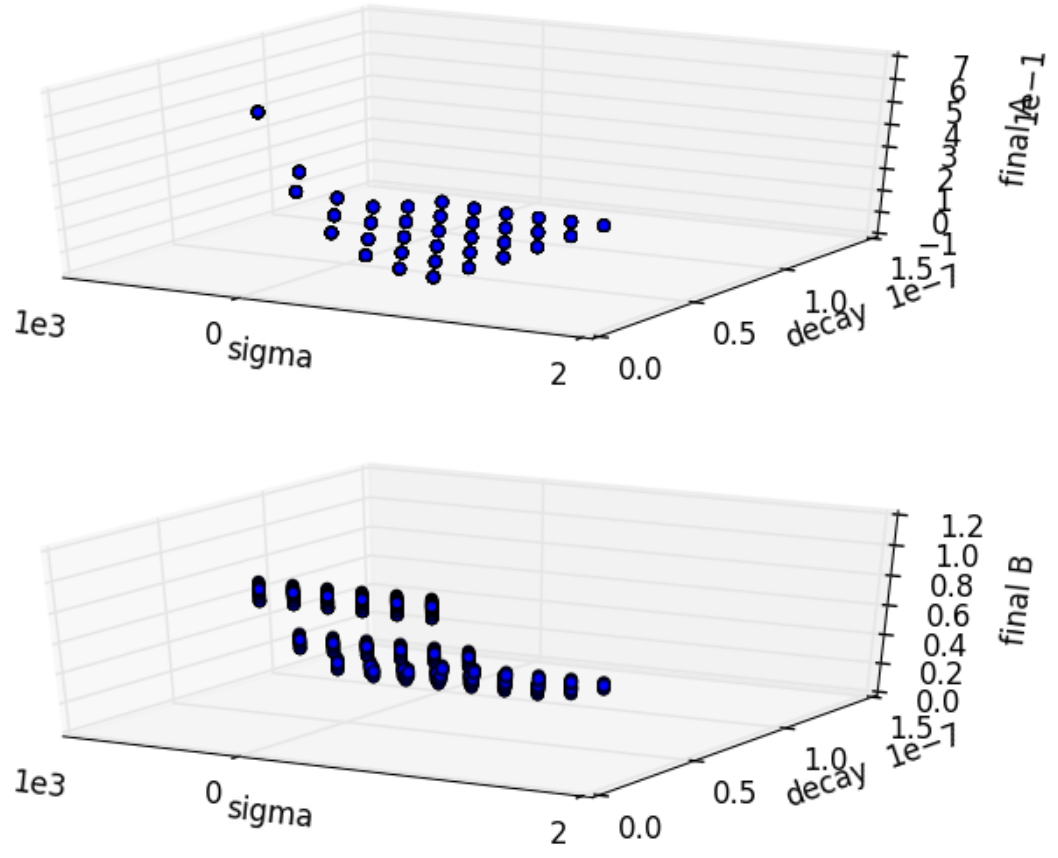

Figure 28. Plot of the samples generated by the Grid sampling applied on the Gaussian Process ROM for variables $A, B$

Figure 27 shows the evolution of the outputs $A, B, C, D$ under uncertainties. Figure 26 shows the final responses of $A a n d B$ of the sampling employed using the driven code.

Figures 28, 29 and 30 show the final responses of $A a n d B$ of the sampling employed using the Gaussian Process, Support Vector Machines and Inverse Distance Weight ROMs, respectively. It can be clearly noticed that the responses of the ROMs perfectly match the outcomes coming from the original model (see Figure 26). 

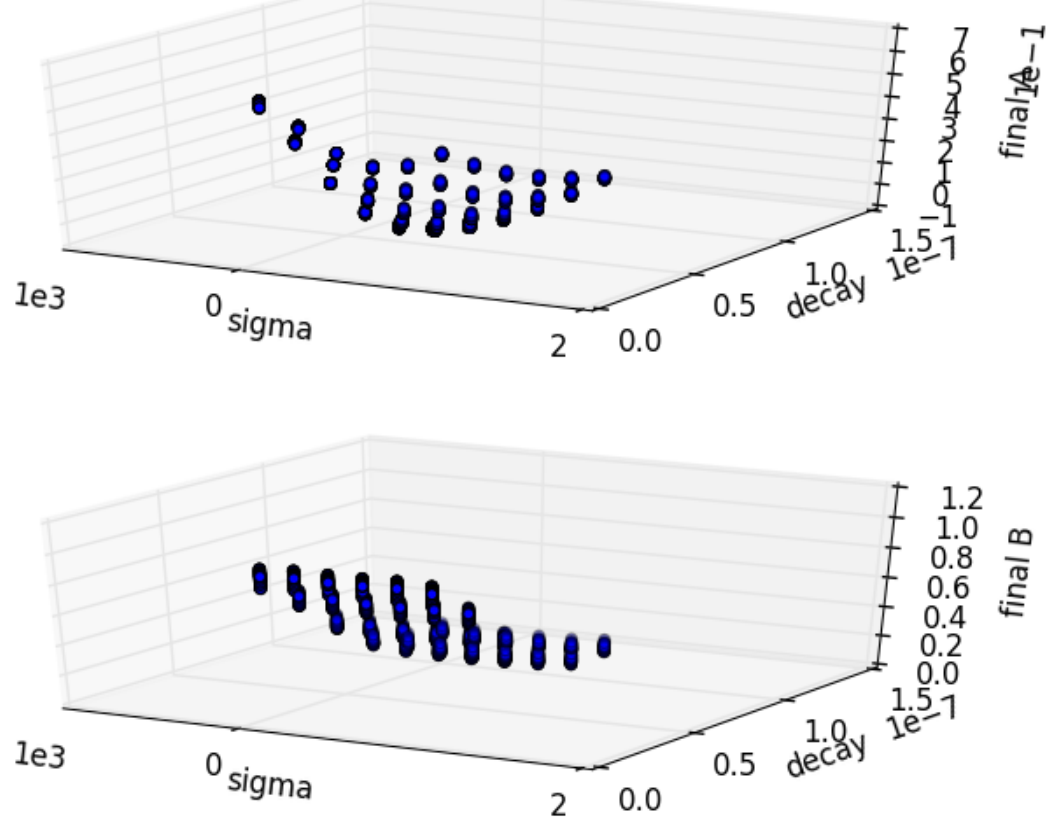

Figure 29. Plot of the samples generated by the Grid sampling applied on the Support Vector Machine ROM for variables $A, B$ 

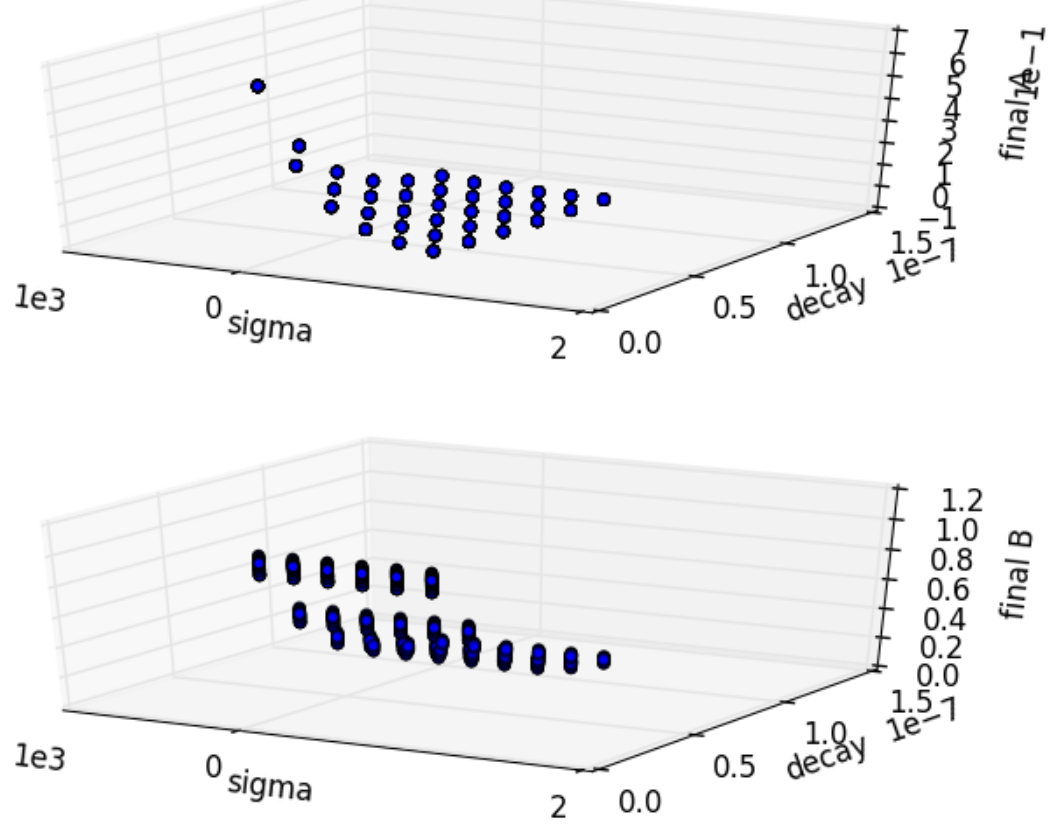

Figure 30. Plot of the samples generated by the Grid sampling applied on the Inverse Distance Weight ROM for variables $A, B$ 


\section{Statistical Analysis through RAVEN}

In order to perform a complete analysis of a system under uncertainties, it is crucial to be able to compute all the statistical moments of one or even multiple FOMs. In addition, it is essential to identify the correlation among different FOMs toward a specific input space.

RAVEN is able to compute the most important statistical moments: such as:

\section{Expected Value}

2. Standard Deviation

3. Variance

4. variationCoefficient

5. Skewness

6. Kurtosis

7. Median

8. Percentile.

In addition, RAVEN fully supports the computation of all of the statistical moments defined to "measure" the correlation among variables/parameters/FOMs:

\section{Covariance matrix}

2. Normalized Sensitivity matrix

3. Variance Dependent Sensitivity matrix

4. Sensitivity matrix

5. Pearson matrix.

The goals of this section is to show how to:

1. Set up a sampling strategy to perform a final statistical analysis perturbing a driven code

2. Compute all the statistical moments and correlation/covariance metrics. 
In order to accomplish these tasks, the following RAVEN Entities (XML blocks in the input files) need to be defined:

\section{RunInfo:}

raven/tests/framework/user_guide/StatisticalAnalysis/statisticalAnalysis.xml

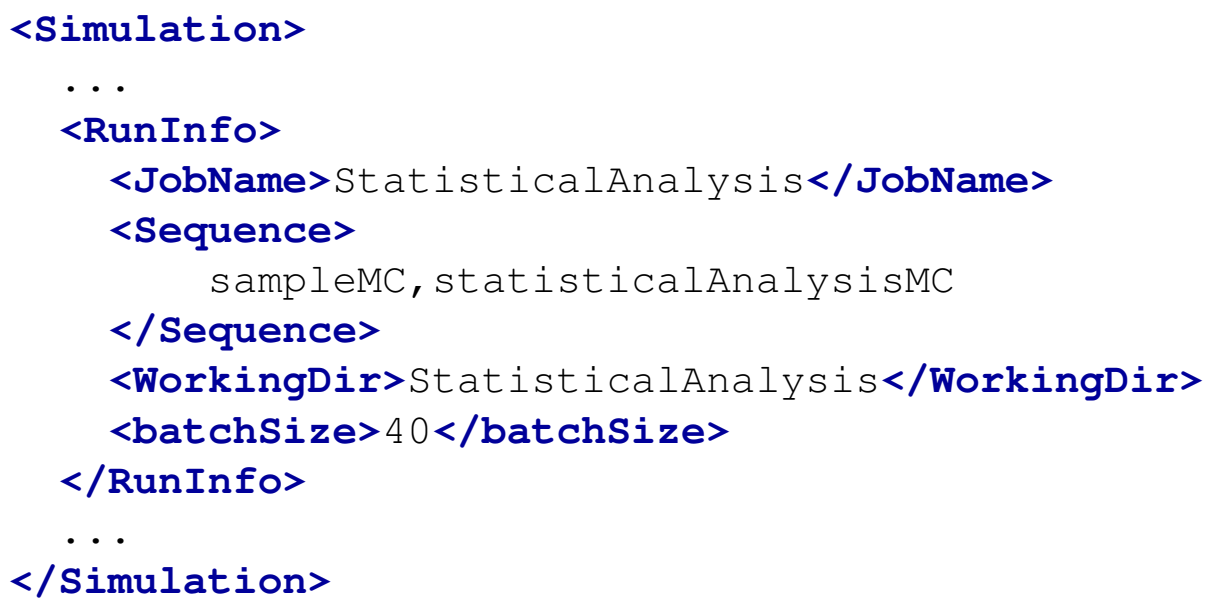

As shown in the other examples, the RunInfo Entity is intended to set up the desired analysis . In this specific case, two steps (<Sequence>) are sequentially run using forty processors (<batchSize>).

In the first step, the original physical model is sampled. The obtained results are analyzed with the Statistical Post-Processor.

\section{Files:}

raven/tests/framework/user_guide/StatisticalAnalysis/statisticalAnalysis.xml

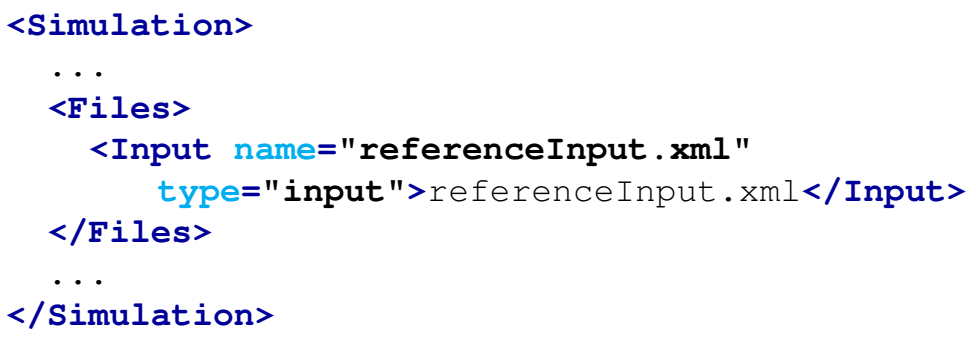

Since the driven code uses a single input file, in this section the original input is placed. As detailed in the user manual the attribute name represents the alias that is going to be used in all the other input blocks in order to refer to this file.

In addition, the output file of the PostProcess Step is here defined (XML format).

\section{Models:}

raven/tests/framework/user_guide/StatisticalAnalysis/statisticalAnalysis.xml 


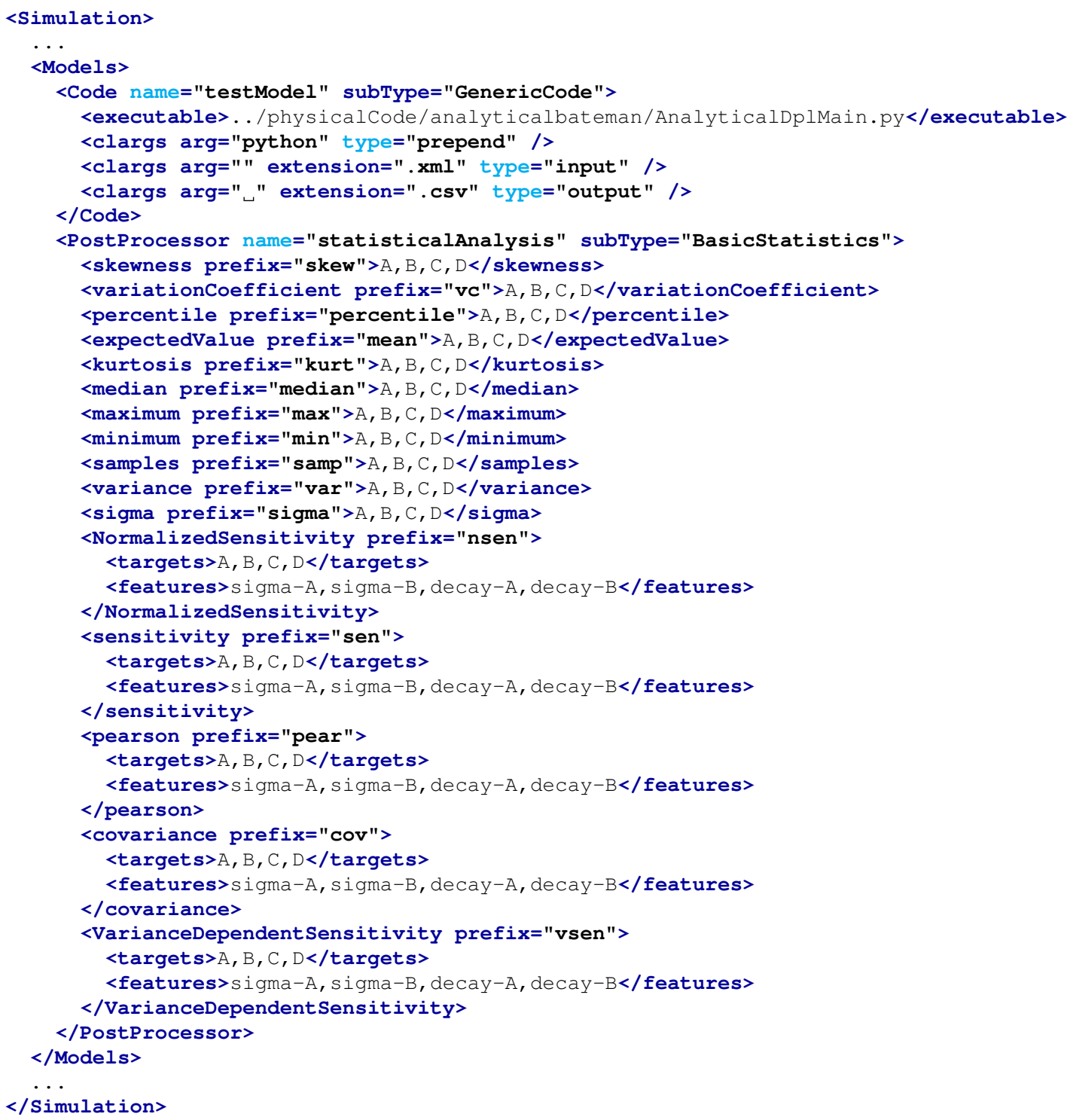

The goal of this example is to show how the principal statistical FOMs can be computed through RAVEN.

Indeed, in addition to the previously explained Code model, a Post-Processor model (BasicStatistics) is here specified. Note that the post-process step is performed on all the variables with respect to the parameters used in this example ( $A, B, C$ and $D$ with respect to sigma - A, sigma $-B$, decay $-A$, and decay $-B$ ).

\section{Distributions:}

raven/tests/framework/user_guide/StatisticalAnalysis/statisticalAnalysis.xml

\section{$<$ Simulation $>$}




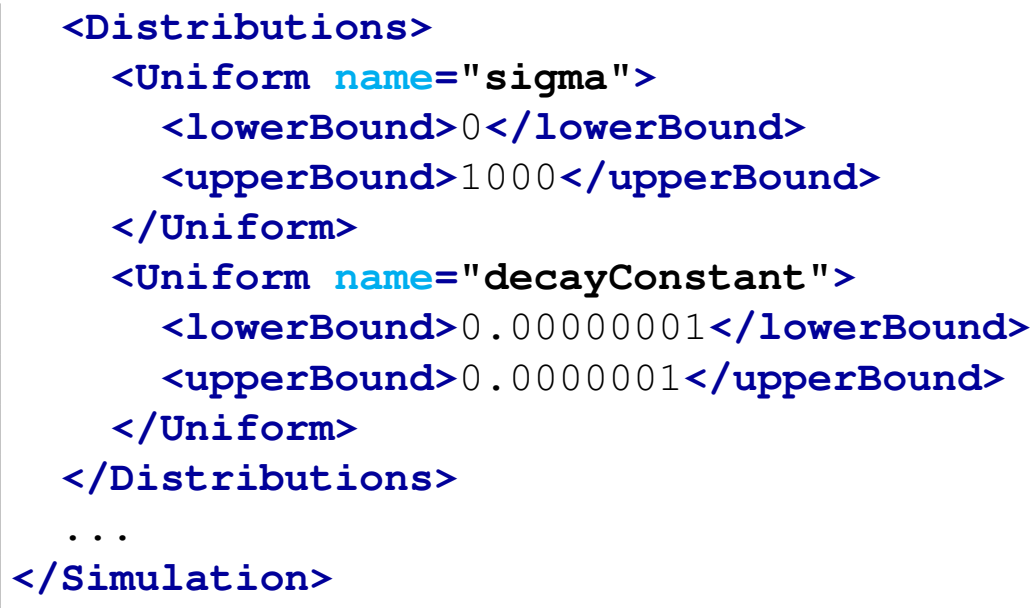

$</$ Simulation $>$

In the Distributions XML section, the stochastic models for the uncertainties are reported. In this case 2 distributions are defined:

- sigma $\sim \mathbb{U}(0,1000)$, used to model the uncertainties associated with the Model sigma$A$ and sigma-B

- decayConstant $\sim \mathbb{U}(1 e-8,1 e-7)$, used to model the uncertainties associated with the Model decay-A and decay-B.

\section{Samplers:}

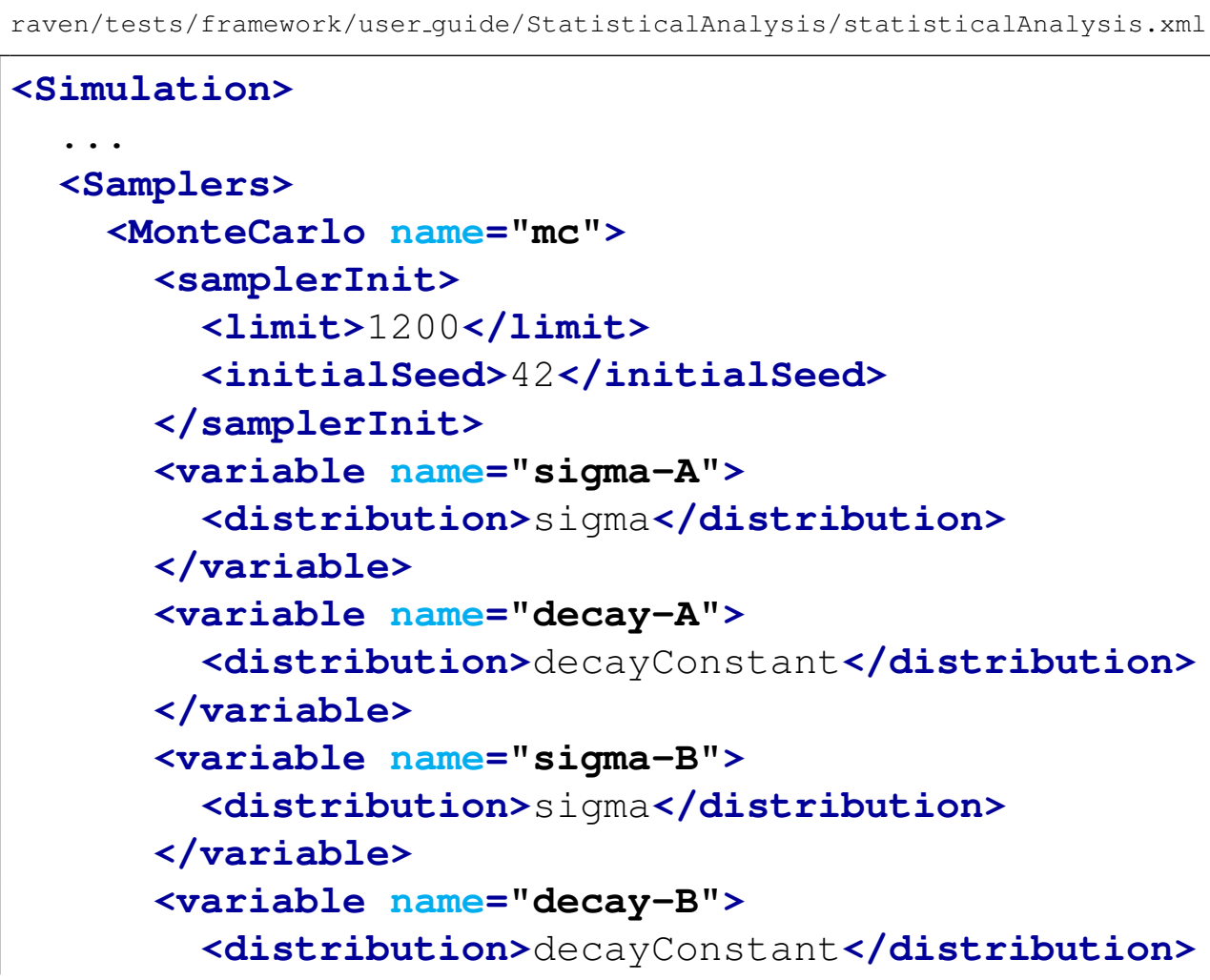




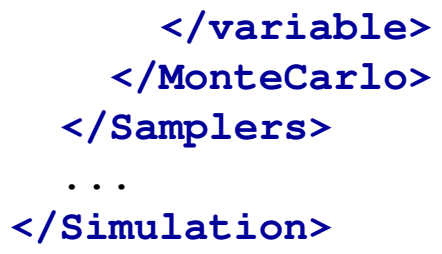

In order to obtained the data-set through which the statistical FOMs need to be computed, a MonteCarlo sampling approach is here employed.

\section{DataObjects:}

raven/tests/framework/user_guide/StatisticalAnalysis/statisticalAnalysis.xml

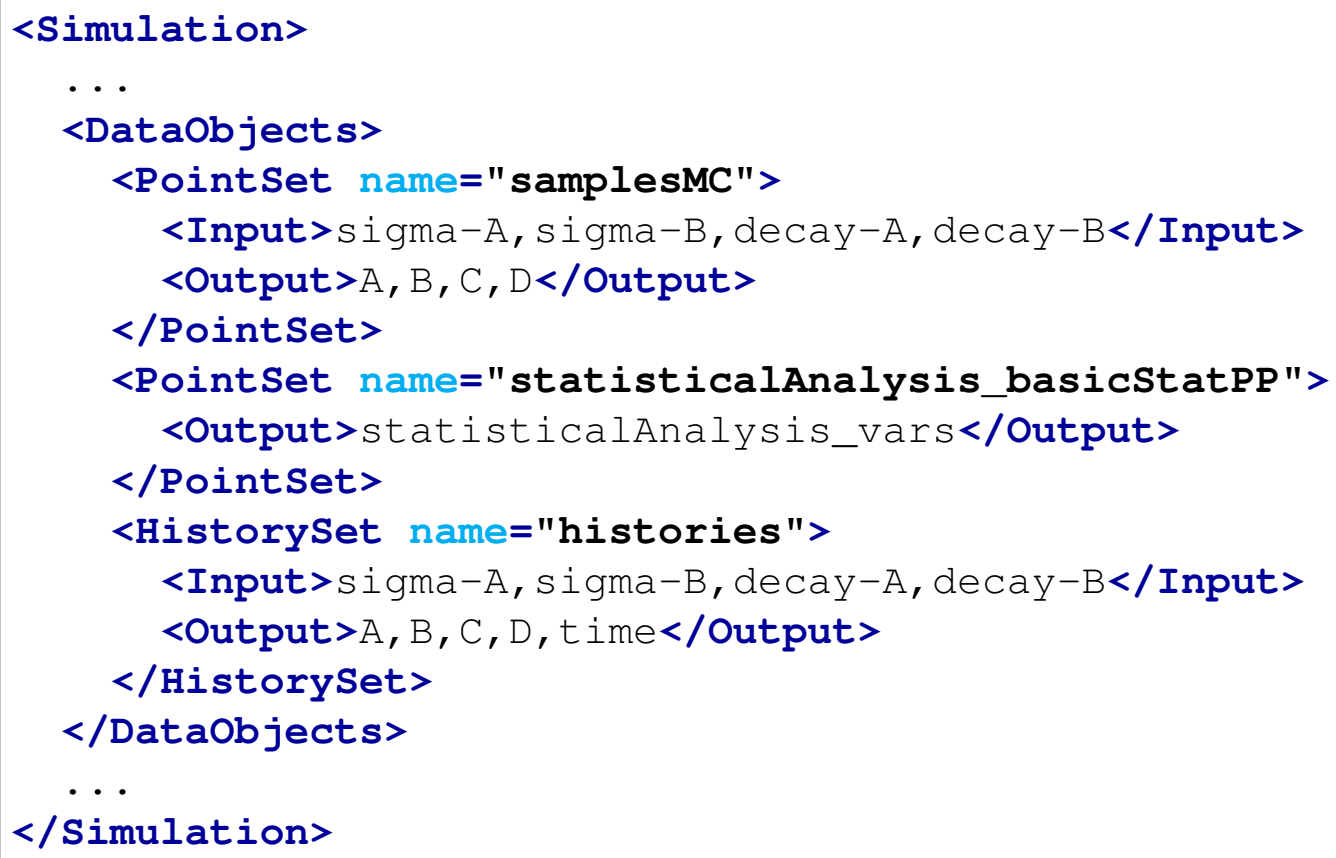

Int this block, two DataObjects are defined: 1) PointSet named "samplesMC" used to collect the final outcomes of the code, 2) HistorySet named "histories" in which the full time responses of the variables $A, B, C, D$ are going to be stored.

\section{Steps:}

raven/tests/framework/user_guide/StatisticalAnalysis/statisticalAnalysis.xml

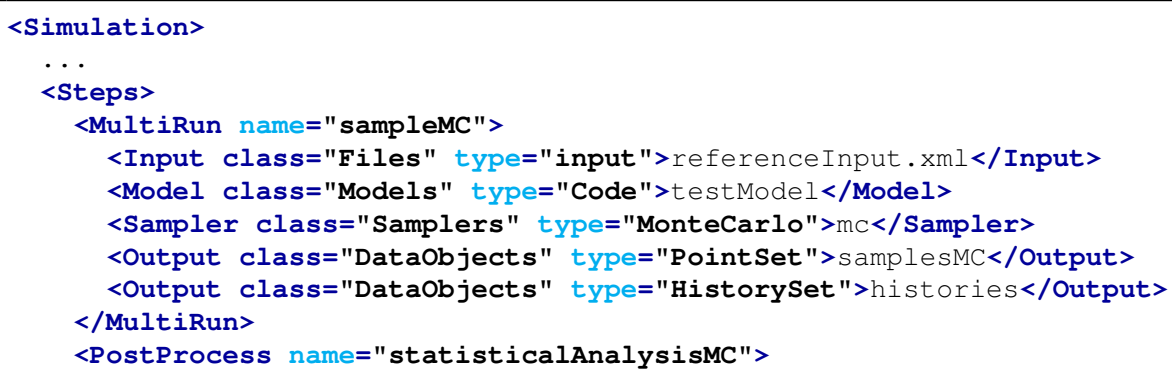




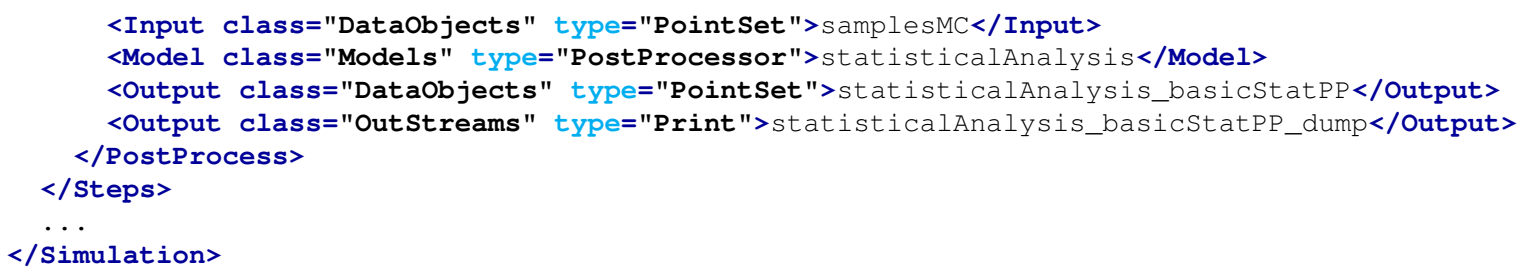

Finally, all the previously defined Entities can be combined in the <Steps > block. As inferable, 2 <steps > have been inputted:

- <MultiRun> named "sampleMC", used to run the multiple instances of the driven code and collect the outputs in the two DataObjects. As it can be seen, the <Sampler> is inputted to communicate to the Step that the driven code needs to be perturbed through the Grid sampling strategy.

- <PostProcess> named "statisticalAnalysisMC", used compute all the statistical moments and FOMs based on the data obtained through the sampling strategy. As it can be noticed, the <Output> of the "sampleMC" Step is the <Input> of the "statisticalAnalysisMC" Step.

Tables 2-6 show all the results of the PostProcess step. 


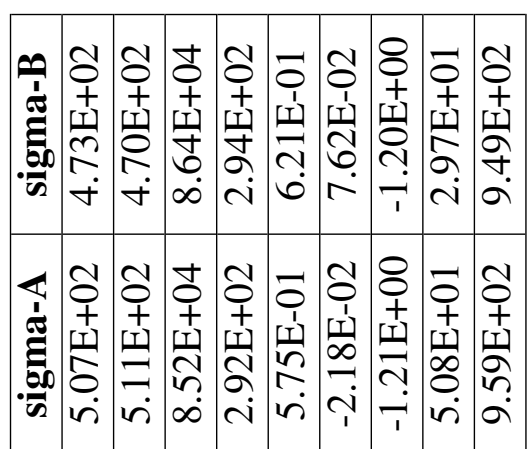

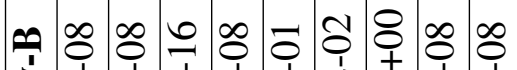

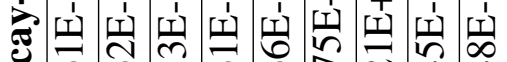

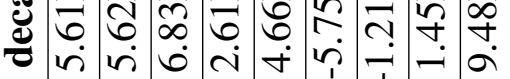

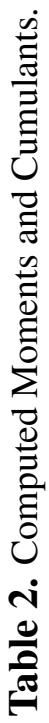

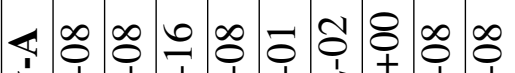

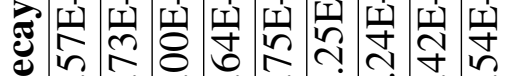
-

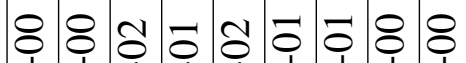

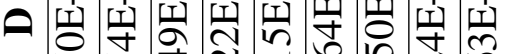
ڤִ

-

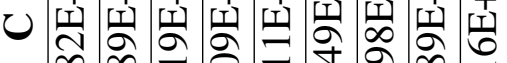
$\infty \infty=0 .+0$

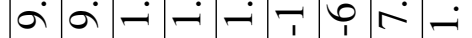

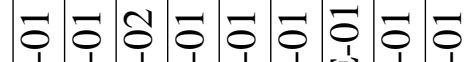

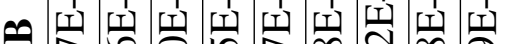
ลิ

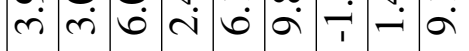

ปิธิ์

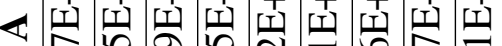

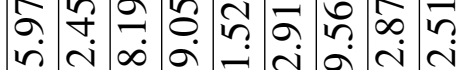

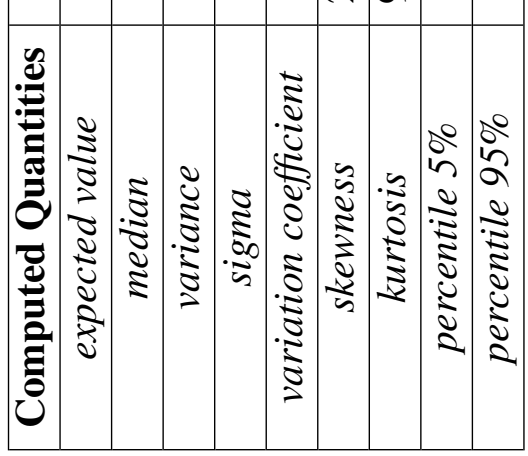

^

ซ

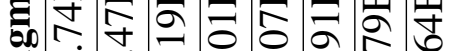

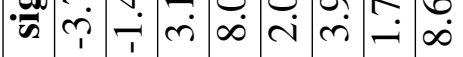

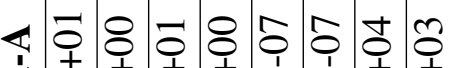

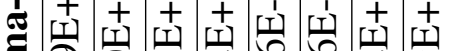

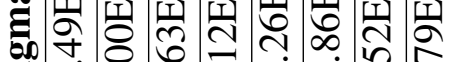

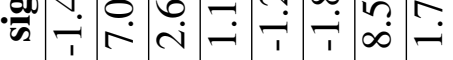

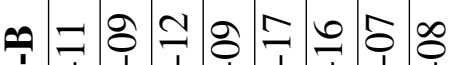
خ' 7 '1 1 '1 త্ঠ் 守合

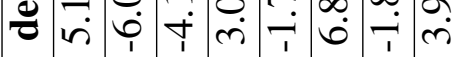

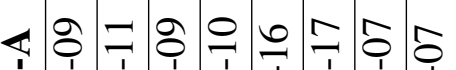

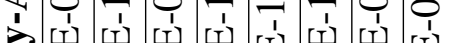

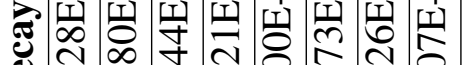
ฮِّ ப인

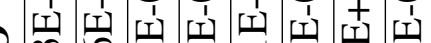

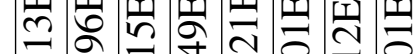

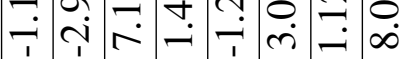

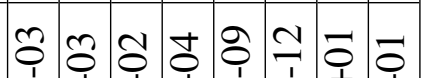

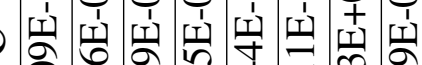

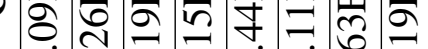
$\dot{i} \sim \vec{r}+\dot{i} \dot{r}$

అิْ

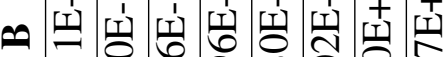
= 8 ○े $\begin{array}{lllllll}1 & 0 & 4 & 1 & 1 & 1\end{array}$ అ̂́

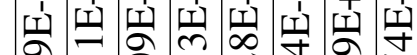
Ә $\infty \rightarrow \dot{r} \rightarrow \dot{r} \rightarrow \vec{r}$

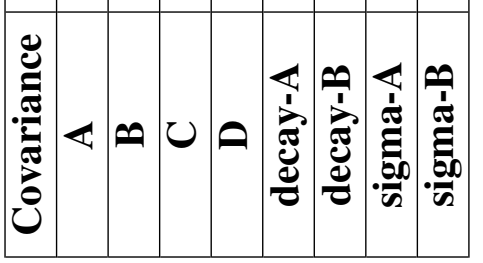




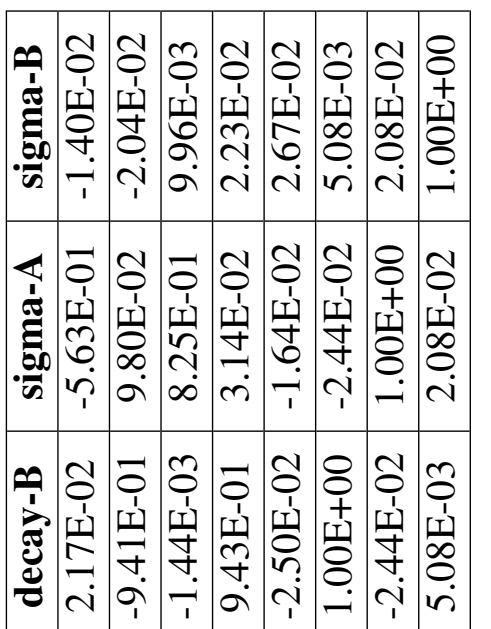

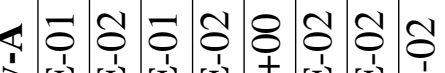

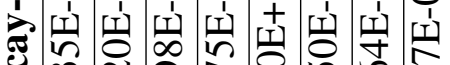

త্ঠ

-

ઈิ

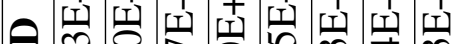

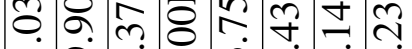

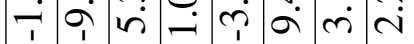

పิ

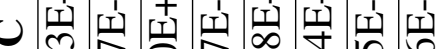

๓

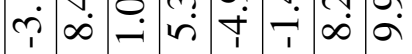

ত̂

^ II I I I

ปิ

i $\infty \sigma_{1}-a_{1} a_{1}$

ᄋ

4 II Iس

I

穴

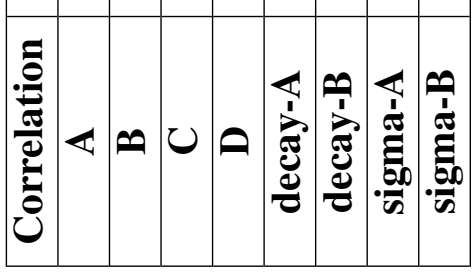

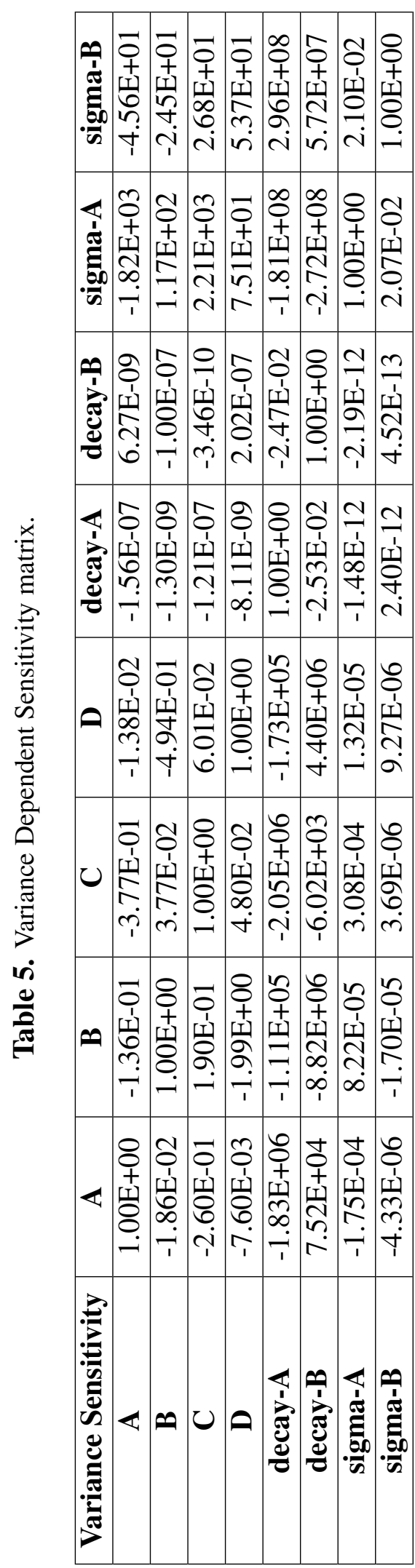




\section{Data Mining through RAVEN}

Data mining is the computational process of discovering patterns in large data sets ("big data") involving methods at the intersection of artificial intelligence, machine learning, statistics, and database systems. The overall goal of the data mining process is to extract information from a data set and transform it into an understandable structure for further use.

RAVEN has support of several different data mining algorithms, such as:

\section{Hierarchical methodologies}

2. K-Means

3. Mean-Shift, etc.

The goals of this section are about learning how to:

1. Set up a sampling strategy to apply clustering algorithms, perturbing a driven code

2. Analyze the data using clustering algorithms.

To accomplish these tasks, the following RAVEN Entities (XML blocks in the input files) need to be defined:

\section{RunInfo:}

raven/tests/framework/user_guide/DataMining/dataMiningAnalysis.xml

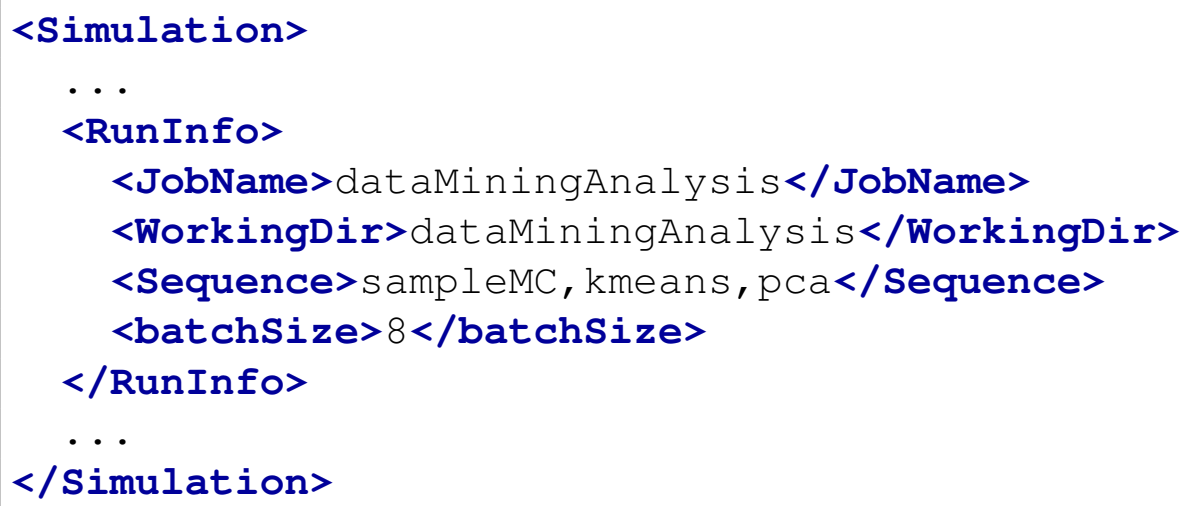

The RunInfo Entity is intended to set up the analysis sequence that needs to be performed. In this specific case, two steps (<Sequence $>$ ) are sequentially run using forty processors (<batchSize>).

In the first step, the original physical model is going to be sampled. The obtained results are going to be analyzed with data mining algorithms. 


\section{Files:}

raven/tests/framework/user_guide/DataMining/dataMiningAnalysis.xml

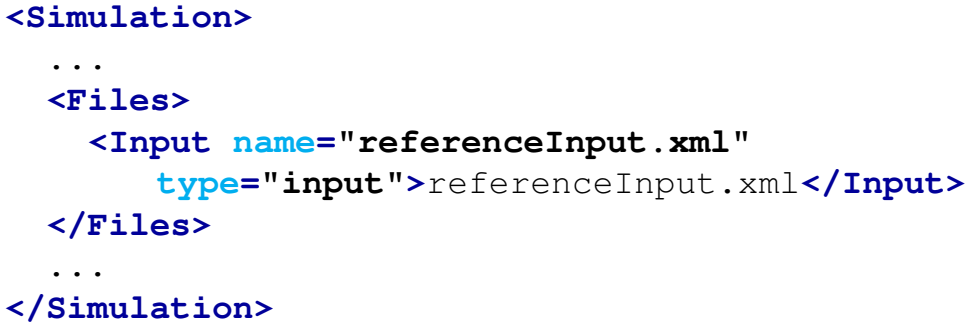

Since the driven code uses a single input file, in this section the original input is placed. The attribute name represents the alias that is going to be used in all the other input blocks in order to refer to this file.

\section{Models:}

raven/tests/framework/user_guide/DataMining/dataMiningAnalysis.xml

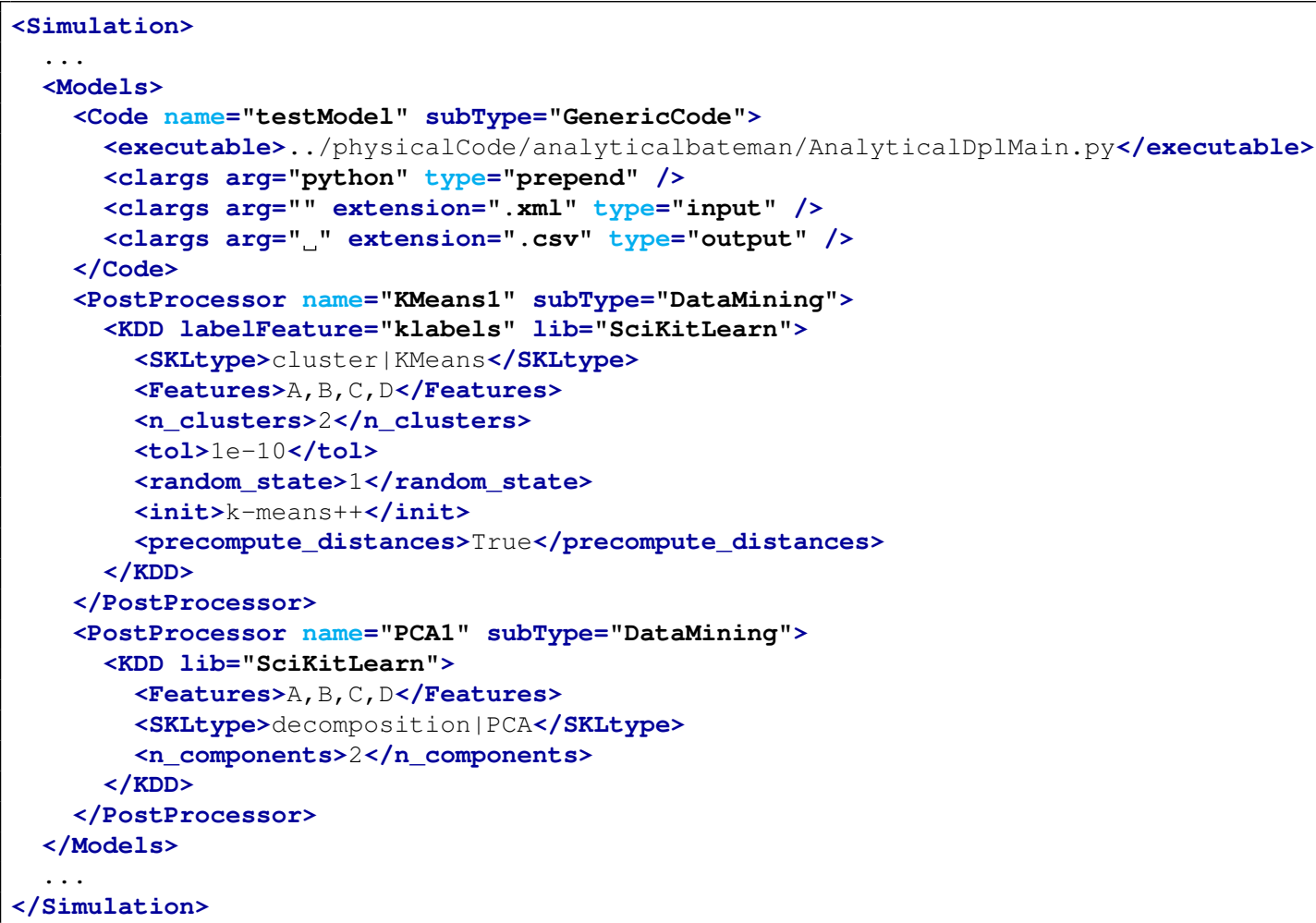

The goal of this example is to show how the data mining algorithms in RAVEN can be useful to analyze large data set.

In addition to the previously explained Code model, two Post-Processor models (DataMining $\mid$ cluster $\mid K M$ and DataMining $\mid$ decomposition $\mid P C A$ ) are specified. Note that the post-processing is performed on all the output FOMs used in this example ( $A, B, C$ and $D$ ). 


\section{Distributions:}

raven/tests/framework/user_guide/DataMining/dataMiningAnalysis.xml

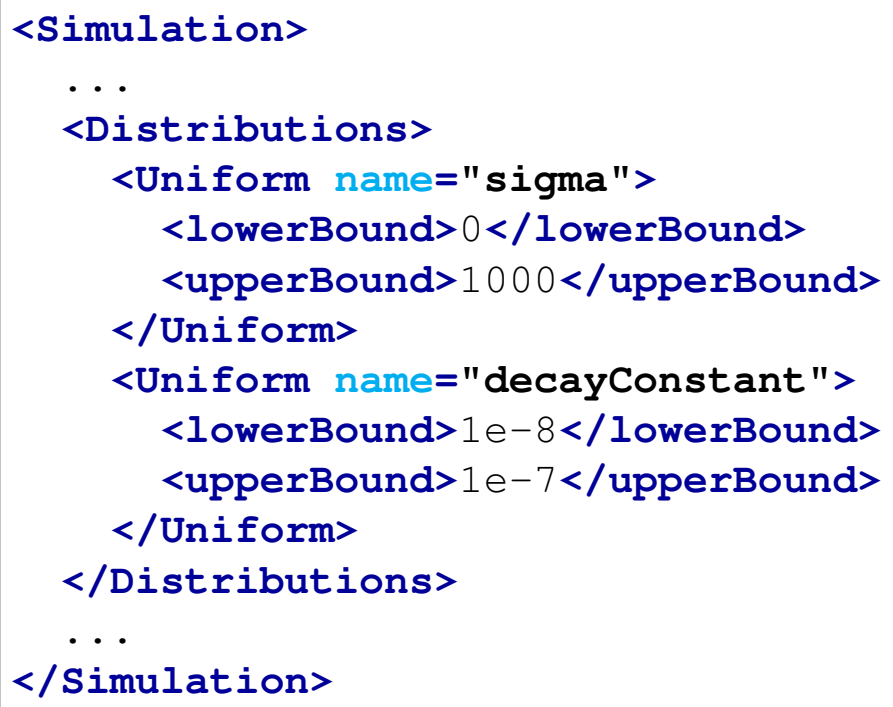

$</$ Simulation $>$

In the Distributions XML section, the stochastic model for the uncertainties are reported. In this case 2 distributions are defined:

- sigma $\sim \mathbb{U}(0,1000)$, used to model the uncertainties associated with the Model sigma$A$ and sigma-B;

- decayConstant $\sim \mathbb{U}(1 e-8,1 e-7)$, used to model the uncertainties associated with the Model decay- $A$ and decay- $B$.

\section{Samplers:}

raven/tests/framework/user_guide/DataMining/dataMiningAnalysis.xml

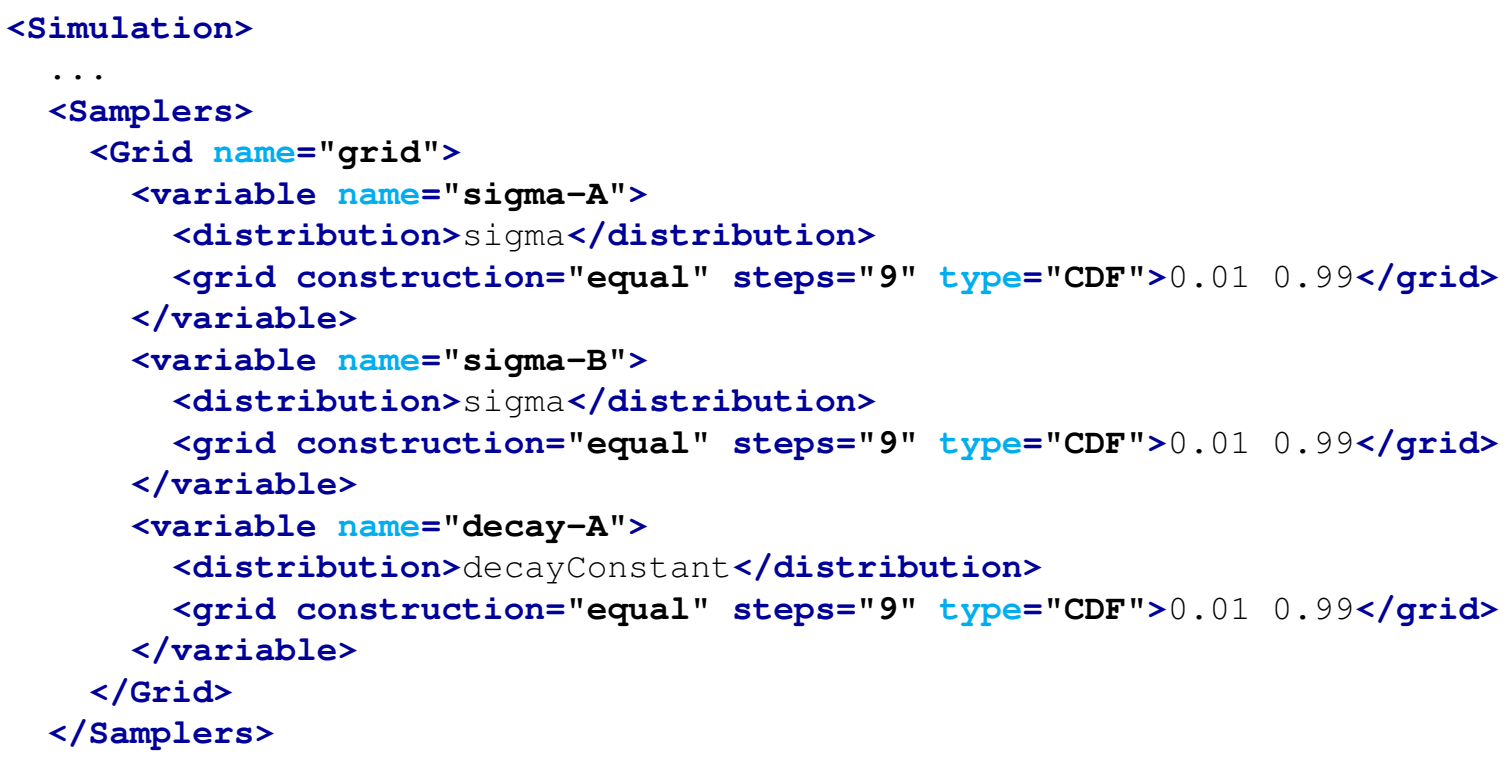


...

$</$ Simulation $>$

In order to obtain the data-set on which the data mining algorithms are going to be applied, a Grid sampling approach is here employed.

\section{DataObjects:}

raven/tests/framework/user_guide/DataMining/dataMiningAnalysis.xml

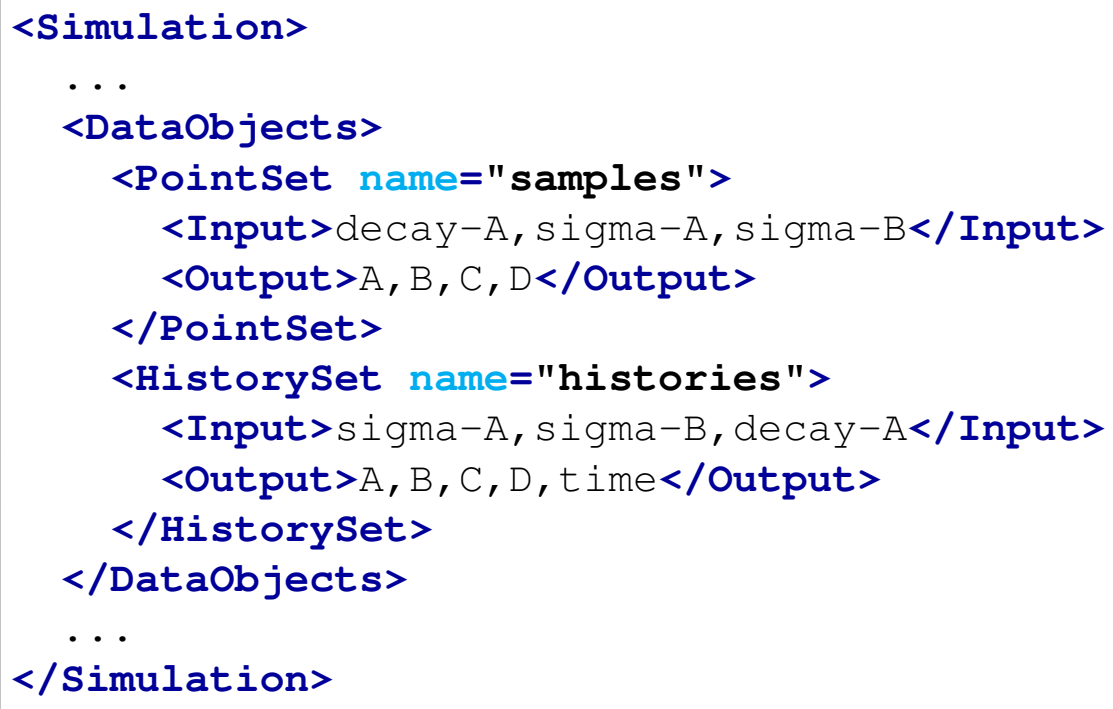

Int this block, two DataObjects are defined: 1) PointSet named "samples" used to collect the final outcomes of the code, 2) HistorySet named "histories" in which the full time responses of the variables $A, B, C, D$ are going to be stored.

\section{OutStreams:}




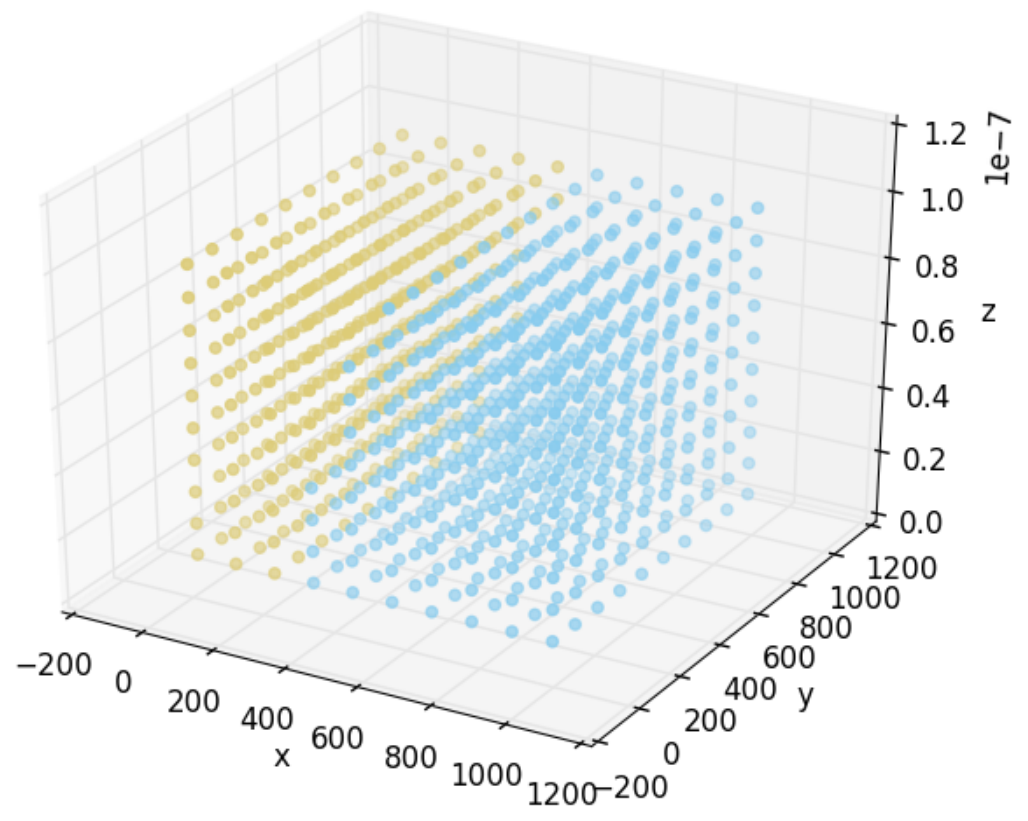

Figure 31. K-means clustering on original dataset.

raven/tests/framework/user_guide/DataMining/dataMiningAnalysis.xml

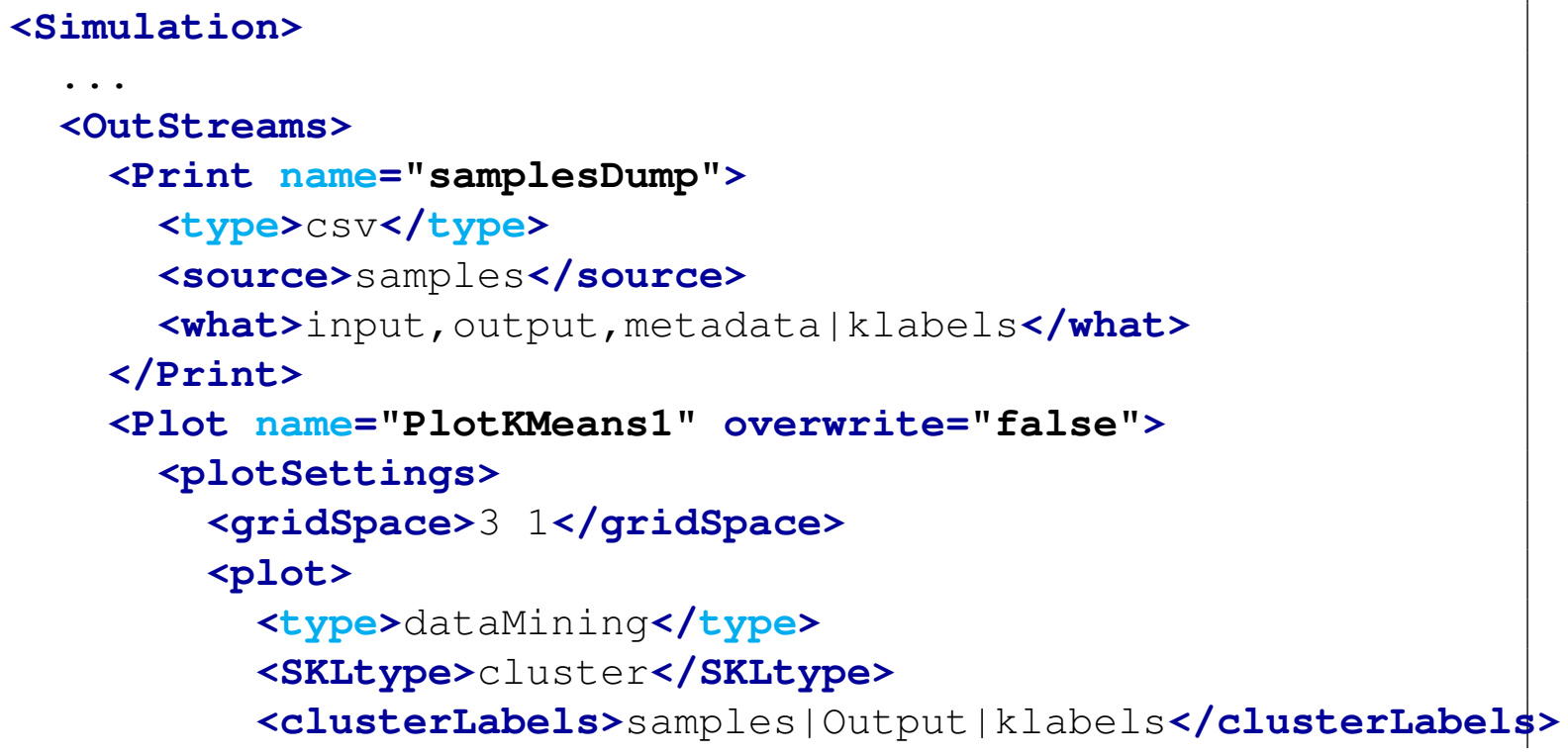




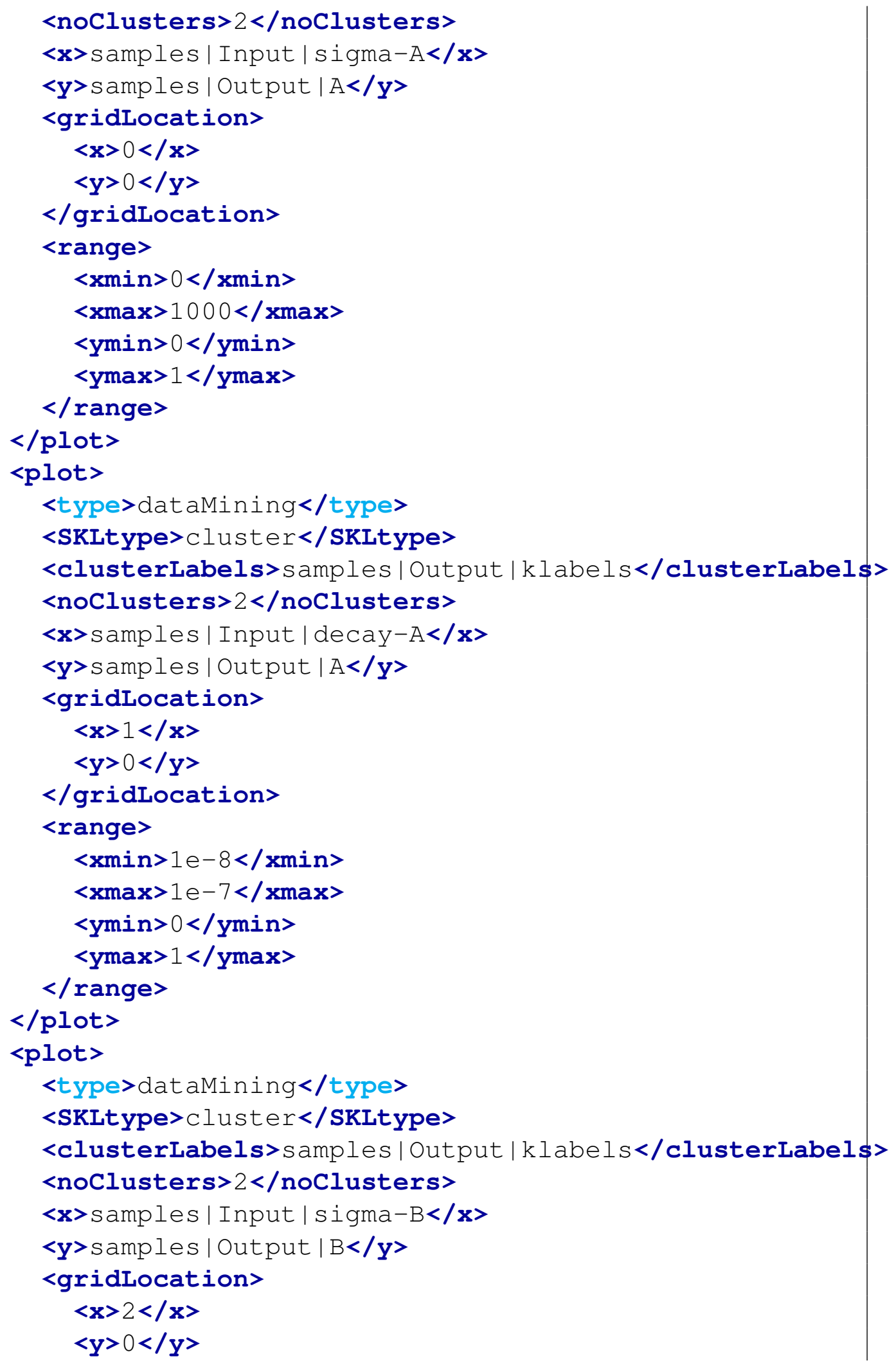




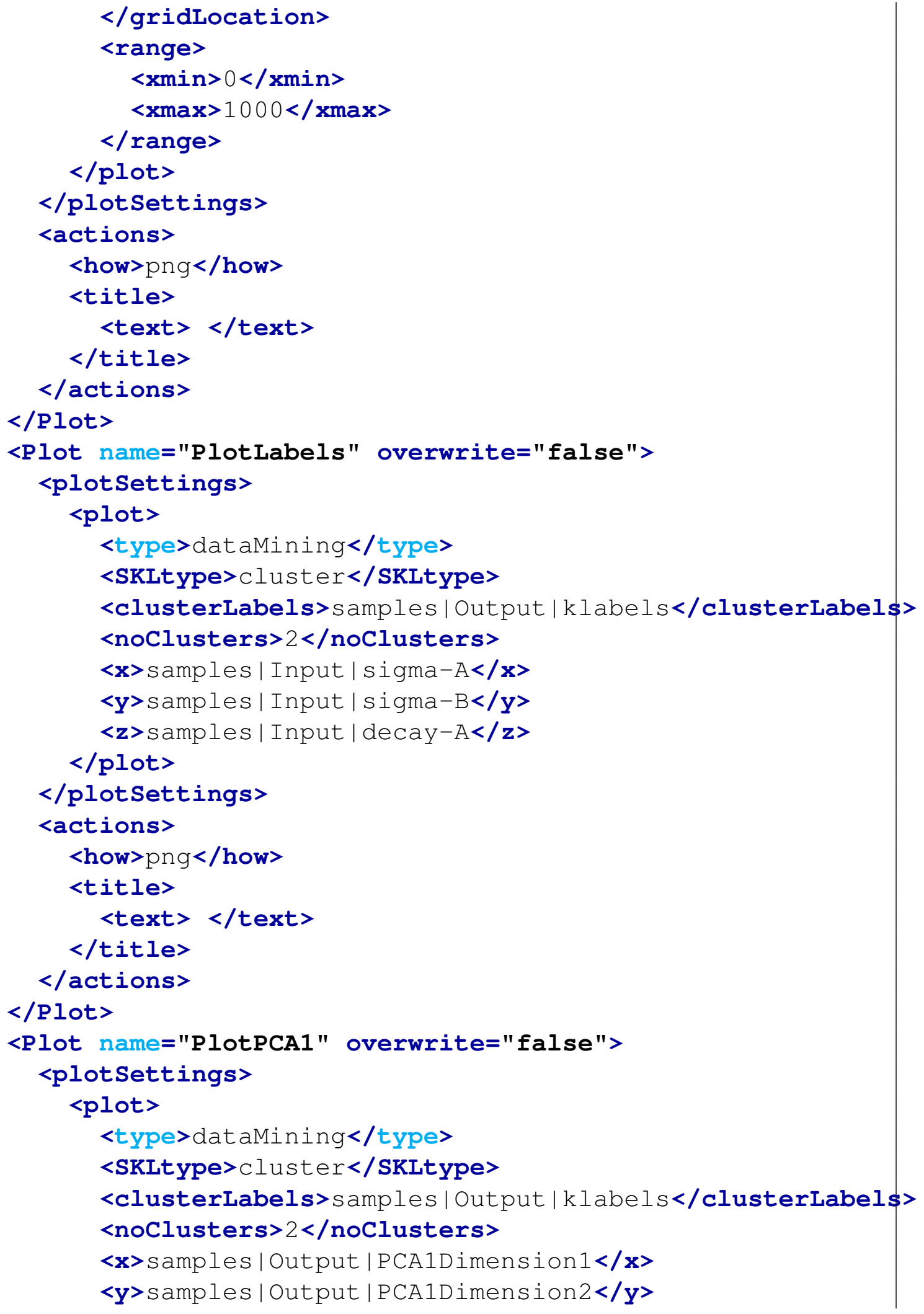




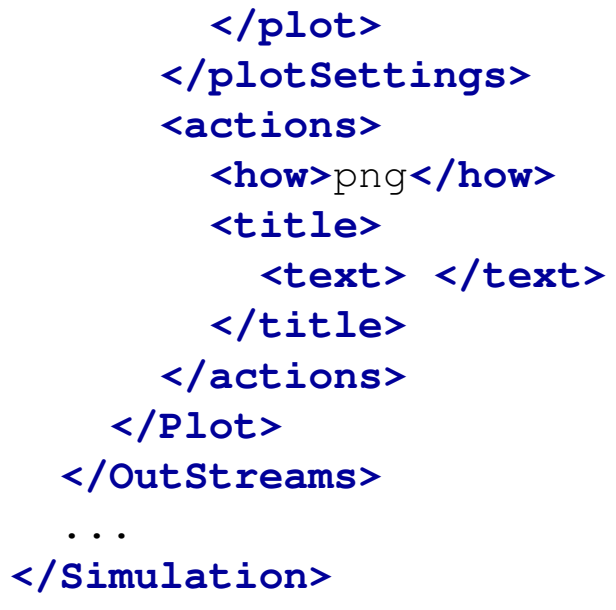

This workflow uses one Print OutStream and three Plot OutStreams:

- "samplesDump", which writes the original sample set with the additional columns from the PostProcess steps,

- "PlotKMeans1", which plots the samples against the Figures of Merit with coloring according to the KMeans clustering,

- "PlotLabels", which plots all the samples and colors them according to the KMeans clustering,

- "PlotPCA1," which plots the surrogate principal component dimensions and their associated clustering.

Note that a special kind of plot, the "dataMining" <type>, has been implemented to simplify plotting complicated results using RAVEN, and is used in all three of the plots in this workflow. Also note the use of the <range> block to define the data range of the plots created.

\section{Steps:}

raven/tests/framework/user_guide/DataMining/dataMiningAnalysis.xml

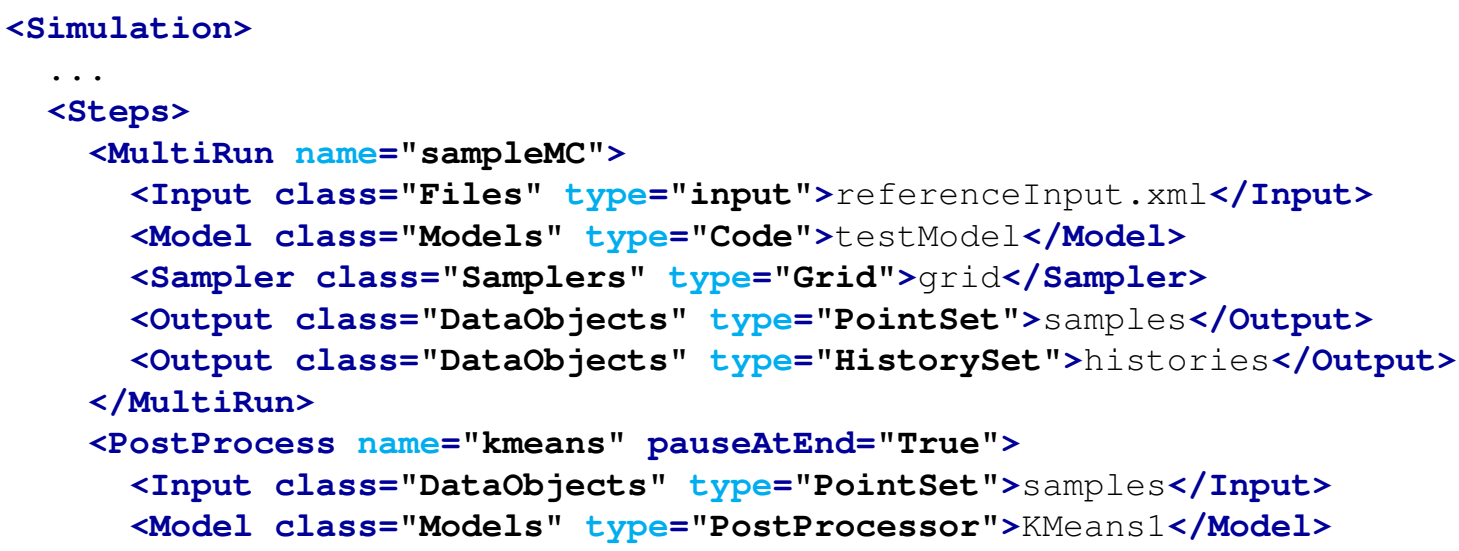




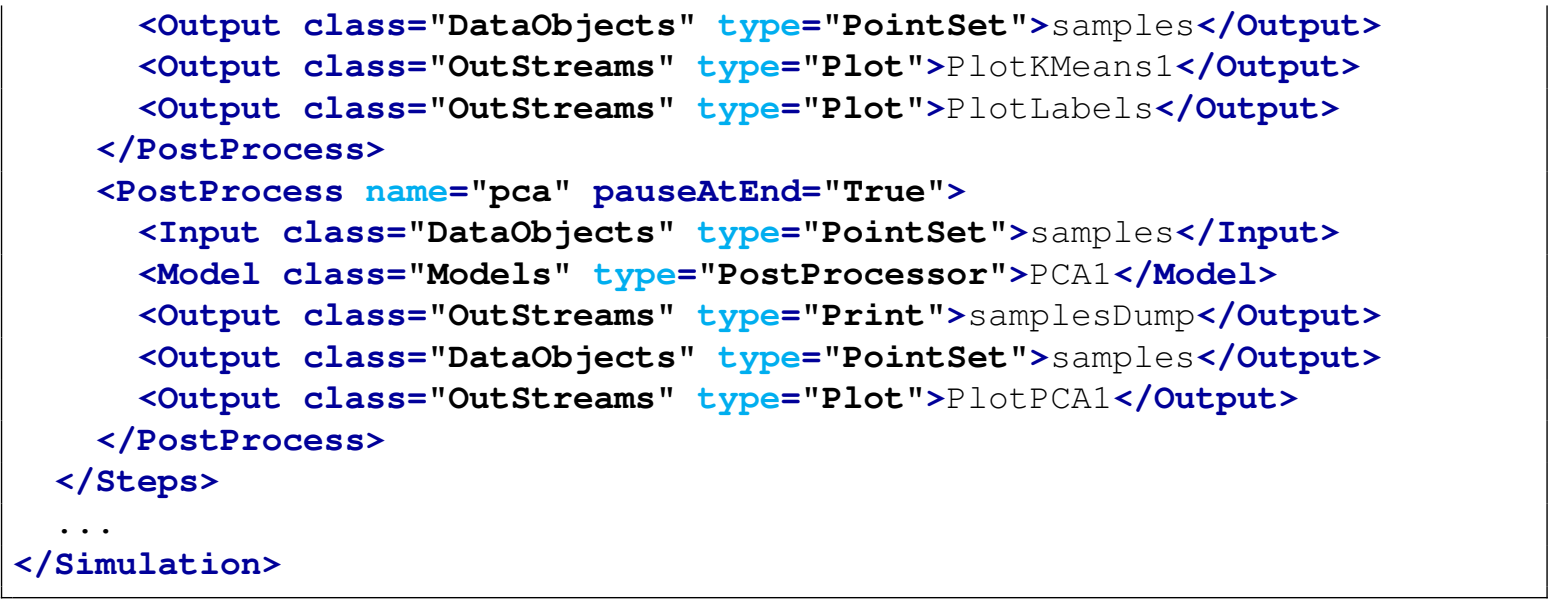

Finally, all the previously defined Entities can be combined in the <steps> block; 3 <Steps> have been inputted:

- <MultiRun> named "sample", used to run the multiple instances of the driven code and collect the outputs in the two DataObjects. The <Sampler> is inputted to communicate to the Step that the driven code needs to be perturbed through the Grid sampling strategy;

- <PostProcess> named "kmeans", used to analyze the data obtained through the sampling strategy. In this step, a K-Means algorithm is going to be employed, plotting the clustering results; Step that the driven code needs to be perturbed through the Grid sampling strategy;

- <PostProcess> named "pca", used to analyze the data obtained through the sampling strategy. In this Step, a PCA algorithm is going to be employed, plotting the decomposition results.

Figure 31 shows the clustering on the original input space.

Figure 32 shows the clustering on the projected input space. It can be noticed, that the algorithm fully capture the fact that the parameter sigma $-B$ does not impact the response $A$ (being completely independent).

Figure 33 shows the PCA decomposition on the data set. 


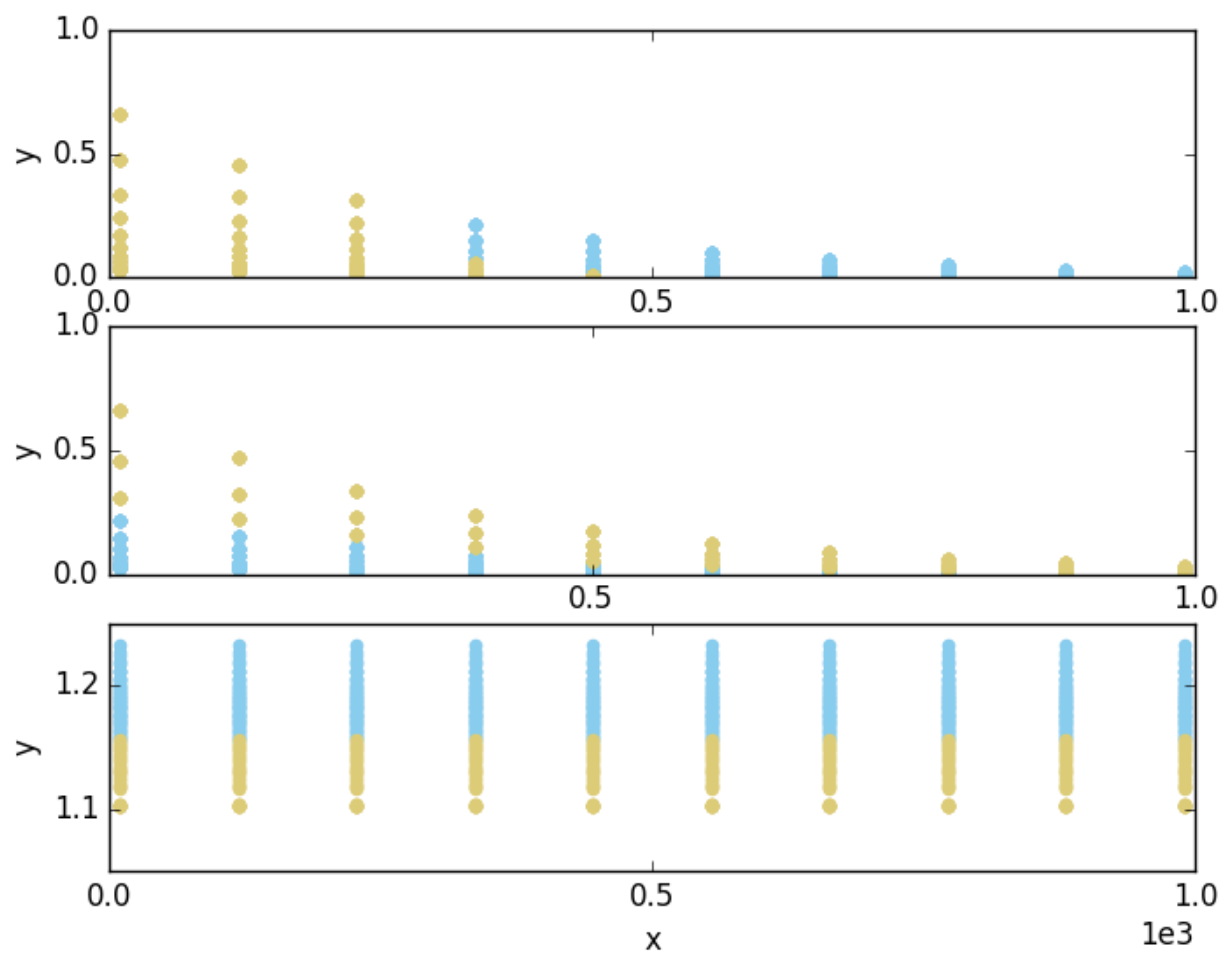

Figure 32. K-means clustering on projected parameters. 


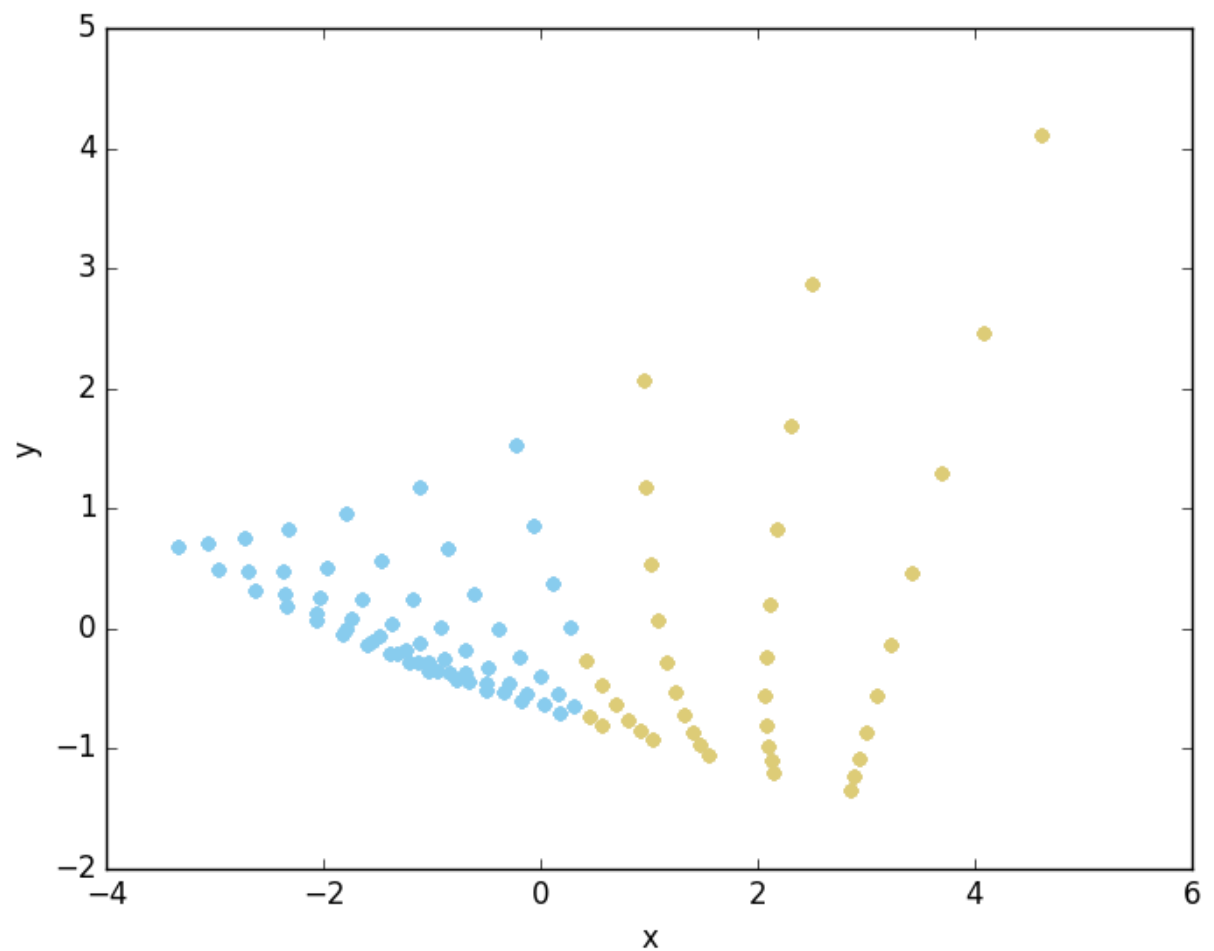

Figure 33. Principal Component Analysis. 


\section{Model Optimization}

When analyzing the range of values obtainable by a model, frequently a key question is "what set of parameters result in the best response value?" To answer this question, RAVEN uses the <Optimizer>, a powerful sampler-like entity that searches the input space to find minimum or maximum values of a response.

In the remainder of this section, we will explore how to use the optimizer using a simple analytic problem, with a two-dimensional input space and single response of interest. After getting used to running with the optimizer, we will add increasing complexity, including changing adaptive step sizes, initial conditions, parallel trajectories, input space subdivision, input space constraints, and response constraints.

To demonstrate the operation of the Optimizer entities in RAVEN, the model we consider is the Beale function, which is documented in the analytic tests for RAVEN and replicated here:

- Function: $f(x, y)=(1.5-x+x y)^{2}+\left(2.25-x+x y^{2}\right)^{2}+\left(2.625-x+x y^{3}\right)^{2}$

- Domain: $-4.5 \leq x, y \leq 4.5$

- Global Minimum: $f(3,0.5)=0$

The two inputs are the variables $x$ and $y$, and the response is a value we'll assign to ans, short for "answer". The model is an external model in RAVEN, and can be found at

raven/tests/framework/AnalyticModes/optimizing/beale.py •

The function's values are distributed as in Fig. 34.

Note that throughout this example we use the SPSA optimizer by way of demonstration, since it is the first advanced algorithm for optimization included in RAVEN; many of the options and parameters apply to other optimizers, and details can be found in the RAVEN user manual.

\subsection{Introduction: The Optimizer Input}

As with other entities, the Optimizer gets its own XML block in the RAVEN input. Here's an example of an input for a SPSA optimizer named ' opter' : 


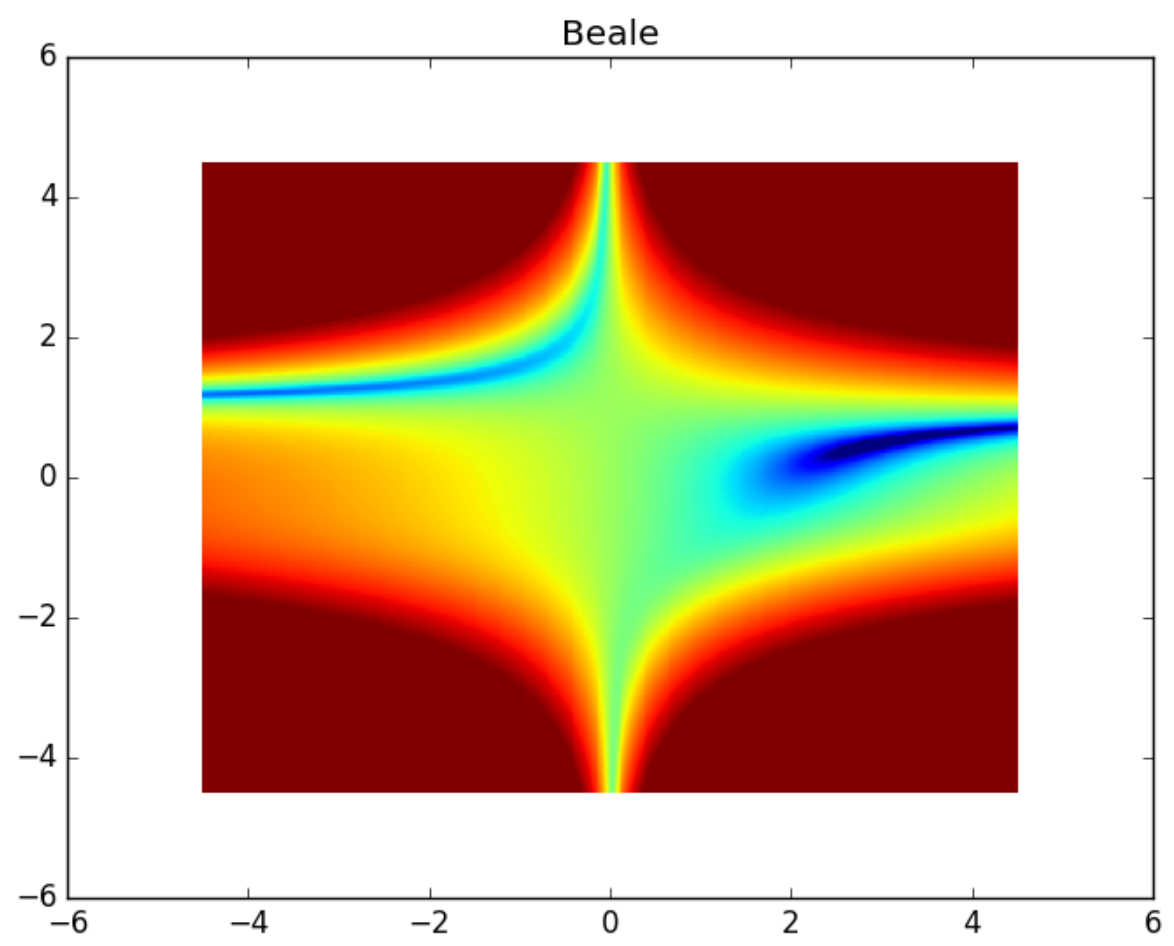

Figure 34. Plot of Beale's function for Optimization

raven/tests/framework/user_guide/optimizing/simple.xml

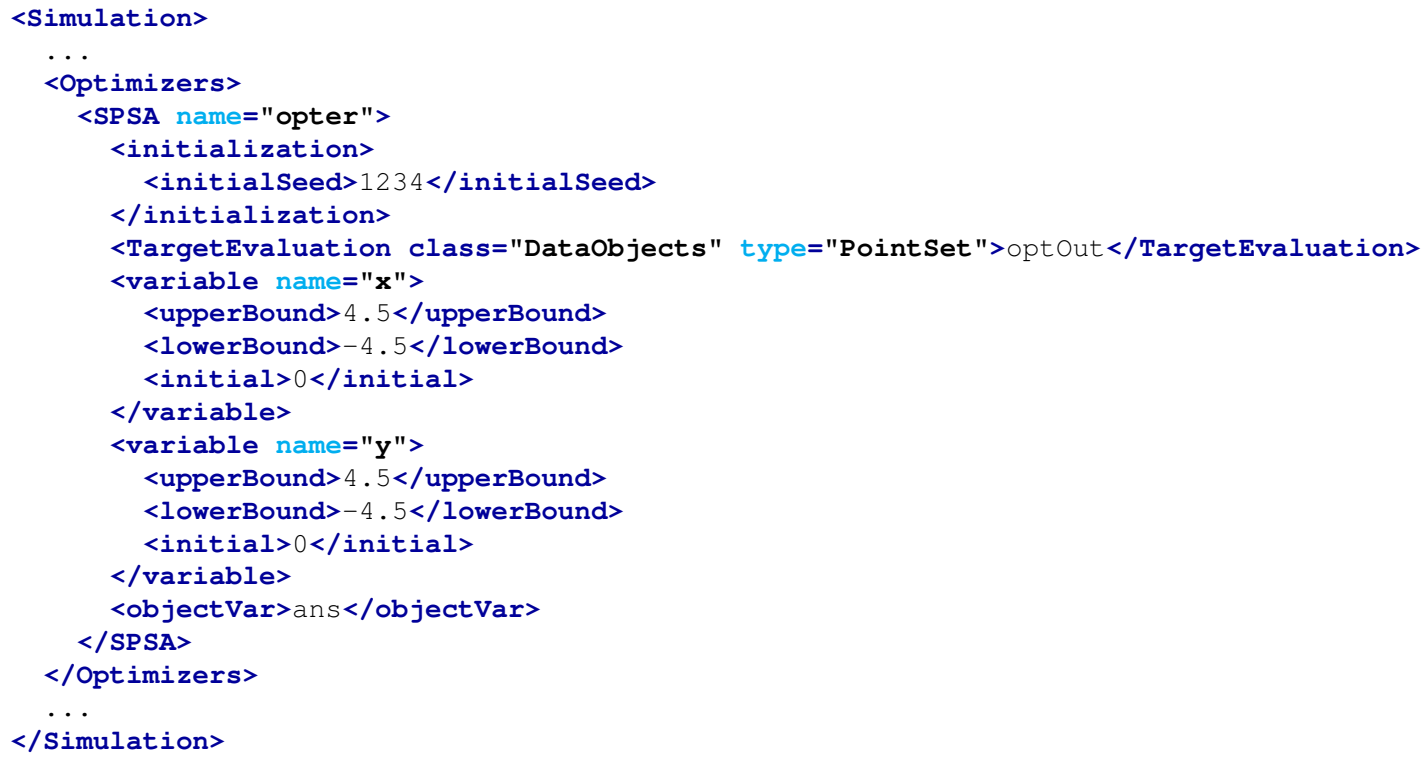


This is the smallest amount of input needed to run an optimization problem, with the exception that we include the <initialseed> to maintain consistent results. Note the required blocks included to define the optimizer:

- <TargetEvaluation>, which declares the DataObject that the optimization search evaluations are going to be placed in. All of the optimal points found as part of the optimization, as well as any other points evaluated as part of the algorithm, are placed in this object so the optimizer can retrieve this information later. When this data object is defined, it is critical that the objective variable is defined in the output space, and the input variables in the input space, so the optimizer can collect the results of its sampling. The data object type should be "PointSet" for this data object. In this example, we use the self-descriptive optOut data object.

- <variable>, which is where you can define the input space variables, one for each of these nodes. Declaring a variable here informs the optimizer that you want it to find the optimal value for this variable, along with the other variables declared in their own blocks. Additionally, you define the upper and lower bounds of the variable, which will give the optimizer some general expectations for finding the optimal point; it will never try to sample a value smaller than the lower bound or larger than the upper bound. In the example we define variables $x$ and $y$ as our input variables, and both of them coincidentally range between -4.5 and 4.5. We set the initial values for both variables to 0 through the <initial> block, which is required in most cases; the exception is when a preconditioner sets them in mulitlevel optimization, but we're not concerned with that feature at this point.

- <objectvar>, which is where you indicate the variable for which you want to find the minimum (or, if you change the default, maximum). As listed here, we want to minimize the value of ans given a range of possible values for $\mathrm{x}$ and $\mathrm{y}$.

The other critical blocks in this input are as follows:

\subsubsection{Models}

raven/tests/framework/user_guide/optimizing/simple.xml

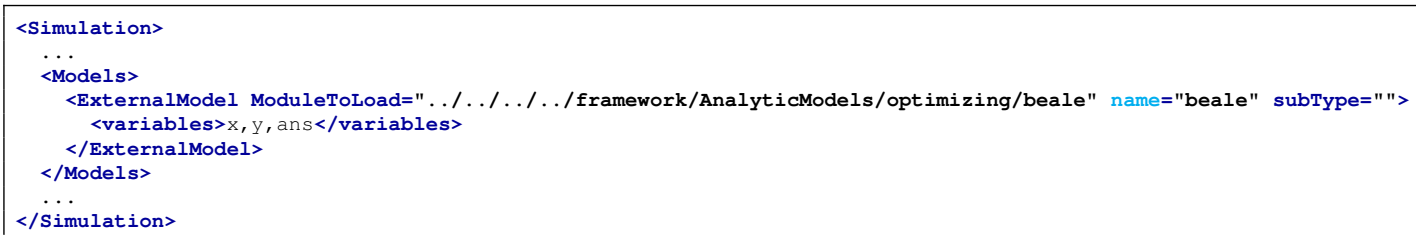

Note that we define the external model with the name 'beale' and provide a path to the analytic model itself. This model is set up with the run method that allows RAVEN to run the model. We also list all our input/output variables, $x, y$, and ans. 


\subsubsection{Data Objects}

raven/tests/framework/user_guide/optimizing/simple.xml

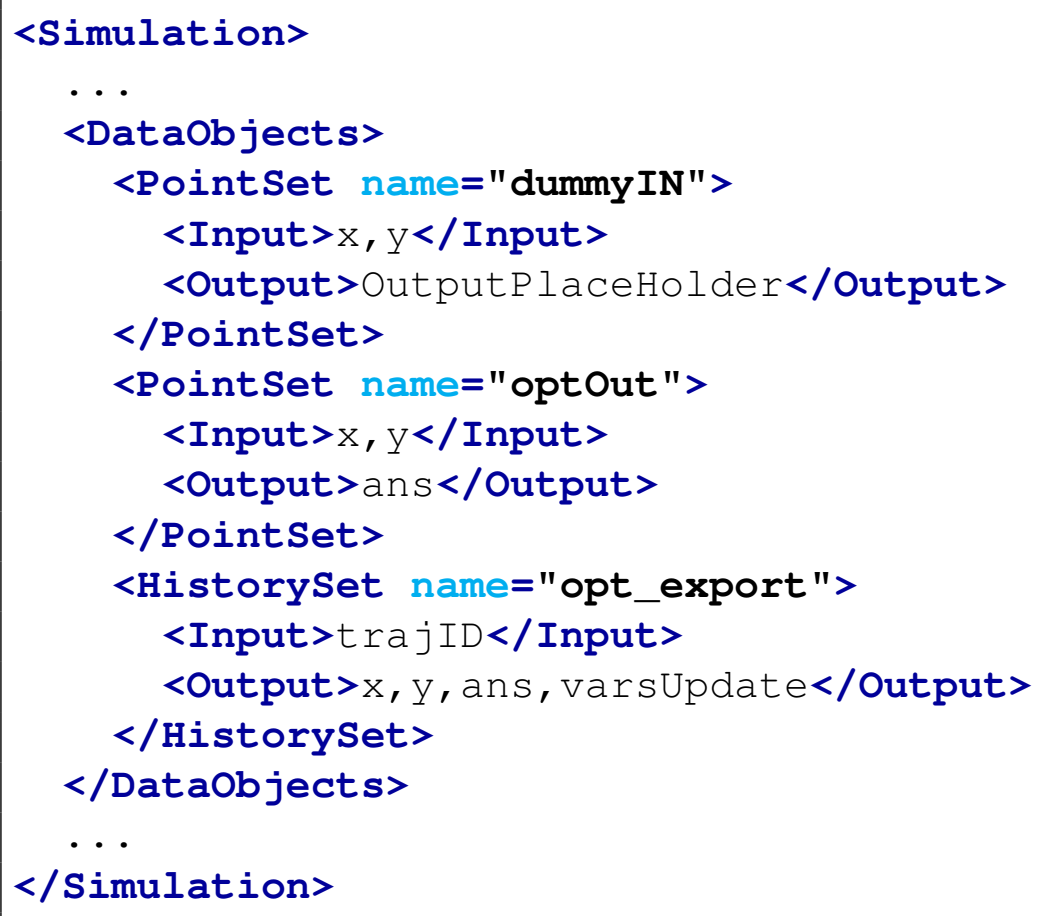

We have three data objects: ' dummy IN', which is necessary to define the input space of our external model in the Steps; ' optOut', which will hold all of the samples taken by our optimizer; and 'opt_export', which will hold the actual solution path taken by our optimizer. Note that while 'optOut' is a Point Set, 'opt_export' is a History Set. This is because we store the path travelled by the optimization algorithm as a history, with varsUpdate keeping track of the optimization steps taken. Note especially how the input of 'opt_export' is set to trajID, which is a special keyword for the Optimizer history tracking, as is the output variable varsUpdate. There are several other special keyword outputs that can be written to the Solution Export data object, that can be found in the user manual.

\subsubsection{Out Streams}

raven/tests/framework/user_guide/optimizing/simple.xml

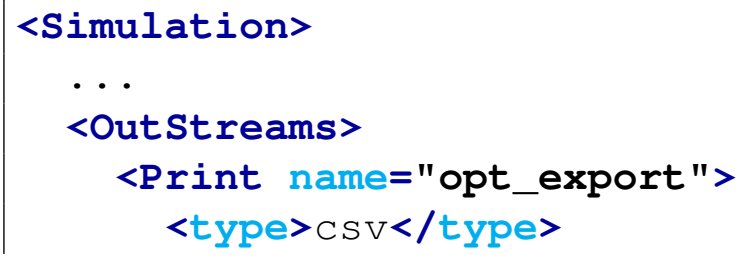




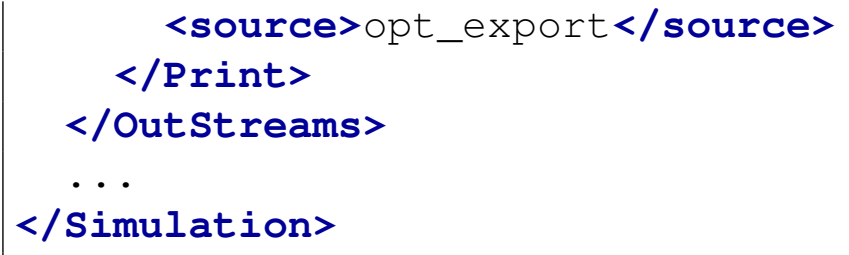

Here we define the way to print the output of our optimization algorithm. There's not much to note, except that we'll be printing the optimization path as a History-based CSV.

\subsubsection{Steps}

raven/tests/framework/user_guide/optimizing/simple.xml

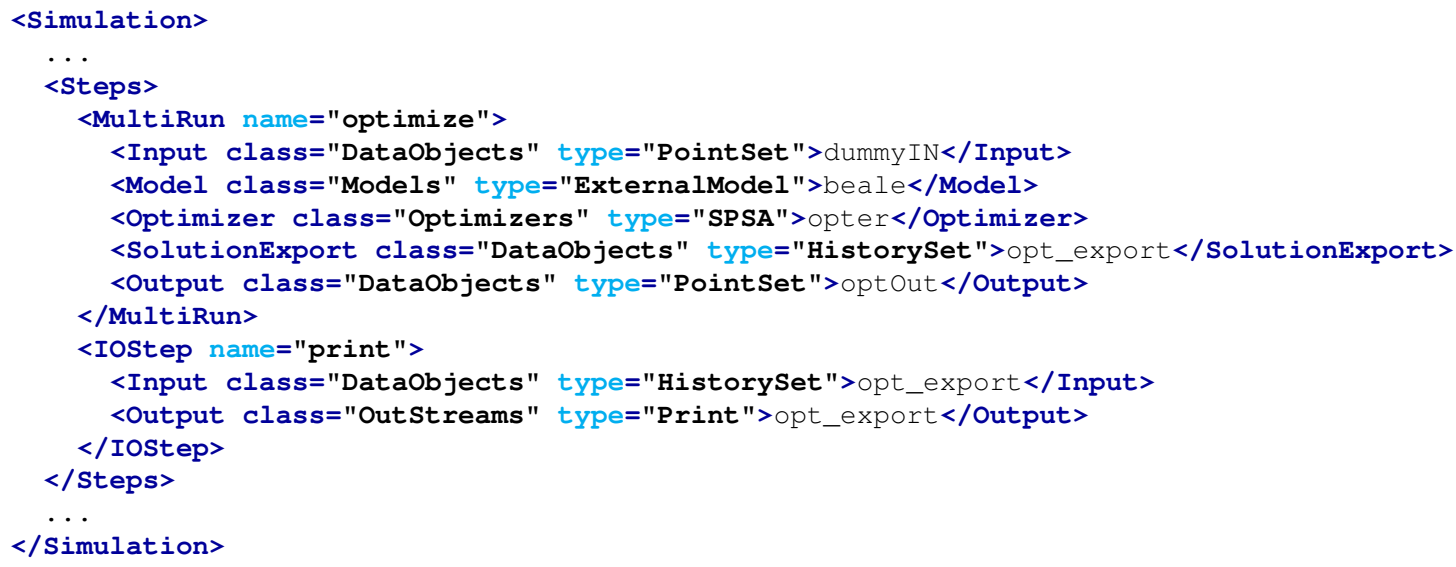

Here we put it all together into a work flow that RAVEN can follow. We only need two steps: one to optimize, and one to print out the results. To actually perform the optimization, we need a MultiRun step, which we cleverly name 'optimize'. For input we take the placeholder data object dummyIN, which sets up the input space of the model we defined, beal. Where a <Sampler> would normally go, we include the <Optimizer> we defined earlier. We output to the same data object we indicated in the Optimizer's <TargetEvaluation> node. Finally, we note specifically the use of the <SolutionExport > node. The data object defined in this node is where the Optimizer will write the optimization path history, with the final entry being the last step taken by the optimizer. The IOStep is unremarkable, used simply to write out the optimization path history to file.

\subsubsection{Conclusion}

After reviewing the components (don't forget the RunInfo block!), you can run this example and see the results. In particular, we can view the history of the optimizer in Simple/opt_export_0 . cSV. 
Note that opt_export is the name of the $<$ Print $>$ OutStream we defined in the input file, and the $\_0$ indicates this is the first optimization path followed (we'll cover multiple paths later in section 9.2).

When we open the file (preferably in a CSV reader such a spreadsheet viewer), we see a CSV with four headers, the outputs defined in the data object in the input file: $x, y$, ans, and varsUpdate (not necessarily in that order). $x, y$, and ans are the values of the variable at each optimization iteration, while varsUpdate gives the sequential order of the optimization iteration.

If we look at the last line, we converged around $f(1.61,-0.189)=1.67$, which is okay but still quite a ways from the analytic optimal point $f(3,0.5)=0$. If we look at the output from the run, we can look at the last time RAVEN was "Checking convergence for Trajectory 0". Below that statement, there are a series of convergence criteria and their status. We can see that the reason we converged at the end is the Relative Loss Diff, which means the relative change in the response ans was sufficiently small between steps to cause convergence. Clearly, we claimed convergence prematurely because of the default convergence values used by the optimizer. Because these convergence criteria are very problem-specific, the default parameters will not work best for all problems.

We can improve this result by changing convergence parameters as well as step size growth and shrink factors, all of which can be found in the user manual, and many of which we'll discuss in the rest of this section.

\subsection{Initial Conditions and Parallel Trajectories}

Notice we set the optimization search to start at $(0,0)$. You can change this initial value through the <initial> block within the <variable $>$ definition node.

Furthermore, RAVEN offers the possibility to run multiple optimization paths in parallel. Because many (perhaps most) optimization techniques get stuck in local minima, using multiple paths (or trajectories as they are called in RAVEN) increases the likelihood that one of the trajectories will find the global minimum point. You can request multiple trajectories by providing a variety of initial conditions in the <initial> block, as shown in this Optimizer example:

raven/tests/framework/user_guide/optimizing/multiple_trajectories.xml

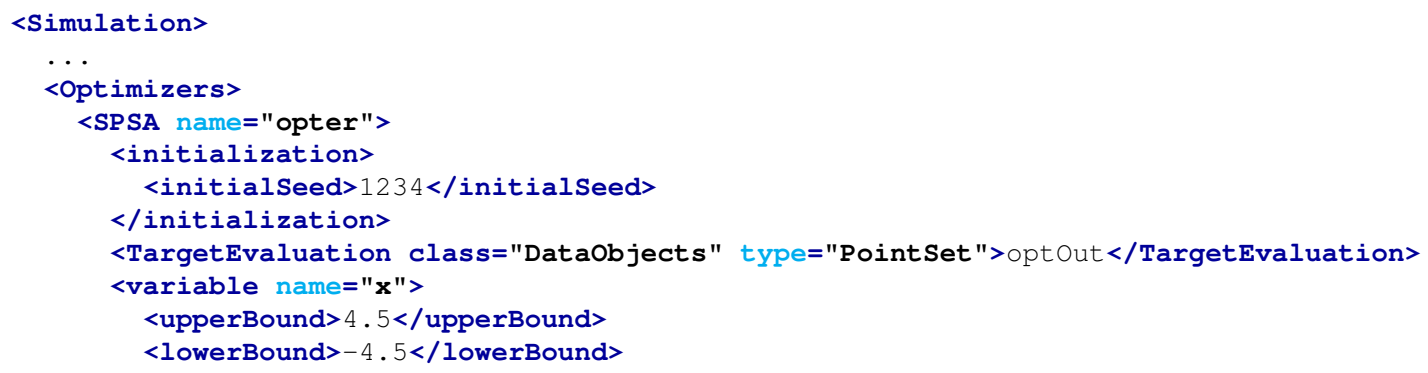




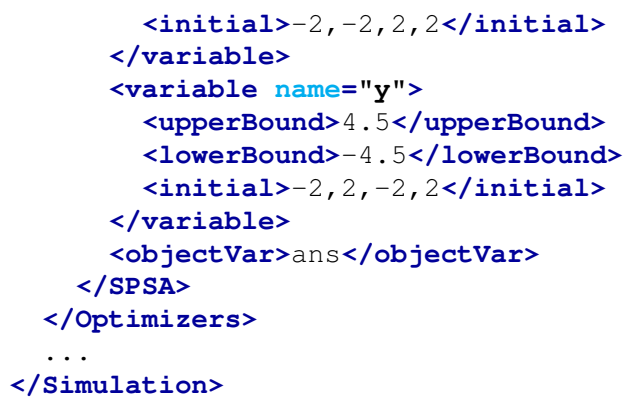

Note that the ordered pairs are split across the <initial> nodes, so that the first trajectory will start as a point made up of all the first entries, the second trajectory starts at all the second entries, and et cetera. In this case, we've requested starting points at $(-2,-2),(-2,2),(2,-2)$, and $(2,2)$. This (and defining a new working directory in the $<$ RunInfo $>$ block) is the only input change between the original file and this one.

When run, we can see the results in the working directory MultipleTraj. There, we see the same files as for the base case, plus opt_export files 0-3 instead of just 0. Each of these corresponds to the path that one of the initial points started at, as you can see at the top of each of these CSV files. We can see that trajectory 3 (who started at $(2,2)$ ) ended close to the analytic optimal point, while trajectory 0 was far from it.

In order to see a summary of the end point of each trajectory, we can use the additional RAVEN step described in 9.3 .

\subsection{Summarizing Results}

While seeing the histories of the optimization paths taken in the <SolutionExport> is informative, sometimes we just want to see the final optimal point found by each trajectory. In this case, we can use an InterfacedPostProcessor called HistorySetSnapShot to get the last point found by each optimizer. The postprocessor looks like this:

raven/tests/framework/user_guide/optimizing/summary . xml

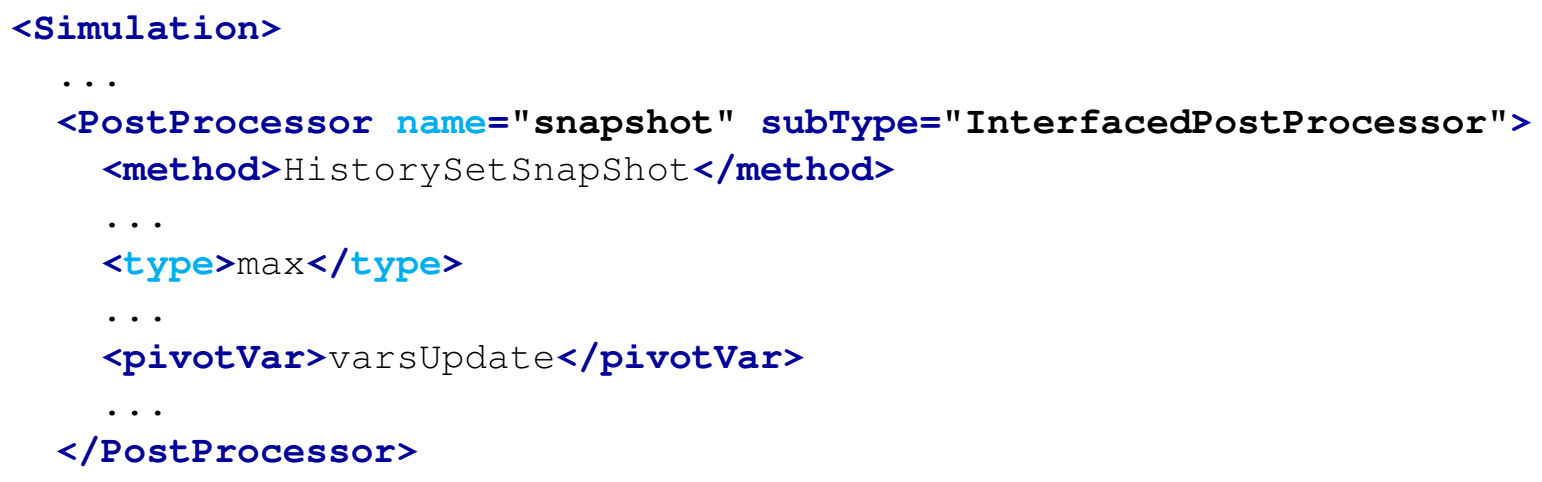


...

$</$ Simulation $>$

Note that we named the postprocessor ' snapshot', and instructed it to look for the ' $\max$ ' value of 'varsupdate' in each of the histories, and combine them into a single data set. We add the following Step to our <Steps > to run it:

raven/tests/framework/user_guide/optimizing/summary .xml

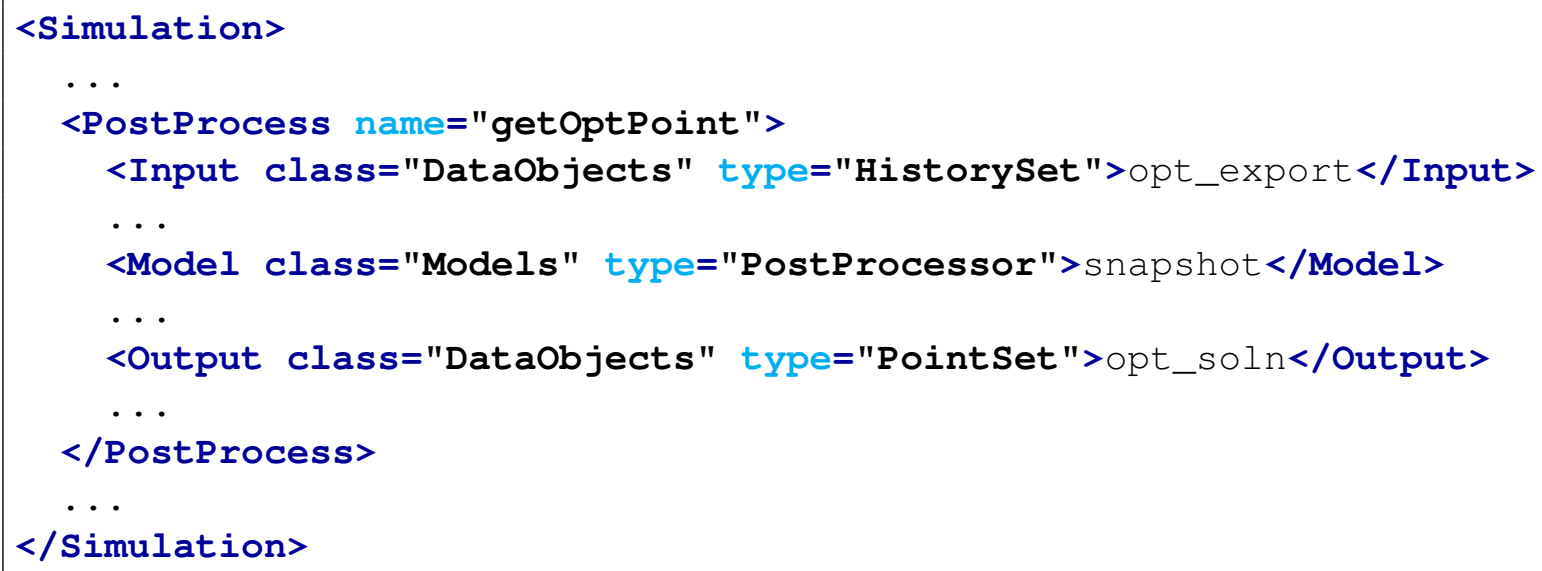

Don't forget to add 'getoptPoint' to the <Sequence> in the <RunInfo> block, before 'print' but after ' optimize'. Note that this post-processing step takes the <SolutionExport> data object from the optimizing step as input, runs the postprocessing model, then places the results in a new data object, ' opt_soln', which we add to the $\langle$ DataObjects $>$ list in the input file.

Note also that we added the printing of the 'opt_soln' data object to the 'print' <IOStep>; we only needed to write an <OutStream> for it, not a whole new step.

After running this input, you can open Summary/opt_soln. CSv to see the final results of each optimization trajectory. The variable trajID gives the trajectory label, $x$ and $y$ give the final location of the optimal point found by that trajectory, ans gives the value of the response at that location, and varsUpdate shows how many iterations were necessary to find that optimal point. The trajectory with the lowest value for ans is the most optimal point found.

\subsection{Adjusting Adaptive Steps}

As we've seen, some of the optimization paths are struggling to converge to meaningful optimal solutions. One way to improve this is to tinker with the convergence tolerances defined in the user manual. Another is to change the step size modifications used as part of the search process, which we discuss in this section. First, we briefly discuss how the SPSA chooses its step size, so we can make informed choices about what parameters to use. 
Because SPSA is a gradient-based method, it operates by starting at a particular point, estimating the gradient at that point, then taking a step in the opposite direction of the gradient in order to follow a downhill path. It adaptively chooses how long of a step to take based on its history. If the gradient is in the same direction twice in a row, the algorithm assumes there's further to travel, so increases its step size multiplicatively by the grainGrowthFactor, which by default is 2.0 in RAVEN. If, on the other hand, the gradient switches directions, then the step size is divided by the gainShrinkFactor, which again is 2.0 by default in RAVEN. This means that by default, if the gradient keeps going in the same direction, you always double your step size, while if you're bouncing back and forth in a valley, the step size is halved at each iteration.

By way of note, in higher dimensions, the actual growth or shrink multiplier is scaled by a dot product between the two previous gradients, with a max of the grain growth factor when the dot product is 1 (exactly aligned) and a minimum of grain shrink factor when the dot product is -1 (exactly opposite). This means if the gradient is at right angles with the past gradient, then the step size remains unchanged (dot product is 0 ).

There are some additional considerations for the step size change, as well. If the algorithm takes a step, then discovers the new point has a worse response value than the point it's at, it will reject the new point, re-evaluate the gradient, and flag the step size to be divided by the gain shrink factor. Because of this, if the gain shrink factor is too large, false convergence can be obtained when the algorithm struggles to find a new downhill point to move to. As a result, in practice it is often beneficial to have a gain shrink factor that is smaller than the gain growth factor.

For this particular example, we use gain growth factor of 1.5 (meaning when the gradient continues in the same direction our step grows to $150 \%$ of its old value) and a gain shrink factor of 1.25 (meaning when the gradient flips directions our step size shrinks to $80 \%$ of its old value). We add this to the base case (simple.xml) to get:

raven/tests/framework/user_guide/optimizing/step_size.xml

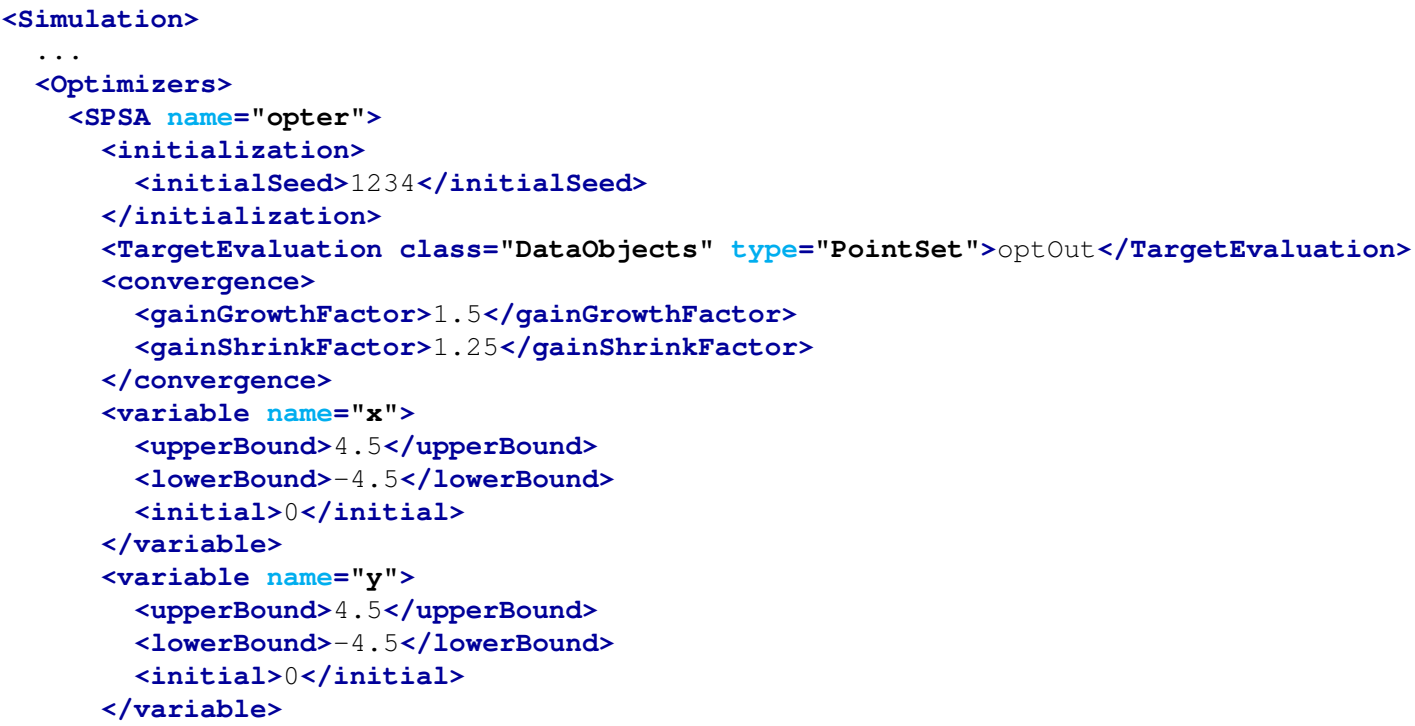




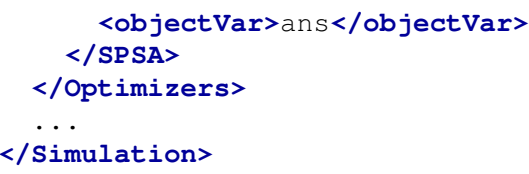

Note the definition of the gain growth and shrink factors in the <convergence> block. Reviewing the output file StepSize, we can see more steps were taken than the case using default step sizes, but the final solution was $f(2.65,0.403)=0.0286$, which is much closer to the analytical solution of $f(3,0.5)=0$ than the base case and within our convergence tolerances of the right solution.

It is often challenging to find the best gain growth and shrink factors, and these can have a very significant impact on the speed and accuracy of the convergence process. Too large a shrink factor results in poor resolution of valleys, while too small a shrink factor results in many unnecessary evaluations of the model.

\subsection{Denoising Stochastic Problems}

While many of the models we see in optimization are deterministic (meaning running the same inputs into the model yields the same results every time), there are quite a few stochastic models that we might desire to optimize. For example, a model that included Brownian motion or is solved using unseeded random numbers might be considered stochastic.

The difficulty with optimizing noisy problems rests in the unreliability of a single sample. If we send a single set of inputs into a stochastic model, we can't trust the results to be consistent. One way to measure the consistency of the results is through a signal-to-noise ratio (SNR). There are many ways to define this value; for our purposes, we will use the ratio of the mean of the signal to the standard deviation of the signal, $\mu / \sigma$.

To obtain an approximation of your SNR, you can use RAVEN to perform a Monte Carlo run on your model and then use the BasicStatistics postprocessor to collect the mean (expectedValue) and standard deviation (sigma) of your response. It's important to make this sampling all at a single value in the input space, so replace your variables with constants in the Sampler input. Once you have the mean and sigma, you have an idea of how noisy your model is. A SNR of 1 means the signal is just as big as the noise, making it very difficult to optimize. A SNR of less than 1 means the noise dominates the signal, and will make optimization almost impossible without introducing denoising. A SNR of more than 1 indicates the signal is stronger than the noise, and perhaps denoising is not necessary. If your standard deviation is 0 , then you don't have any discernable noise!

To denoise a model in RAVEN SPSA optimization currently, we turn our attention to the <Optimizer> subnode <parameter>, specifically the <numGradAvgIterations> node. 
This parameter instructs RAVEN to perform multiple gradient evaluations, including multiple evaluations of each optimal point, and use the average to make decisions in optimization pathing. By default, RAVEN takes one optimal point and one neighboring point to evaluate a gradient. Increasing the <numGradAvgIterations> will increase the number of times the optimal point is sampled, and how many neighbor points are sampled. This serves to denoise the model.

However, this also raises the question, how many resamples do I need to denoise my model? In a Wilks-like approach, we want to reduce the size of the confidence interval for our mean to be less than the noise. The number of resamples required depends on the size of the confidence interval $z$ and the confidence-to-noise ratio $\xi$ we want to ultimately have for the optimization algorithm. We also assume the distribution of the response is roughly Gaussian Normal, which may not be the case. The approximate equation for assuring the confidence interval is smaller than the noise is

$$
\begin{gathered}
\frac{z \sigma}{\sqrt{n}} \leq \xi \sigma, \\
n \geq\left(\frac{z}{\xi}\right)^{2} .
\end{gathered}
$$

Thus, the number of resamples depends on the confidence level as well as the desired ratio of the confidence interval to the noise.

A few values for varying ratios are given in Table 7 for the $99 \%$ confidence level $(z=2.576)$. That is, if you want the noise and confidence interval to have the same magnitude, only 7 resamples

\begin{tabular}{cc} 
Confidence-to-noise $\xi$ & Resamples necessary \\
\hline 1.0 & 7 \\
0.9 & 9 \\
0.7 & 14 \\
0.5 & 27 \\
0.1 & 664 \\
0.05 & 2655
\end{tabular}

Table 7. Estimate of the number of samples necessary to denoise models to varying confidence levels

are required. If, on the other hand, you want the confidence interval to be half the level of the noise, 27 resamples are required.

Note these are only guidelines; individual models may behave differently and require more or less resamples to provide a clear optimization path. 


\subsection{Input Space Subdivision}

In higher-dimensional problems, sometimes the input space can be divided into characteristic subspaces. For example, potentially one set of inputs vary slowly with consistent gradient directions in the response, while for another set of inputs the response fluctuates frequently with many local minima. In this case, it might be beneficial to optimize the subspaces in nested loops, slowly converging the smoothly-varying space while often converging the undulating space.

For these cases, RAVEN provides multilevel operation. To transition our base case to a multilevel case, we change the optimizer as follows:

raven/tests/framework/user_guide/optimizing/multilevel.xml

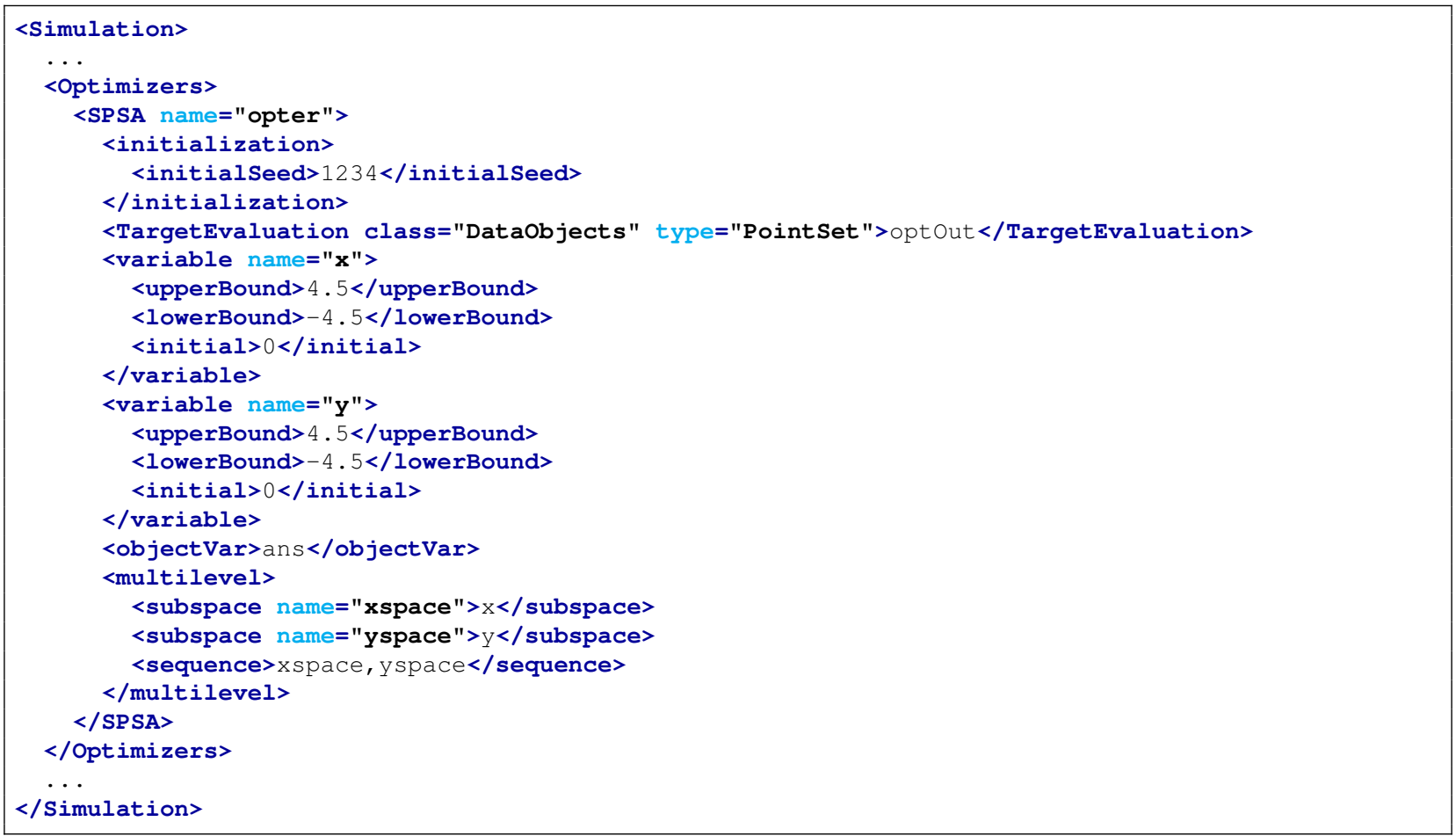

Note the addition of the <multilevel> node. In this node, we need to define the subspaces, then the ordering of the subspaces in terms of convergence priority. We intuitively name the subspace with $x$ the xgroup and similarly with $y$. Note that while in this example each subspace consists of only one variable, in general many variables may exist in any given subspace. Note also that it's a bad idea to include a variable in multiple subspaces.

The <sequence> can be thought of as an order of nesting optimization, where the first subspace listed is optimized most slowly (but only once) and the last is optimized frequently and swiftly. Each time a new optimal point is found, the innermost space (in our example yspace) is converged completely, holding all the variables in all other subspaces (e.g. xspace) constant at their most recent value. Once yspace is converged, we take a single step in the subspace xgroup, 
then return to converging yspace again. Every step in xspace triggers a full convergence of yspace.

In the results in Multilevel/opt_export_0.Csv, you can observe how first one dimension then the other is perturbed in the search for the optimal point.

It should be noted as well, using multilevel optimization does not necessarily improve the optimization path; in fact, in many cases, the number of model evaluations increases significantly for little gain in final optimal value. Multilevel is most useful when clustered subspaces share a particular feature in optimization that is useful to isolate from the rest of the optimization procedure, or if some subsets of inputs act independently from others in affecting the response.

\subsection{Functional Constraints}

Sometimes an optimization problem has a constrained input space, possibly where there is a tradeoff between two inputs. In this event, RAVEN allows the user to define a constraint function, which will cause RAVEN to treat this constraint as it would a boundary condition.

For example, we will introduce a void in the input where we reject inputs. This void is defined by rejecting all samples within $(x-1)^{2}+y^{2}<1$. We'll also include the modified step growth and shrink parameters discussed in section 9.4 .

To include a constraint function, we first have to define it in the RAVEN input as a $<$ Function $>$ entity:

raven/tests/framework/user_guide/optimizing/constrain.xml

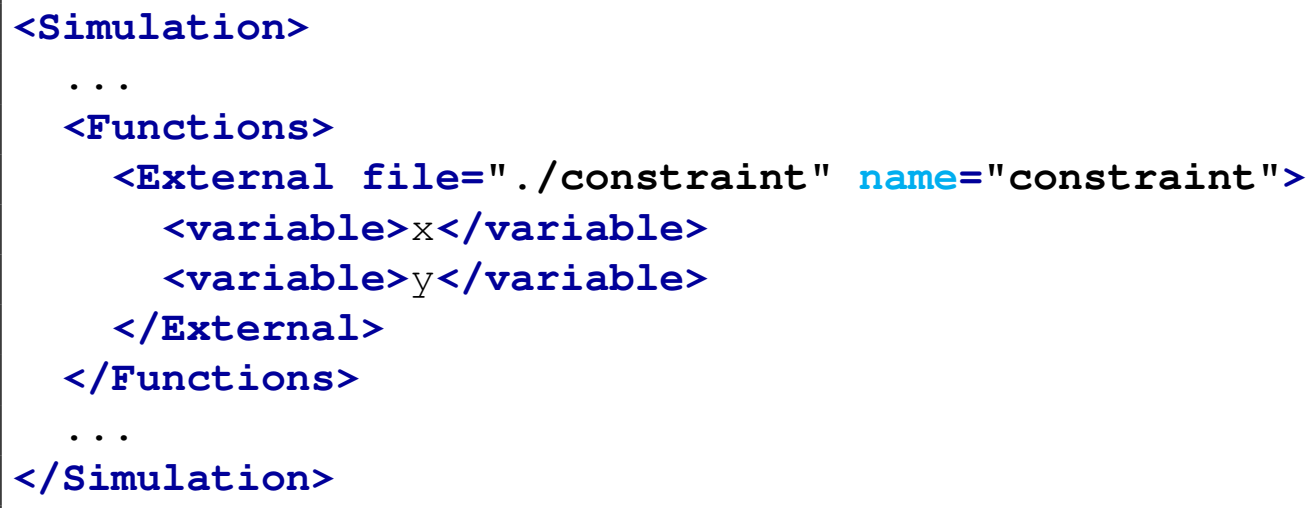

Note that the file . / Constrain/constraint.py is located relative to the working directory. Currently, external functions are always Python files. In that file, note that the only method is constrain, which is RAVEN's keyword to find the constraint function. RAVEN will pass in a self object, which will have the function variables defined in the $<$ Functions $>$ input available as members. The method constrain then returns a boolean which is True if the evaluation does not violate the constraint, or False if the constraint is violated. 
To attach the constraint to the optimizer, simply add it as an assembled <Function $>$ :

raven/tests/framework/user_guide/optimizing/constrain.xml

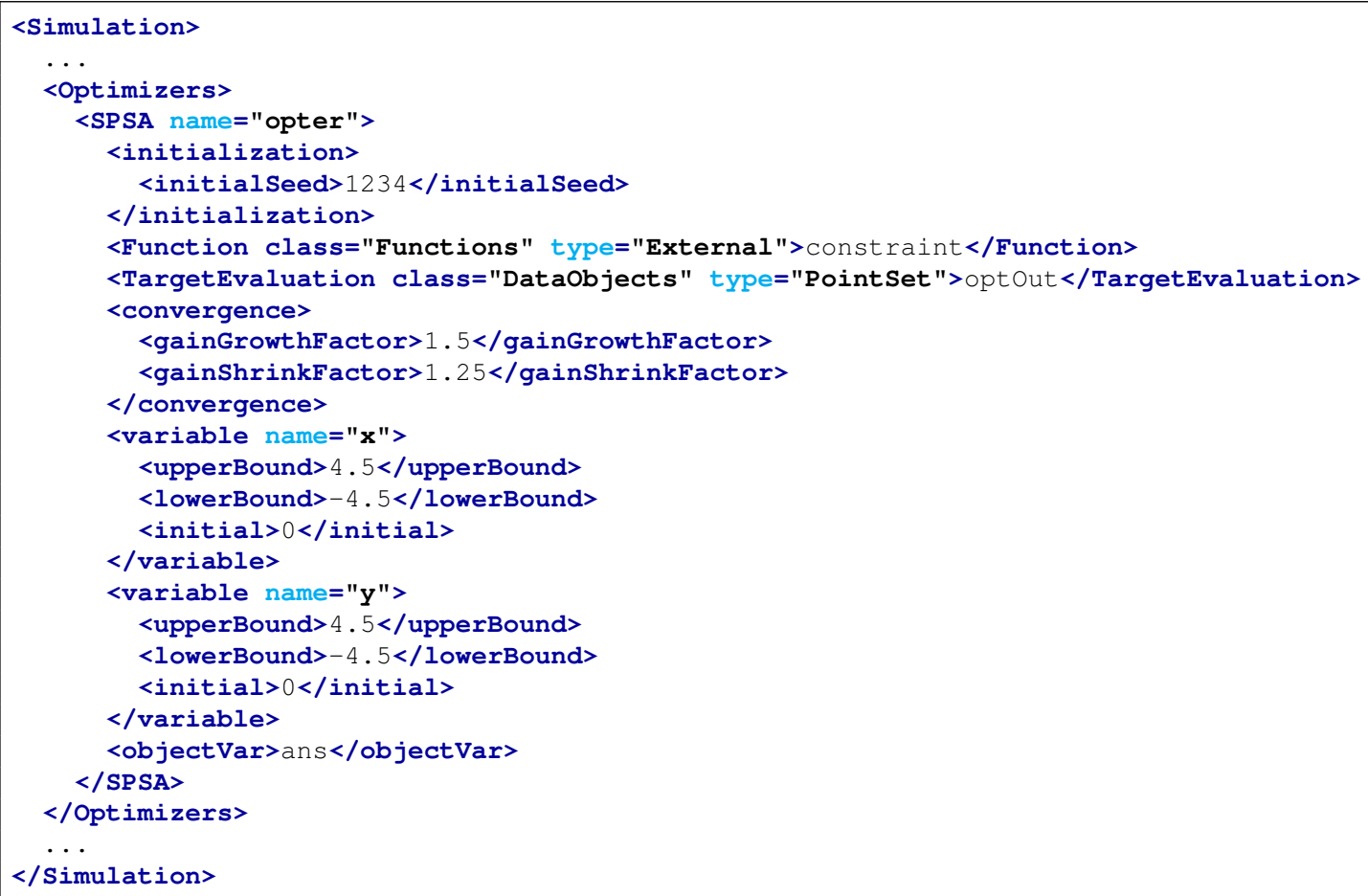

After running, looking through the path followed by trajectory 0 shows that instead of following the path from section 9.4, the path moves to lower $y$ values before swinging back up toward the optimal point. 


\section{EnsembleModel}

In most RAVEN MultiRun steps, a sampler provides a variety of inputs to a model, whose outputs then produce a set of responses that can be used in many ways. However, sometimes a single model is insufficient to produce the response we want. When this happens, the EnsembleModel opens up a new vista of simulation options.

The EnsembleModel is a powerful tool for chaining multiple models together, with outputs of some models becoming inputs for others. Whether this is adding a preprocessing or postprocessing model, or linking multiple physics-based models together, the EnsembleModel provides the functionality to see many models together as a single model in a MultiRun step.

Note that the EnsembleModel combines multiple models for each sample; that is, the whole ensemble of models is run for each sample taken by RAVEN. If instead you want to postprocess a batch of sampled data, try the PostProcessor models instead.

\subsection{Introduction: The EnsembleModel}

The key to the EnsembleModel is careful definition of the inputs and outputs of each model. When an EnsembleModel is defined in a RAVEN run, RAVEN will automatically scan the inputs and outputs of each model and determine the right order to evaluate the models in (or graph).

\subsection{Example: ballistics and impact}

As a basic example, consider two physics models. The first is a ballistics code, used to determine the kinetic energy $E$ of a ball when it hits the ground, with a path depending on its initial height $y_{0}$, initial velocity $v_{0}$, mass $m$ and initial angle $\theta_{0}$. We'll assume we're near the Earth's surface and aside from the initial launch height, we're launching over a flat surface. This can be represented as a functional $E\left(y_{0}, v_{0}, m, \theta_{0}\right)$.

The second physics model is an impact code that estimates the diameter of the crater $D$ made when a ball impacts. With a few simplifying assumptions ( $D$ is under $1 \mathrm{~km}$, the impact is near Earth's surface, the impact is in dry sand with fixed density), size $D$ can be determined as a function of the ball's kinetic energy when impacting $E$, its mass $m$, and its radius $r: D(E, m, r)$.

We can see that the input $E$ for the impact model is calculated by the ballistics code. In RAVEN the EnsembleModel will automatically detect this, and run the ballistics code before the impact code in each iteration.

In general, the EnsembleModel also has some finite capacity for resolving circular dependen- 
cies using Picard iteration. See the manual for more information on this capability.

Setting up an EnsembleModel can be more complex than other models, so we'll walk through the input using our example outlined above. For our purposes, we're going to let RAVEN perturb the codes using the GenericCode interface. We'll call the ballistics code ballistics.py with keyword input file ballistics_input.txt and similarly the impact code impact.py with keyword input file impact_input.txt.

The RAVEN input file for this example is located at

raven/tests/framework/user_guide/EnsembleModel/basic.xml

with run directory run_basic. The models and inputs are in the run directory.

Now we turn our attention to the RAVEN input file. We will discuss <DataObjects>, $<$ Files $>$, <Models>, and <Steps $>$ in detail; the rest are typical usage and need no special attention.

\subsubsection{DataObjects}

The key to a successful EnsembleModel is setting up the DataObject inputs and outputs. Each model has a <TargetEvaluation> DataObject that specifies what the inputs and outputs are for that model. In addition, any <Output > DataObjects in the <MultiRun > step can collect any or all of the variables used throughout the EnsembleModel calculation, either as inputs or outputs.

In this example, our convention is to name the <TargetEvaluation $>$ DataObjects as 'model_data', replacing 'model' with the model name. Additionally, we add a DataObject to store the final results of the <MultiRun> step:

raven/tests/framework/user_guide/EnsembleModel/basic.xml

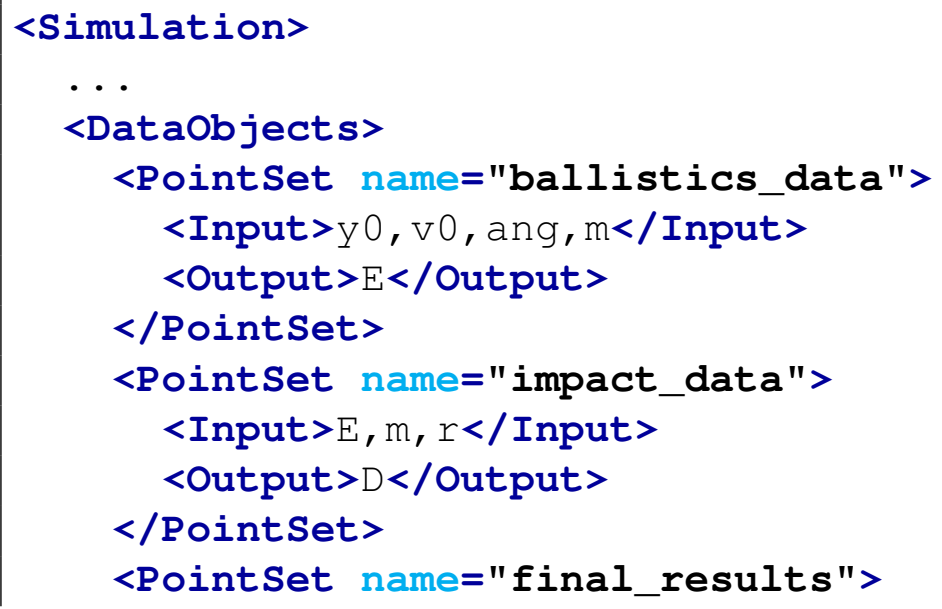




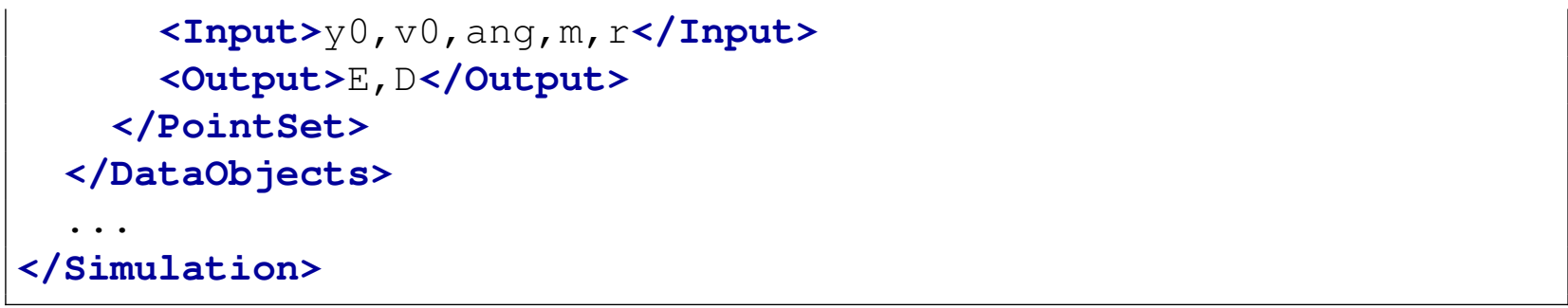

In this example, we see three DataObjects. 'ballistics_data' determines the inputs and outputs of the ballistics.py model, so for its input space we have y0, v0, ang, m, which correspond to $y_{0}, v_{0}, \theta_{0}, m$; for the output, we have $\mathrm{E}$ (for $E$ ).

The second DataObject, ' impact_data', delineates the inputs and outputs of the impact . py model, so for its input space we have $E, m, r$, while in the output space we have D. RAVEN will use these first two DataObjects to map the order in which these two models should be run (first ballistics, then impact) and transfer data from one to the next.

The third DataObject, ' final_results', can contain any information we want it to. In this case, we want to collect all the variables in their original spaces, so we take $y 0, v 0, a n g, m, r$ as inputs and $\mathrm{E}, \mathrm{D}$ as outputs.

\subsubsection{Files}

Since we know both of our models take input files to set the variable values, we inform RAVEN about the template input files and give those files RAVEN names in the $\langle$ Files $\rangle$ block. In this example, our input file for ballistics.py is ballistics_template.txt, which we simply name 'ballistics_input'. Similarly, for impact.py the input file is impact_template.txt, which we name' impact_input':

raven/tests/framework/user_guide/EnsembleModel/basic.xml

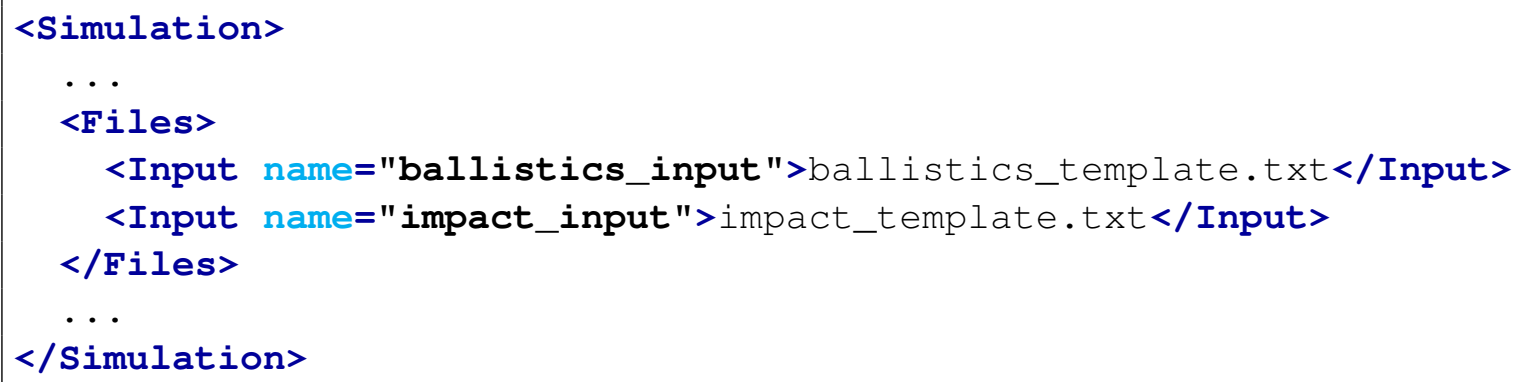

We will pause to note that the file ballistics_template.txt is different than the file ballistics_input.py, and similarly for the impact code. In the template file, we've replaced variable values with the $\$ R A V E N-\$$ wildcard, since we will be using the Generic Code model for these two models. Having a template input file may not be required for all code interfaces, but it is 
required for the Generic interface. See more about this interface in the Models section and in the user manual.

\subsubsection{Models}

Once we've specified the DataObjects and files, we are prepared to set up the $<$ Models $>$ block. To set up an EnsembleModel, we define each sub-module by itself as if it were a stand-alone RAVEN model, and then combine them by defining an <EnsembleModel $>$ :

raven/tests/framework/user_guide/EnsembleModel/basic.xml

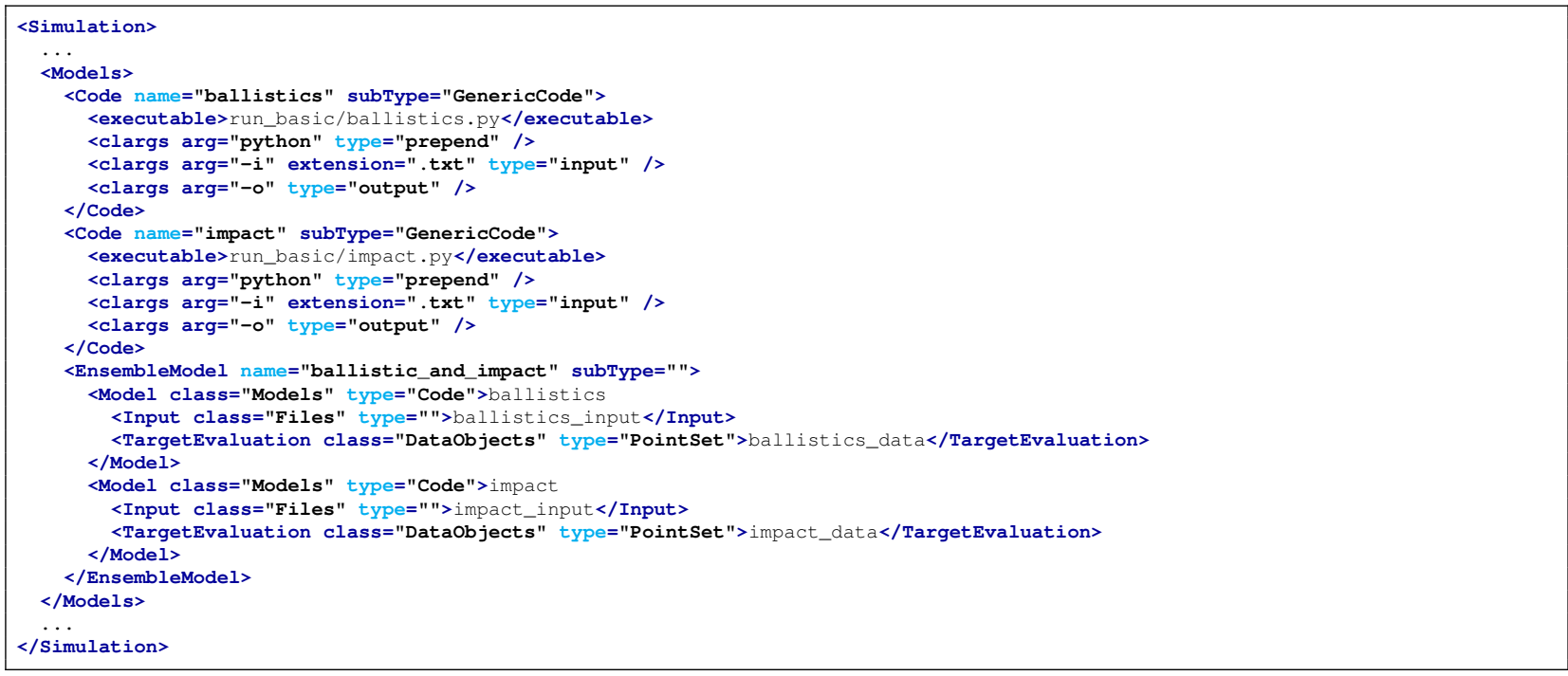

The first model we will look at is the definition of the 'ballistics' model. We note that is uses the 'GenericCode' subType, so it will use the interface as described in the user manual, where wildcards in the input file are replaced with RAVEN's sampled values. The <clargs> (command line arguments) include the 'python' command, the ' $-i$ ' flag to specify the input file, and the ' $-0^{\prime}$ flag to specify the output file. These nodes are specific to the Generic code interface and will not apply to all codes or models. The ' Impact' model is defined in a similar manner.

With both Models defined, we can construct the <EnsembleModel>, which we've named 'ballistic_andimpact' to describe the codes that are paired (you can name this whatever you want). There is no subType for the <EnsembleModel $>$ currently, so we leave it blank.

Within the $<$ EnsembleModel $>$ node there are two $<$ Model $>$ nodes listed, which will inform the EnsembleModel which models need coupling. The order listed does not matter; the EnsembleModel will sort out the order based on the <TargetEvaluation> DataObject of each model.

The first $<$ Model $>$ we list within the $<$ EnsembleModel $>$ is the ballistics model. Note 
that the class and type are used along with the name to find the right model. Note also the name of the model goes in the text of the <Model> node, right after the first closing angle bracket. The subnodes of the 'ballistics' model are the <Input>, which points to the input template input file we defined in the $\langle$ Files $>$ block; and the $\langle$ TargetEvaluation $>$, which points to the <PointSet $>$ we defined in the <DataObjects $>$ block. These are critical to allowing the EnsembleModel both to run the 'ballistics' model correctly, as well as the entire ensemble in a sensible manner.

In general, some models (such as a $<$ ROM $>$ or $<$ ExternalModel $>$ ) do not require any files as inputs. In this case, the <Input> for an input-less model should be a placeholder DataObject, usually with the same input space as the $<$ TargetEvaluation $>$ DataObject, and a placeholder output. For more information, see examples running ExternalModels in these guides as well as the user manual.

After the 'ballistics' model, we see a similar structure pointing to the 'impact' model. The only differences between this model specification and the ballistics model specificatoin are the names of the <Input > file and <TargetEvaluation> DataObject.

With the two models defined, and the EnsembleModel assembled, no more work is required to prepare these two models to run. More complicated systems may require additional nodes in the EnsembleModel; see the user manual for details.

\subsubsection{Steps}

Finally, to put all the pieces together, we consider the <Steps > node:

raven/tests/framework/user_guide/EnsembleModel/basic.xml

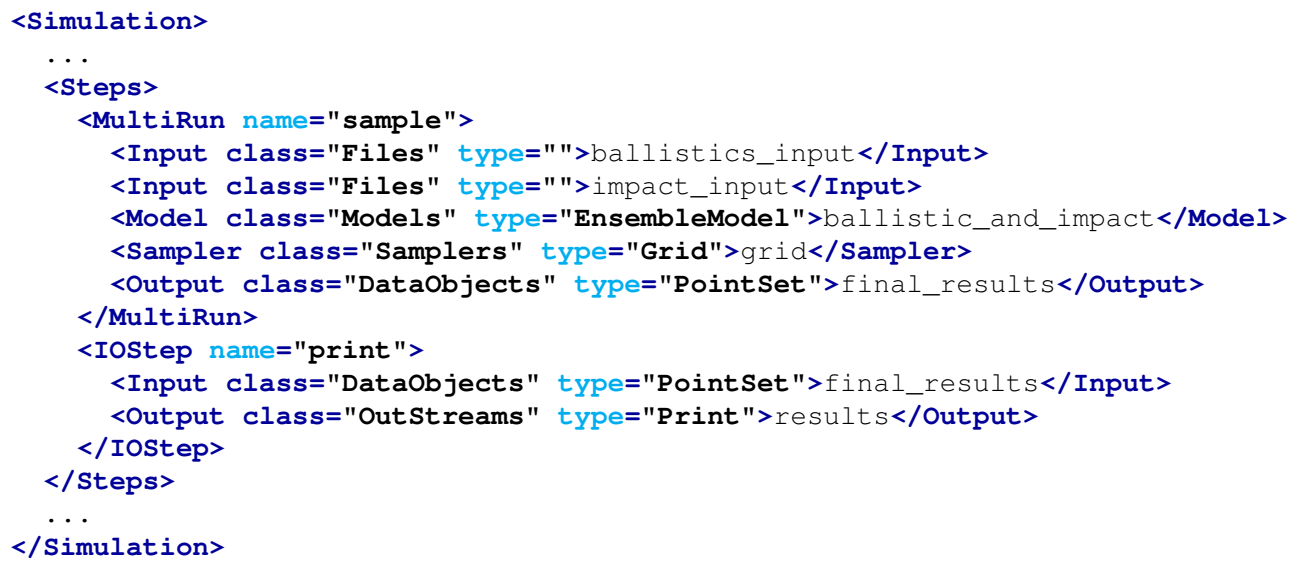

We take two steps in this simulation, one <MultiRun>called ' sample' to sample the EnsembleModel on a grid, and one <IOStep> to print the results. These are constructed just as they would be with any other model; as far as the <Steps $>$ are concerned, the EnsembleModel is like 
any other model. Note that we include the two template input files as <Input> nodes for the sampling step.

We do not discuss the <Distributions>, <Samplers>, or <OutStreams > here. These operate in general as they would in any other RAVEN input file. We will note, however, that the <Grid> sampler named ' grid' samples all the inputs that are needed by either of the submodels, unless the inputs are provided by another model. In this case, that means we need to sample $y_{0}, \theta_{0}, v_{0}, m, r$ but we do not sample $E$ since it is calculated by the 'ballistics' model.

We can find all the sampled and calculated values from both of the models in the output produced by this run, found at

raven/tests/framework/user_guide/EnsembleModel/run_basic/results.csv

\subsection{ExternalModel in EnsembleModel}

There are a few details that can make handling <ExternalModel> models challenging in EnsembleModel. We'll cover a few of these to help smooth over some potential bumps.

\subsubsection{Input Placeholder DataObject}

Firstly, as noted above, both ExternalModel and ROM differ from Code models in a significant way: they (usually) do not require any input files. So what do you put as the <Input $>$ for an ExternalModel or ROM? To maintain consistency, we leave the <Input> node in place. Instead of specifying a file however, a placeholder DataObject is used instead. A placeholder DataObject has variables listed in its input, but for output either the <Output> node is omitted, or the special keyword OutputPlaceHolder is used instead. For example, see the excerpt from a regression test:

raven/tests/framework/ensembleModelTests/index_input_output.xml

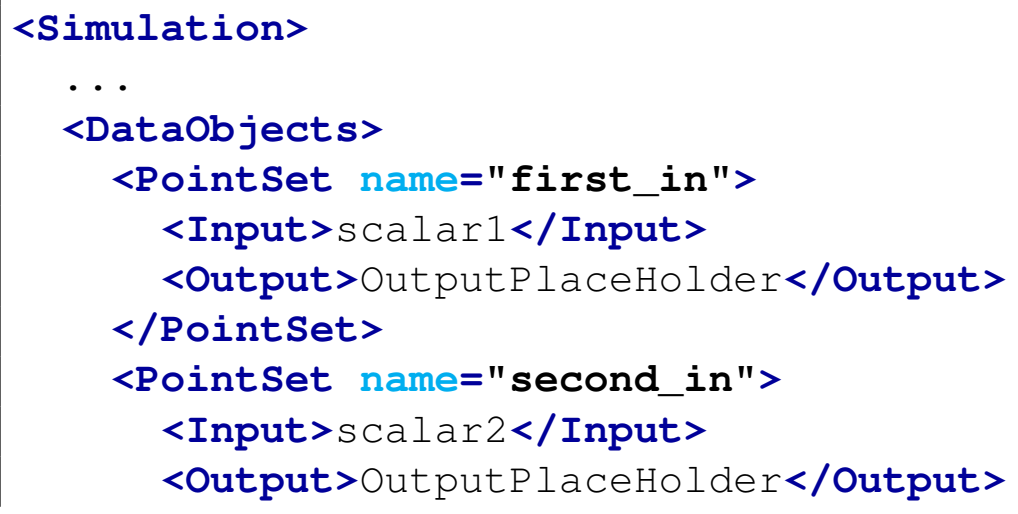




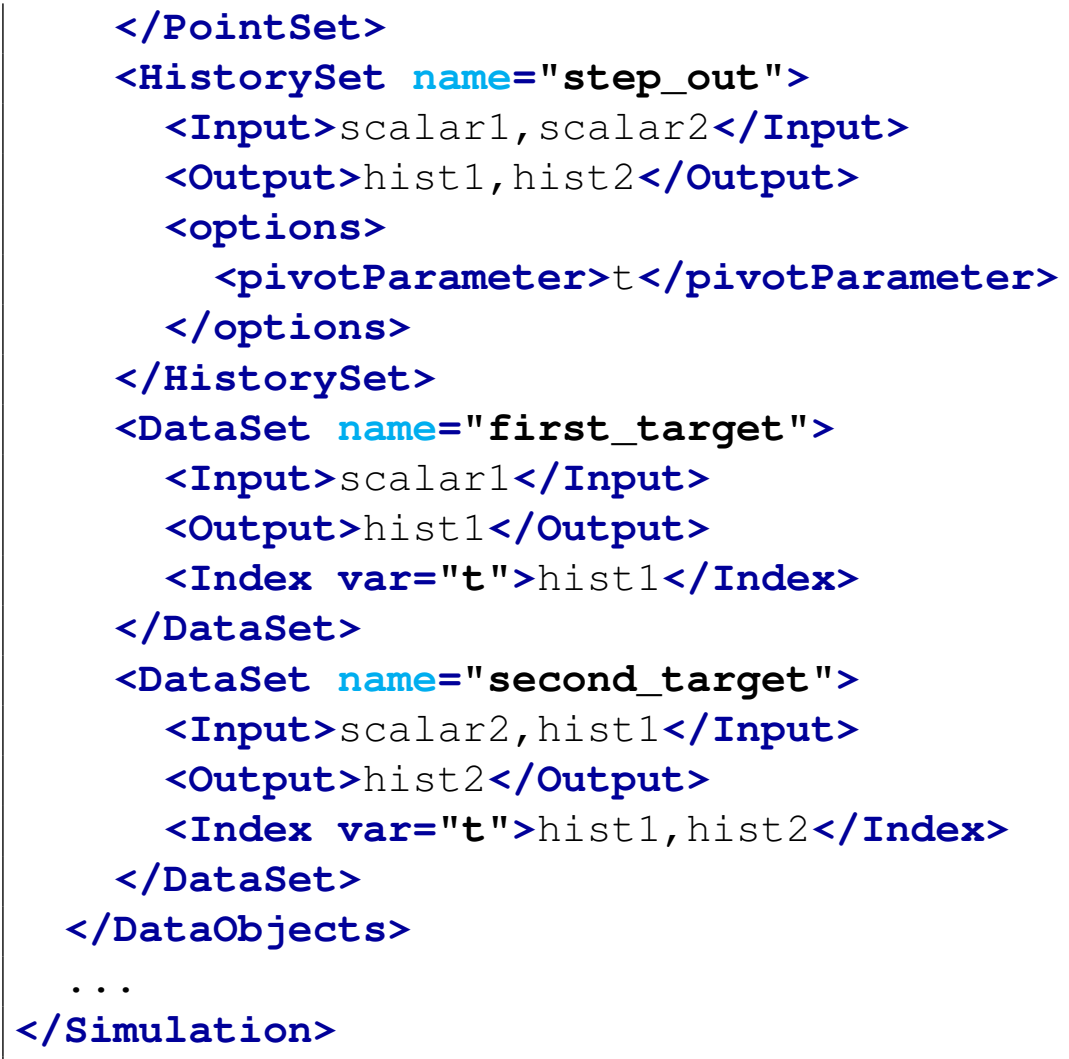

The inputs 'first_in' and 'second_in' are placeholder DataObject, with a scalar input variable for the <Input> and placeholder OutputPlaceHolder as <Output>. It is always a $<$ PointSet $>$ type of DataObject. The key OutputPlaceHolder is recognized specially in RAVEN and translates into an empty output space. This type of DataObject is the only suitable input for an ExternalModel or ROM without an input file.

\subsubsection{DataSet}

Because of the complexity of EnsembleModel, it is possible to have mixed scalars and vectors as variable inputs to a particular model in the Ensemble. Furthermore, it is possible to have multiple vector variables that depend on different independent variables (for example, "time" and "space"). To accommodate this, the <DataSet $>$ DataObject can be used. More can be found on this data structure in the RAVEN user manual.

\subsubsection{Scalar Variables}

Because of the flexibility of the EnsembleModel, and to maintain a level of consistency across all variables, scalar variables in the EnsembleModel are stored as Numpy arrays with length 1. In 
general this should be transparent for most operations; however, occasionally it may be required to access a scalar variable by accessing it at index 0 . For example, see the variable "scalar2" in a model used in the regression tests for the EnsembleModel:

raven/tests/framework/ensembleModel Tests/IndexInputOutput/model2.py

import numpy as np

def $\operatorname{run}(o b j, d c t)$ :

obj.t \#just making sure it's available here

obj.hist 2 =obj.hist1 +obj.scalar2[0]

\subsubsection{Independent Variables}

One feature of ExternalModel is their capacity to take variables exactly as they are from RAVEN, without going through file IO. As a result, EnsembleModel frequently contain models that produce pivot-dependent data that can be used as inputs for other models in the Ensemble. Several terms aptly describe these variables on which other variables depend, including "independent variables," "indexes", and "pivots". In general, we refer to them as "indexes". While the index is often "time" or something similar, it can be any monotonically-increasing variable.

However, when it is stored in RAVEN, the indexes are not stored like other variables, because it is a coordinate axes that supports other variables. This allows RAVEN to do a great many flexible operations internally. However, it also means that any time you want to pass an index variable from one model to an ExternalModel, it can only come attached to another variable that depends on that input.

For example, consider two models. In the first model, a path followed by a charged particle is traced out in time. The outputs of the first model are time $t$, lateral position $x$, and vertical position $y$. The second model calculates the energy of the particle at each point in time; however, the second model is written such that it does not require any specific time values (it just reads them from the input).

To run these two models in Ensemble configuration, <TargetEvaluation> DataObject of the second model must request one of the time-dependent variables from the first model, $x$ or $y$. If $t$ is directly requested, it will not be made available in the second ExternalModel. Additionally, in the <Model> definition, all variables (dependent or index) should be listed. In this example, when defining the model, $t$ should be listed as well as $x$ in the $\langle$ variables $>$ node in the second $<$ Model $>$ definition.

For an example of time dependent data passing, consider the regression test example at 
raven/tests/framework/ensembleModelTests/index_intput_output.xml 


\section{Appendices}

\section{A Document Version Information}

tag_number_23-192-gb3087a024

62a386fc1da99a1d77afb73e2ad19e7e47fd5a72 Paul Talbot

Tue, 22 May 2018 12:47:08 -0600 


\section{References}

[1] "Neams: The nuclear energy advanced modeling and simulation program," Tech. Rep. ANL/NE-13/5.

[2] "Light water reactor sustainability program integrated program plan," Tech. Rep. INL-EXT11-23452, April 2013.

[3] R.-D. development team, "Relap5/mod3.3 code manual," tech. rep., Idaho National Laboratory, October 2015.

[4] C. Rabiti, A. Alfonsi, J. Cogliati, D. Mandelli, R. Kinoshita, and S. Sen, "Raven user manual," tech. rep., Idaho National Laboratory, 2015.

[5] A. Alfonsi, C. Rabiti, D. Mandelli, J. Cogliati, and B. Kinoshita, "Raven as a tool for dynamic probabilistic risk assessment: Software overview," in Proceedings of International Conference of mathematics and Computational Methods Applied to Nuclear Science and Engineering (M\&C 2013), Sun Valley (Idaho), pp. 1247-1261, 2013.

[6] A. Alfonsi, C. Rabiti, D. Mandelli, J. Cogliati, B. Kinoshita, and A. Naviglio, "Dynamic event tree analysis through raven," in Proceedings of ANS PSA 2013 International Topical Meeting on Probabilistic Safety Assessment and Analysis, Columbia (South Carolina), 2013.

[7] C. Rabiti, A. Alfonsi, D. Mandelli, J. Cogliati, and R. Kinoshita, "Deployment and overview of raven capabilities for a probabilistic risk assessment demo for a pwr station blackout," Tech. Rep. INL/EXT-13-29510, Idaho National Laboratory (INL), 2013.

[8] A. Alfonsi, C. Rabiti, D. Mandelli, J. Cogliati, and B. Kinoshita, "Raven and dynamic probabilistic risk assessment: Software overview," in Proceedings of ESREL European Safety and Reliability Conference (ESREL 2014), Wrocklaw (Poland), 2014.

[9] D. Anders, R. Berry, D. Gaston, R. Martineau, J. Peterson, H. Zhang, H. Zhao, and L. Zou, "Relap-7 level 2 milestone report: Demonstration of a steady state single phase pwr simulation with relap-7,” Tech. Rep. INL/EXT-12-25924, Idaho National Laboratory (INL), 2012. 


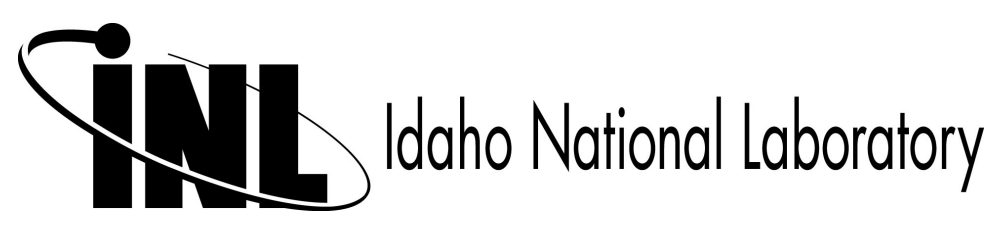

